\title{
Ligand-Promoted, Boron-Mediated Chemoselective Carboxylic Acid Aldol Reaction
}

\author{
Hideoki Nagai, ${ }^{1}$ Yuya Morita, ${ }^{1}$ Yohei Shimizu ${ }^{*}, 1$ \& Motomu Kanai ${ }^{*, 1,2}$ \\ ${ }^{1}$ Graduate School of Pharmaceutical Sciences, The University of Tokyo, 7-3-1 Hongo, Bunkyo-ku, \\ Tokyo 113-0033, Japan \\ ${ }^{2}$ ERATO, Japan Science and Technology Agency (JST), Kanai Life Science Catalysis Project, 7-3-1 \\ Hongo, Bunkyo-ku, Tokyo 113-0033, Japan \\ *e-mail: y-shimizu@mol.f.u-tokyo.ac.jp; kanai@mol.f.u-tokyo.ac.jp
}

\section{Table of Contents}

1. General Method (S2)

2. Preliminary Investigation for Aldol Reaction of Carboxylic Acids (S3-S4)

3. General Procedure (S5)

4. ${ }^{11}$ B NMR experiments (S6-S7)

5. Epimerization Check (S8)

6. Synthesis and Characterization of Known/New Compounds (S9-S31)

7. NMR Charts (S32-S127) 


\section{General Method}

${ }^{1} \mathrm{H},{ }^{13} \mathrm{C},{ }^{19} \mathrm{~F}$ and ${ }^{11} \mathrm{~B}$ NMR spectra were recorded on JEOL ECX500 $\left(500 \mathrm{MHz}\right.$ for ${ }^{1} \mathrm{H}$ NMR and $125 \mathrm{MHz}$ for ${ }^{13} \mathrm{C}$ NMR), and JEOL ECS400 (400 MHz for ${ }^{1} \mathrm{H}$ NMR, $100 \mathrm{MHz}$ for ${ }^{13} \mathrm{C}$ NMR, 368 $\mathrm{MHz}$ for ${ }^{19} \mathrm{~F}$ NMR and $125 \mathrm{MHz}$ for ${ }^{11} \mathrm{~B}$ NMR) spectrometer. Chemical shifts were reported in the scale relative to the solvent used as an internal reference for ${ }^{1} \mathrm{H}\left(\delta=7.26 \mathrm{ppm}\right.$ for $\mathrm{CDCl}_{3}, 2.05 \mathrm{ppm}$ for acetone- $d_{6}$ ) and ${ }^{13} \mathrm{C}$ NMR $\left(\delta=77.00 \mathrm{ppm}\right.$ for $\mathrm{CDCl}_{3}, 206.26 \mathrm{ppm}$ for acetone- $d_{6}$ ). Fluorine chemical shifts are reported relative to $\alpha, \alpha, \alpha$-trifluorotoluene $(\delta=-63.44 \mathrm{ppm})$ as an external reference. Boron chemical shifts are reported relative to $\mathrm{BF}_{3} \cdot \mathrm{Et}_{2} \mathrm{O}(\delta=0 \mathrm{ppm})$ as an external reference. Infrared (IR) spectra were recorded on a JASCO FT/IR 410 Fourier transform infrared spectrophotometer. ESI-mass spectra were measured on a Waters ZQ4000 spectrometer, a SHIMADZU LCMS-2020 (for LRMS), and a JEOL JMS-T100LC AccuTOF spectrometer (for HRMS). The enantiomeric excesses (ee's) were determined by HPLC analysis conducted by JASCO HPLC systems (pump: PU-2080; detector: UV-2075, measured at $254 \mathrm{~nm}$; chiral column; mobile phase: hexane/2-propanol). Optical rotations were measured on a JASCO P-1010 polarimeter. Column chromatographies were performed with silica gel Merck 60 (230-400 mesh ASTM) or neutral silica gel $60 \mathrm{~N}$ (KANTO CHEMICAL, spherical, neutral, 40-100 $\mu \mathrm{m}$ ). Preparative recycling gel permeation chromatography (GPC) was performed with JAI LC-9210NEXT instrument equipped with JAIGEL-1H and JAIGEL-2H columns using $\mathrm{CHCl}_{3}$ as an eluent. All non-commercially available compounds were prepared and characterized as described in Section 6 of this SI. DBU was purified by distillation from $\mathrm{CaH}_{2}$ and used under argon atmosphere. Reagents that were not further described were purchased from Aldrich, Tokyo Chemical Industry Co., Ltd. (TCI), Kanto Chemical Co., Inc., and Wako Pure Chemical Industries, Ltd. and used without further purification. 


\section{Preliminary Investigation for Aldol Reaction of Carboxylic Acids}

Preliminary investigation was conducted with propionic acid (2a) and benzaldehyde (1a) (Table S1). Mild organic bases such as DBU (entry 1), $\mathrm{Et}_{3} \mathrm{~N}$, and ${ }^{i} \mathrm{Pr}_{2} \mathrm{NEt}$ (data not shown) were unable to produce the targeted aldol adduct 3aa in the absence of a boron compound. We subsequently examined the impact of several boron compounds (1 equiv) on this reaction. Lewis acidic $\mathrm{BF}_{3} \cdot \mathrm{Et}_{2} \mathrm{O}$, $\mathrm{BBr}_{3}$ or $\mathrm{B}(\mathrm{OMe})_{3}$ did not afford 3aa (entries 2-4). Boric acid, phenylboronic acid, 9-BBN or pinacol borane (PinBH) also did not give 3aa (entries 5-8), while catechol borane (CatBH) afforded the aldol adduct 3aa in $15 \%$ yield (entry 9). Ultimately, $\mathrm{BH}_{3} \cdot \mathrm{SMe}_{2}$ displayed the best performance, furnishing the desired aldol adduct 3aa in 30\% yield and high syn-selectivity (syn/anti $=8.5 / 1$; entry 10 ). Further investigation is described in the manuscript (Table 1).

Table S1. Investigation of boron sources.

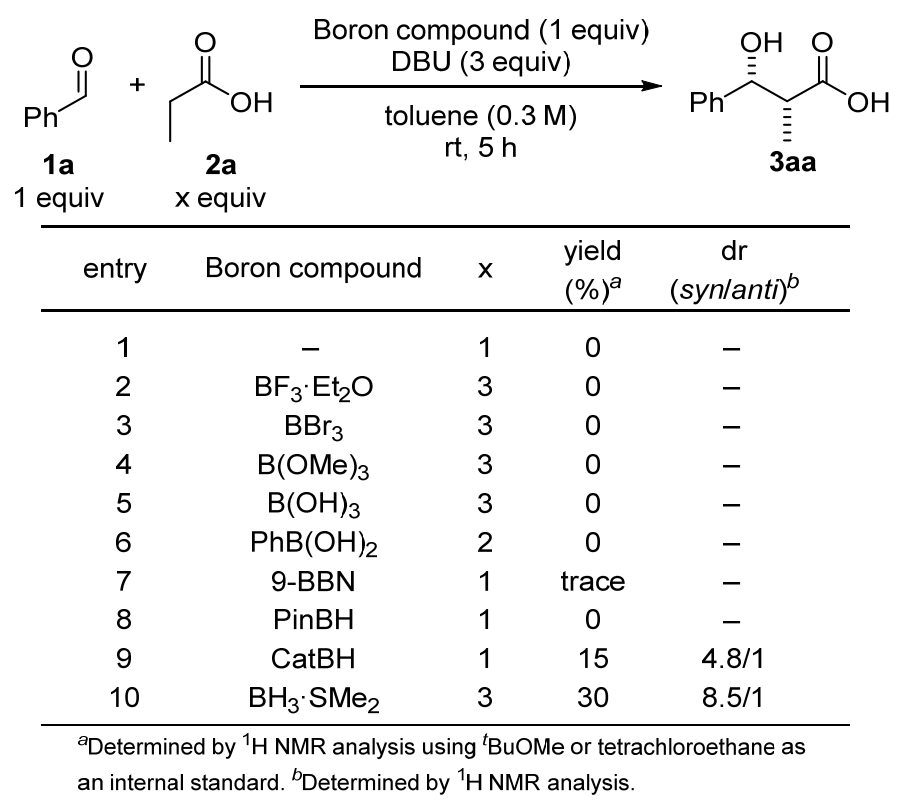

To improve the reactivity of the aldol reaction, we examined organic and inorganic bases (Table S2). Tertiary amines, ${ }^{i} \mathrm{Pr}_{2} \mathrm{NEt}$ and $\mathrm{NEt}_{3}$, promoted the reaction, albeit in lower reactivity and diastereoselectivity than DBU (entries 2 and 3). On the other hand, less basic DABCO and pyridine were totally ineffective (entries 4 and 5). Carbonate bases were examined as inorganic bases, but the desired product 3aa was not obtained at all (entries 6 and 7). Based on this screening, we selected DBU as the optimum base for the carboxylic acid aldol reaction. 
Table S2. Investigation of bases.

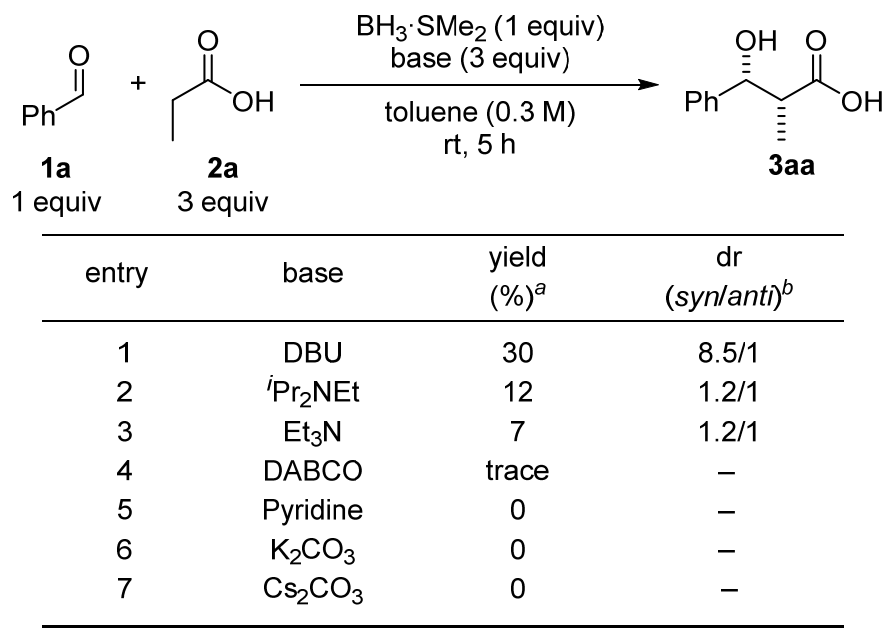

${ }^{a}$ Determined by ${ }^{1} \mathrm{H}$ NMR analysis using ${ }^{t} \mathrm{BuOMe}$ or tetrachloroethane as an internal standard. ${ }^{b}$ Determined by ${ }^{1} \mathrm{H} \mathrm{NMR}$ analysis. 


\section{General Procedure}

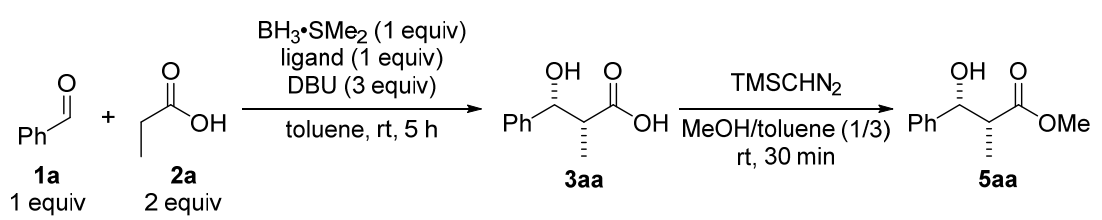

A flame-dried $20 \mathrm{~mL}$ test tube, equipped with a magnetic stirring bar, was charged with Ts-L-Leu $(86 \mathrm{mg}, 0.30 \mathrm{mmol})$ and anhydrous toluene $(1.0 \mathrm{~mL})$, before $\mathrm{BH}_{3} \cdot \mathrm{SMe}_{2}(1.0 \mathrm{M}$ in DCM, $300 \mu \mathrm{L}, 0.30 \mathrm{mmol}$ ) was added. After stirring for $30 \mathrm{~min}$, propionic acid (2a) $(45 \mu \mathrm{L}, 0.60 \mathrm{mmol})$ was added. After stirring for $30 \mathrm{~min}, \mathrm{DBU}(135 \mu \mathrm{L}, 0.90 \mathrm{mmol})$ and benzaldehyde (1a) $(31 \mu \mathrm{L}, 0.30$ mmol) were added sequentially, and the reaction mixture was stirred for $5 \mathrm{~h}$ at room temperature under an argon atmosphere. Subsequently, the reaction was quenched with aq. $\mathrm{HCl}(1.0 \mathrm{M})$ and the organic layer was separated. The aqueous layer was extracted with EtOAc. The combined organic layer was washed with aq. $\mathrm{HCl}(1.0 \mathrm{M})$, dried over $\mathrm{Na}_{2} \mathrm{SO}_{4}$, filtered and concentrated under reduced pressure to afford the crude product 3aa.

Subsequent methyl esterification was carried out without further purification. To a solution of

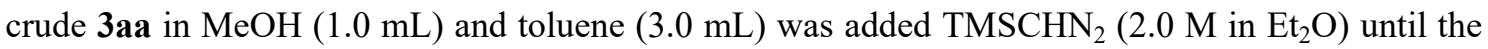
color of the solution became yellow. After stirring for $30 \mathrm{~min}$ at room temperature, a few drops of acetic acid were added until the yellow color of the solution disappeared. The solvent was evaporated under reduced pressure and the remaining residue was purified by silica gel column chromatography $($ EtOAc/hexane $=1 / 9)$, affording $\mathbf{5 a a}$ as a colorless oil $(46 \mathrm{mg}, 79 \%$ yield, syn/anti $=>20 / 1)$.

syn-Methyl 3-hydroxy-2-methyl-3-phenylpropanoate (5aa: methyl ester of 3aa)

A colorless oil; Yield: 79\%; syn/anti $=>20 / 1 ; \mathrm{R}_{\mathrm{f}}=0.33($ EtOAc/hexane $=1 / 4) ;{ }^{1} \mathrm{H}$ NMR $(500 \mathrm{MHz}$, $\left.\mathrm{CDCl}_{3}\right): \delta 1.05(\mathrm{~d}, J=6.9 \mathrm{~Hz}, 3 \mathrm{H}), 2.69-2.74(\mathrm{~m}, 1 \mathrm{H}), 3.60(\mathrm{~s}, 3 \mathrm{H}), 5.04(\mathrm{~d}, J=4.0 \mathrm{~Hz}, 1 \mathrm{H})$, 7.18-7.27 (m, 5H); ${ }^{13} \mathrm{C}$ NMR $\left(125 \mathrm{MHz}, \mathrm{CDCl}_{3}\right): \delta 10.7,46.3,51.9,73.6,125.9,127.5,128.3,141.3$, 176.2; IR (neat): 3482, 2951, 1733, 1455, 1199, 1035, $770 \mathrm{~cm}^{-1}$; LRMS (ESI): m/z 217 [M+Na $]^{+}$; HRMS (ESI): $\mathrm{m} / \mathrm{z}$ calcd for $\mathrm{C}_{11} \mathrm{H}_{14} \mathrm{O}_{3}[\mathrm{M}+\mathrm{Na}]^{+} 217.0841$ Found 217.0851 .

The spectral data of syn-5aa was identical with the reported values of the syn-isomer ${ }^{1}$. HPLC (chiral column: CHIRALCEL OD; solvent: hexane/2-propanol = 100/3; flow rate: $1.0 \mathrm{~mL} / \mathrm{min}$; detection: at $254 \mathrm{~nm} ; \mathrm{rt}$ ): $t_{\mathrm{R}}=12.4 \mathrm{~min}$ (major) and $15.1 \mathrm{~min}$ (minor) for $23 \%$ ee. Based on the comparison of the reported retention time for the enantiomers, the absolute configuration of syn-5aa was determined to be $(2 R, 3 R)$.

The major isomers of other products were also assigned to be syn by analogy.

${ }^{1}$ Yamashita, Y.; Ishitani, H.; Shimizu, H.; Kobayashi, S. J. Am. Chem. Soc. 2002, 124, 3292. 


\section{4. ${ }^{11}$ B NMR Experiments}

The following ${ }^{11} \mathrm{~B}$ NMR experiments in $\mathrm{C}_{6} \mathrm{D}_{6}$ were carried out to obtain mechanistic insight into the role of two equivalents of carboxylic acid. ${ }^{11} \mathrm{~B}$ NMR spectra after complexation of $\mathrm{BH}_{3} \cdot \mathrm{SMe}_{2}$ (neat, $17 \mu \mathrm{L}, 0.18 \mathrm{mmol}$ ), Ts-L-Val $(49 \mathrm{mg}, 0.18 \mathrm{mmol}$ ) and 1 equiv of propionic acid (2a) $(14 \mu \mathrm{L}, 0.18 \mathrm{mmol})$ in $\mathrm{C}_{6} \mathrm{D}_{6}(600 \mu \mathrm{L})$ showed two major species $(5.5,2.7 \mathrm{ppm})$ (Fig. S1), indicating insufficient formation of active precursor for enolization by DBU. In contrast, complexation with 2 equiv of propionic acid (2a) $(28 \mu \mathrm{L}, 0.36 \mathrm{mmol})$ resulted in predominant formation of one major intermediate $(2.3 \mathrm{ppm})$ (Fig. S2). The results suggest that the second equivalent of carboxylic acid facilitates the formation of active precursor for enolization, thus enhances the reactivity. The structure of the active precursor is tentatively assigned as the cyclic intermediate $\mathbf{A}$, similar to the previously reported complexes ${ }^{2}$.

The ${ }^{11} \mathrm{~B}$ NMR was also measured after the aldol reaction using propionic acid (2a) $(45 \mu \mathrm{L}, 0.60$ mmol), benzaldehyde (1a) $(31 \mu \mathrm{L}, 0.30 \mathrm{mmol}$ ), Ts-L-Val (81 mg, $0.3 \mathrm{mmol}), \mathrm{BH}_{3} \cdot \mathrm{SMe}_{2}$ (neat, $29 \mu \mathrm{L}$, $0.30 \mathrm{mmol})$, and DBU $(135 \mu \mathrm{L}, 0.90 \mathrm{mmol})$ in $\mathrm{C}_{6} \mathrm{D}_{6}(1 \mathrm{~mL})$. The ${ }^{11} \mathrm{~B}$ NMR spectra indicated one major singlet peak at $3.5 \mathrm{ppm}$ (Fig. S3), which is assigned to the cyclic borate compound $\mathbf{C}$.

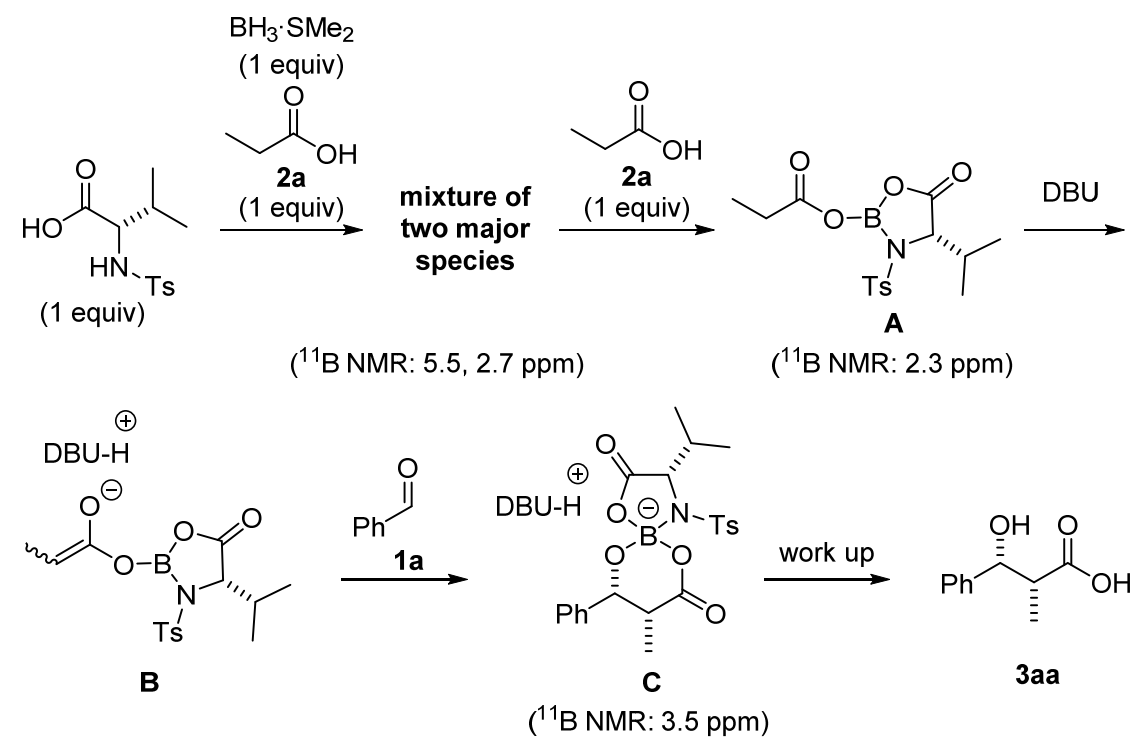

2 (a) Kiyooka, S.; Kaneko, Y.; Komura, M.; Matsuo, H.; Nakano, M. J. Org. Chem. 1991, 56, 2276. (b) Yeung, Y.-Y.; Corey, E. J. Org. Lett. 2008, 10, 3877. 
Figure $\mathbf{S 1} \cdot \mathrm{BH}_{3} \cdot \mathrm{SMe}_{2}$ (1 equiv) + Ts-L-Val (1 equiv) + propionic acid (2a) (1 equiv)
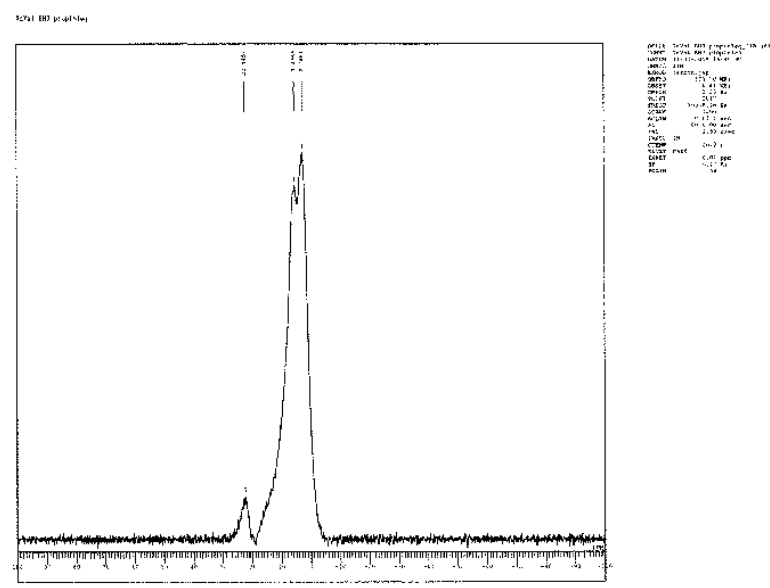

Figure $\mathbf{S 2} \cdot \mathrm{BH}_{3} \cdot \mathrm{SMe}_{2}$ (1 equiv) + Ts-L-Val (1 equiv) + propionic acid (2a) (2 equiv)
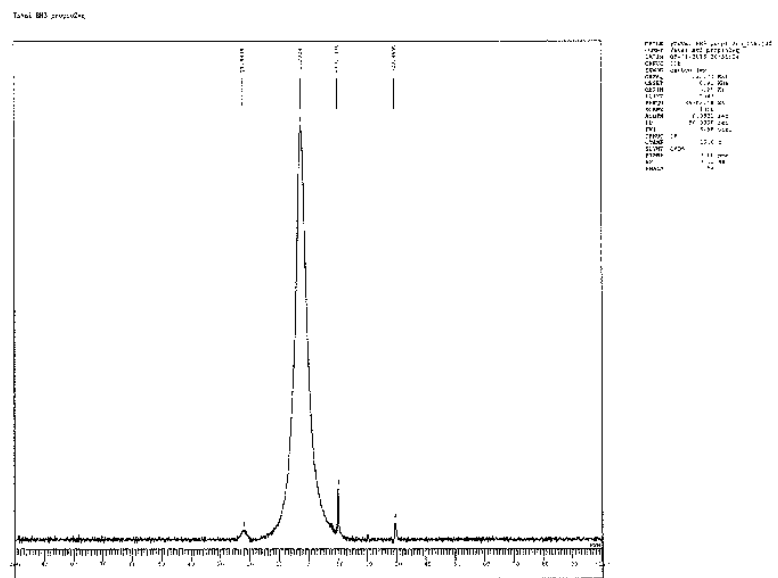

Figure S3. ${ }^{11}$ B NMR of intermediate $\mathbf{C}$
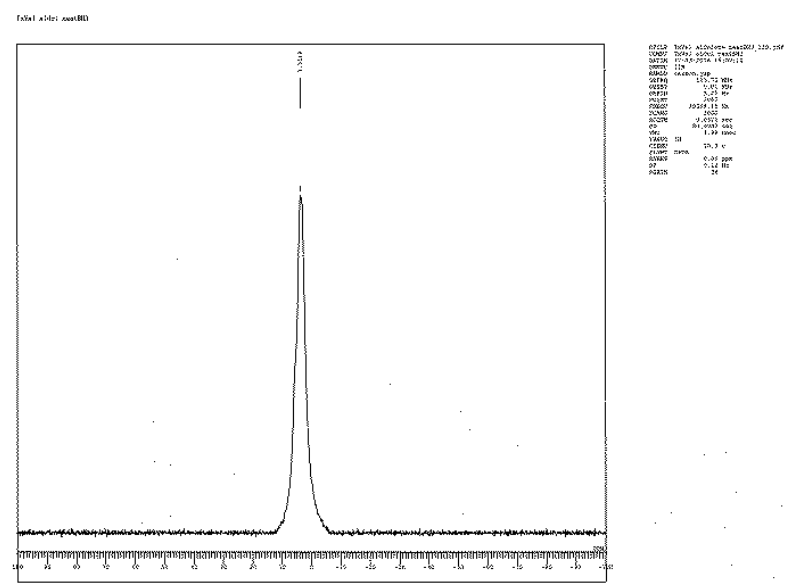


\section{Epimerization Check}

A flame-dried $20 \mathrm{~mL}$ test tube, equipped with a magnetic stirring bar, was charged with Ts-L-Leu (94 mg, $0.33 \mathrm{mmol})$ and anhydrous toluene $(1.1 \mathrm{~mL})$, before $\mathrm{BH}_{3} \cdot \mathrm{SMe}_{2}(1.0 \mathrm{M}$ in DCM, $330 \mu \mathrm{L}, 0.33 \mathrm{mmol})$ was added. After stirring for $30 \mathrm{~min}$, propionic acid (2a) $(25 \mu \mathrm{L}, 0.33 \mathrm{mmol})$ and a solution of $3 \mathrm{aa}(60 \mathrm{mg}, 0.33 \mathrm{mmol}$, syn/anti $=1 / 1)$ in toluene $(500 \mu \mathrm{L})$ was added. After stirring for $30 \mathrm{~min}$, DBU ( $148 \mu \mathrm{L}, 0.99 \mathrm{mmol})$ was added, and the reaction mixture was stirred for 5 $\mathrm{h}$ at room temperature under an argon atmosphere. Subsequently, the reaction was quenched with aq. $\mathrm{HCl}(1.0 \mathrm{M})$ and the organic layer was separated. The aqueous layer was extracted with EtOAc. The combined organic layer was washed with aq. $\mathrm{HCl}(1.0 \mathrm{M})$, dried over $\mathrm{Na}_{2} \mathrm{SO}_{4}$, filtered and concentrated under reduced pressure to recover the crude product 3aa $(91 \%$ NMR yield, syn/anti $=$ $1.1 / 1)$.

The result strongly indicates that kinetically controlled products are generated in this reaction. 


\section{Synthesis and Characterization of Known/New Compounds}

\section{6-1. Synthesis and Characterization of Aldehydes}

Benzaldehyde (1a) was purified by distillation from $\mathrm{CaH}_{2}$. Hydrocinnamaldehyde (1b) was purified by distillation from $\mathrm{MgSO}_{4} \cdot \mathbf{1 h}^{3}$ was prepared by following the same procedure as described in the literature. 1i was prepared by following the procedures shown below. Other aldehydes were purchased and used without further purification.

\section{Preparation of Aldehyde}

(1i)

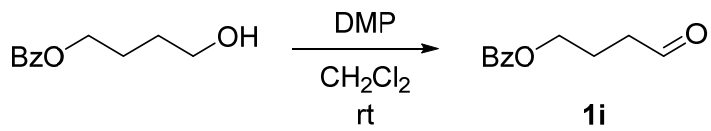

Following the reported procedure, 4-hydroxybutyl benzoate was prepared ${ }^{4}$. To a solution of 4-hydroxybutyl benzoate $(722 \mathrm{mg}, 3.7 \mathrm{mmol})$ in $\mathrm{CH}_{2} \mathrm{Cl}_{2}(16 \mathrm{~mL})$, was added Dess-Martin periodinane $(1.32 \mathrm{~g}, 3.1 \mathrm{mmol})$. The mixture was stirred for $13 \mathrm{~h}$ at room temperature under an argon atmosphere. Subsequently, the reaction was quenched with sat. aq. $\mathrm{NaHCO}_{3}$ and the organic layer was separated. The aqueous layer was extracted with $\mathrm{CH}_{2} \mathrm{Cl}_{2}$. The combined organic layer was dried over $\mathrm{MgSO}_{4}$. After filtration, the solvent was evaporated under reduced pressure. The crude product was purified by silica gel column chromatography $($ EtOAc/hexane $=1 / 4)$ to give $\mathbf{1} \mathbf{i}$ as a colorless oil (460 mg, 77\% yield).

The spectral data of $1 \mathbf{i}$ was identical with the reported values ${ }^{5}$.

\section{6-2. Synthesis and Characterization of Carboxylic Acids}

Propionic acid (2a) was purified by distillation from propionic anhydride. $\mathbf{2} \mathbf{h}^{6}$ was prepared by following the same procedure as described in the literature. $\mathbf{2} \mathbf{d}$ and $\mathbf{2} \mathbf{m}$ were prepared by following the procedures shown below. Acetic acid (2j) was purified by distillation from $\mathrm{Ac}_{2} \mathrm{O}$ and $\mathrm{KMnO}_{4}$. Other carboxylic acids were purchased and used without further purification.

\footnotetext{
${ }^{3}$ Schpector, J. Z.; Madureira, L. S.; Wulf, G. D.; Stefani, H. A.; Vasconcelos, S. N. S.; Ng, S. W.; Tiekink, E. R. T. Molecules 2014, 19, 1990.

${ }^{4}$ Peixoto, P. A.; Jean, A.; Maddaluno, J.; Paolis, M. D. Angew. Chem. Int. Ed. 2013, 52, 6971.

${ }^{5}$ Daher, R.; Coinçon, M.; Fonvielle, M.; Gest, P. M.; Guerin, M. E.; Jackson, M.; Syngusch, J.; Therisod, M. J. Med. Chem. 2010, 53, 7836.

${ }^{6}$ Hardouin, C.; Kelso, M. J.; Romero, F. A.; Rayl, T. J.; Leung, D.; Hwang, I.; Cravatt, B. F.; Boger, D. L. J. Med. Chem. 2007, 50, 3359.
} 


\section{Preparation of Carboxylic Acids}

(2d)

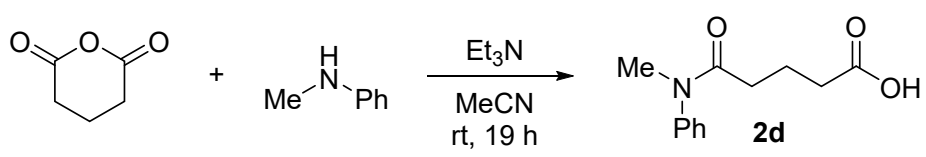

To a solution of $N$-methylaniline $(3.3 \mathrm{~mL}, 30 \mathrm{mmol})$ in $30 \mathrm{~mL}$ of $\mathrm{MeCN}$, were added triethylamine $(4.2 \mathrm{~mL}, 30 \mathrm{mmol})$ and glutaric anhydride $(5.1 \mathrm{~g}, 45 \mathrm{mmol})$. The solution was stirred for $19 \mathrm{~h}$ at room temperature under an argon atmosphere. The solvent was evaporated under reduced pressure and the remaining oil was purified by silica gel column chromatography (EtOAc/hexane $=$ $1 / 2$ to EtOAc). The resulting solid was further purified by washing $\left(\mathrm{Et}_{2} \mathrm{O} /\right.$ hexane $\left.=1 / 10\right)$ to afford $\mathbf{2 d}$ as a white solid (2.8 $\mathrm{g}, 42 \%$ yield).

\section{5-(Methyl(phenyl)amino)-5-oxopentanoic acid (2d)}

$\mathrm{R}_{\mathrm{f}}=0.15($ EtOAc/hexane $=1 / 1) ;{ }^{1} \mathrm{H}$ NMR $\left(400 \mathrm{MHz}, \mathrm{CDCl}_{3}\right): \delta 1.88(\mathrm{tt}, J=7.2,7.2 \mathrm{~Hz}, 2 \mathrm{H}), 2.15$ $(\mathrm{t}, J=7.2 \mathrm{~Hz}, 2 \mathrm{H}), 2.33(\mathrm{t}, J=7.2 \mathrm{~Hz}, 2 \mathrm{H}), 3.26(\mathrm{~s}, 3 \mathrm{H}), 7.14-7.19(\mathrm{~m}, 2 \mathrm{H}), 7.30-7.44(\mathrm{~m}, 3 \mathrm{H}) ;{ }^{13} \mathrm{C}$ NMR (100 MHz, $\left.\mathrm{CDCl}_{3}\right): \delta 20.4,32.9,33.2,37.4,127.2,128.0,129.8,143.7,172.5,178.0$; IR (KBr): 2936, 2625, 1718, 1621, 1496, 1391, 1247, 1122, $703 \mathrm{~cm}^{-1}$; LRMS (ESI): m/z 244 [M+Na] ; HRMS (ESI): $\mathrm{m} / \mathrm{z}$ calcd for $\mathrm{C}_{12} \mathrm{H}_{15} \mathrm{NO}_{3}[\mathrm{M}+\mathrm{Na}]^{+} 244.0950$ Found 244.0947.

(2m)
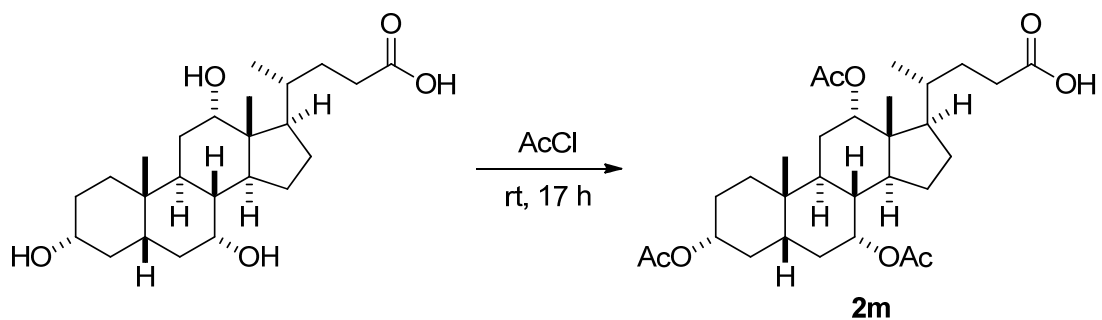

Cholic acid $(1.0 \mathrm{~g}, 2.5 \mathrm{mmol})$ was added to $\mathrm{AcCl}(9.8 \mathrm{~mL})$ at room temperature. After stirring for $17 \mathrm{~h}$, excess amount of $\mathrm{AcCl}$ was removed by evaporation. To a resulting mixture was added EtOAc/ $\mathrm{H}_{2} \mathrm{O}(15 \mathrm{~mL} / 15 \mathrm{~mL})$. The mixture was stirred for $5 \mathrm{~h}$ at room temperature. The organic layer was separated and the aqueous layer was extracted with EtOAc. The combined organic layer was washed with brine, and dried over $\mathrm{Na}_{2} \mathrm{SO}_{4}$. After filtration, the solvent was evaporated under reduced pressure to afford $\mathbf{2 m}$ as a white solid (1.3 g, 99\% yield).

(R)-4-((3R,5S,7R,8R,9S,10S,12S,13R,14S,17R)-3,7,12-Triacetoxy-10,13-dimethylhexadecahydro -1H-cyclopenta[a]phenanthren-17-yl)pentanoic acid (2m) $\mathrm{R}_{\mathrm{f}}=0.23($ EtOAc/hexane $=1 / 2) ;{ }^{1} \mathrm{H}$ NMR $\left(500 \mathrm{MHz}, \mathrm{CDCl}_{3}\right): \delta 0.71(\mathrm{~s}, 3 \mathrm{H}), 0.81(\mathrm{~d}, J=6.3 \mathrm{~Hz}$, $3 \mathrm{H}), 0.90(\mathrm{~s}, 3 \mathrm{H}), 1.02-1.13(\mathrm{~m}, 2 \mathrm{H}), 1.20-1.35(\mathrm{~m}, 3 \mathrm{H}), 1.37-1.43(\mathrm{~m}, 2 \mathrm{H}), 1.47-1.53(\mathrm{~m}, 2 \mathrm{H})$, 1.56-1.67 (m, 5H), 1.71-1.88 (m, 5H), 1.91-2.02 (m, 3H), $2.03(\mathrm{~s}, 3 \mathrm{H}), 2.07(\mathrm{~s}, 3 \mathrm{H}), 2.12(\mathrm{~s}, 3 \mathrm{H})$, 
2.19-2.26 (m, 1H), 2.34-2.40 (m, 1H), 4.53-4.59 (m, 1H), 4.89 (d, $J=2.3 \mathrm{~Hz}, 1 \mathrm{H}), 5.07(\mathrm{~s}, 1 \mathrm{H}) ;{ }^{13} \mathrm{C}$ NMR (125 MHz, $\left.\mathrm{CDCl}_{3}\right): \delta$ 12.2, 17.4, 21.4, 21.4, 21.6, 22.5, 22.7, 25.5, 26.8, 27.1, 28.8, 30.5, 30.8, $31.2,34.3,34.5,34.5,34.6,37.7,40.9,43.3,45.0,47.3$, 70.7, 74.1, 75.3, 170.4, 170.5, 170.6, 179.7; IR (neat): 2944, 1733, 1378, 1249, 1025, $756 \mathrm{~cm}^{-1}$; LRMS (ESI): m/z 557.6 [M+Na] $]^{+}$HRMS (ESI): $\mathrm{m} / \mathrm{z}$ calcd for $\mathrm{C}_{30} \mathrm{H}_{46} \mathrm{O}_{8}[\mathrm{M}+\mathrm{Na}]^{+} 557.3090$ Found 557.3090; $[\alpha]_{\mathrm{D}}^{22.9}=+62.8\left(c=0.34, \mathrm{CHCl}_{3}\right)$.

\section{6-3. Synthesis and Characterization of Ligands (Ts-Amino Acids, L1 and L2)}

Ts-L-Val, Ts-L-Leu and other Ts-amino acids were prepared by following the same procedure as described in the literature ${ }^{7} . \mathbf{L} 1$ and $\mathbf{L} 2$ were prepared by following the procedure shown below. Other ligands were purchased and used without further purification.

\section{Preparation of L1 and L2}

(L1)<smiles>COC(=O)C(NS(=O)(=O)c1c(F)c(F)c(F)c(F)c1F)C(C)C(C)(O)C(=O)O</smiles>

Following the reported procedure, $\mathrm{C}_{6} \mathrm{~F}_{5}-\mathrm{SO}_{2}$-Val-OMe was prepared ${ }^{8}$. To a solution of $\mathrm{C}_{6} \mathrm{~F}_{5}-\mathrm{SO}_{2}$-Val-OMe $(4.7 \mathrm{~g}, 13 \mathrm{mmol})$ in $\mathrm{MeOH}(20 \mathrm{~mL})$, was added aqueous solution of $\mathrm{LiOH}$ ( $\mathrm{LiOH} \cdot \mathrm{H}_{2} \mathrm{O}(39 \mathrm{mmol})$ in $\left.\mathrm{H}_{2} \mathrm{O}(20 \mathrm{~mL})\right)$. The mixture was stirred overnight at $50{ }^{\circ} \mathrm{C}$, then cooled to room temperature. After the mixture was washed with $\mathrm{Et}_{2} \mathrm{O}$, aqueous layer was acidified with conc. $\mathrm{HCl}$, and extracted with EtOAc. The combined organic layer was dried over $\mathrm{MgSO}_{4}$. After filtration, the solvent was evaporated under reduced pressure. The crude product was purified by silica gel column chromatography $($ EtOAc/hexane $=1 / 5$ to $1 / 1)$ to give $\mathbf{L 1}$ as a white powder $(1.7 \mathrm{~g}, 36 \%$ yield). The unexpected ipso-substitution of 4-position of $\mathrm{C}_{6} \mathrm{~F}_{5} \mathrm{SO}_{2}$-group by $\mathrm{MeO}$-group occurred simultaneously with the hydrolysis of the methyl esters.

\section{((2,3,5,6-Tetrafluoro-4-methoxyphenyl)sulfonyl)-L-valine (L1)}

A white solid; Yield: $36 \% ; \mathrm{R}_{\mathrm{f}}=0.35\left(\mathrm{MeOH} / \mathrm{CH}_{2} \mathrm{Cl}_{2}=1 / 10\right) ;{ }^{1} \mathrm{H}$ NMR (500 MHz, acetone- $\left.d_{6}\right): \delta$ $0.96(\mathrm{~d}, J=6.9 \mathrm{~Hz}, 3 \mathrm{H}), 1.02(\mathrm{~d}, J=6.9 \mathrm{~Hz}, 3 \mathrm{H}), 2.19-2.28(\mathrm{~m}, 1 \mathrm{H}), 3.93(\mathrm{~d}, J=5.2 \mathrm{~Hz}, 1 \mathrm{H}), 4.22$ $\left(\mathrm{t},{ }^{5} J_{\mathrm{F}-\mathrm{H}}=1.7 \mathrm{~Hz}, 3 \mathrm{H}\right) ;{ }^{13} \mathrm{C} \mathrm{NMR}\left(125 \mathrm{MHz}\right.$, acetone- $\left.d_{6}\right): \delta 17.7,19.2,31.3,62.2,62.5\left(\mathrm{t},{ }^{4} J_{\mathrm{F}-\mathrm{C}}=\right.$ $3.60 \mathrm{~Hz}), 114.3\left(\mathrm{t},{ }^{2} J_{\mathrm{F}-\mathrm{C}}=15.6 \mathrm{~Hz}\right), 141.1\left(\mathrm{dddd},{ }^{1} J_{\mathrm{F}-\mathrm{C}}=248.0 \mathrm{~Hz},{ }^{2} J_{\mathrm{F}-\mathrm{C}}=14.4 \mathrm{~Hz},{ }^{3} J_{\mathrm{F}-\mathrm{C}}=4.8 \mathrm{~Hz}\right.$, $\left.{ }^{4} J_{\mathrm{F}-\mathrm{C}}=3.6 \mathrm{~Hz}\right), 142.4\left(\mathrm{tt},{ }^{2} J_{\mathrm{F}-\mathrm{C}}=10.8 \mathrm{~Hz},{ }^{3} J_{\mathrm{F}-\mathrm{C}}=3.6 \mathrm{~Hz}\right), 145.4\left(\mathrm{dddd},{ }^{1} J_{\mathrm{F}-\mathrm{C}}=254.0 \mathrm{~Hz},{ }^{2} J_{\mathrm{F}-\mathrm{C}}=13.2\right.$

\footnotetext{
${ }^{7}$ Västilä, P.; Pastor, I. S.; Adolfsson, H. J. Org. Chem. 2005, 70, 2921.

8 The syntheses of similar compounds were reported. Penso, M.; Albanese, D.; Landini, D.; Lupi, V.; Tagliabue, A. J. Org. Chem. 2008, 73, 6686.
} 
$\left.\mathrm{Hz},{ }^{3} J_{\mathrm{F}-\mathrm{C}}=6.0 \mathrm{~Hz},{ }^{4} J_{\mathrm{F}-\mathrm{C}}=3.6 \mathrm{~Hz}\right), 171.8 ;{ }^{19} \mathrm{~F} \mathrm{NMR}\left(368 \mathrm{MHz}, \mathrm{CDCl}_{3}\right): \delta-157.0(\mathrm{~d}, J=16.9 \mathrm{~Hz}, 2 \mathrm{~F})$, -139.0 (d, $J=16.9 \mathrm{~Hz}, 2 \mathrm{~F}$ ); IR (KBr): 3352, 3202, 2970, 1639, 1495, 1397, 1201, 1125, $1005 \mathrm{~cm}^{-1}$; LRMS (ESI): m/z $382[\mathrm{M}+\mathrm{Na}]^{+}$; HRMS (ESI): $\mathrm{m} / \mathrm{z}$ calcd for $\mathrm{C}_{12} \mathrm{H}_{13} \mathrm{~F}_{4} \mathrm{NO}_{5} \mathrm{~S}[\mathrm{M}+\mathrm{Na}]^{+} 382.0348$ Found 382.0349; $[\alpha]_{\mathrm{D}}^{22.7}=+29.2(c=0.76, \mathrm{MeOH})$.

(L2)

Following the reported procedure, 2,6- $\mathrm{F}_{2}-\mathrm{C}_{6} \mathrm{H}_{3} \mathrm{SO}_{2}-\mathrm{Val}-\mathrm{O}^{t} \mathrm{Bu}$ was prepared ${ }^{8}$.

tert-Butyl ((2,6-difluorophenyl)sulfonyl)-L-valinate (S1)

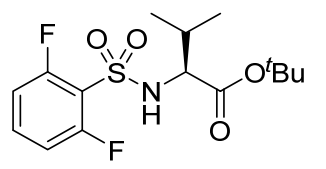

A white solid; Yield: $85 \% ; \mathrm{R}_{\mathrm{f}}=0.32($ EtOAc/hexane $=1 / 4) ;{ }^{1} \mathrm{H}$ NMR $\left(500 \mathrm{MHz}, \mathrm{CDCl}_{3}\right): \delta 0.89(\mathrm{~d}$, $J=6.9 \mathrm{~Hz}, 3 \mathrm{H}), 1.02$ (d, $J=6.3 \mathrm{~Hz}, 3 \mathrm{H}), 1.26(\mathrm{~s}, 9 \mathrm{H}), 2.08-2.15$ (m, 1H), 3.89 (dd, $J=9.7,4.6 \mathrm{~Hz}$, $1 \mathrm{H}), 5.54(\mathrm{~d}, J=9.7 \mathrm{~Hz}, \mathrm{NH}), 7.00(\mathrm{t}, J=8.6 \mathrm{~Hz}, 2 \mathrm{H}), 7.45-7.51(\mathrm{~m}, 1 \mathrm{H}) ;{ }^{13} \mathrm{C} \mathrm{NMR}(125 \mathrm{MHz}$, $\left.\mathrm{CDCl}_{3}\right): \delta 17.1,18.9,27.7,31.6,61.5,82.5,112.9\left(\mathrm{dd},{ }^{2} J_{\mathrm{F}-\mathrm{C}}=22.7 \mathrm{~Hz},{ }^{4} J_{\mathrm{F}-\mathrm{C}}=3.6 \mathrm{~Hz}\right), 117.9\left(\mathrm{t},{ }^{2} J_{\mathrm{F}-\mathrm{C}}\right.$ $=15.5 \mathrm{~Hz}), 134.4\left(\mathrm{t},{ }^{3} J_{\mathrm{F}-\mathrm{C}}=10.7 \mathrm{~Hz}\right), 159.6\left(\mathrm{dd},{ }^{1} J_{\mathrm{F}-\mathrm{C}}=257.5 \mathrm{~Hz},{ }^{3} J_{\mathrm{F}-\mathrm{C}}=3.6 \mathrm{~Hz}\right), 169.9 ;{ }^{19} \mathrm{~F} \mathrm{NMR}$ (368 MHz, $\mathrm{CDCl}_{3}$ ): $\delta$-107.2 (s, 2F); IR (KBr): 3336, 2969, 1724, 1614, 1473, 1362, 1003, $799 \mathrm{~cm}^{-1}$; LRMS (ESI): $\mathrm{m} / \mathrm{z} 372[\mathrm{M}+\mathrm{Na}]^{+}$; HRMS (ESI): $\mathrm{m} / \mathrm{z}$ calcd for $\mathrm{C}_{15} \mathrm{H}_{21} \mathrm{~F}_{2} \mathrm{NO}_{4} \mathrm{~S}[\mathrm{M}+\mathrm{Na}]^{+} 372.1057$ Found $372.1062 ;[\alpha]_{\mathrm{D}}^{21.1}=+39.8\left(c=1.0, \mathrm{CH}_{3} \mathrm{Cl}\right)$.

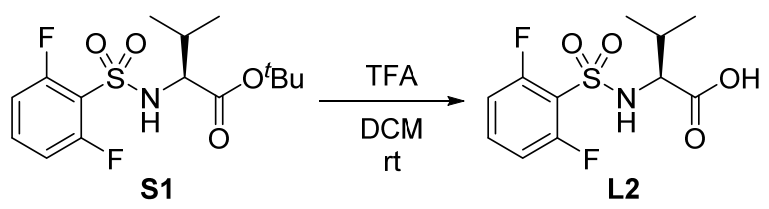

To a solution of 2,6- $\mathrm{F}_{2}-\mathrm{C}_{6} \mathrm{H}_{3} \mathrm{SO}_{2}-\mathrm{Val}-\mathrm{O}{ }^{t} \mathrm{Bu}(\mathrm{S1})(4.3 \mathrm{~g}, 12 \mathrm{mmol})$ in $\mathrm{CH}_{2} \mathrm{Cl}_{2}(24 \mathrm{~mL})$, was added TFA $(30 \mathrm{~mL}, 370 \mathrm{mmol})$ at $0{ }^{\circ} \mathrm{C}$. The mixture was stirred for $14 \mathrm{~h}$ at room temperature. The solvent was evaporated under reduced pressure to give $\mathbf{L} 2$ as a white powder (3.6 g, 100\% yield).

((2,6-Difluorophenyl)sulfonyl)-L-valine (L2)

A white solid; Yield: $100 \% ; \mathrm{R}_{\mathrm{f}}=0.26\left(\mathrm{MeOH} / \mathrm{CH}_{2} \mathrm{Cl}_{2}=1 / 10\right) ;{ }^{1} \mathrm{H}$ NMR $\left(500 \mathrm{MHz}, \mathrm{CDCl}_{3}\right): \delta 0.91$ (d, $J=6.9 \mathrm{~Hz}, 3 \mathrm{H}), 1.00(\mathrm{~d}, J=6.9 \mathrm{~Hz}, 3 \mathrm{H}), 2.17-2.23(\mathrm{~m}, 1 \mathrm{H}), 4.07$ (dd, $J=9.2,4.0 \mathrm{~Hz}, 1 \mathrm{H}), 5.64$ $(\mathrm{d}, J=8.6 \mathrm{~Hz}, \mathrm{NH}), 7.01(\mathrm{t}, J=8.6 \mathrm{~Hz}, 2 \mathrm{H}), 7.49-7.59(\mathrm{~m}, 1 \mathrm{H}) ;{ }^{13} \mathrm{C} \mathrm{NMR}\left(125 \mathrm{MHz}, \mathrm{CDCl}_{3}\right): \delta$ $17.1,18.9,31.2,61.2,113.0\left(\mathrm{dd},{ }^{2} J_{\mathrm{F}-\mathrm{C}}=23.9 \mathrm{~Hz},{ }^{4} J_{\mathrm{F}-\mathrm{C}}=3.6 \mathrm{~Hz}\right), 118.0\left(\mathrm{t},{ }^{2} J_{\mathrm{F}-\mathrm{C}}=15.5 \mathrm{~Hz}\right), 134.8(\mathrm{t}$, $\left.{ }^{3} J_{\mathrm{F}-\mathrm{C}}=10.7 \mathrm{~Hz}\right), 159.4\left(\mathrm{dd},{ }^{1} J_{\mathrm{F}-\mathrm{C}}=257.5 \mathrm{~Hz},{ }^{3} J_{\mathrm{F}-\mathrm{C}}=3.6 \mathrm{~Hz}\right), 176.0 ;{ }^{19} \mathrm{~F} \mathrm{NMR}\left(368 \mathrm{MHz}, \mathrm{CDCl}_{3}\right): \delta$ -107.5 (s, 2F); IR (KBr): 3337, 3290, 2970, 1719, 1613, 1472, 1351, 1008, $796 \mathrm{~cm}^{-1}$; LRMS (ESI): $\mathrm{m} / \mathrm{z} 316[\mathrm{M}+\mathrm{Na}]^{+}$; HRMS (ESI): $\mathrm{m} / \mathrm{z}$ calcd for $\mathrm{C}_{11} \mathrm{H}_{13} \mathrm{~F}_{2} \mathrm{NO}_{4} \mathrm{~S}[\mathrm{M}+\mathrm{Na}]^{+} 316.0431$ Found 316.0421; $[\alpha]_{\mathrm{D}}^{21.2}=+15.1(c=0.62, \mathrm{MeOH})$. 


\section{6-4. Characterization of Products (Table 2, 3)}

syn-Methyl 3-hydroxy-2-methyl-5-phenylpentanoate (5ba: methyl ester of 3ba)<smiles>COC(=O)C(C)CCc1ccccc1</smiles>

A colorless oil; Yield: 83\%; syn/anti $=8.2 / 1$; (ligand: L2, DBU: 2.5 equiv, $12 \mathrm{~h}$ ); $\mathrm{R}_{\mathrm{f}}=0.30$ $($ EtOAc/hexane $=1 / 4) ;{ }^{1} \mathrm{H}$ NMR $\left(500 \mathrm{MHz}, \mathrm{CDCl}_{3}\right): \delta 1.19(\mathrm{~s}, J=7.5 \mathrm{~Hz}, 3 \mathrm{H}), 1.64-1.71(\mathrm{~m}, 1 \mathrm{H})$, 1.77-1.85 (m, 1H), 2.53-2.59 (m, 1H), 2.63-2.69 (m, 1H), 2.83-2.89 (m, 1H), $3.70(\mathrm{~s}, 3 \mathrm{H}), 3.92(\mathrm{dt}, J$ $=9.8,2.5 \mathrm{~Hz}, 1 \mathrm{H}), 7.18-7.21(\mathrm{~m}, 3 \mathrm{H}), 7.27-7.30(\mathrm{~m}, 2 \mathrm{H}) ;{ }^{13} \mathrm{C} \mathrm{NMR}\left(125 \mathrm{MHz}, \mathrm{CDCl}_{3}\right): \delta 10.7,32.3$, 35.5, 44.3, 51.8, 71.0, 125.9, 128.4, 128.4, 141.8, 176.5; IR (neat): 3502, 2950, 1733, 1456, 1200, 1044, $750 \mathrm{~cm}^{-1}$; LRMS (ESI): m/z $245[\mathrm{M}+\mathrm{Na}]^{+}$; HRMS (ESI): $\mathrm{m} / \mathrm{z}$ calcd for $\mathrm{C}_{13} \mathrm{H}_{18} \mathrm{O}_{3}[\mathrm{M}+\mathrm{Na}]^{+}$ 245.1154 Found 245.1161.

syn-Methyl 3-(4-chlorophenyl)-3-hydroxy-2-methylpropanoate (5ca: methyl ester of 3ca)<smiles>CC(=O)C(C)[C@H](O)c1ccc(Cl)cc1</smiles>

A colorless oil; Yield: 95\%; syn/anti $=14 / 1 ; \mathrm{R}_{\mathrm{f}}=0.33($ EtOAc/hexane $=1 / 4) ;{ }^{1} \mathrm{H}$ NMR $(500 \mathrm{MHz}$, $\left.\mathrm{CDCl}_{3}\right): \delta 1.10(\mathrm{~d}, J=6.9 \mathrm{~Hz}, 3 \mathrm{H}), 2.72-2.77(\mathrm{~m}, 1 \mathrm{H}), 3.69(\mathrm{~s}, 3 \mathrm{H}), 5.09(\mathrm{~d}, J=4.0 \mathrm{~Hz}, 1 \mathrm{H}), 7.27(\mathrm{~d}$, $J=8.6 \mathrm{~Hz}, 2 \mathrm{H}), 7.32(\mathrm{~d}, J=8.6 \mathrm{~Hz}, 2 \mathrm{H}) ;{ }^{13} \mathrm{C} \mathrm{NMR}\left(125 \mathrm{MHz}, \mathrm{CDCl}_{3}\right): \delta 10.5,46.1,52.0,72.8$, 127.3, 128.4, 133.2, 139.8, 176.2; IR (neat): 3482, 2952, 1732, 1492, 1200, 1090, $813 \mathrm{~cm}^{-1}$; LRMS (ESI): $\mathrm{m} / \mathrm{z} 251[\mathrm{M}+\mathrm{Na}]^{+}$; HRMS (ESI): $\mathrm{m} / \mathrm{z}$ calcd for $\mathrm{C}_{11} \mathrm{H}_{13} \mathrm{ClO}_{3}[\mathrm{M}+\mathrm{Na}]^{+} 251.0451$ Found 251.0458 .

syn-Methyl 3-hydroxy-2-methyl-3-(4-(trifluoromethyl)phenyl)propanoate (5da: methyl ester of 3da)<smiles>COC(=O)[C@H](C)[C@H](O)c1ccc(C(F)(F)F)cc1</smiles>

A colorless oil; Yield: 96\%; syn/anti $=14 / 1$; (toluene: $2.0 \mathrm{~mL}) ; \mathrm{R}_{\mathrm{f}}=0.36($ EtOAc/hexane $=1 / 4) ;{ }^{1} \mathrm{H}$ NMR (500 MHz, $\left.\mathrm{CDCl}_{3}\right): \delta 1.09$ (d, J=7.5 Hz, 3H), 2.76-2.81 (m, 1H), $3.23(\mathrm{~s}, 1 \mathrm{H}), 3.70(\mathrm{~s}, 3 \mathrm{H})$, $5.18(\mathrm{~d}, J=2.9 \mathrm{~Hz}, 1 \mathrm{H}), 7.46(\mathrm{~d}, J=8.6 \mathrm{~Hz}, 2 \mathrm{H}), 7.60(\mathrm{~d}, J=8.6 \mathrm{~Hz}, 2 \mathrm{H}) ;{ }^{13} \mathrm{C}$ NMR $(125 \mathrm{MHz}$, $\left.\mathrm{CDCl}_{3}\right): \delta 10.3,45.9,52.0,72.8,124.1\left(\mathrm{q},{ }^{1} J_{\mathrm{F}-\mathrm{C}}=269.5 \mathrm{~Hz}\right), 125.2\left(\mathrm{q},{ }^{3} J_{\mathrm{F}-\mathrm{C}}=3.6 \mathrm{~Hz}\right), 126.3,129.6$ $\left(\mathrm{q},{ }^{1} J_{\mathrm{F}-\mathrm{C}}=32.2 \mathrm{~Hz}\right), 145.3,176.1 ;{ }^{19} \mathrm{~F}$ NMR $\left(368 \mathrm{MHz}, \mathrm{CDCl}_{3}\right): \delta-63.1$ (s, 3F); IR (neat): 3481, 2954, 1723, 1327, 11123, $844 \mathrm{~cm}^{-1}$; LRMS (ESI): m/z 285 [M+Na] $]^{+}$HRMS (ESI): m/z calcd for 
$\mathrm{C}_{12} \mathrm{H}_{13} \mathrm{~F}_{3} \mathrm{O}_{3}[\mathrm{M}+\mathrm{Na}]^{+}$285.0714 Found 285.0720.

syn-Methyl 3-hydroxy-2-methyl-3-(4-(pivaloyloxy)phenyl)propanoate (5ea: methyl ester of 3ea)<smiles>CCCOc1ccc([C@@H](O)[C@@H](C)C(=O)OC)cc1</smiles>

A colorless oil; Yield: $89 \%$; syn/anti $=11 / 1 ;\left(\mathrm{DBU}: 2.5\right.$ equiv, $\left.\mathrm{SiO}_{2}: \mathrm{CH}_{2} \mathrm{Cl}_{2}\right) ; \mathrm{R}_{\mathrm{f}}=0.22$ $($ EtOAc/hexane $=1 / 4) ;{ }^{1} \mathrm{H}$ NMR $\left(500 \mathrm{MHz}, \mathrm{CDCl}_{3}\right): \delta 1.12(\mathrm{~d}, J=6.9 \mathrm{~Hz}, 3 \mathrm{H}), 1.35(\mathrm{~s}, 9 \mathrm{H})$, 2.74-2.79 (m, 1H), 2.99 (d, $J=2.3 \mathrm{~Hz}, 1 \mathrm{H}), 3.67$ (s, 3H), 5.10 (s, 1H), 7.03 (d, $J=8.6 \mathrm{~Hz}, 2 \mathrm{H}), 7.34$ (d, $J=8.6 \mathrm{~Hz}, 2 \mathrm{H}) ;{ }^{13} \mathrm{C}$ NMR $\left(125 \mathrm{MHz}, \mathrm{CDCl}_{3}\right): \delta 10.6,27.1,39.0,46.3,51.9,73.1,121.3,126.9$, 138.7, 150.3, 176.2, 177.1; IR (neat): 3501, 2977, 1747, 1508, 1200, 1119, $898 \mathrm{~cm}^{-1}$; LRMS (ESI): $\mathrm{m} / \mathrm{z} 317[\mathrm{M}+\mathrm{Na}]^{+}$; HRMS (ESI): $\mathrm{m} / \mathrm{z}$ calcd for $\mathrm{C}_{16} \mathrm{H}_{22} \mathrm{O}_{5}[\mathrm{M}+\mathrm{Na}]^{+} 317.1365$ Found 317.1353.

syn-Methyl 3-hydroxy-3-(4-methoxyphenyl)-2-methylpropanoate (5fa: methyl ester of 3fa)<smiles>COC(=O)[C@H](O)[C@H](C)c1ccc(OC)cc1</smiles>

A colorless oil; Yield: 48\%; syn/anti $=6.5 / 1 ;(\mathrm{GPC}) ;{ }^{1} \mathrm{H}$ NMR $\left(500 \mathrm{MHz}, \mathrm{CDCl}_{3}\right): \delta 1.14(\mathrm{~d}, J=7.5$ $\mathrm{Hz}, 3 \mathrm{H}), 2.73-2.79(\mathrm{~m}, 1 \mathrm{H}), 2.83$ (d, $J=2.9 \mathrm{~Hz}, \mathrm{OH}), 3.66(\mathrm{~s}, 3 \mathrm{H}), 3.80(\mathrm{~s}, 3 \mathrm{H}), 5.02$ (t, $J=2.9 \mathrm{~Hz}$, $1 \mathrm{H}), 6.86(\mathrm{~d}, J=8.6 \mathrm{~Hz}, 2 \mathrm{H}), 7.25(\mathrm{~d}, J=8.6 \mathrm{~Hz}, 2 \mathrm{H}) ;{ }^{13} \mathrm{C} \mathrm{NMR}\left(125 \mathrm{MHz}, \mathrm{CDCl}_{3}\right): \delta 11.0,46.5$, 51.8, 55.2, 73.4, 113.6, 127.1, 133.5, 158.9, 176.2; IR (neat): 3501, 2952, 1733, 1514, 1249, 1034 , $833 \mathrm{~cm}^{-1}$; LRMS (ESI): $\mathrm{m} / \mathrm{z} 247$ [M+Na] $]^{+}$; HRMS (ESI): $\mathrm{m} / \mathrm{z}$ calcd for $\mathrm{C}_{12} \mathrm{H}_{16} \mathrm{O}_{4}[\mathrm{M}+\mathrm{Na}]^{+} 247.0946$ Found 247.0945.

syn-Methyl 3-hydroxy-2-methyl-3-(thiophen-2-yl)propanoate (5ga: methyl ester of 3ga)<smiles>COC(=O)C(C)C(O)c1cccs1</smiles>

A colorless oil; Yield: 62\%; syn/anti $=11 / 1$; (toluene: $2.0 \mathrm{~mL}) ; \mathrm{R}_{\mathrm{f}}=0.30($ EtOAc/hexane $=1 / 4) ;{ }^{1} \mathrm{H}$ $\operatorname{NMR}\left(500 \mathrm{MHz}, \mathrm{CDCl}_{3}\right): \delta 1.23(\mathrm{~d}, J=6.9 \mathrm{~Hz}, 3 \mathrm{H}), 2.86-2.91(\mathrm{~m}, 1 \mathrm{H}), 3.09(\mathrm{~d}, J=4.0 \mathrm{~Hz}, 1 \mathrm{H})$, $3.70(\mathrm{~s}, 3 \mathrm{H}), 5.30(\mathrm{t}, J=4.0 \mathrm{~Hz}, 1 \mathrm{H}), 6.94-6.98(\mathrm{~m}, 2 \mathrm{H}), 7.24(\mathrm{dd}, J=5.2,1.2 \mathrm{~Hz}, 1 \mathrm{H}) ;{ }^{13} \mathrm{C} \mathrm{NMR}$ (125 MHz, $\left.\mathrm{CDCl}_{3}\right): \delta 11.4,46.7,52.0,70.8,123.7,124.5,126.7,145.2,175.6$; IR (neat): 3481, 2951, 1733, 1457, 1201, 1035, $704 \mathrm{~cm}^{-1}$; LRMS (ESI): m/z 223 [M+Na] ; HRMS (ESI): m/z calcd for $\mathrm{C}_{9} \mathrm{H}_{12} \mathrm{O}_{3} \mathrm{~S}[\mathrm{M}+\mathrm{Na}]^{+} 223.0405$ Found 223.0398. 
syn-Methyl 3-hydroxy-2-methyl-3-(1-tosyl-1H-indol-3-yl)propanoate (5ha: methyl ester of 3ha)<smiles></smiles>

A white solid; Yield: 82\%; syn/anti $=>20 / 1 ;\left(\mathrm{SiO}_{2}\right.$ : EtOAc/hexane $\left.=1 / 4\right) ; \mathrm{R}_{\mathrm{f}}=0.09($ EtOAc/hexane $=1 / 4) ;{ }^{1} \mathrm{H}$ NMR $\left(500 \mathrm{MHz}, \mathrm{CDCl}_{3}\right): \delta 1.13(\mathrm{~d}, J=7.5 \mathrm{~Hz}, 3 \mathrm{H}), 2.33(\mathrm{~s}, 3 \mathrm{H}), 2.90-2.95(\mathrm{~m}, 1 \mathrm{H})$, $3.02(\mathrm{~d}, J=3.5 \mathrm{~Hz}, \mathrm{OH}), 3.69$ (s, 3H), 5.37 (t, $J=3.5 \mathrm{~Hz}, 1 \mathrm{H}), 7.20-7.22(\mathrm{~m}, 3 \mathrm{H}), 7.31$ (t, $J=8.0 \mathrm{~Hz}$, $1 \mathrm{H}), 7.51(\mathrm{~d}, J=8.0 \mathrm{~Hz}, 1 \mathrm{H}), 7.59(\mathrm{~s}, 1 \mathrm{H}), 7.75(\mathrm{~d}, J=8.0 \mathrm{~Hz}, 1 \mathrm{H}), 7.99(\mathrm{~d}, J=8.0 \mathrm{~Hz}, 1 \mathrm{H}) ;{ }^{13} \mathrm{C}$ NMR $\left(125 \mathrm{MHz}, \mathrm{CDCl}_{3}\right): \delta 10.7,21.5,44.2,52.0,68.0,113.9,119.8,122.9,123.2,123.7,124.8$, 126.8, 128.5, 129.8, 135.1, 135.3, 144.9, 176.2; IR (KBr): 3491, 2948, 1740, 1367, 1172, 968, 749 $\mathrm{cm}^{-1}$; LRMS (ESI): $\mathrm{m} / \mathrm{z} 410[\mathrm{M}+\mathrm{Na}]^{+}$; HRMS (ESI): $\mathrm{m} / \mathrm{z}$ calcd for $\mathrm{C}_{20} \mathrm{H}_{21} \mathrm{NO}_{5} \mathrm{~S}[\mathrm{M}+\mathrm{Na}]^{+} 410.1038$ Found 410.1043 .

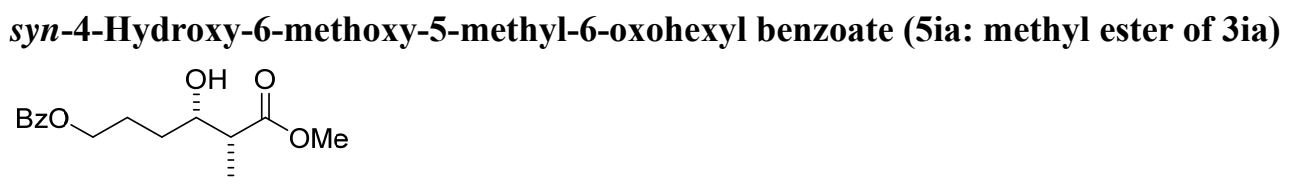

A colorless oil; Yield: 86\%; syn/anti =12/1; (ligand: L2, solvent: $\mathrm{C}_{6} \mathrm{H}_{5} \mathrm{CF}_{3}, 12 \mathrm{~h}, \mathrm{GPC}$ ); $\mathrm{R}_{\mathrm{f}}=0.15$ $\left(\mathrm{CH}_{2} \mathrm{Cl}_{2}\right) ;{ }^{1} \mathrm{H}$ NMR (500 MHz, $\left.\mathrm{CDCl}_{3}\right): \delta 1.20(\mathrm{~d}, J=7.5 \mathrm{~Hz}, 3 \mathrm{H}), 1.52-1.66(\mathrm{~m}, 3 \mathrm{H}), 1.79-1.90(\mathrm{~m}$, $1 \mathrm{H}), 1.97-2.06(\mathrm{~m}, 1 \mathrm{H}), 2.54-2.60(\mathrm{~m}, 1 \mathrm{H}), 2.63(\mathrm{~d}, J=4.6 \mathrm{~Hz}, \mathrm{OH}), 3.71(\mathrm{~s}, 3 \mathrm{H}), 3.95-3.99(\mathrm{~m}, 1 \mathrm{H})$, 4.32-4.40 (m, 2H), $7.44(\mathrm{t}, J=7.5 \mathrm{~Hz}, 2 \mathrm{H}), 7.56(\mathrm{t}, J=7.5 \mathrm{~Hz}, 1 \mathrm{H}), 8.03(\mathrm{~d}, J=7.5 \mathrm{~Hz}, 2 \mathrm{H}) ;{ }^{13} \mathrm{C}$ NMR (125 MHz, $\left.\mathrm{CDCl}_{3}\right): \delta$ 10.7, 25.4, 30.2, 44.3, 51.9, 64.7, 71.3, 128.3, 129.5, 130.3, 132.9, 166.6, 176.4; IR (neat): 3503, 2952, 1717, 1455, 1277, 1116, $714 \mathrm{~cm}^{-1}$; LRMS (ESI): m/z 303 [M+Na] ; HRMS (ESI): $\mathrm{m} / \mathrm{z}$ calcd for $\mathrm{C}_{15} \mathrm{H}_{20} \mathrm{O}_{5}[\mathrm{M}+\mathrm{Na}]^{+} 303.1208$ Found 303.1209 .

syn-Methyl 3-cyclohexyl-3-hydroxy-2-methylpropanoate (5ja: methyl ester of 3ja)<smiles>COC(=O)C(C)C(O)C1CCCCC1</smiles>

A colorless oil; Yield: $80 \%$; syn/anti $=>20 / 1$; (ligand: L2, solvent: $\mathrm{C}_{6} \mathrm{H}_{5} \mathrm{CF}_{3}, 12 \mathrm{~h}$ ); $\mathrm{R}_{\mathrm{f}}=0.29$ $($ EtOAc/hexane $=1 / 4) ;{ }^{1} \mathrm{H}$ NMR $\left(500 \mathrm{MHz}, \mathrm{CDCl}_{3}\right): \delta 0.90-1.01(\mathrm{~m}, 2 \mathrm{H}), 1.14-1.28(\mathrm{~m}, 6 \mathrm{H})$, $1.31-1.40(\mathrm{~m}, 1 \mathrm{H}), 1.55$ (d, $J=12.4 \mathrm{~Hz}, 1 \mathrm{H}), 1.67$ (d, $J=13.8 \mathrm{~Hz}, 1 \mathrm{H}), 1.71-1.77$ (m, 2H), 2.05 (d, $J$ $=16.1 \mathrm{~Hz}, 1 \mathrm{H}), 2.46(\mathrm{~s}, 1 \mathrm{H}), 2.67(\mathrm{dq}, J=7.5,3.2 \mathrm{~Hz}, 1 \mathrm{H}), 3.63(\mathrm{dd}, J=8.7,3.2 \mathrm{~Hz}, 1 \mathrm{H}), 3.70(\mathrm{~s}$, $3 \mathrm{H}) ;{ }^{13} \mathrm{C}$ NMR $\left(125 \mathrm{MHz}, \mathrm{CDCl}_{3}\right): \delta 9.9,25.8,26.0,26.3,28.9,29.1,40.0,41.1,51.8,75.6$, 177.1; IR (neat): 3502, 2926, 1734, 1450, 1201, $991 \mathrm{~cm}^{-1}$; LRMS (ESI): m/z 223 [M+Na] ; HRMS (ESI): $\mathrm{m} / \mathrm{z}$ calcd for $\mathrm{C}_{11} \mathrm{H}_{20} \mathrm{O}_{3}[\mathrm{M}+\mathrm{Na}]^{+} 223.1310$ Found 223.1300 . 
Methyl 3-hydroxy-2,4,4-trimethylpentanoate (5ka: methyl ester of 3ka)<smiles>COC(=O)[C@H](C)[C@H](O)C(C)(C)C</smiles>

A pale yellow oil; Yield: 35\%; syn/anti=2.6/1; (ligand: L1, solvent: $\mathrm{C}_{6} \mathrm{H}_{5} \mathrm{CF}_{3}, 40$ h, GPC);

The spectral data of $\mathbf{5 k a}$ was identical with the reported values ${ }^{9}$. ${ }^{1} \mathrm{H}$ NMR spectral chart of diastereomixture of $\mathbf{5 k a}$ was shown in Section 7 of this SI.

Methyl (E)-3-hydroxy-2-methyl-5-phenylpent-4-enoate (5la: methyl ester of 3la)<smiles>COC(=O)C(C)[C@H](O)/C=C/c1ccccc1/C=C/[C@@H](O)[C@@H](C)C(=O)OC</smiles>

A colorless oil; Yield: 95\%; syn/anti = 2.1/1; (ligand: L2, DBU: 2.5 equiv, solvent: $\mathrm{C}_{6} \mathrm{H}_{5} \mathrm{CF}_{3}, 12 \mathrm{~h}$ );

(syn)

$\mathrm{R}_{\mathrm{f}}=0.19($ EtOAc/hexane $=1 / 4) ;{ }^{1} \mathrm{H} \mathrm{NMR}\left(500 \mathrm{MHz}, \mathrm{CDCl}_{3}\right): \delta 1.23(\mathrm{~d}, J=6.9 \mathrm{~Hz}, 3 \mathrm{H}), 2.72-2.76$ $(\mathrm{m}, 1 \mathrm{H}), 2.78(\mathrm{~d}, 4.0 \mathrm{~Hz}, 1 \mathrm{H}), 3.72$ (s, 3H), 4.59 (s, 1H), 6.19 (dd, $J=16.1,6.3 \mathrm{~Hz}, 1 \mathrm{H}), 6.66(\mathrm{~d}, J=$ $16.1 \mathrm{~Hz}, 1 \mathrm{H}), 7.24(\mathrm{~d}, J=7.5 \mathrm{~Hz}, 1 \mathrm{H}), 7.32(\mathrm{t}, J=7.5 \mathrm{~Hz}, 2 \mathrm{H}), 7.38(\mathrm{~d}, J=7.5 \mathrm{~Hz}, 2 \mathrm{H}) ;{ }^{13} \mathrm{C}$ NMR $\left(125 \mathrm{MHz} \mathrm{CDCl}_{3}\right): \delta 11.4,44.9,51.9,73.0,126.5,127.7,128.5,128.5,131.6,136.5,175.7$; IR (neat): 3448, 2365, 1734, 1457, 1200, 968, $750 \mathrm{~cm}^{-1}$; LRMS (ESI): m/z 243 [M+Na] $]^{+}$HRMS (ESI): $\mathrm{m} / \mathrm{z}$ calcd for $\mathrm{C}_{13} \mathrm{H}_{16} \mathrm{O}_{3}[\mathrm{M}+\mathrm{Na}]^{+} 243.0997$ Found 243.0999.

(anti)

$\mathrm{R}_{\mathrm{f}}=0.16(\mathrm{EtOAc} /$ hexane $=1 / 4) ;{ }^{1} \mathrm{H} \mathrm{NMR}\left(500 \mathrm{MHz}, \mathrm{CDCl}_{3}\right): \delta 1.22(\mathrm{~d}, J=7.5 \mathrm{~Hz}, 3 \mathrm{H}), 2.65-2.71$ $(\mathrm{m}, 2 \mathrm{H}), 3.73(\mathrm{~s}, 3 \mathrm{H}), 4.38(\mathrm{q}, J=6.9 \mathrm{~Hz}, 1 \mathrm{H}), 6.18(\mathrm{dd}, J=15.5,6.9 \mathrm{~Hz}, 1 \mathrm{H}), 6.65(\mathrm{~d}, J=15.5 \mathrm{~Hz}$, $1 \mathrm{H}), 7.25-7.27(\mathrm{~m}, 1 \mathrm{H}), 7.32(\mathrm{t}, J=8.0 \mathrm{~Hz}, 2 \mathrm{H}), 7.39(\mathrm{~d}, J=8.0 \mathrm{~Hz}, 2 \mathrm{H}) ;{ }^{13} \mathrm{C} \mathrm{NMR}(125 \mathrm{MHz}$, $\left.\mathrm{CDCl}_{3}\right): \delta 14.2,45.6,51.9,74.7,126.6,127.9,128.6,129.1,132.4,136.3,175.9$; IR (neat): 3446, 2359, 1734, 1457, 1171, 968, $751 \mathrm{~cm}^{-1}$; LRMS (ESI): m/z 243 [M+Na] $]^{+}$HRMS (ESI): m/z calcd for $\mathrm{C}_{13} \mathrm{H}_{16} \mathrm{O}_{3}[\mathrm{M}+\mathrm{Na}]^{+}$243.0997 Found 243.0989.

syn-Methyl 3-hydroxy-2-methyldec-4-ynoate (5ma: methyl ester of 3ma)

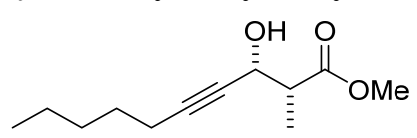

A pale yellow oil; Yield: 51\%; syn/anti =16/1; (ligand: L1, DBU: 2.0 equiv, $\mathrm{C}_{6} \mathrm{H}_{5} \mathrm{CF}_{3}, 12 \mathrm{~h}, \mathrm{GPC}$ ); $\mathrm{R}_{\mathrm{f}}=0.55($ EtOAc/hexane $=1 / 4) ;{ }^{1} \mathrm{H} \mathrm{NMR}\left(500 \mathrm{MHz}, \mathrm{CDCl}_{3}\right): \delta 0.89(\mathrm{t}, J=7.5 \mathrm{~Hz}, 3 \mathrm{H}), 1.24-1.38$ $(\mathrm{m}, 4 \mathrm{H}), 1.29$ (d, $J=7.5 \mathrm{~Hz}, 3 \mathrm{H}), 1.46-1.53(\mathrm{~m}, 2 \mathrm{H}), 2.20(\mathrm{dt}, J=6.9,1.8 \mathrm{~Hz}, 2 \mathrm{H}), 2.74(\mathrm{dq}, J=7.5$, $4.0 \mathrm{~Hz}, 1 \mathrm{H}), 2.87(\mathrm{~d}, J=7.5 \mathrm{~Hz}, \mathrm{OH}), 3.73(\mathrm{~s}, 3 \mathrm{H}), 4.58-4.62(\mathrm{~m}, 1 \mathrm{H}) ;{ }^{13} \mathrm{C} \mathrm{NMR}(125 \mathrm{MHz}$,

\footnotetext{
9 Nagase, R.; Matsumoto, N.; Hosomi, K.; Higashi, T.; Funakoshi, S.; Misaki, T.; Tanabe, Y. Org. Biomol. Chem. 2007, 5, 151.
} 
$\mathrm{CDCl}_{3}$ ): $\delta 11.8,13.9,18.5,22.1,28.2,30.9,45.6,52.0,63.9,78.3,86.6,174.7$; IR (neat): 3469, 2935, $1739,1458,1201,1031 \mathrm{~cm}^{-1}$; LRMS (ESI): $\mathrm{m} / \mathrm{z} 235$ [M+Na] ; HRMS (ESI): $\mathrm{m} / \mathrm{z}$ calcd for $\mathrm{C}_{12} \mathrm{H}_{20} \mathrm{O}_{3}$ $[\mathrm{M}+\mathrm{Na}]^{+} 235.1310$ Found 235.1299.

syn-Methyl 2-(hydroxy(phenyl)methyl)pent-4-enoate (5ab: methyl ester of 3ab)<smiles>COC(=O)[C@H]1CC=C[C@H]1[C@H](O)c1ccccc1</smiles>

A colorless oil; Yield: 80\%; syn/anti $=10 / 1 ;(\mathrm{GPC}) ; \mathrm{R}_{\mathrm{f}}=0.30($ EtOAc/hexane $=1 / 4) ;{ }^{1} \mathrm{H}$ NMR $(500$ $\left.\mathrm{MHz}, \mathrm{CDCl}_{3}\right): \delta 2.36-2.41(\mathrm{~m}, 1 \mathrm{H}), 2.45-2.51(\mathrm{~m}, 1 \mathrm{H}), 2.81-2.85(\mathrm{~m}, 1 \mathrm{H}), 2.86(\mathrm{~d}, J=2.9 \mathrm{~Hz}, 1 \mathrm{H})$, $3.58(\mathrm{~s}, 3 \mathrm{H}), 4.97(\mathrm{~s}, 1 \mathrm{H}), 4.97-5.00(\mathrm{~m}, 1 \mathrm{H}), 5.03(\mathrm{dd}, J=17.2,1.2 \mathrm{~Hz}, 1 \mathrm{H}), 5.69-5.77(\mathrm{~m}, 1 \mathrm{H})$, 7.27-7.30 (m, 1H), $7.34(\mathrm{~d}, J=4.0 \mathrm{~Hz}, 4 \mathrm{H}) ;{ }^{13} \mathrm{C} \mathrm{NMR}\left(125 \mathrm{MHz}, \mathrm{CDCl}_{3}\right): \delta 31.4,51.6,52.7,73.8$, 116.8, 126.1, 127.8, 128.3, 135.3, 141.3, 174.6; IR (neat): 3482, 2951, 1733, 1438, 1168, 1023, 702 $\mathrm{cm}^{-1}$; LRMS (ESI): m/z $243[\mathrm{M}+\mathrm{Na}]^{+}$; HRMS (ESI): m/z calcd for $\mathrm{C}_{13} \mathrm{H}_{16} \mathrm{O}_{3}[\mathrm{M}+\mathrm{Na}]^{+} 243.0997$ Found 243.0986.

syn-Methyl 2-(hydroxy(phenyl)methyl)pent-4-ynoate (5ac: methyl ester of 3ac)<smiles>C#CC[C@H](C(=O)OC)[C@H](O)c1ccccc1</smiles>

A colorless oil; Yield: 92\%; syn/anti $=10 / 1$; (toluene: $2.0 \mathrm{~mL}, \mathrm{GPC}) ; \mathrm{R}_{\mathrm{f}}=0.21(\mathrm{EtOAc} /$ hexane $=$ 1/4); ${ }^{1} \mathrm{H}$ NMR (500 MHz, $\mathrm{CDCl}_{3}$ ): $\delta 1.99$ (t, $\left.J=2.9 \mathrm{~Hz}, 1 \mathrm{H}\right), 2.44-2.49(\mathrm{~m}, 1 \mathrm{H}), 2.64-2.69(\mathrm{~m}, 1 \mathrm{H})$, $2.86(\mathrm{~d}, J=3.5 \mathrm{~Hz}, 1 \mathrm{H}), 2.95-3.00(\mathrm{~m}, 1 \mathrm{H}), 3.64(\mathrm{~s}, 3 \mathrm{H}), 5.05(\mathrm{dd}, J=5.7,3.5 \mathrm{~Hz}, 1 \mathrm{H}), 7.27-7.31$ (m, 1H), 7.33-7.35 (m, 4H); ${ }^{13} \mathrm{C}$ NMR (125 MHz, $\left.\mathrm{CDCl}_{3}\right)$ : $\delta$ 16.7, 51.8, 52.0, 70.1, 73.4, 81.3, 126.0, 128.0, 128.5, 140.7, 173.3; IR (neat): 3482, 3293, 2952, 1732, 1438, 1171, 1024, $703 \mathrm{~cm}^{-1}$; LRMS (ESI): $\mathrm{m} / \mathrm{z} 241[\mathrm{M}+\mathrm{Na}]^{+}$; HRMS (ESI): $\mathrm{m} / \mathrm{z}$ calcd for $\mathrm{C}_{13} \mathrm{H}_{14} \mathrm{O}_{3}[\mathrm{M}+\mathrm{Na}]^{+} 241.0841$ Found 241.0833.

syn-Methyl 2-(hydroxy(phenyl)methyl)-5-(methyl(phenyl)amino)-5-oxopentanoate (5ad: methyl ester of 3ad)<smiles>COC(=O)[C@H](CCC(=O)N(C)O)[C@H](O)c1ccccc1</smiles>

A colorless oil; Yield: 59\%; syn/anti $=6.3 / 1$; (ligand: L2, DBU: 2.5 equiv, solvent: $\mathrm{C}_{6} \mathrm{H}_{5} \mathrm{CF}_{3}, 12 \mathrm{~h}$, neutral $\mathrm{SiO}_{2}$ : EtOAc/hexane $=1 / 4$ to $\left.1 / 1\right) ; \mathrm{R}_{\mathrm{f}}=0.34($ EtOAc/hexane $=1 / 1) ;{ }^{1} \mathrm{H}$ NMR $(500 \mathrm{MHz}$, 
$\left.\mathrm{CDCl}_{3}\right): \delta$ 1.92-2.07 (m, 3H), 2.10-2.20 (m, 1H), 2.69-2.76 (m, 1H), $3.23(\mathrm{~s}, 3 \mathrm{H}), 3.46(\mathrm{~s}, 3 \mathrm{H}), 3.52$ $(\mathrm{d}, J=2.9 \mathrm{~Hz}, \mathrm{OH}), 4.88(\mathrm{brd}, J=5.2 \mathrm{~Hz}, 1 \mathrm{H}), 7.10(\mathrm{~d}, J=7.5 \mathrm{~Hz}, 2 \mathrm{H}), 7.09-7.34(\mathrm{~m}, 6 \mathrm{H})$, 7.36-7.41 (m, 2H); ${ }^{13} \mathrm{C}$ NMR (125 MHz, $\left.\mathrm{CDCl}_{3}\right): \delta 22.7,31.6,37.4,51.3,52.3,73.6,126.2,127.2$, 127.6, 127.8, 128.2, 129.8, 141.6, 143.7, 172.4, 174.6; IR (neat): 3410, 2950, 1733, 1637, 1496, 1163, 1027, $760 \mathrm{~cm}^{-1}$; LRMS (ESI): $\mathrm{m} / \mathrm{z} 364[\mathrm{M}+\mathrm{Na}]^{+}$; HRMS (ESI): $\mathrm{m} / \mathrm{z}$ calcd for $\mathrm{C}_{20} \mathrm{H}_{23} \mathrm{NO}_{4}$ $[\mathrm{M}+\mathrm{Na}]^{+} 364.1525$ Found 364.1513.

syn-Dimethyl 2-(hydroxy(phenyl)methyl)pentanedioate (5ae: methyl ester of 3ae)<smiles>COC(=O)CC[C@H](C(=O)OC)[C@H](O)c1ccccc1</smiles>

A colorless oil; Yield: 91\%; syn/anti $=12 / 1 ; \mathrm{R}_{\mathrm{f}}=0.13($ EtOAc/hexane $=1 / 4) ;{ }^{1} \mathrm{H}$ NMR $(500 \mathrm{MHz}$, $\left.\mathrm{CDCl}_{3}\right): \delta 1.95-2.07(\mathrm{~m}, 2 \mathrm{H}), 2.22-2.29(\mathrm{~m}, 1 \mathrm{H}), 2.33-2.40(\mathrm{~m}, 1 \mathrm{H}), 2.76-2.80(\mathrm{~m}, 1 \mathrm{H}), 2.88(\mathrm{~d}, J=$ $3.4 \mathrm{~Hz}, \mathrm{OH}), 3.60$ (s, 3H), 3.62 (s, 3H), 4.98-5.01 (m, 1H), 7.27-7.29 (m, 1H), 7.34 (d, J = 4.6 Hz, $4 \mathrm{H}) ;{ }^{13} \mathrm{C}$ NMR $\left(125 \mathrm{MHz}, \mathrm{CDCl}_{3}\right): \delta 22.0,31.8,51.6,51.8,51.9,73.8,126.0,127.8,128.4,141.2$, 173.4, 174.6; IR (neat): 3502, 2952, 1736, 1437, 1164, 1025, $703 \mathrm{~cm}^{-1}$; LRMS (ESI): m/z 289 [M+Na] $]^{+}$; HRMS (ESI): $\mathrm{m} / \mathrm{z}$ calcd for $\mathrm{C}_{14} \mathrm{H}_{18} \mathrm{O}_{5}[\mathrm{M}+\mathrm{Na}]^{+} 289.1052$ Found 289.1060 .

syn-Methyl 2-(hydroxy(phenyl)methyl)-7-oxooctanoate (5af: methyl ester of 3af)<smiles>COC(=O)[C@H](CCCC(C)=O)[C@H](O)c1ccccc1</smiles>

A colorless oil; Yield: 48\%; syn/anti $=15 / 1$; (ligand: L2, solvent: $\mathrm{C}_{6} \mathrm{H}_{5} \mathrm{CF}_{3}, 12 \mathrm{~h}, \mathrm{GPC}$ ); $\mathrm{R}_{\mathrm{f}}=0.28$ $($ EtOAc/hexane $=1 / 2) ;{ }^{1} \mathrm{H}$ NMR $\left(500 \mathrm{MHz}, \mathrm{CDCl}_{3}\right): \delta 1.11-1.30(\mathrm{~m}, 2 \mathrm{H}), 1.42-1.64(\mathrm{~m}, 3 \mathrm{H})$, 1.70-1.78 (m, 1H), 2.08 (s, 3H), $2.34(\mathrm{t}, J=7.4 \mathrm{~Hz}, 2 \mathrm{H}), 2.68-2.72(\mathrm{~m}, 1 \mathrm{H}), 2.84$ (s, OH), 3.59 (s, $3 \mathrm{H}), 4.94(\mathrm{~d}, J=5.2 \mathrm{~Hz}, 1 \mathrm{H}), 7.27-7.33(\mathrm{~m}, 5 \mathrm{H}) ;{ }^{13} \mathrm{C} \mathrm{NMR}\left(125 \mathrm{MHz}, \mathrm{CDCl}_{3}\right): \delta 23.4,26.6,27.0$, 29.8, 43.3, 51.6, 52.7, 74.1, 126.0, 127.7, 128.3, 141.5, 175.3, 208.9; IR (neat): 3481, 2950, 1715, 1455, 1362, 1167, 1041, $703 \mathrm{~cm}^{-1}$; LRMS (ESI): m/z $301[\mathrm{M}+\mathrm{Na}]^{+}$; HRMS (ESI): m/z calcd for $\mathrm{C}_{16} \mathrm{H}_{22} \mathrm{O}_{4}[\mathrm{M}+\mathrm{Na}]^{+} 301.1416$ Found 301.1430 .

syn-Methyl 5-chloro-2-(hydroxy(phenyl)methyl)pentanoate (5ag: methyl ester of 3ag)<smiles>COC(=O)[C@H](CCCCl)[C@H](O)c1ccccc1</smiles> 
A colorless oil; Yield: 86\%; syn/anti $=12 / 1 ; \mathrm{R}_{\mathrm{f}}=0.22($ EtOAc/hexane $=1 / 4) ;{ }^{1} \mathrm{H}$ NMR $(500 \mathrm{MHz}$, $\left.\mathrm{CDCl}_{3}\right): \delta 1.62-1.71(\mathrm{~m}, 1 \mathrm{H}), 1.74-1.90(\mathrm{~m}, 3 \mathrm{H}), 2.72-2.76(\mathrm{~m}, 2 \mathrm{H}), 3.46(\mathrm{t}, J=5.8 \mathrm{~Hz}, 2 \mathrm{H}), 3.61(\mathrm{~s}$, $3 \mathrm{H}), 4.98(\mathrm{~d}, J=5.8 \mathrm{~Hz}, 1 \mathrm{H}), 7.28-7.35(\mathrm{~m}, 5 \mathrm{H}) ;{ }^{13} \mathrm{C} \mathrm{NMR}\left(125 \mathrm{MHz}, \mathrm{CDCl}_{3}\right): \delta 24.5,30.6,44.5$, 51.8, 52.3, 74.0, 126.0, 127.9, 128.4, 141.3, 174.9; IR (neat): 3502, 2954, 1733, 1455, 1261, 1026, $702 \mathrm{~cm}^{-1}$; LRMS (ESI): m/z $279[\mathrm{M}+\mathrm{Na}]^{+}$; HRMS (ESI): m/z calcd for $\mathrm{C}_{13} \mathrm{H}_{17} \mathrm{ClO}_{3}[\mathrm{M}+\mathrm{Na}]^{+}$ 279.0764 Found 279.0766.

syn-Methyl 5-(benzylthio)-2-(hydroxy(phenyl)methyl)pentanoate (5ah: methyl ester of 3ah)<smiles>COC(=O)[C@H](CCCSc1ccccc1)[C@@H](O)c1ccccc1</smiles>

A colorless oil; Yield: 93\%; syn/anti $=11 / 1 ; \mathrm{R}_{\mathrm{f}}=0.21($ EtOAc/hexane $=1 / 4) ;{ }^{1} \mathrm{H}$ NMR $(500 \mathrm{MHz}$, $\left.\mathrm{CDCl}_{3}\right): \delta 1.39-1.48(\mathrm{~m}, 1 \mathrm{H}), 1.50-1.56(\mathrm{~m}, 1 \mathrm{H}), 1.67-1.72(\mathrm{~m}, 1 \mathrm{H}), 1.74-1.82(\mathrm{~m}, 1 \mathrm{H}), 2.29-2.38(\mathrm{~m}$, 2H), 2.67-2.71 (m, 1H), $2.74(\mathrm{~d}, J=2.9 \mathrm{~Hz}, \mathrm{OH}), 3.59$ (s, 3H), 3.64 (s, 3H), 4.95 (dd, J= 5.8, $2.9 \mathrm{~Hz}$, 1H), 7.21-7.36 (m, 10H); ${ }^{13} \mathrm{C}$ NMR (125 MHz, $\left.\mathrm{CDCl}_{3}\right): \delta 26.2,27.2,31.0,36.2,51.7,52.5,74.0$, 126.0, 126.9, 127.8, 128.3, 128.4, 128.8, 138.4, 141.4, 175.1; IR (neat): 3446, 2950, 1733, 1454, 1167, 1026, $701 \mathrm{~cm}^{-1}$; LRMS (ESI): m/z $367[\mathrm{M}+\mathrm{Na}]^{+}$; HRMS (ESI): $\mathrm{m} / \mathrm{z}$ calcd for $\mathrm{C}_{20} \mathrm{H}_{24} \mathrm{O}_{3} \mathrm{~S}$ $[\mathrm{M}+\mathrm{Na}]^{+}$367.1344 Found 367.1345.

\section{Methyl 3-hydroxy-2-(methylthio)-3-phenylpropanoate (5ai: methyl ester of 3ai)}<smiles>COC(=O)[C@H](O)[C@H](O)c1ccccc1</smiles>

A colorless oil; Yield: 63\%; syn/anti = 1/1.2; (GPC);

(syn)

$\mathrm{R}_{\mathrm{f}}=0.15($ EtOAc/hexane $=1 / 4) ;{ }^{1} \mathrm{H}$ NMR $\left(500 \mathrm{MHz}, \mathrm{CDCl}_{3}\right): \delta 2.02(\mathrm{~s}, 3 \mathrm{H}), 2.99(\mathrm{~d}, J=5.2 \mathrm{~Hz}$, $1 \mathrm{H}), 3.50(\mathrm{~d}, J=8.6 \mathrm{~Hz}, 1 \mathrm{H}), 3.78(\mathrm{~s}, 3 \mathrm{H}), 4.99(\mathrm{dd}, J=8.6,5.2 \mathrm{~Hz}, 1 \mathrm{H}), 7.32-7.41(\mathrm{~m}, 5 \mathrm{H}) ;{ }^{13} \mathrm{C}$ NMR (125 MHz, $\left.\mathrm{CDCl}_{3}\right): \delta 14.8,52.5,54.0,74.3,126.6,128.5,128.5,140.4,172.1 ;$ IR (neat): 3446, 2361, 1733, 1436, 1154, $700 \mathrm{~cm}^{-1}$; LRMS (ESI): m/z 249 [M+Na] ; HRMS (ESI): m/z calcd for $\mathrm{C}_{11} \mathrm{H}_{14} \mathrm{O}_{3} \mathrm{~S}[\mathrm{M}+\mathrm{Na}]^{+} 249.0561$ Found 249.0571.

(anti)

$\mathrm{R}_{\mathrm{f}}=0.21($ EtOAc/hexane $=1 / 4) ;{ }^{1} \mathrm{H}$ NMR $\left(500 \mathrm{MHz}, \mathrm{CDCl}_{3}\right): \delta 2.20(\mathrm{~s}, 3 \mathrm{H}), 3.30(\mathrm{~d}, J=1.7 \mathrm{~Hz}$, $1 \mathrm{H}), 3.44(\mathrm{~d}, J=8.0 \mathrm{~Hz}, 1 \mathrm{H}), 3.61(\mathrm{~s}, 3 \mathrm{H}), 4.96(\mathrm{~d}, J=8.0 \mathrm{~Hz}, 1 \mathrm{H}), 7.28-7.41(\mathrm{~m}, 5 \mathrm{H}) ;{ }^{13} \mathrm{C} \mathrm{NMR}$ $\left(125 \mathrm{MHz}, \mathrm{CDCl}_{3}\right): \delta=13.7,52.2,55.6,71.1,126.7,128.3,128.4,139.7,170.7$; IR (neat): 3502, 2361, 1733, 1456, 1153, $700 \mathrm{~cm}^{-1}$; LRMS (ESI): m/z 249 [M+Na] ${ }^{+}$; HRMS (ESI): m/z calcd for $\mathrm{C}_{11} \mathrm{H}_{14} \mathrm{O}_{3} \mathrm{~S}[\mathrm{M}+\mathrm{Na}]^{+} 249.0561$ Found 249.0571. 


\section{Methyl 3-hydroxy-3-phenylpropanoate (5aj: methyl ester of 3aj)}<smiles>COC(=O)CC(O)c1ccccc1</smiles>

A colorless oil; Yield: 46\%; (ligand: $\mathbf{L 1}$, solvent: $\mathrm{C}_{6} \mathrm{H}_{5} \mathrm{CF}_{3}, 12 \mathrm{~h}$, neutral $\mathrm{SiO}_{2}$ : EtOAc/hexane =1/5); $\mathrm{R}_{\mathrm{f}}=0.17($ EtOAc/hexane $=1 / 4) ;{ }^{1} \mathrm{H}$ NMR $\left(500 \mathrm{MHz}, \mathrm{CDCl}_{3}\right): \delta 2.72(\mathrm{dd}, J=16.6,3.4 \mathrm{~Hz}, 1 \mathrm{H})$, $2.78(\mathrm{dd}, J=16.6,9.2 \mathrm{~Hz}, 1 \mathrm{H}), 3.20(\mathrm{~s}, \mathrm{OH}), 3.73(\mathrm{~s}, 3 \mathrm{H}), 5.15(\mathrm{dd}, J=9.2,3.4 \mathrm{~Hz}, 1 \mathrm{H}), 7.27-7.32$ (m, 1H), 7.34-7.40 (m, 4H); ${ }^{13} \mathrm{C}$ NMR (125 MHz, $\left.\mathrm{CDCl}_{3}\right): \delta$ 43.1, 51.9, 70.3, 125.6, 127.8, 128.6, 142.4, 172.8; IR (neat): 3468, 3031, 2952, 1737, 1438, 1164, 1025, $701 \mathrm{~cm}^{-1}$; LRMS (ESI): m/z 203 $[\mathrm{M}+\mathrm{Na}]^{+}$; HRMS (ESI): $\mathrm{m} / \mathrm{z}$ calcd for $\mathrm{C}_{10} \mathrm{H}_{12} \mathrm{O}_{3}[\mathrm{M}+\mathrm{Na}]^{+} 203.0684$ Found 203.0677.

Methyl 3-hydroxy-2-methyl-2-(4-((2-oxocyclopentyl)methyl)phenyl)-3-phenylpropanoate (5ak: methyl ester of 3ak)

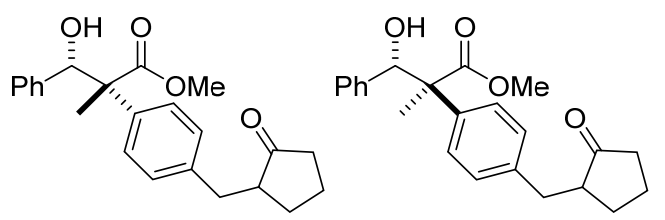

A colorless oil; Yield: 73\%; syn/anti $=2.0 / 1$; (ligand: L1, DBU: 2.5 equiv, solvent: $\mathrm{C}_{6} \mathrm{H}_{5} \mathrm{CF}_{3}, 12 \mathrm{~h}$, GPC);

(syn)

$\mathrm{R}_{\mathrm{f}}=0.17($ EtOAc/hexane $=1 / 4) ;{ }^{1} \mathrm{H}$ NMR $\left(500 \mathrm{MHz}, \mathrm{CDCl}_{3}\right): \delta 1.50(\mathrm{~s}, 3 \mathrm{H}), 1.70-1.80(\mathrm{~m}, 1 \mathrm{H})$, 1.94-2.00 (m, 1H), 2.04-2.14 (m, 2H), 2.33-2.38 (m, 2H), 2.51-2.56 (m, 1H), 3.11 (dd, $J=13.8,3.5$ $\mathrm{Hz}, 1 \mathrm{H}), 3.70(\mathrm{t}, J=3.5 \mathrm{~Hz}, 1 \mathrm{H}), 3.74(\mathrm{~s}, 3 \mathrm{H}), 5.38(\mathrm{~s}, 1 \mathrm{H}), 6.74(\mathrm{~d}, J=7.5 \mathrm{~Hz}, 2 \mathrm{H}), 6.94-7.00(\mathrm{~m}$, $2 \mathrm{H}), 7.04-7.07(\mathrm{~m}, 4 \mathrm{H}), 7.13(\mathrm{t}, J=7.5 \mathrm{~Hz}, 1 \mathrm{H}) ;{ }^{13} \mathrm{C} \mathrm{NMR}\left(125 \mathrm{MHz}, \mathrm{CDCl}_{3}\right): \delta 14.7,14.8,20.6$, $29.0,29.0,35.0,35.0,38.3,50.8,50.9,52.6,56.2,78.5,78.5,126.8,126.8,127.0,127.0,127.2$, 127.6, 128.9, 137.7, 138.4, 138.4, 139.1, 139.2, 177.8, 177.8, 220.1; IR (neat): 3586, 2953, 2360, 1733, 1254, 1042, $704 \mathrm{~cm}^{-1}$; LRMS (ESI): $\mathrm{m} / \mathrm{z} 389[\mathrm{M}+\mathrm{Na}]^{+}$; HRMS (ESI): $\mathrm{m} / \mathrm{z}$ calcd for $\mathrm{C}_{23} \mathrm{H}_{26} \mathrm{O}_{4}$ $[\mathrm{M}+\mathrm{Na}]^{+} 389.1729$ Found 389.1714.

(anti)

$\mathrm{R}_{\mathrm{f}}=0.14($ EtOAc/hexane $=1 / 4) ;{ }^{1} \mathrm{H}$ NMR $\left(500 \mathrm{MHz}, \mathrm{CDCl}_{3}\right): \delta 1.48(\mathrm{~s}, 3 \mathrm{H}), 1.72-1.81(\mathrm{~m}, 1 \mathrm{H})$, 1.96-2.01 (m, 1H), 2.07-2.16 (m, 2H), 2.33-2.37 (m, 2H), 2.51-2.57 (m, 1H), 3.12-3.16 (m, 1H), 3.44 (d, $J=25.2 \mathrm{~Hz}, 1 \mathrm{H}), 3.71(\mathrm{~d}, J=2.9 \mathrm{~Hz}, 3 \mathrm{H}), 5.30(\mathrm{~s}, 1 \mathrm{H}), 6.88(\mathrm{t}, J=8.0 \mathrm{~Hz}, 2 \mathrm{H}), 7.08-7.09(\mathrm{~m}$, 4H), 7.12-7.16 (m, 2H), $7.21(\mathrm{t}, J=7.5 \mathrm{~Hz}, 1 \mathrm{H}) ;{ }^{13} \mathrm{C} \mathrm{NMR}\left(125 \mathrm{MHz}, \mathrm{CDCl}_{3}\right): \delta 20.6,29.1,29.1$, $29.7,35.1,38.2,38.3,50.8,50.9,52.4,52.5,55.5,78.4,127.2$, 127.2, 127.7, 127.7, 128.0, 128.1, 128.3, 139.1, 177.2, 220.3; IR (neat): 3418, 2960, 2359, 1733, 1260, 1032, $799 \mathrm{~cm}^{-1}$; LRMS (ESI): $\mathrm{m} / \mathrm{z} 389[\mathrm{M}+\mathrm{Na}]^{+}$; HRMS (ESI): $\mathrm{m} / \mathrm{z}$ calcd for $\mathrm{C}_{23} \mathrm{H}_{26} \mathrm{O}_{4}[\mathrm{M}+\mathrm{Na}]^{+} 389.1729$ Found 389.1714 . 


\section{Methyl}

2-(1-(4-chlorobenzoyl)-5-methoxy-2-methyl-1H-indol-3-yl)-3-hydroxy-3-phenylpropanoate (5al: methyl ester of 3al)<smiles>COC(=O)C([C@H](C(=O)OC)c1c(C)n(C(=O)c2ccc(Cl)cc2)c2ccc(OC)cc12)[C@@H](O)c1ccc(Cl)cc1</smiles>

A yellow oil; Yield: $63 \%$; syn/anti $=2.4 / 1 ;\left(\mathrm{GPC}\right.$ and $\mathrm{SiO}_{2}$ : EtOAc/hexane $\left.=1 / 2\right)$;

(syn)

$\mathrm{R}_{\mathrm{f}}=0.31($ EtOAc/hexane $=1 / 2) ;{ }^{1} \mathrm{H}$ NMR $\left(500 \mathrm{MHz}, \mathrm{CDCl}_{3}\right): \delta 1.91(\mathrm{~s}, 3 \mathrm{H}), 2.91(\mathrm{~s}, 1 \mathrm{H}), 3.64(\mathrm{~s}$, $3 \mathrm{H}), 3.83(\mathrm{~s}, 3 \mathrm{H}), 4.06(\mathrm{~d}, J=5.7 \mathrm{~Hz}, 1 \mathrm{H}), 5.66(\mathrm{~d}, J=5.7 \mathrm{~Hz}, 1 \mathrm{H}), 6.70(\mathrm{dd}, J=9.2,2.3 \mathrm{~Hz}, 1 \mathrm{H})$, $7.01(\mathrm{~d}, J=8.6 \mathrm{~Hz}, 1 \mathrm{H}), 7.16(\mathrm{~s}, 1 \mathrm{H}), 7.27-7.31(\mathrm{~m}, 5 \mathrm{H}), 7.44(\mathrm{~d}, J=9.2 \mathrm{~Hz}, 2 \mathrm{H}), 7.56$ (d, $J=7.5$ $\mathrm{Hz}, 2 \mathrm{H}) ;{ }^{13} \mathrm{C}$ NMR $\left(125 \mathrm{MHz}, \mathrm{CDCl}_{3}\right): \delta 13.1,50.5,52.2,55.7,73.6,103.0,111.6,112.3,114.6$, 126.6, 127.8, 128.2, 129.1, 129.8, 131.0, 131.3, 133.7, 137.1, 139.4, 141.2, 155.8, 168.2, 173.0; IR (neat): 3524, 2952, 2361, 1683, 1475, 1323, 1090, $755 \mathrm{~cm}^{-1}$; LRMS (ESI): m/z $500[\mathrm{M}+\mathrm{Na}]^{+}$; HRMS (ESI): $\mathrm{m} / \mathrm{z}$ calcd for $\mathrm{C}_{27} \mathrm{H}_{24} \mathrm{ClNO}_{5}[\mathrm{M}+\mathrm{Na}]^{+} 500.1241$ Found 500.1258 .

(anti)

$\mathrm{R}_{\mathrm{f}}=0.22($ EtOAc/hexane $=1 / 2) ;{ }^{1} \mathrm{H}$ NMR $\left(500 \mathrm{MHz}, \mathrm{CDCl}_{3}\right): \delta 1.68(\mathrm{~s}, 3 \mathrm{H}), 3.61(\mathrm{~s}, 1 \mathrm{H}), 3.74(\mathrm{~s}$, $3 \mathrm{H}), 3.87(\mathrm{~s}, 3 \mathrm{H}), 3.97$ (d, $J=9.7 \mathrm{~Hz}, 1 \mathrm{H}), 5.54(\mathrm{~d}, J=9.7 \mathrm{~Hz}, 1 \mathrm{H}), 6.73(\mathrm{dd}, J=9.2,2.9 \mathrm{~Hz}, 1 \mathrm{H})$, 7.07 (d, $J=6.9 \mathrm{~Hz}, 2 \mathrm{H}), 7.12$ (d, $J=8.6 \mathrm{~Hz}, 1 \mathrm{H}), 7.16-7.20$ (m, 3H), 7.22-7.38 (m, 5H); ${ }^{13} \mathrm{C} \mathrm{NMR}$ $\left(125 \mathrm{MHz}, \mathrm{CDCl}_{3}\right): \delta 12.9,51.2,52.5,55.7,73.4,101.9,111.7,113.2,114.8,126.4,127.8,127.9$, 128.9, 129.0, 130.9, 131.1, 133.6, 135.8, 139.4, 140.8, 156.0, 168.1, 174.0; IR (neat): 3566, 2958, 2360, 1684, 1474, 1321, 1038, $757 \mathrm{~cm}^{-1}$; LRMS (ESI): m/z $500[\mathrm{M}+\mathrm{Na}]^{+} ;$HRMS (ESI): m/z calcd for $\mathrm{C}_{27} \mathrm{H}_{24} \mathrm{ClNO}_{5}[\mathrm{M}+\mathrm{Na}]^{+}$500.1241 Found 500.1258.

$(3 R, 5 S, 7 R, 8 R, 10 S, 12 S, 13 R, 14 S, 17 R)-17-\left(\left(2^{\prime} R, 4^{\prime} R, 5 ' R\right)-5 '-H y d r o x y-4 '-(m e t h o x y c a r b o n y l)-5 '-p h\right.$ enylpentan-2'-yl)-10,13-dimethylhexadecahydro-1 $H$-cyclopenta[a]phenanthrene-3,7,12-triyl triacetate (5am: methyl ester of 3am)

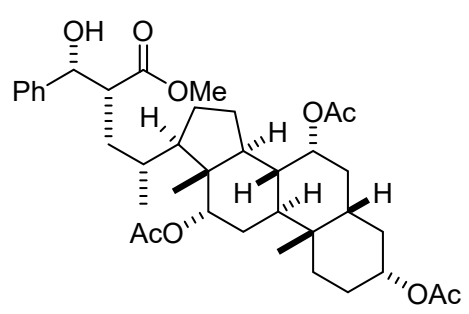

A colorless oil; Yield: 70\%; $\mathrm{dr}=33 / 10 / 4.8 / 1 ;\left(\mathrm{SiO}_{2}\right.$ : EtOAc/hexane $\left.=1 / 4\right) ; \mathrm{R}_{\mathrm{f}}=0.24($ EtOAc/hexane 
$=1 / 2)$

For the most major product: ${ }^{1} \mathrm{H}$ NMR $\left(500 \mathrm{MHz}, \mathrm{CDCl}_{3}\right): \delta 0.68(\mathrm{~s}, 3 \mathrm{H}), 0.70(\mathrm{~d}, J=5.7 \mathrm{~Hz}, 3 \mathrm{H})$, $0.90(\mathrm{~s}, 3 \mathrm{H}), 1.02-1.10(\mathrm{~m}, 2 \mathrm{H}), 1.18-1.51(\mathrm{~m}, 10 \mathrm{H}), 1.59-2.02(\mathrm{~m}, 10 \mathrm{H}), 2.04(\mathrm{~s}, 3 \mathrm{H}), 2.08(\mathrm{~s}, 3 \mathrm{H})$, $2.10(\mathrm{~s}, 3 \mathrm{H}), 2.61(\mathrm{~s}, 1 \mathrm{H}), 2.79-2.83(\mathrm{~m}, 1 \mathrm{H}), 3.57(\mathrm{~s}, 3 \mathrm{H}), 4.54-4.60(\mathrm{~m}, 1 \mathrm{H}), 4.89-4.91(\mathrm{~m}, 2 \mathrm{H})$, $5.04(\mathrm{~s}, 1 \mathrm{H}), 7.28-7.33(\mathrm{~m}, 5 \mathrm{H}) ;{ }^{13} \mathrm{C}$ NMR $\left(125 \mathrm{MHz}, \mathrm{CDCl}_{3}\right): \delta 12.2,17.4,21.4,21.5,21.6,22.6$, 22.8, 25.5, 26.9, 27.1, 28.9, 31.2, 33.2, 33.5, 34.3, 34.6, 34.7, 37.7, 40.9, 43.4, 45.1, 47.9, 50.5, 51.6, 70.7, 74.1, 74.7, 75.3, 126.0, 127.8, 128.3, 141.5, 170.4, 170.5, 175.3; IR (neat): 2959, 2360, 1733, 1541, 1259, 1026, $799 \mathrm{~cm}^{-1}$; LRMS (ESI): m/z $677.6[\mathrm{M}+\mathrm{Na}]^{+}$; HRMS (ESI): $\mathrm{m} / \mathrm{z}$ calcd for $\mathrm{C}_{38} \mathrm{H}_{54} \mathrm{O}_{9}[\mathrm{M}+\mathrm{Na}]^{+}$677.3666 Found 677.3654.

The diastereomers were separated by HPLC with JASCO HPLC systems (pump: PU-2080; detector: UV-2075, measured at $254 \mathrm{~nm}$; column: Shiseido silica (cat. No. 35834); solvent: hexane $/ \mathrm{EtOH}=20 / 1$; flow rate: $1.0 \mathrm{~mL} / \mathrm{min}$; detection: at $254 \mathrm{~nm}$; $\mathrm{rt}$ ): $t_{\mathrm{R}}=65.0 \mathrm{~min}$ (the most major).

The stereochemistry of the most major isomer of $\mathbf{5 a m}$ was determined to be $\left(4^{\prime} R, 5^{\prime} R\right)$ by Mosher ester analysis ${ }^{10}$ (Fig. S4).

To a solution of $5 \mathrm{am}(3.0 \mathrm{mg}, 4.6 \mu \mathrm{mol})$ in $\mathrm{CH}_{2} \mathrm{Cl}_{2}(100 \mu \mathrm{L})$ were added pyridine $(3.7 \mu \mathrm{L}, 46$ $\mu \mathrm{mol})$ and $(R)$-MTPACl $(5.0 \mu \mathrm{L}, 28 \mu \mathrm{mol})$ at room temperature. After stirring for $2 \mathrm{~h}$, the mixture was diluted with EtOAc and quenched with aq. $\mathrm{HCl}(1.0 \mathrm{M})$. The organic layer was separated and the aqueous layer was extracted with EtOAc. The combined organic layer was washed with aq. $\mathrm{HCl}$ $(1.0 \mathrm{M})$, brine, and dried over $\mathrm{Na}_{2} \mathrm{SO}_{4}$. After filtration, the solvent was evaporated under reduced pressure to afford $(S)$-MTPA ester as a colorless oil ( $2.4 \mathrm{mg}, 60 \%$ yield).

$(R)$-MTPA ester was prepared by following the same procedure starting from $\mathbf{5 a m}(4.0 \mathrm{mg}, 6.1$ $\mu \mathrm{mol})$ and $(S)$-MTPACl $(6.6 \mu \mathrm{L}, 37 \mu \mathrm{mol})$ to give $(R)$-MTPA ester as a colorless oil $(2.1 \mathrm{mg}, 40 \%$ yield).

Comparison of the ${ }^{1} \mathrm{H}$ NMR spectra of (S)-MTPA ester and (R)-MTPA ester (in acetone- $d_{6}$ ) indicated that the absolute configuration at $\mathrm{C}^{\prime}$ ' is $(R)$-configuration. Considering that the condition $\mathrm{A}$ generally gives syn-selective products, the stereogenic center at $\mathrm{C}^{\prime}$ ' is assigned as $(R)$-configuration.

${ }^{10}$ Ohtani, I.; Kusumi, T.; Kashman, Y.; Kakisawa, H. J. Am. Chem. Soc. 1991, 113, 4092. 
Figure S4. Mosher ester analysis of the most major isomer of $\mathbf{5 a m}$.

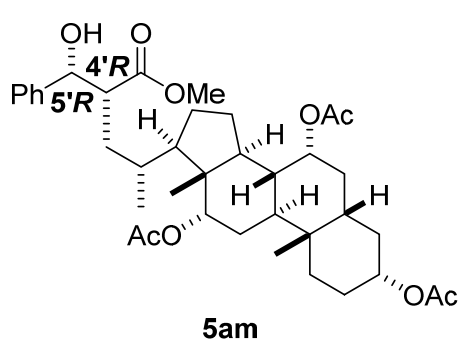

(the most major isomer)

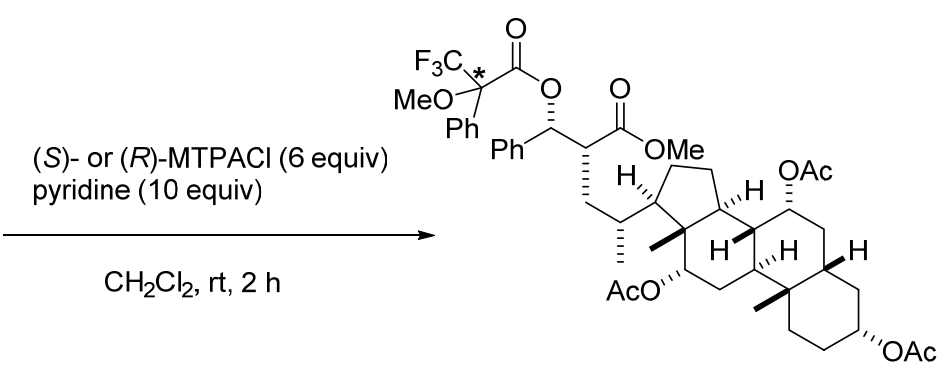

(S)-MTPA ester: $60 \%$ yield (R)-MTPA ester: $40 \%$ yield

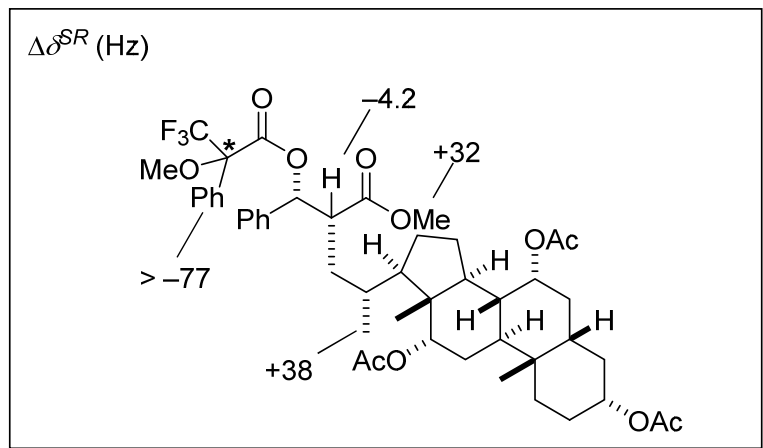



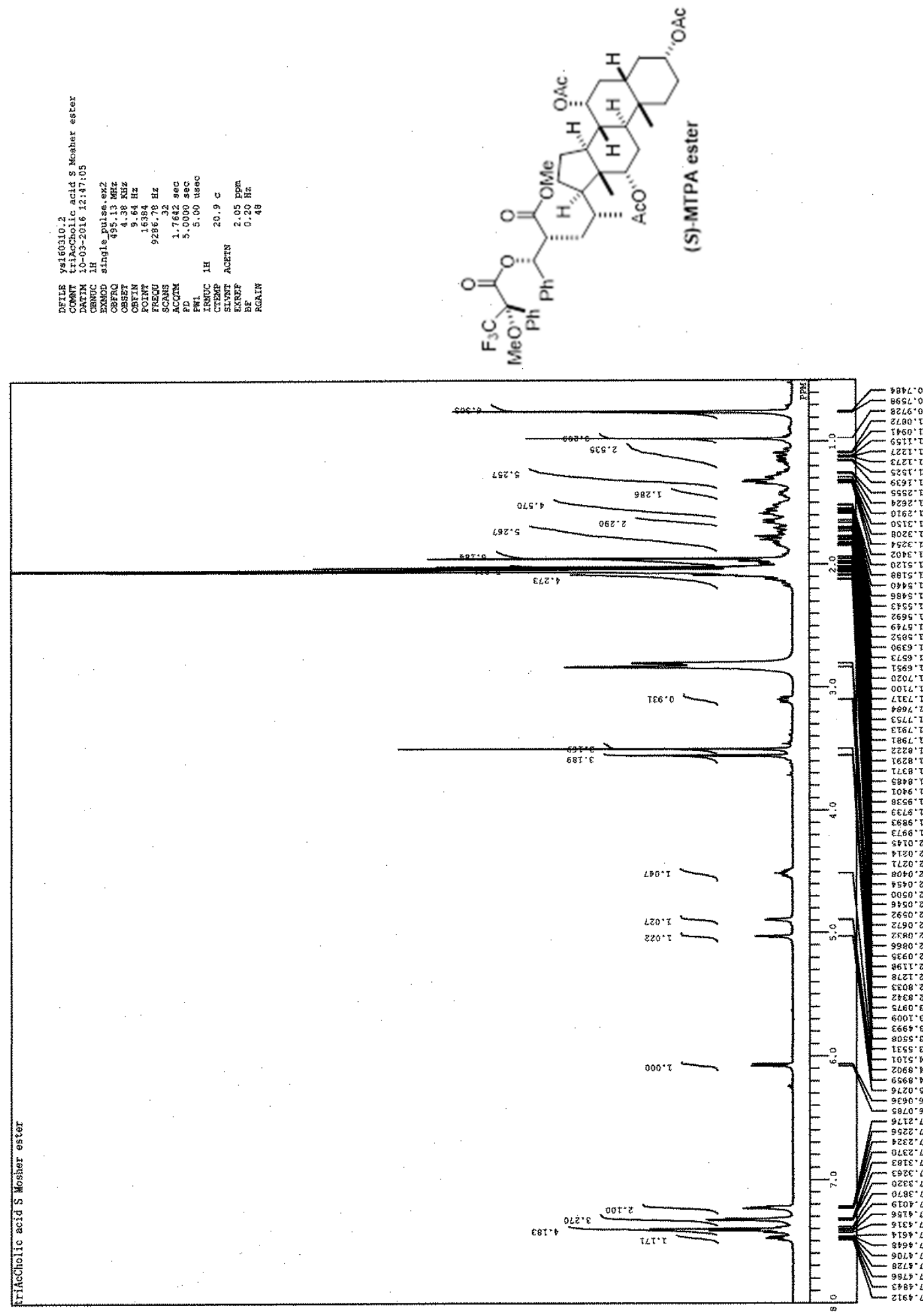

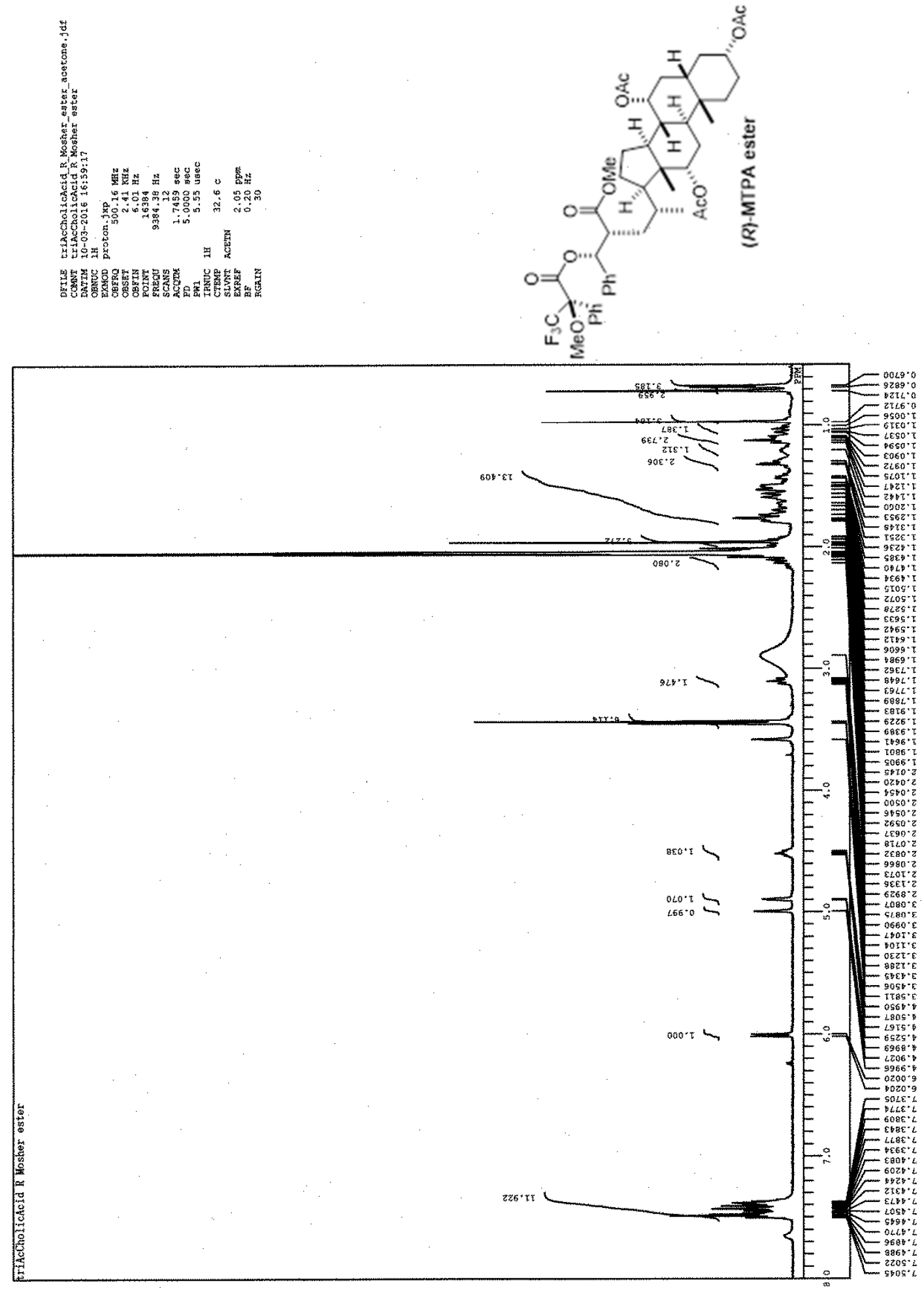
syn-4-Hydroxy-5-(methoxycarbonyl)oct-7-en-1-yl benzoate (5ib: methyl ester of 3ib)<smiles>C=CC[C@H](C(=O)OC)[C@@H](O)CCCOC(=O)c1ccccc1</smiles>

A colorless oil; Yield: 71\%; syn/anti = 7.1/1; (ligand: L2, solvent: $\mathrm{C}_{6} \mathrm{H}_{5} \mathrm{CF}_{3}, 12 \mathrm{~h}, \mathrm{GPC}$ ); $\mathrm{R}_{\mathrm{f}}=0.50$ $($ EtOAc/hexane $=1 / 2) ;{ }^{1} \mathrm{H}$ NMR $\left(500 \mathrm{MHz} \mathrm{CDCl}_{3}\right): \delta 1.58-1.63(\mathrm{~m}, 2 \mathrm{H}), 1.79-1.88(\mathrm{~m}, 1 \mathrm{H})$, 1.98-2.06 (m, 1H), 2.39-2.50 (m, 2H), 2.56-2.62 (m, 2H), $3.68(\mathrm{~s}, 3 \mathrm{H}), 3.88-3.93(\mathrm{~m}, 1 \mathrm{H}), 4.35(\mathrm{t}, J$ $=6.9 \mathrm{~Hz}, 2 \mathrm{H}), 5.02(\mathrm{dd}, J=10.3,1.7 \mathrm{~Hz}, 1 \mathrm{H}), 5.08(\mathrm{dd}, J=16.6,1.7 \mathrm{~Hz}, 1 \mathrm{H}), 5.78(\mathrm{~m}, 1 \mathrm{H}), 7.43(\mathrm{t}$, $J=7.5 \mathrm{~Hz}, 2 \mathrm{H}), 7.55(\mathrm{t}, J=7.5 \mathrm{~Hz}, 1 \mathrm{H}), 8.03(\mathrm{dd}, J=8.0,1.7 \mathrm{~Hz}, 2 \mathrm{H}) ;{ }^{13} \mathrm{C} \mathrm{NMR}(125 \mathrm{MHz}$, $\left.\mathrm{CDCl}_{3}\right): \delta 25.3,30.7,31.6,50.8,51.7,64.7,71.3,116.9,128.3,129.5,130.3,132.9,135.4,166.6$, 174.9; IR (neat): 3506, 2952, 1719, 1451, 1277, 1118, $714 \mathrm{~cm}^{-1} ; \mathrm{LRMS}(\mathrm{ESI}): \mathrm{m} / \mathrm{z} 329[\mathrm{M}+\mathrm{Na}]^{+}$; HRMS (ESI): $\mathrm{m} / \mathrm{z}$ calcd for $\mathrm{C}_{17} \mathrm{H}_{22} \mathrm{O}_{5}[\mathrm{M}+\mathrm{Na}]^{+} 329.1365$ Found 329.1365 .

syn-4-Hydroxy-5-(methoxycarbonyl)oct-7-yn-1-yl benzoate (5ic: methyl ester of 3ic)<smiles>C#CC[C@H](C(=O)OC)[C@@H](O)CCCOC(=O)OCc1ccccc1</smiles>

A white solid; Yield: 73\%; syn/anti $=6.5 / 1$; (ligand: L2, solvent: $\mathrm{C}_{6} \mathrm{H}_{5} \mathrm{CF}_{3}, 12 \mathrm{~h}, \mathrm{GPC}$ ); $\mathrm{R}_{\mathrm{f}}=0.47$ $($ EtOAc/hexane $=1 / 2) ;{ }^{1} \mathrm{H}$ NMR $\left(500 \mathrm{MHz} \mathrm{CDCl}_{3}\right): \delta 1.60-1.66(\mathrm{~m}, 2 \mathrm{H}), 1.83-1.89(\mathrm{~m}, 1 \mathrm{H})$, 1.99-2.04 (m, 2H), 2.54-2.67 (m, 3H), 2.73-2.76 (m, 1H), $3.73(\mathrm{~s}, 3 \mathrm{H}), 4.00-4.03(\mathrm{~m}, 1 \mathrm{H}), 4.36(\mathrm{t}, J$ $=6.9 \mathrm{~Hz}, 2 \mathrm{H}), 7.43(\mathrm{t}, J=7.5 \mathrm{~Hz}, 2 \mathrm{H}), 7.55(\mathrm{t}, J=7.5 \mathrm{~Hz}, 1 \mathrm{H}), 8.03(\mathrm{~d}, J=7.5 \mathrm{~Hz}, 2 \mathrm{H})$; ${ }^{13} \mathrm{C} \mathrm{NMR}$ $\left(125 \mathrm{MHz}_{\mathrm{CDCl}}\right): \delta 16.7,25.3,30.7,49.9,52.1,64.6,70.3,70.9,81.2,128.3,129.5,130.2,132.9$, 166.6, 173.7; IR (KBr): 3500, 3290, 2958, 1725, 1453, 1294, 1107, $717 \mathrm{~cm}^{-1}$; LRMS (ESI): m/z 327 $[\mathrm{M}+\mathrm{Na}]^{+}$; HRMS (ESI): $\mathrm{m} / \mathrm{z}$ calcd for $\mathrm{C}_{17} \mathrm{H}_{20} \mathrm{O}_{5}[\mathrm{M}+\mathrm{Na}]^{+} 327.1208$ Found 327.1197 .

syn-4-Hydroxy-5-(methoxycarbonyl)-8-(methyl(phenyl)amino)-8-oxooctyl benzoate (5id: methyl ester of 3id)<smiles>COC(=O)[C@H](CCC(=O)N(C)c1ccccc1)[C@@H](O)CCCOC(=O)c1ccccc1</smiles>

A colorless oil; Yield: 48\%; syn/anti $=1.6 / 1$; (ligand: L1, solvent: $\mathrm{C}_{6} \mathrm{H}_{5} \mathrm{CF}_{3}, 12 \mathrm{~h}, \mathrm{SiO}_{2}$ : EtOAc/hexane $=1 / 2$ and GPC);

(inseparable diastereomixture)

$\mathrm{R}_{\mathrm{f}}=0.13($ EtOAc/hexane $=1 / 2) ; \operatorname{LRMS}(\mathrm{ESI}): \mathrm{m} / \mathrm{z} 450[\mathrm{M}+\mathrm{Na}]^{+} ; \mathrm{HRMS}(\mathrm{ESI}): \mathrm{m} / \mathrm{z}$ calcd for 
$\mathrm{C}_{24} \mathrm{H}_{29} \mathrm{NO}_{6}[\mathrm{M}+\mathrm{Na}]^{+} 450.1893$ Found 450.1873 .

${ }^{1} \mathrm{H}$ NMR spectral chart of diastereomixture of 5id was shown in Section 7 of this SI.

syn-Methyl 2-(3-(benzoyloxy)propyl)-6-oxotetrahydro-2H-pyran-3-carboxylate (5ie: methyl ester of 3ie)<smiles>COC(=O)[C@H]1CCC(=O)O[C@@H]1CCCOC(=O)OCc1ccccc1</smiles>

A colorless oil; Yield: 54\%; syn/anti = 6.9/1; (ligand: L2, solvent: $\mathrm{C}_{6} \mathrm{H}_{5} \mathrm{CF}_{3}, 12 \mathrm{~h}, \mathrm{GPC}$ );

(inseparable diastereomixture)

$\mathrm{R}_{\mathrm{f}}=0.19($ EtOAc/hexane $=1 / 2) ;$ LRMS (ESI): m/z $343[\mathrm{M}+\mathrm{Na}]^{+} ;$HRMS (ESI): m/z calcd for $\mathrm{C}_{17} \mathrm{H}_{20} \mathrm{O}_{6}[\mathrm{M}+\mathrm{Na}]^{+}$343.1158 Found 343.1163.

${ }^{1} \mathrm{H}$ NMR spectral chart of diastereomixture of 5ie was shown in Section 7 of this SI.

syn-4-Hydroxy-5-(methoxycarbonyl)-10-oxoundecyl benzoate (5if: methyl ester of 3 if)<smiles>COC(=O)[C@H](CCCCC(C)=O)[C@@H](O)CCCOC(=O)c1ccccc1</smiles>

A colorless oil; Yield: 34\%; syn/anti = 3.0/1; (ligand: L1, solvent: $\mathrm{C}_{6} \mathrm{H}_{5} \mathrm{CF}_{3}, 12 \mathrm{~h}, \mathrm{GPC}$ and $\mathrm{SiO}_{2}$ : hexane/EtOAc $=4 / 1)$;

(inseparable diastereomixture)

$\mathrm{R}_{\mathrm{f}}=0.34($ EtOAc/hexane $=1 / 2) ; \operatorname{LRMS}(\mathrm{ESI}): \mathrm{m} / \mathrm{z} 387[\mathrm{M}+\mathrm{Na}]^{+} ;$HRMS $(\mathrm{ESI}): \mathrm{m} / \mathrm{z}$ calcd for $\mathrm{C}_{20} \mathrm{H}_{28} \mathrm{O}_{6}[\mathrm{M}+\mathrm{Na}]^{+}$387.1784 Found 387.1779.

${ }^{1} \mathrm{H}$ NMR spectral chart of diastereomixture of $\mathbf{5}$ if was shown in Section 7 of this SI.

syn-8-Chloro-4-hydroxy-5-(methoxycarbonyl)octyl benzoate (5ig: methyl ester of 3ig)<smiles>[R6]OCCC[C@H](O)[C@@H](CCCCl)C(=O)OC</smiles>

A colorless oil; Yield: 90\%; syn/anti $=11 / 1$; (ligand: L2, solvent: $\mathrm{C}_{6} \mathrm{H}_{5} \mathrm{CF}_{3}, 12 \mathrm{~h}, \mathrm{GPC}$ ); $\mathrm{R}_{\mathrm{f}}=0.49$ $($ EtOAc/hexane $=1 / 2) ;{ }^{1} \mathrm{H}$ NMR $\left(500 \mathrm{MHz} \mathrm{CDCl}_{3}\right): \delta 1.58-1.63(\mathrm{~m}, 2 \mathrm{H}), 1.73-1.86(\mathrm{~m}, 5 \mathrm{H})$, 1.98-2.04 (m, 1H), 2.48-2.51 (m, 1H), $2.57(\mathrm{~s}, 1 \mathrm{H}), 3.53(\mathrm{t}, J=5.8 \mathrm{~Hz}, 2 \mathrm{H}), 3.70(\mathrm{~s}, 3 \mathrm{H}), 3.90(\mathrm{~d}, J=$ $4.0 \mathrm{~Hz}, 1 \mathrm{H}), 4.35(\mathrm{t}, J=6.3 \mathrm{~Hz}, 2 \mathrm{H}), 7.43(\mathrm{t}, J=7.5 \mathrm{~Hz}, 2 \mathrm{H}), 7.54(\mathrm{t}, J=7.5 \mathrm{~Hz}, 1 \mathrm{H}), 8.03(\mathrm{dd}, J=$ 7.5, 1.2 Hz, 2H); ${ }^{13} \mathrm{C}$ NMR (125 MHz, $\left.\mathrm{CDCl}_{3}\right): \delta 24.4,25.3,30.5,30.7,44.5,50.3,51.8,64.6,71.5$, 
128.3, 129.5, 130.2, 132.9, 166.6, 175.2; IR (neat): 3503, 2954, 1718, 1277, 1116, $714 \mathrm{~cm}^{-1}$; LRMS (ESI): $\mathrm{m} / \mathrm{z} 365[\mathrm{M}+\mathrm{Na}]^{+}$; HRMS (ESI): $\mathrm{m} / \mathrm{z}$ calcd for $\mathrm{C}_{17} \mathrm{H}_{23} \mathrm{ClO}_{5}[\mathrm{M}+\mathrm{Na}]^{+} 365.1132$ Found 365.1124 .

syn-8-(Benzylthio)-4-hydroxy-5-(methoxycarbonyl)octyl benzoate (5ih: methyl ester of 3ih)

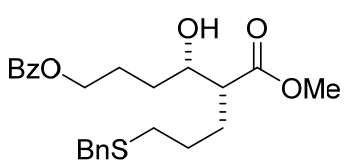

A colorless oil; Yield: 80\%; syn/anti $=8.0 / 1$; (ligand: L2, solvent: $\mathrm{C}_{6} \mathrm{H}_{5} \mathrm{CF}_{3}, 12 \mathrm{~h}, \mathrm{SiO}_{2}$ : EtOAc/hexane $=1 / 9$ to $1 / 4$ and GPC $) ; \mathrm{R}_{\mathrm{f}}=0.16($ EtOAc/hexane $=1 / 4) ;{ }^{1} \mathrm{H}$ NMR $(500 \mathrm{MHz}$, $\left.\mathrm{CDCl}_{3}\right): \delta 1.49-1.62(\mathrm{~m}, 4 \mathrm{H}), 1.67-1.85(\mathrm{~m}, 3 \mathrm{H}), 1.96-2.04(\mathrm{~m}, 1 \mathrm{H}), 2.39-2.46(\mathrm{~m}, 3 \mathrm{H}), 2.51(\mathrm{~d}, J=$ $4.6 \mathrm{~Hz}, \mathrm{OH}), 3.65-3.71(\mathrm{~m}, 5 \mathrm{H}), 3.82-3.87(\mathrm{~m}, 1 \mathrm{H}), 4.35(\mathrm{t}, J=6.9 \mathrm{~Hz}, 2 \mathrm{H}), 7.21-7.24(\mathrm{~m}, 1 \mathrm{H})$, 7.28-7.32 (m, 4H), $7.43(\mathrm{t}, J=7.5 \mathrm{~Hz}, 2 \mathrm{H}), 7.55(\mathrm{dt}, J=7.5,1.2 \mathrm{~Hz}, 1 \mathrm{H}), 8.03(\mathrm{dd}, J=8.1,1.2 \mathrm{~Hz}$, $2 \mathrm{H}) ;{ }^{13} \mathrm{C}$ NMR $\left(125 \mathrm{MHz}, \mathrm{CDCl}_{3}\right): \delta 25.3,26.1,27.1,30.7,31.1,36.2,50.6,51.8,64.6,71.5,126.9$, 128.3, 128.4, 128.8, 129.5, 130.2, 132.9, 138.4, 166.6, 175.4; IR (neat): 3500, 2950, 1717, 1452, 1276, 1118, $713 \mathrm{~cm}^{-1}$; LRMS (ESI): $\mathrm{m} / \mathrm{z} 453[\mathrm{M}+\mathrm{Na}]^{+}$; HRMS (ESI): $\mathrm{m} / \mathrm{z}$ calcd for $\mathrm{C}_{24} \mathrm{H}_{30} \mathrm{O}_{5} \mathrm{~S}$ $[\mathrm{M}+\mathrm{Na}]^{+} 453.1712$ Found 453.1721 .

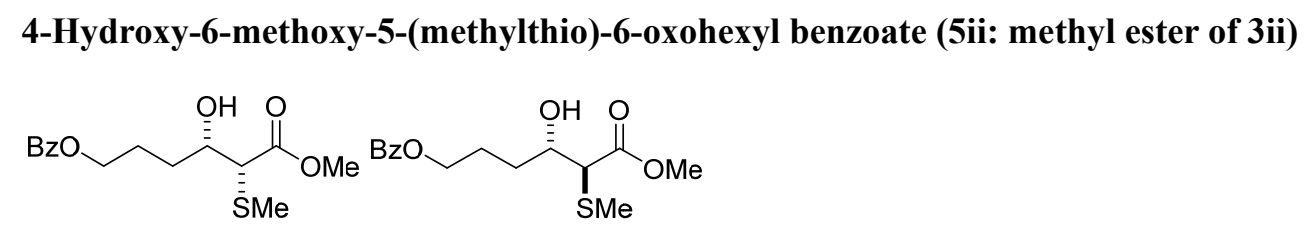

A colorless oil; Yield: 91\%; syn/anti = 1/1.3; (ligand: L2, solvent: $\mathrm{C}_{6} \mathrm{H}_{5} \mathrm{CF}_{3}, 12 \mathrm{~h}, \mathrm{GPC}$ );

(syn)

$\mathrm{R}_{\mathrm{f}}=0.28($ EtOAc/hexane $=1 / 2) ;{ }^{1} \mathrm{H}$ NMR $\left(500 \mathrm{MHz}, \mathrm{CDCl}_{3}\right): \delta 1.69-1.73(\mathrm{~m}, 2 \mathrm{H}), 1.85-1.92(\mathrm{~m}$, 1H), 2.00-2.06 (m, 1H), 2.19 (s, 3H), $3.04(\mathrm{~s}, 1 \mathrm{H}), 3.16$ (d, $J=6.9 \mathrm{~Hz}, 1 \mathrm{H}), 3.74(\mathrm{~s}, 3 \mathrm{H}), 3.96-4.00$ $(\mathrm{m}, 1 \mathrm{H}), 4.36(\mathrm{t}, J=6.3 \mathrm{~Hz}, 2 \mathrm{H}), 7.43(\mathrm{t}, J=7.5 \mathrm{~Hz}, 2 \mathrm{H}), 7.55$ (t, $J=7.5 \mathrm{~Hz}, 1 \mathrm{H}), 8.03$ (dd, $J=8.6$, $1.1 \mathrm{~Hz}, 2 \mathrm{H}) ;{ }^{13} \mathrm{C} \mathrm{NMR}\left(125 \mathrm{MHz}, \mathrm{CDCl}_{3}\right): \delta 14.1,25.1,30.6,52.3,53.7,64.6,68.6,128.3,129.5$, 130.3, 132.9, 166.6, 171.7; IR (neat): 3503, 2952, 1718, 1277, 1118, $714 \mathrm{~cm}^{-1}$; LRMS (ESI): m/z $335[\mathrm{M}+\mathrm{Na}]^{+}$; HRMS (ESI): $\mathrm{m} / \mathrm{z}$ calcd for $\mathrm{C}_{15} \mathrm{H}_{20} \mathrm{O}_{5} \mathrm{~S}[\mathrm{M}+\mathrm{Na}]^{+} 335.0929$ Found 335.0931.

(anti)

$\mathrm{R}_{\mathrm{f}}=0.25($ EtOAc/hexane $=1 / 2) ;{ }^{1} \mathrm{H}$ NMR $\left(500 \mathrm{MHz}, \mathrm{CDCl}_{3}\right): \delta 1.59-1.69(\mathrm{~m}, 1 \mathrm{H}), 1.85-1.92(\mathrm{~m}$, $1 \mathrm{H}), 1.99-2.06(\mathrm{~m}, 2 \mathrm{H}), 2.15(\mathrm{~s}, 3 \mathrm{H}), 2.73-2.77(\mathrm{~m}, 1 \mathrm{H}), 3.21$ (d, $J=8.0 \mathrm{~Hz}, 1 \mathrm{H}), 3.78(\mathrm{~s}, 3 \mathrm{H})$, 3.97-4.02 (m, 1H), $4.37(\mathrm{t}, J=6.3 \mathrm{~Hz}, 2 \mathrm{H}), 7.43(\mathrm{t}, J=7.5 \mathrm{~Hz}, 2 \mathrm{H}), 7.55(\mathrm{t}, J=7.5 \mathrm{~Hz}, 1 \mathrm{H}), 8.04$ $(\mathrm{dd}, J=7.5,1.2 \mathrm{~Hz}, 2 \mathrm{H}) ;{ }^{13} \mathrm{C} \mathrm{NMR}\left(125 \mathrm{MHz}, \mathrm{CDCl}_{3}\right): \delta 14.4,25.0,30.5,52.4,52.5,64.6,70.6$, 128.3, 129.5, 130.3, 132.9, 166.6, 172.2; IR (neat): 3482, 2952, 1717, 1276, 1119, $713 \mathrm{~cm}^{-1}$; LRMS 
(ESI): $\mathrm{m} / \mathrm{z} 335[\mathrm{M}+\mathrm{Na}]^{+}$; HRMS (ESI): $\mathrm{m} / \mathrm{z}$ calcd for $\mathrm{C}_{15} \mathrm{H}_{20} \mathrm{O}_{5} \mathrm{~S}[\mathrm{M}+\mathrm{Na}]^{+} 335.0929$ Found 335.0931 .

4-Hydroxy-6-methoxy-6-oxohexyl benzoate (5ij: methyl ester of 3ij)<smiles>COC(=O)CCCC(O)CC(=O)OC</smiles>

A colorless oil; Yield: 28\%; (ligand: $\mathbf{L 1}$, solvent: $\mathrm{C}_{6} \mathrm{H}_{5} \mathrm{CF}_{3}, 12 \mathrm{~h}, \mathrm{GPC}$ and $\mathrm{SiO}_{2}$ : EtOAc/hexane $=$ $1 / 2) ; \mathrm{R}_{\mathrm{f}}=0.25($ EtOAc/hexane $=1 / 2) ;{ }^{1} \mathrm{H}$ NMR $\left(500 \mathrm{MHz}, \mathrm{CDCl}_{3}\right): \delta 1.58-1.70(\mathrm{~m}, 2 \mathrm{H}), 1.82-1.90$ (m, 1H), 1.94-2.02 (m, 1H), 2.43-2.56 (m, 2H), $3.06(\mathrm{~s}, 1 \mathrm{H}), 3.71(\mathrm{~s}, 3 \mathrm{H}), 4.07-4.09(\mathrm{~m}, 1 \mathrm{H}), 4.36$ (dt, $J=6.3,2.3 \mathrm{~Hz}, 2 \mathrm{H}), 7.43(\mathrm{t}, J=8.1 \mathrm{~Hz}, 2 \mathrm{H}), 7.55(\mathrm{t}, J=7.5 \mathrm{~Hz}, 1 \mathrm{H}), 8.03(\mathrm{~d}, J=6.9 \mathrm{~Hz}, 2 \mathrm{H})$; ${ }^{13} \mathrm{C}$ NMR (125 MHz, $\left.\mathrm{CDCl}_{3}\right): \delta 24.9,32.9,41.1,51.8,64.7,67.5,128.3,129.5,130.3,132.9,166.6$, 173.3; IR (neat): 3503, 2953, 1717, 1451, 1277, 1118, $714 \mathrm{~cm}^{-1}$; LRMS (ESI): m/z 289 [M+Na] ; HRMS (ESI): $\mathrm{m} / \mathrm{z}$ calcd for $\mathrm{C}_{14} \mathrm{H}_{18} \mathrm{O}_{5}[\mathrm{M}+\mathrm{Na}]^{+} 289.1052$ Found 289.1053 .

4-Hydroxy-6-methoxy-5-methyl-6-0xo-5-(4-((2-oxocyclopentyl)methyl)phenyl)hexyl benzoate (5ik: methyl ester of 3ik)

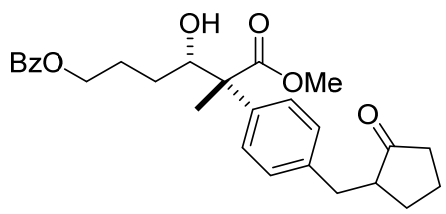

A colorless oil; Yield: $56 \%$; syn/anti $=3.1 / 1$; (ligand: $\mathbf{L 1}$, solvent: $\mathrm{C}_{6} \mathrm{H}_{5} \mathrm{CF}_{3}, 12 \mathrm{~h}, \mathrm{SiO}_{2}$ : EtOAc/hexane $=1 / 2$ and GPC);

\section{(inseparable diastereomixture)}

$\mathrm{R}_{\mathrm{f}}=0.24($ EtOAc/hexane $=1 / 2)$; LRMS (ESI): m/z $475[\mathrm{M}+\mathrm{Na}]^{+}$; HRMS (ESI): m/z calcd for $\mathrm{C}_{27} \mathrm{H}_{32} \mathrm{O}_{6}[\mathrm{M}+\mathrm{Na}]^{+}$475.2097 Found 475.2086.

${ }^{1} \mathrm{H}$ NMR spectral chart of diastereomixture of $\mathbf{5 i k}$ was shown in Section 7 of this SI.

5-(1-(4-Chlorobenzoyl)-5-methoxy-2-methyl-1H-indol-3-yl)-4-hydroxy-6-methoxy-6-oxohexyl benzoateate (5il: methyl ester of 3il)
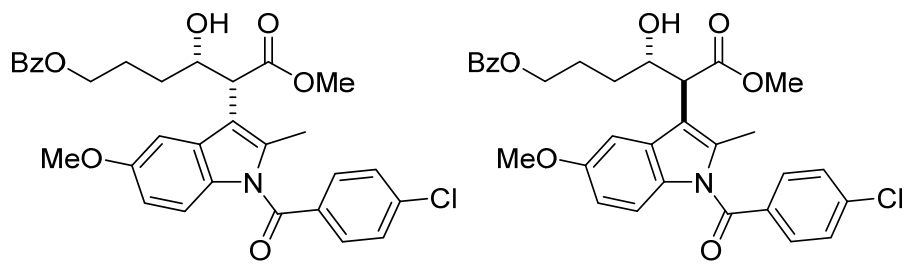

A yellow oil; Yield: 40\%; syn/anti $=2.0 / 1$; (ligand: L1, solvent: $\mathrm{C}_{6} \mathrm{H}_{5} \mathrm{CF}_{3}, 12 \mathrm{~h}, \mathrm{SiO}_{2}$ : EtOAc/hexane $=1 / 2$ and $\mathrm{GPC})$;

(syn) 
$\mathrm{R}_{\mathrm{f}}=0.22($ EtOAc/hexane $=1 / 2) ;{ }^{1} \mathrm{H}$ NMR $\left(500 \mathrm{MHz}, \mathrm{CDCl}_{3}\right): \delta 1.43-1.50(\mathrm{~m}, 2 \mathrm{H}), 1.77-1.83(\mathrm{~m}$, $1 \mathrm{H}), 1.97-2.06(\mathrm{~m}, 1 \mathrm{H}), 2.40$ (s, 3H), 3.39 (d, $J=3.5 \mathrm{~Hz}, 1 \mathrm{H}), 3.69$ (s, 3H), 3.79 (s, 3H), 3.83 (d, $J=$ $10.3 \mathrm{~Hz}, 1 \mathrm{H}), 4.27$ (t, $J=6.9 \mathrm{~Hz}, 2 \mathrm{H}), 4.59-4.64(\mathrm{~m}, 1 \mathrm{H}), 6.64$ (dd, $J=8.6,2.9 \mathrm{~Hz}, 1 \mathrm{H}), 6.81$ (d, $J=$ $9.2 \mathrm{~Hz}, 1 \mathrm{H}), 7.01(\mathrm{~d}, J=2.3 \mathrm{~Hz}, 1 \mathrm{H}), 7.35(\mathrm{t}, J=8.1 \mathrm{~Hz}, 2 \mathrm{H}), 7.42(\mathrm{~d}, J=8.6 \mathrm{~Hz}, 2 \mathrm{H}), 7.50(\mathrm{t}, J=$ $8.6 \mathrm{~Hz}, 1 \mathrm{H}), 7.60(\mathrm{~d}, J=8.0 \mathrm{~Hz}, 2 \mathrm{H}), 7.85(\mathrm{~d}, J=8.0 \mathrm{~Hz}, 2 \mathrm{H}) ;{ }^{13} \mathrm{C}$ NMR $\left(125 \mathrm{MHz}, \mathrm{CDCl}_{3}\right): \delta 13.4$, 24.8, 29.9, 49.6, 52.4, 55.6, 64.4, 70.0, 101.8, 111.7, 114.0, 114.9, 128.3, 129.0, 129.2, 129.4, 130.2, 130.8, 131.2, 132.8, 133.6, 136.1, 139.5, 155.9, 166.5, 168.2, 175.0; IR (neat): 3508, 2952, 1717, 1476, 1276, 1090, $756 \mathrm{~cm}^{-1}$; LRMS (ESI): m/z $586.5[\mathrm{M}+\mathrm{Na}]^{+}$; HRMS (ESI): m/z calcd for $\mathrm{C}_{31} \mathrm{H}_{30} \mathrm{ClNO}_{7}[\mathrm{M}+\mathrm{Na}]^{+}$586.1608 Found 586.1614.

(anti)

$\mathrm{R}_{\mathrm{f}}=0.37($ EtOAc/hexane $=1 / 2) ;{ }^{1} \mathrm{H}$ NMR $\left(500 \mathrm{MHz}, \mathrm{CDCl}_{3}\right): \delta 1.60-1.66(\mathrm{~m}, 1 \mathrm{H}), 1.75-1.80(\mathrm{~m}$, 1H), 1.89-1.96 (m, 1H), 2.04-2.13 (m, 1H), 2.35 (s, 1H), $2.40(\mathrm{~s}, 3 \mathrm{H}), 3.68(\mathrm{~s}, 3 \mathrm{H}), 3.79(\mathrm{~s}, 3 \mathrm{H}), 3.87$ (d, $J=6.9 \mathrm{~Hz}, 1 \mathrm{H}), 4.34-4.42(\mathrm{~m}, 2 \mathrm{H}), 4.53(\mathrm{~s}, 1 \mathrm{H}), 6.67(\mathrm{dd}, J=9.2,2.9 \mathrm{~Hz}, 1 \mathrm{H}), 6.85(\mathrm{~d}, J=9.2$ $\mathrm{Hz}, 1 \mathrm{H}), 7.14(\mathrm{~d}, J=2.3 \mathrm{~Hz}, 1 \mathrm{H}), 7.41-7.47(\mathrm{~m}, 4 \mathrm{H}), 7.55(\mathrm{t}, J=7.5 \mathrm{~Hz}, 1 \mathrm{H}), 7.66(\mathrm{~d}, J=8.6 \mathrm{~Hz}$, $2 \mathrm{H}), 8.01(\mathrm{~d}, J=7.5 \mathrm{~Hz}, 2 \mathrm{H}) ;{ }^{13} \mathrm{C} \mathrm{NMR}\left(125 \mathrm{MHz}, \mathrm{CDCl}_{3}\right): \delta 13.6,25.3,31.2,48.9,52.2,55.6,64.7$, 70.6, 102.6, 111.8, 113.0, 114.8, 128.3, 129.2, 129.4, 129.5, 130.3, 131.0, 131.3, 132.9, 133.6, 137.2, 139.6, 155.9, 166.6, 168.3, 172.9; IR (neat): 3510, 2955, 1717, 1476, 1276, 1090, $756 \mathrm{~cm}^{-1}$; LRMS (ESI): $\mathrm{m} / \mathrm{z} 586.5[\mathrm{M}+\mathrm{Na}]^{+}$; HRMS (ESI): $\mathrm{m} / \mathrm{z}$ calcd for $\mathrm{C}_{31} \mathrm{H}_{30} \mathrm{ClNO}_{7}[\mathrm{M}+\mathrm{Na}]^{+} 586.1608$ Found 586.1612 .

(3R,5S,7R,8R,9S,10S,12S,13R,14S,17R)-17-((2'R,4'R,5'S)-8'-(Benzoyloxy)-5'-hydroxy-4'-(meth oxycarbonyl)octan-2'-yl)-10,13-dimethylhexadecahydro- $1 H$-cyclopenta[a]phenanthrene-3,7,12triyl triacetate (5im: methyl ester of 3im)

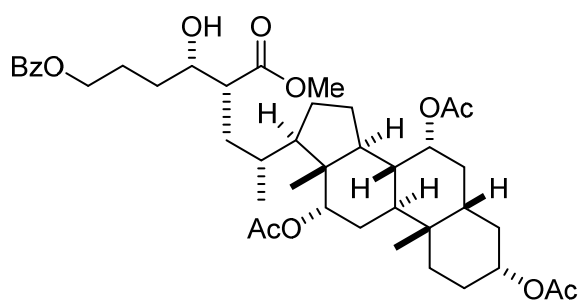

A white solid; Yield: $63 \%$; $\mathrm{dr}=14 / 4.1 / 1.2 / 1$; (ligand: L2, solvent: $\mathrm{C}_{6} \mathrm{H}_{5} \mathrm{CF}_{3}, 12 \mathrm{~h}, \mathrm{SiO}_{2}$ : EtOAc/hexane $=1 / 2$ and GPC);

(inseparable diastereomixture)

$\mathrm{R}_{\mathrm{f}}=0.19\left(\right.$ EtOAc/hexane = 1/2); LRMS (ESI): m/z $763.6[\mathrm{M}+\mathrm{Na}]^{+}$; HRMS (ESI): $\mathrm{m} / \mathrm{z}$ calcd for $\mathrm{C}_{42} \mathrm{H}_{60} \mathrm{O}_{11}[\mathrm{M}+\mathrm{Na}]^{+} 763.4033$ Found 763.4056 .

${ }^{1} \mathrm{H}$ NMR spectral chart of diastereomixture of $5 \mathbf{i m}$ was shown in Section 7 of this SI. 
Methyl 9-hydroxy-7-oxo-9-phenylnonanoate (6: methyl ester of 4)

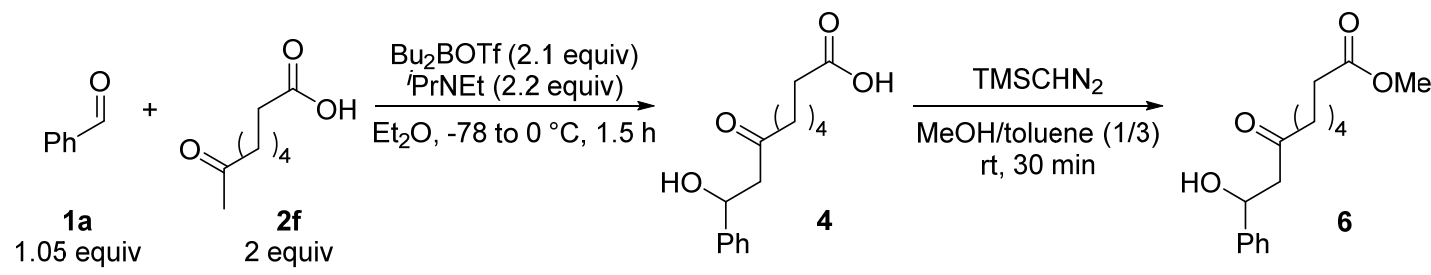

A flame-dried $20 \mathrm{~mL}$ test tube, equipped with a magnetic stirring bar, was charged with ${ }^{i} \mathrm{Pr}_{2} \mathrm{NEt}$ $(115.1 \mu \mathrm{L}, 0.66 \mathrm{mmol})$ and anhydrous $\mathrm{Et}_{2} \mathrm{O}(2.3 \mathrm{~mL})$, before $\mathrm{Bu}_{2} \mathrm{BOTf}\left(1.0 \mathrm{M}\right.$ in $\mathrm{CH}_{2} \mathrm{Cl}_{2}, 630 \mu \mathrm{L}$, $0.63 \mathrm{mmol}$ ) was added at $0{ }^{\circ} \mathrm{C}$. After stirring for $45 \mathrm{~min}$ and cooled to $-78{ }^{\circ} \mathrm{C}$, benzaldehyde (1a) $(32$ $\mu \mathrm{L}, 0.32 \mathrm{mmol}$ ) was added. The reaction mixture was stirred for $30 \mathrm{~min}$ at $-78{ }^{\circ} \mathrm{C}$ and $1 \mathrm{~h}$ at $0{ }^{\circ} \mathrm{C}$. Subsequently, the reaction was quenched with sat. aq. $\mathrm{NaHCO}_{3}$ and the aqueous layer was separated. The organic layer was extracted with sat. aq. $\mathrm{NaHCO}_{3}$. The combined aqueous layer was successively acidified to $\mathrm{pH} 2$ with aq. $\mathrm{HCl}(6.0 \mathrm{M})$, extracted with $\mathrm{Et}_{2} \mathrm{O}$, dried over $\mathrm{Na}_{2} \mathrm{SO}_{4}$, filtered and concentrated under reduced pressure to afford the crude product 4 .

Subsequent methyl esterification was carried out without further purification. To a solution of

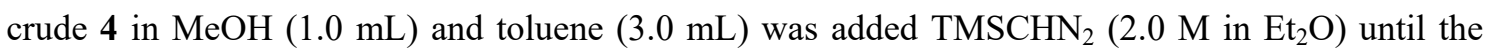
color of the solution became yellow. After stirring for $30 \mathrm{~min}$ at room temperature, a few drops of acetic acid were added until the yellow color of the solution disappeared. The solvent was evaporated under reduced pressure and the remaining residue was purified by silica gel column chromatography $($ EtOAc/hexane $=1 / 9)$, affording the product 6 as a colorless oil $(32 \mathrm{mg}, 39 \%$ yield).

A colorless oil; Yield: 39\%; $\mathrm{R}_{\mathrm{f}}=0.38($ EtOAc/hexane $=1 / 2) ;{ }^{1} \mathrm{H}$ NMR $\left(500 \mathrm{MHz}, \mathrm{CDCl}_{3}\right): \delta$ 1.25-1.34 (m, 2H), 1.57-1.65 (m, 4H), 2.30 (t, $J=7.5 \mathrm{~Hz}, 2 \mathrm{H}), 2.44(\mathrm{t}, J=7.5 \mathrm{~Hz}, 2 \mathrm{H}), 2.76-2.88(\mathrm{~m}$, 2H), $3.32(\mathrm{~s}, \mathrm{OH}), 3.66(\mathrm{~s}, 3 \mathrm{H}), 5.15(\mathrm{dd}, J=9.2,2.9 \mathrm{~Hz}, 1 \mathrm{H}), 7.27-7.36(\mathrm{~m}, 5 \mathrm{H}) ;{ }^{13} \mathrm{C}$ NMR $(125$ $\left.\mathrm{MHz}, \mathrm{CDCl}_{3}\right): \delta 23.0,24.6,28.5,33.8,43.3,51.0,51.5,69.9,125.6,127.6,128.5,142.8,174.0$, 211.2; IR (neat): 3463, 2937, 1736, 1714, 1495, 1364, 1173, $702 \mathrm{~cm}^{-1}$; LRMS (ESI): $\mathrm{m} / \mathrm{z} 301$ $[\mathrm{M}+\mathrm{Na}]^{+}$; HRMS (ESI): $\mathrm{m} / \mathrm{z}$ calcd for $\mathrm{C}_{16} \mathrm{H}_{22} \mathrm{O}_{4}[\mathrm{M}+\mathrm{Na}]^{+}$301.1416 Found 301.1430. 


\section{NMR Charts}
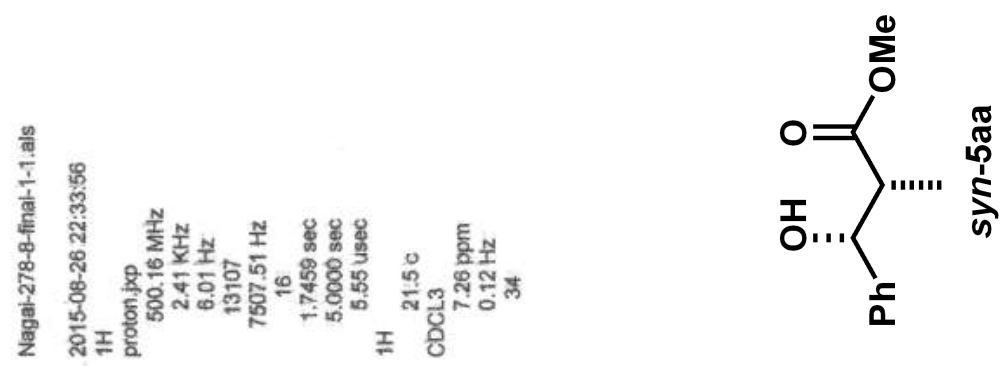

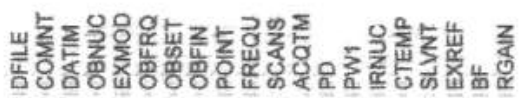

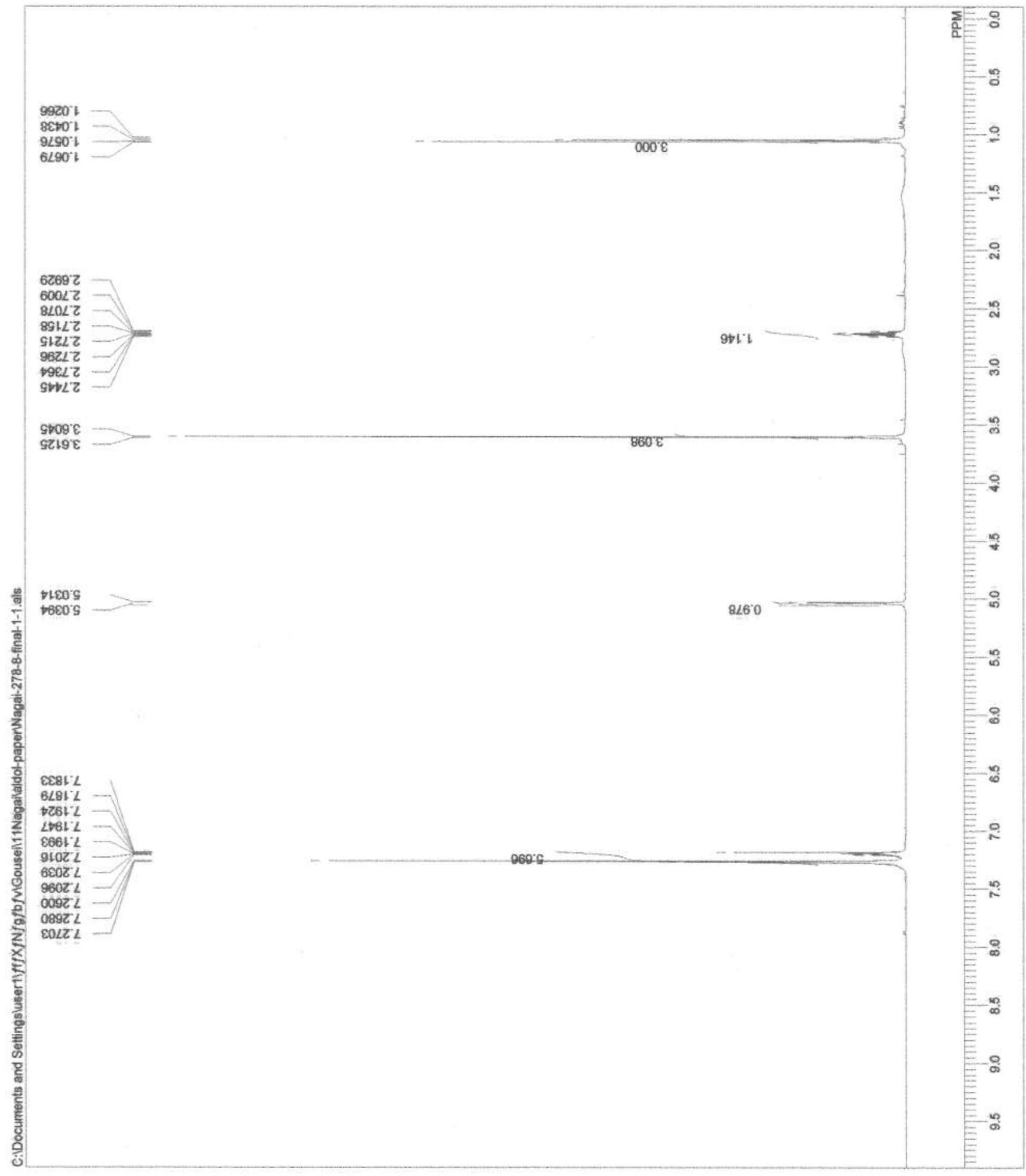




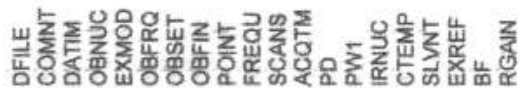

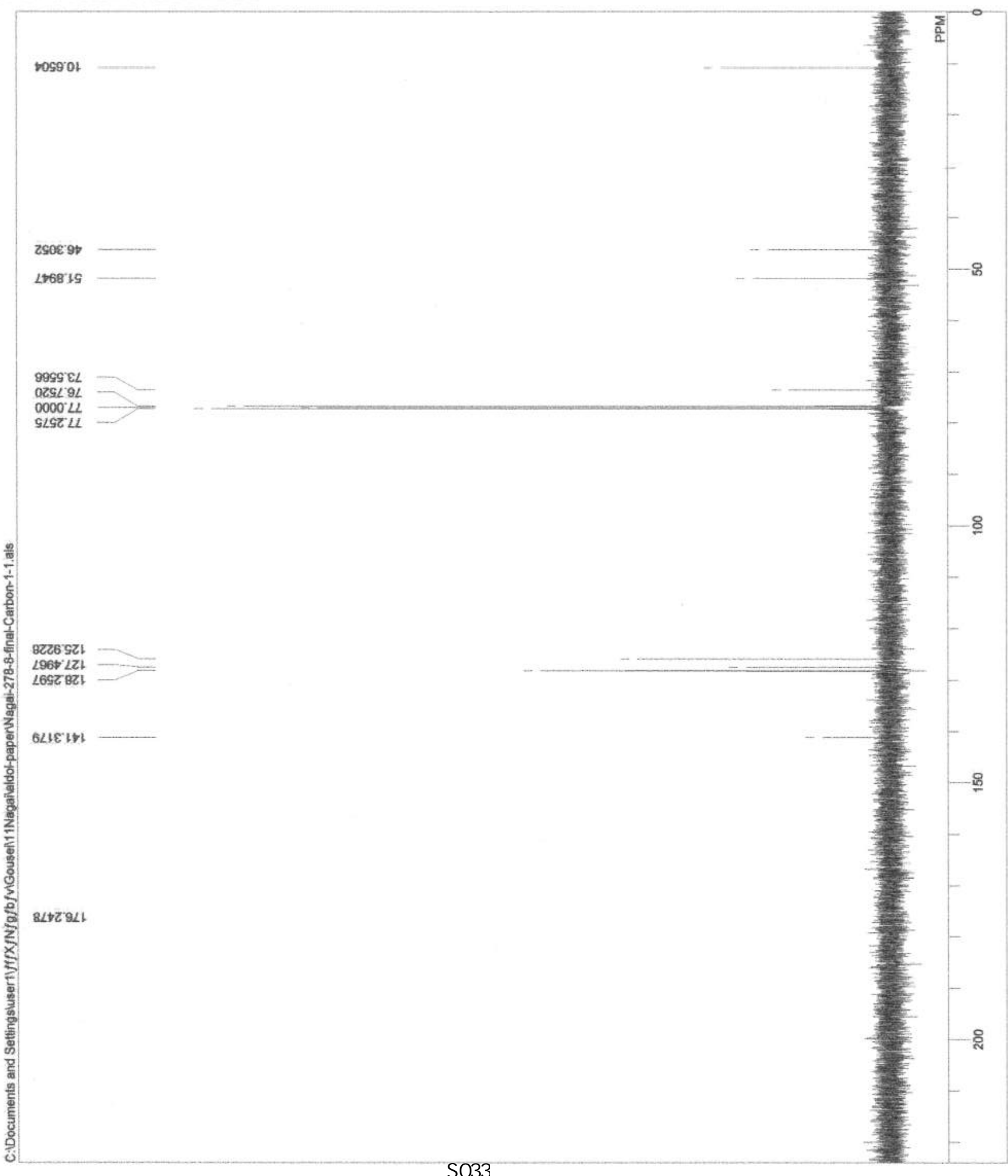




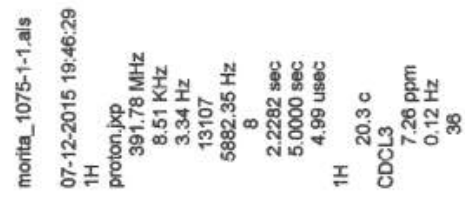

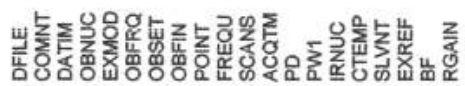

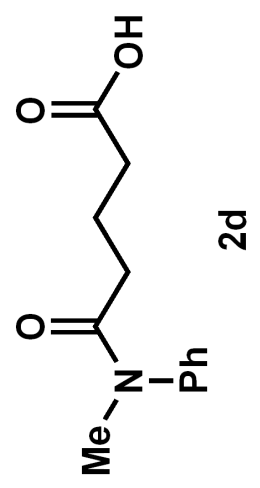

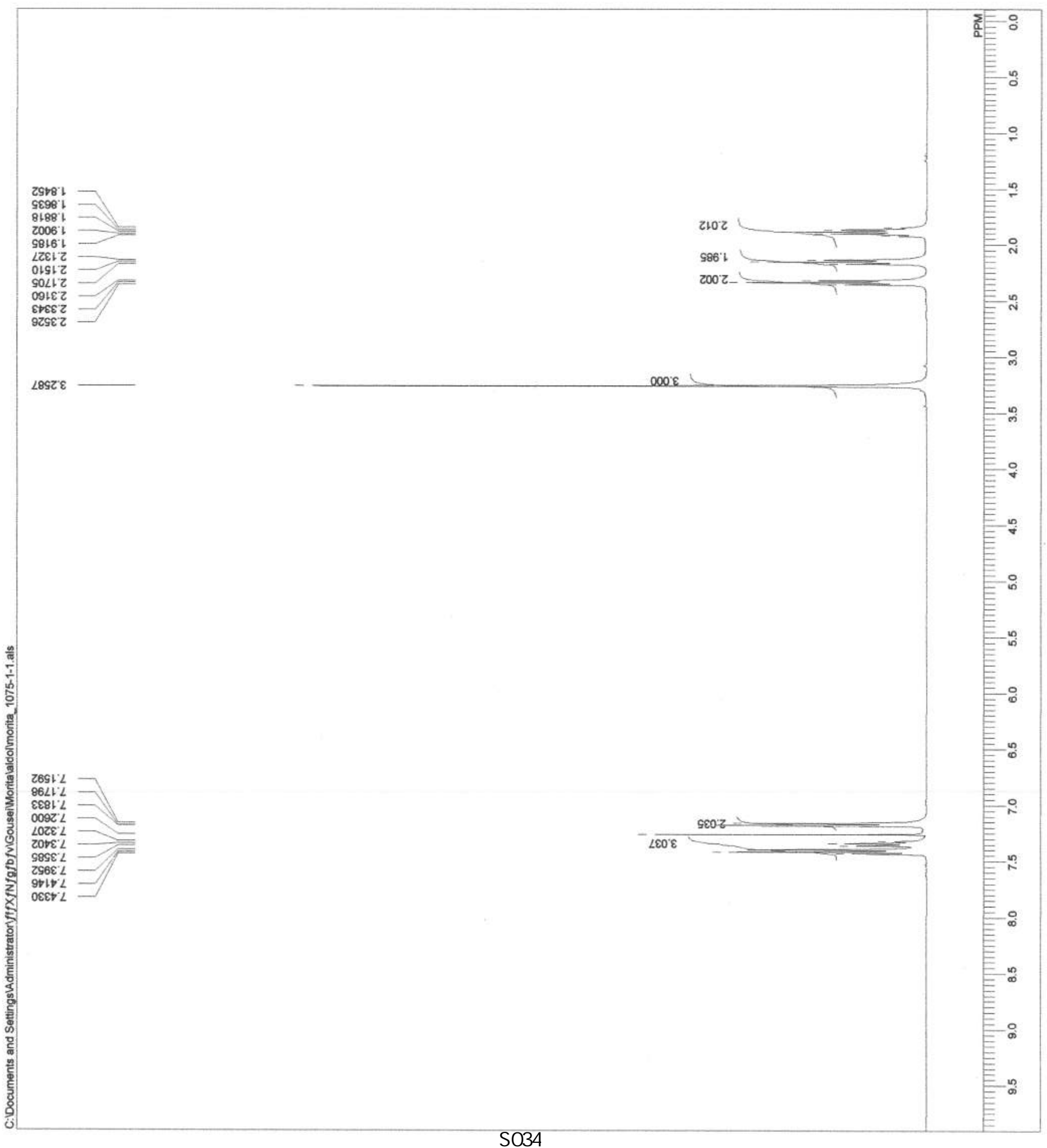



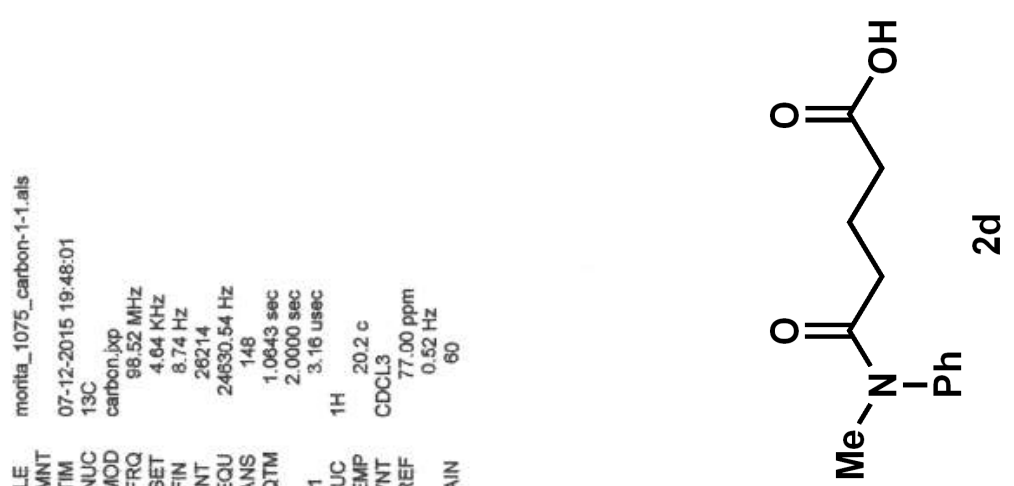

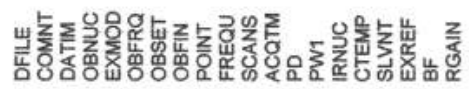

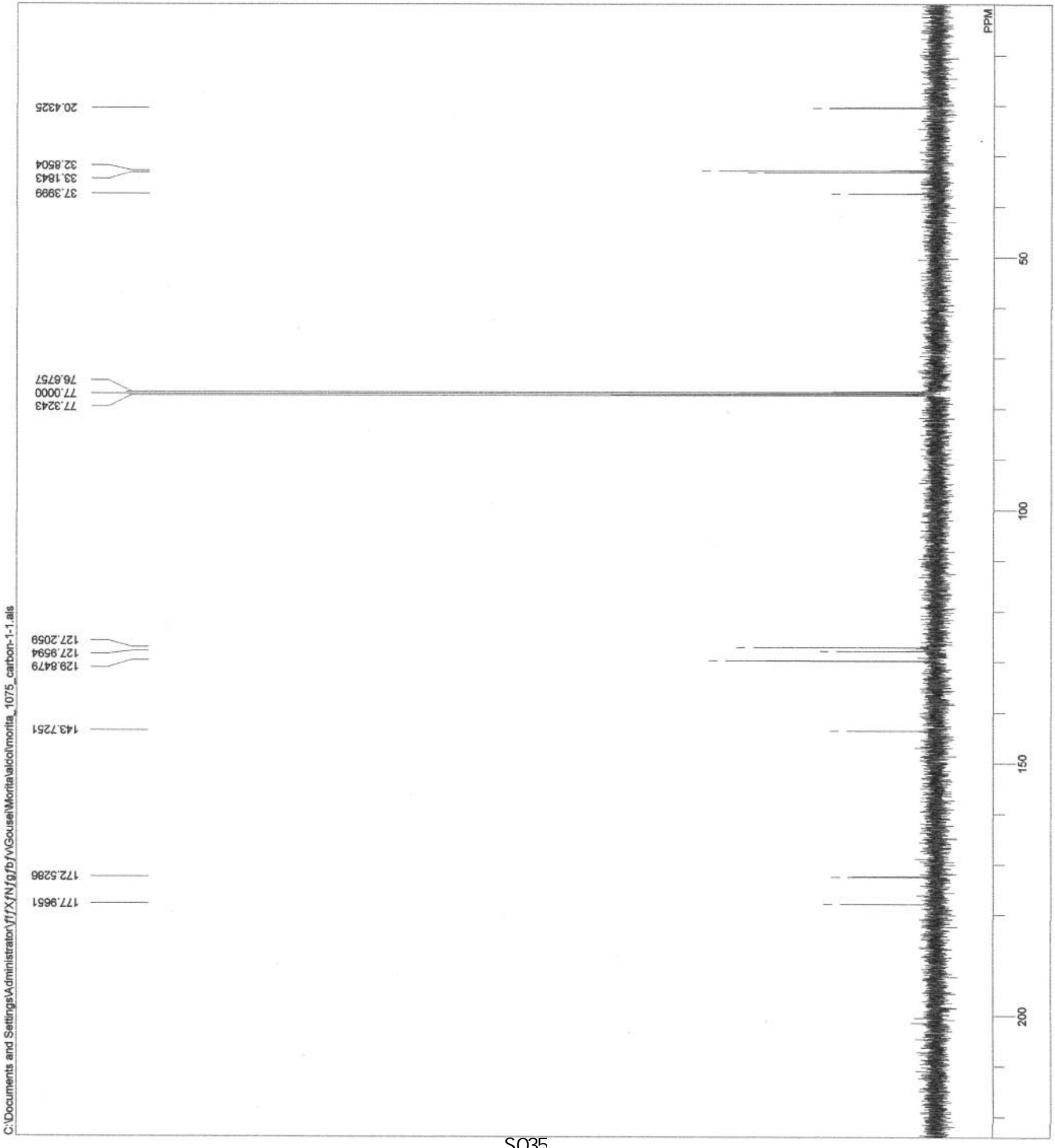




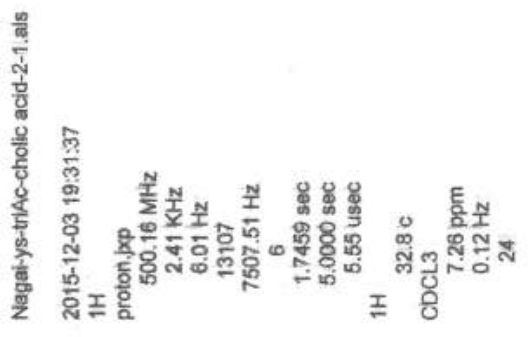

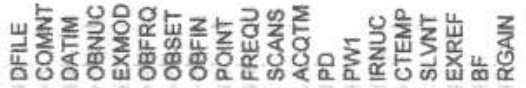
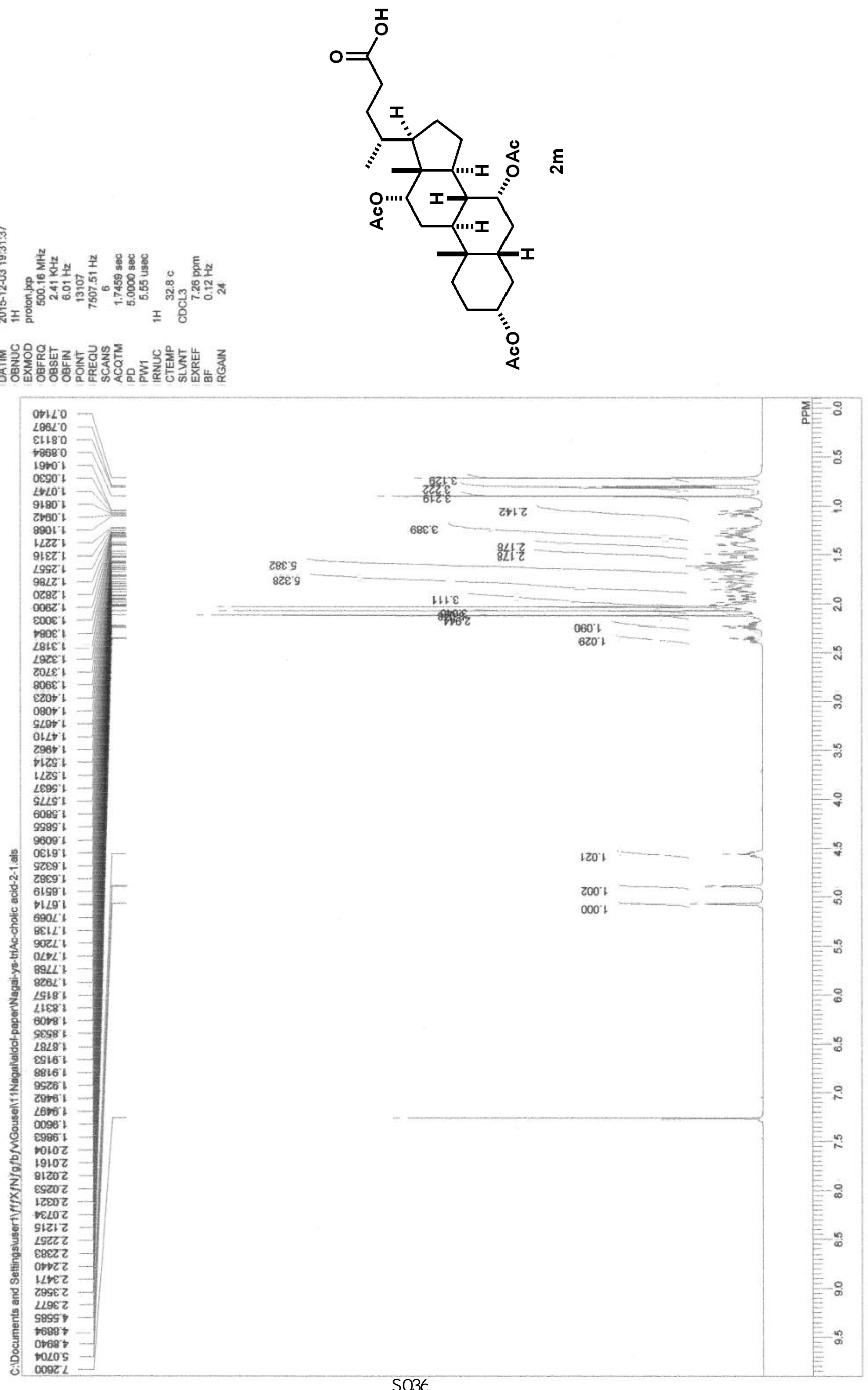


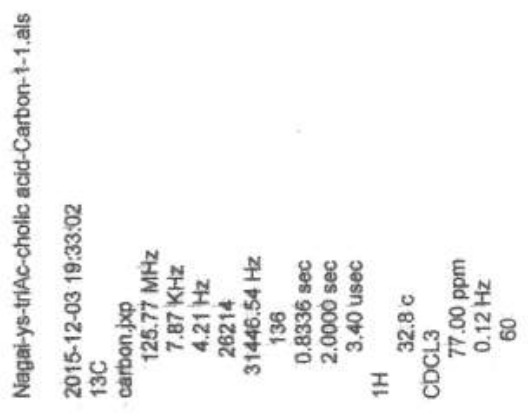

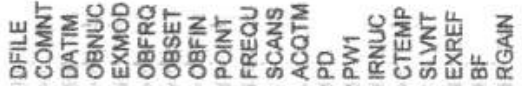
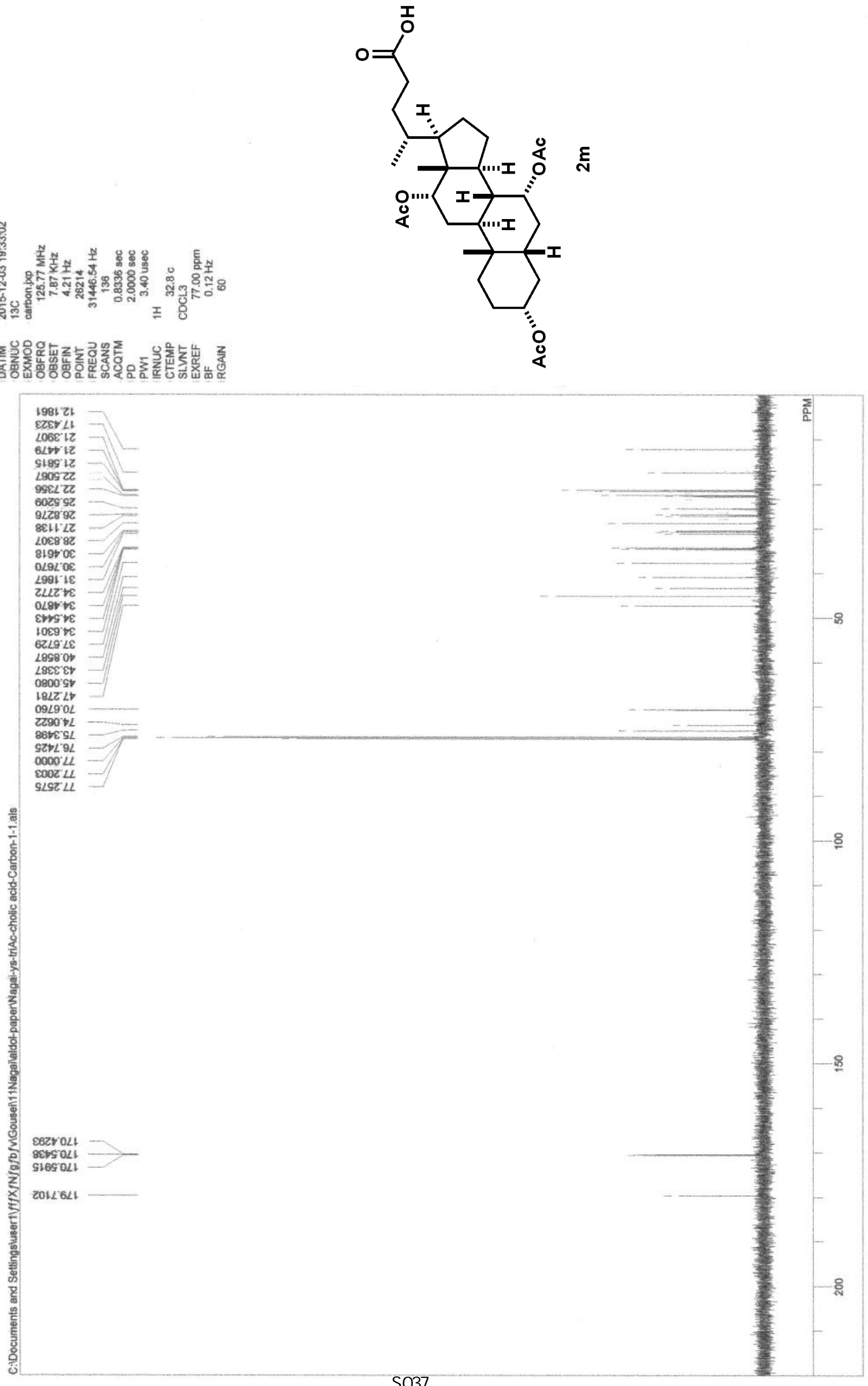

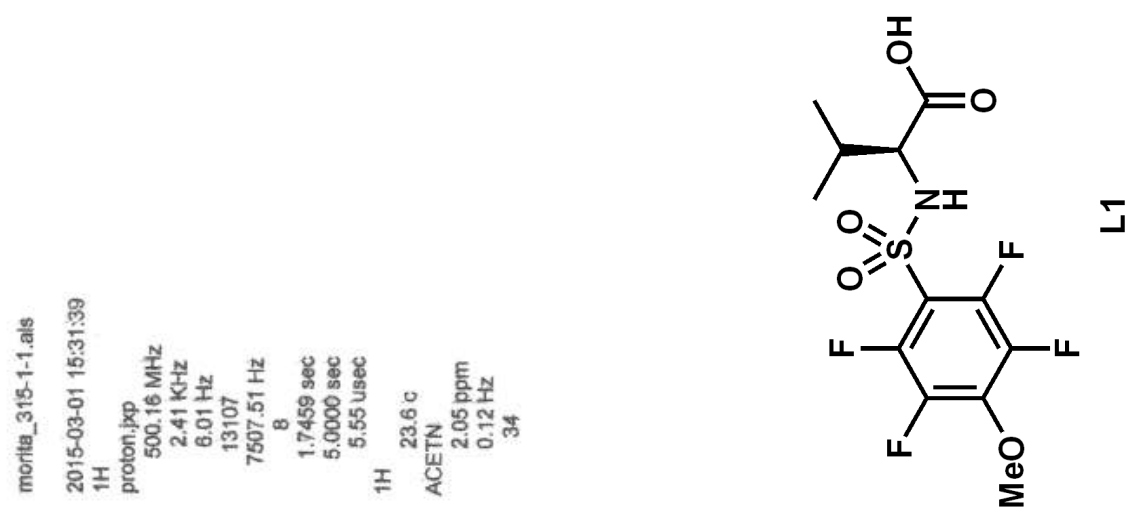

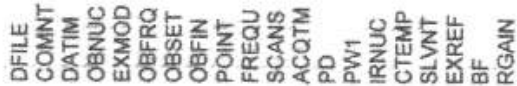

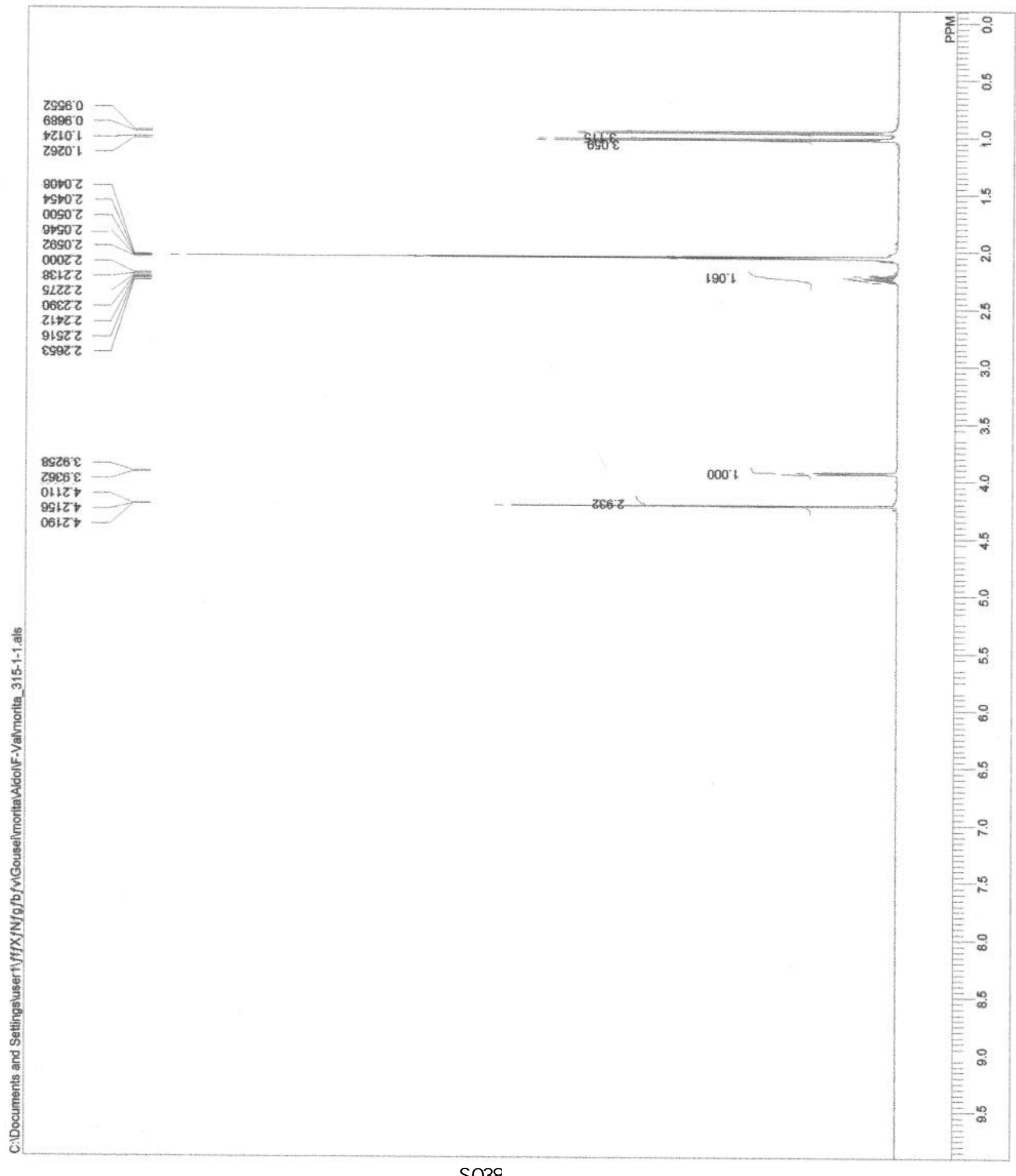



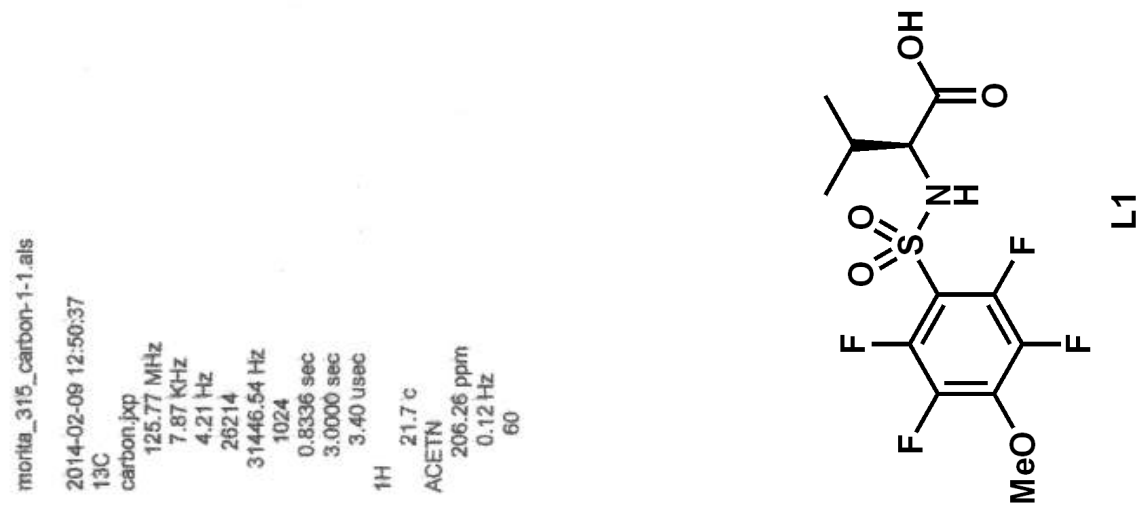

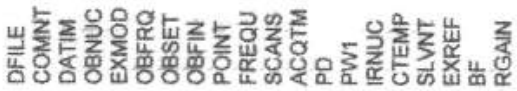

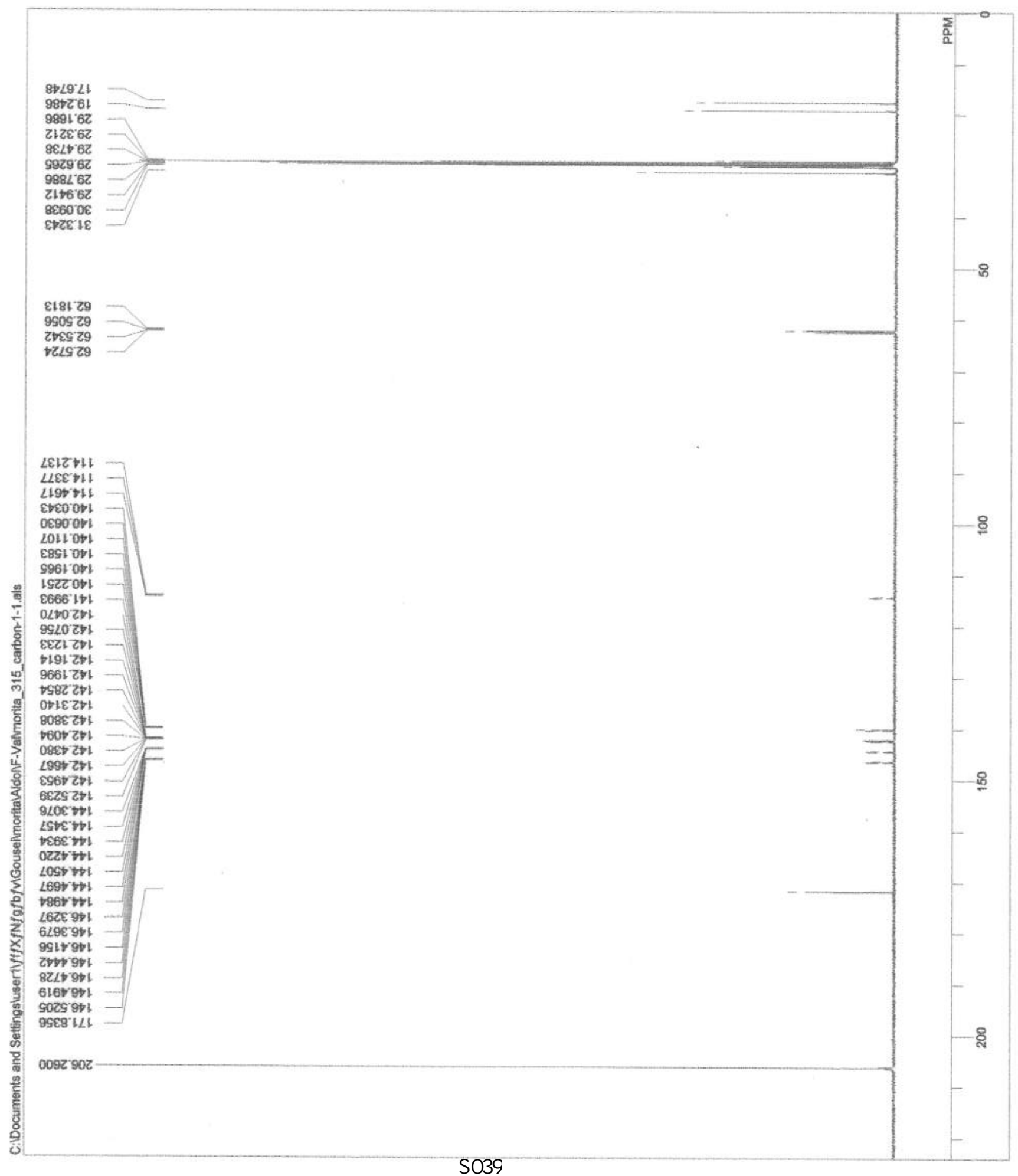




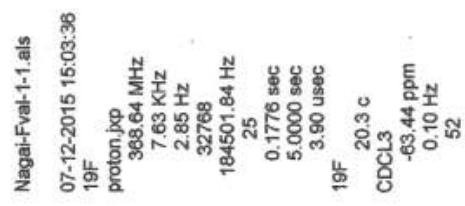

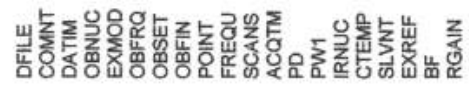

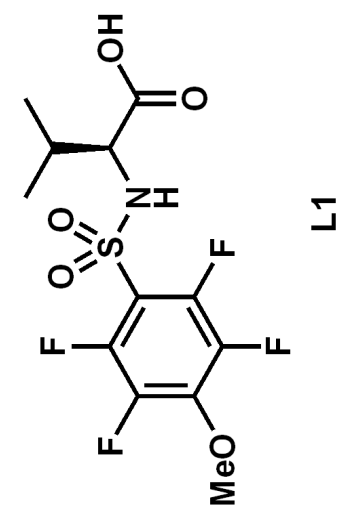

7150 2S1-

$926 !$

Z6506EL-

6ะเ $681-\square$

$000^{2}$ 

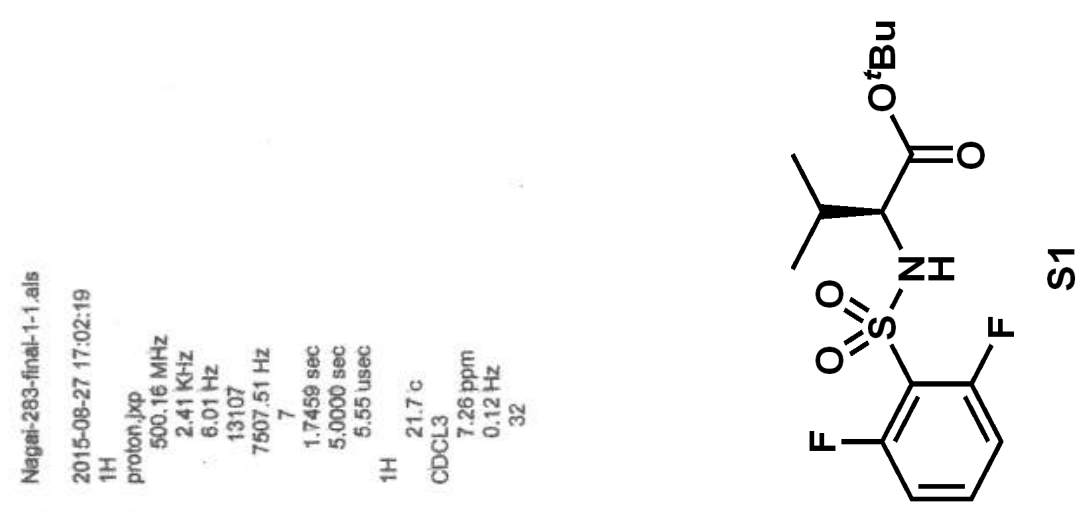

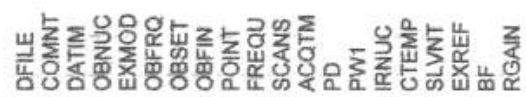

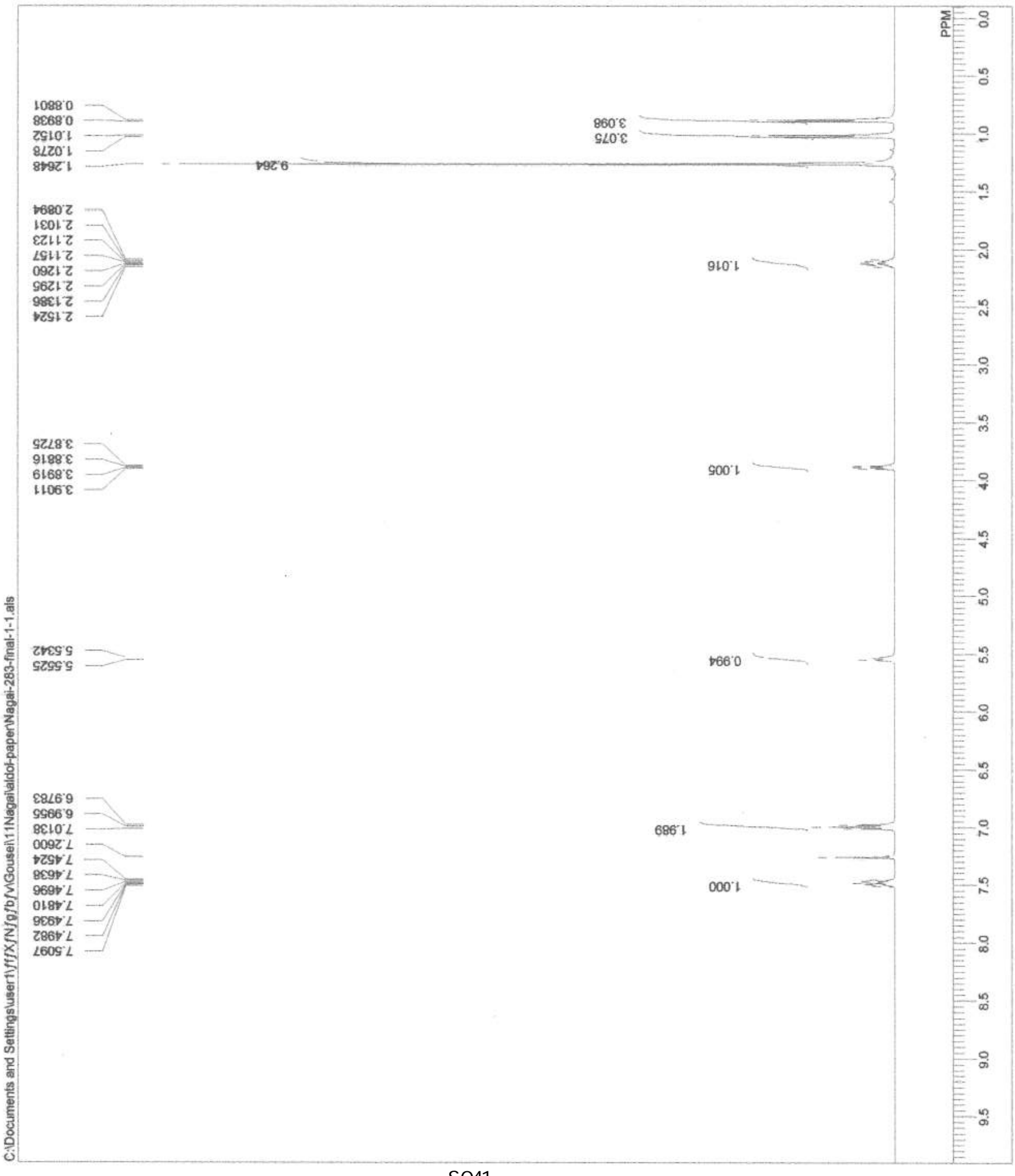



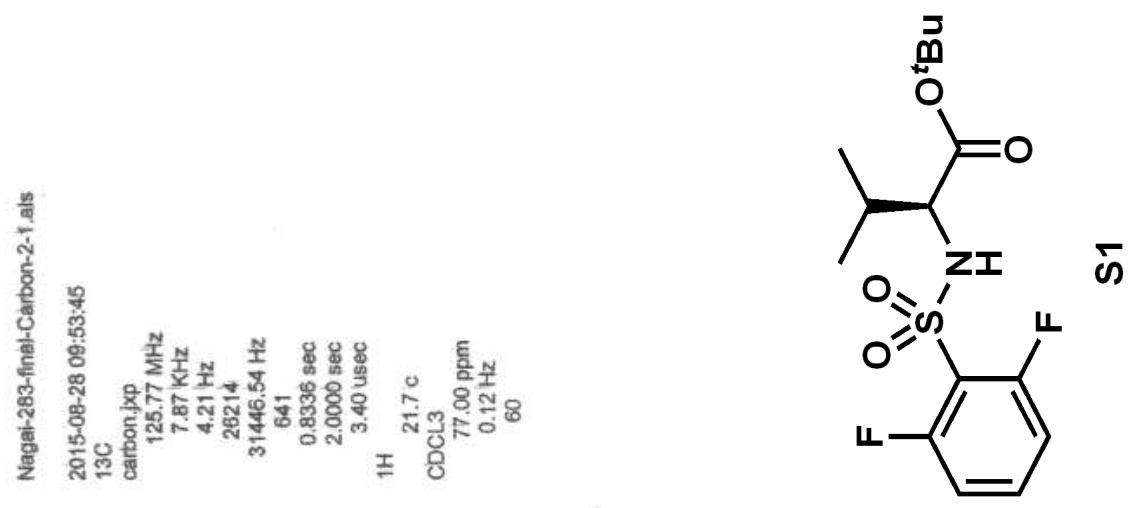

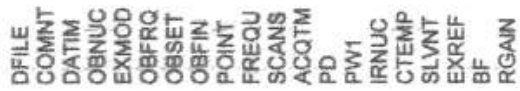

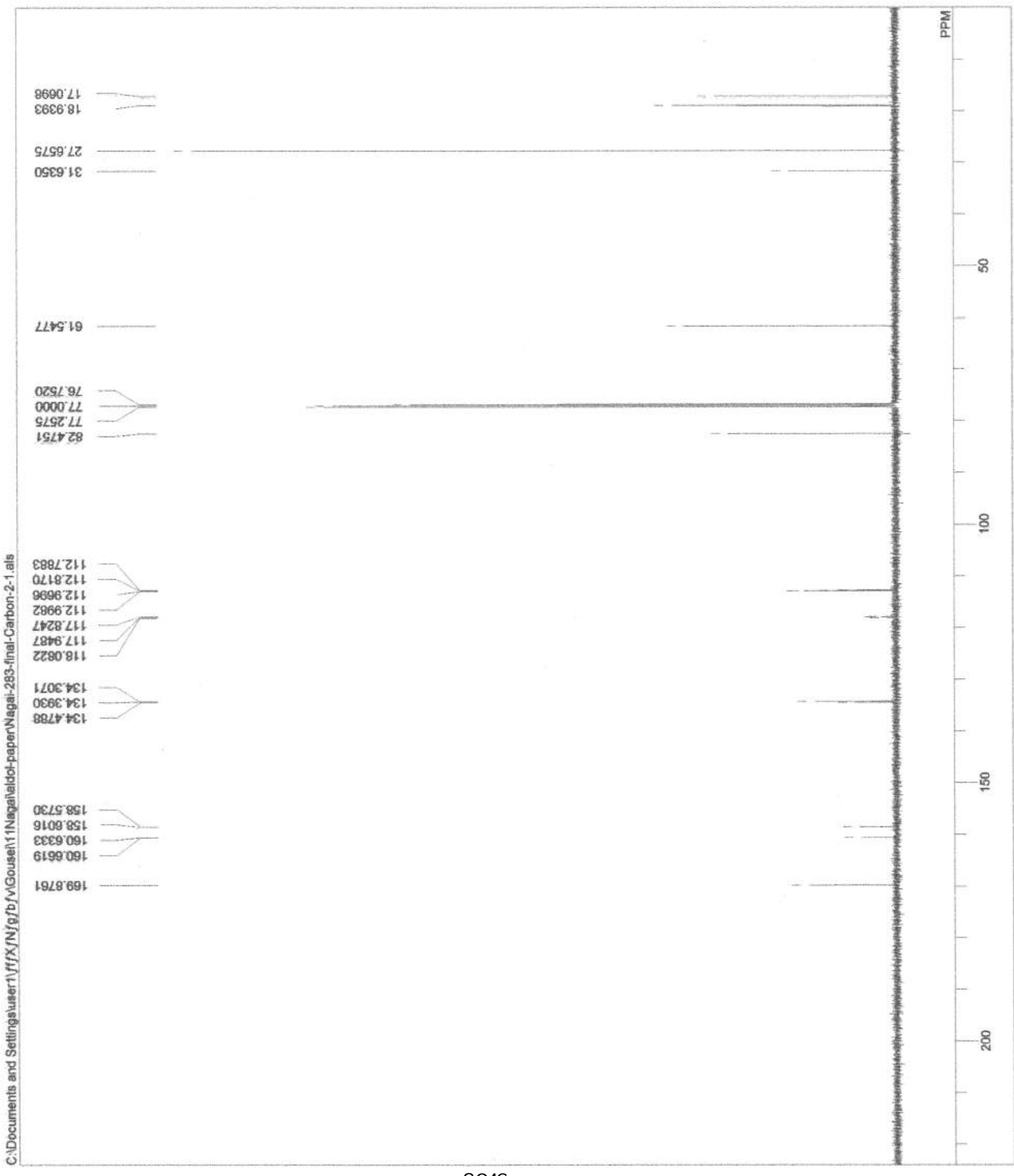




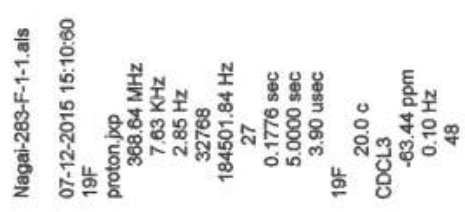

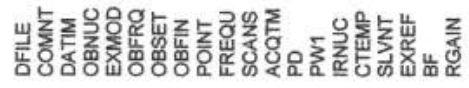
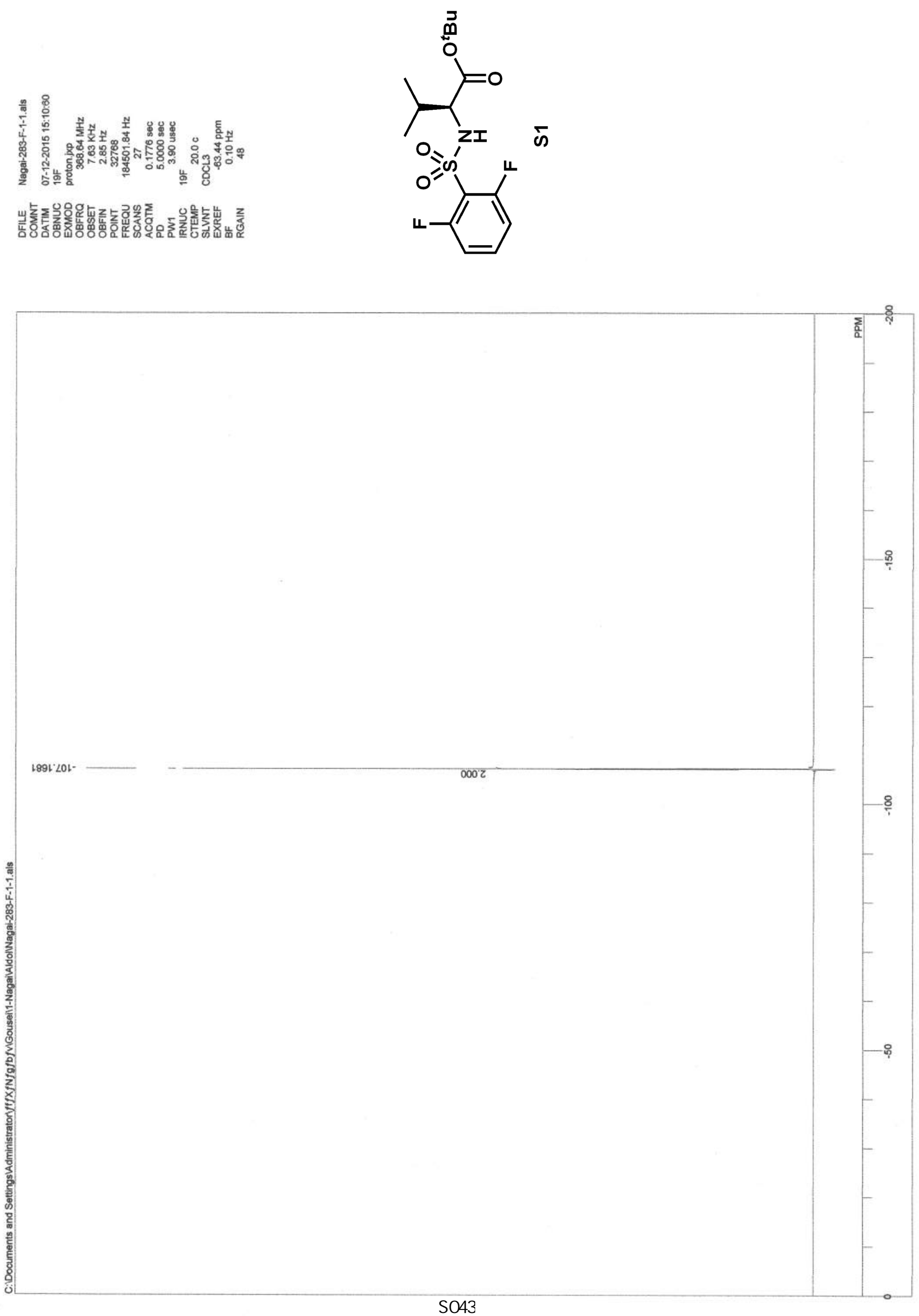

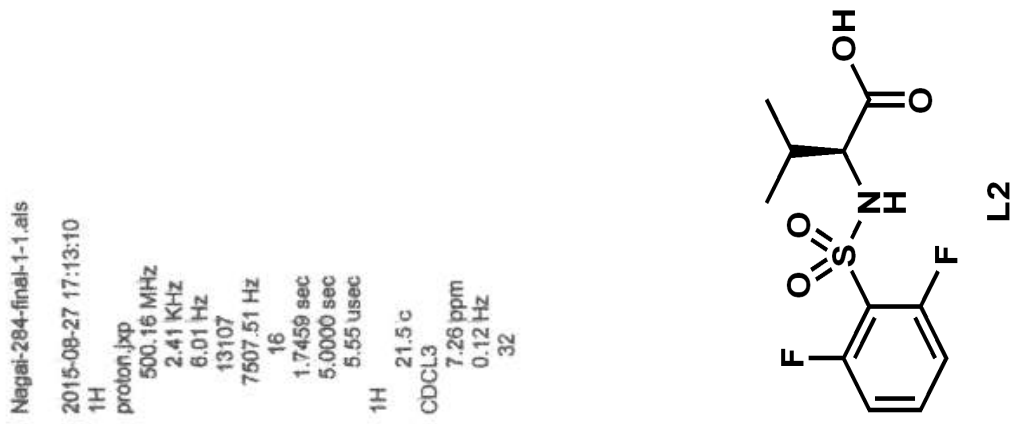

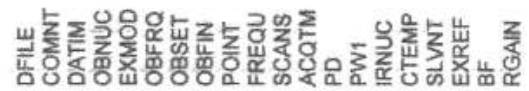

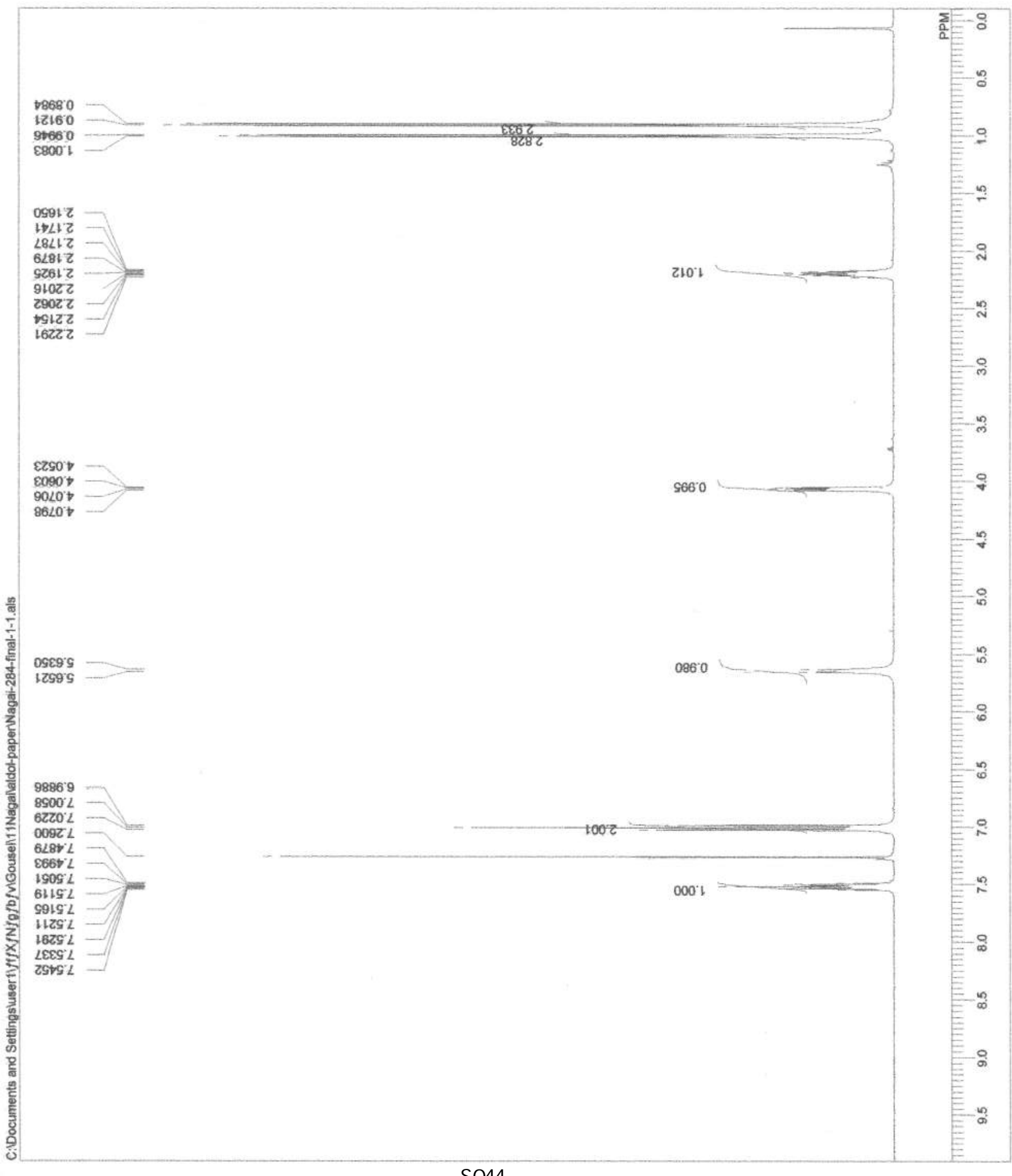



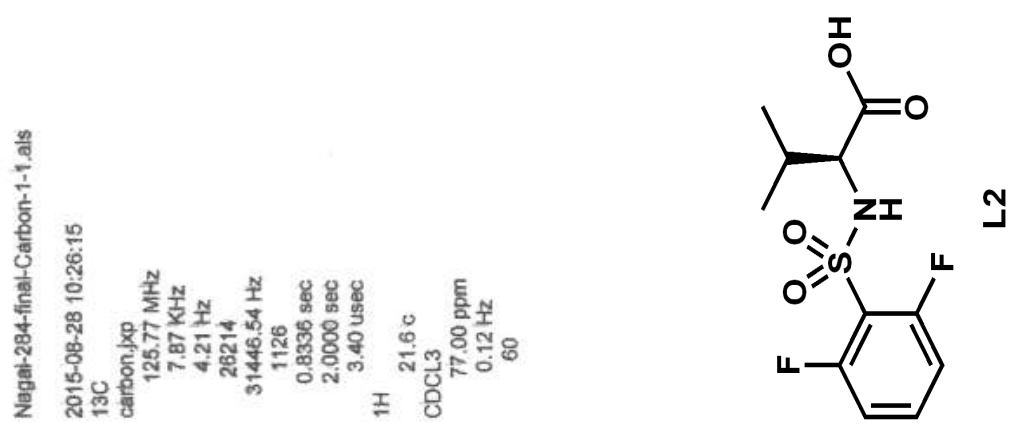

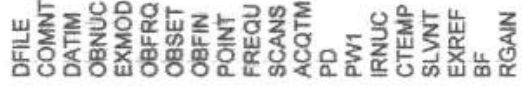

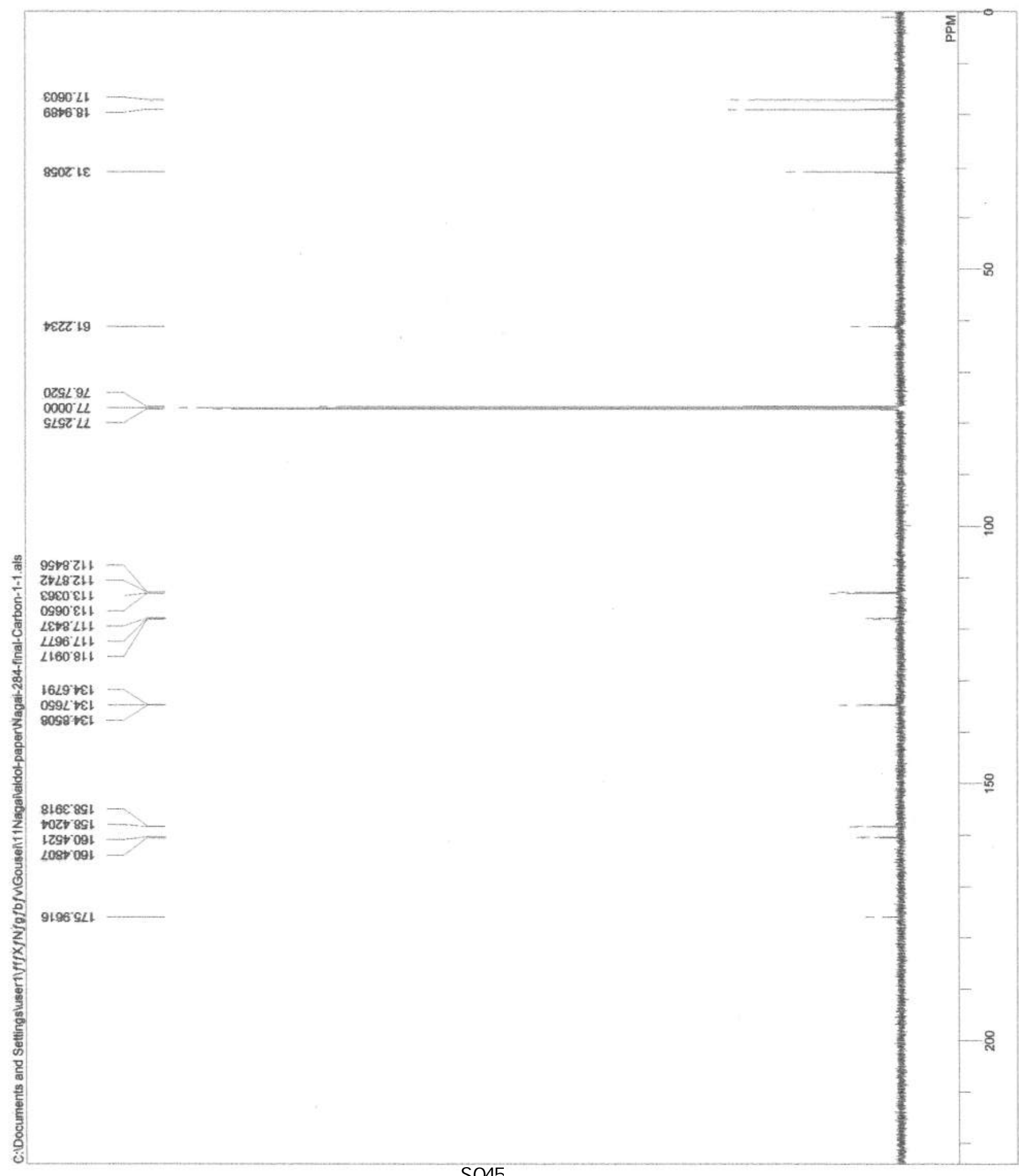




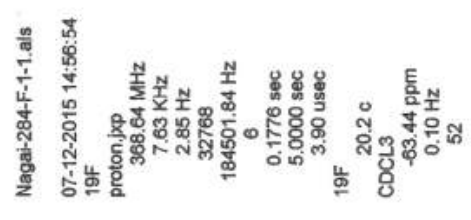

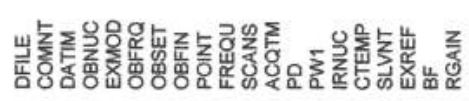
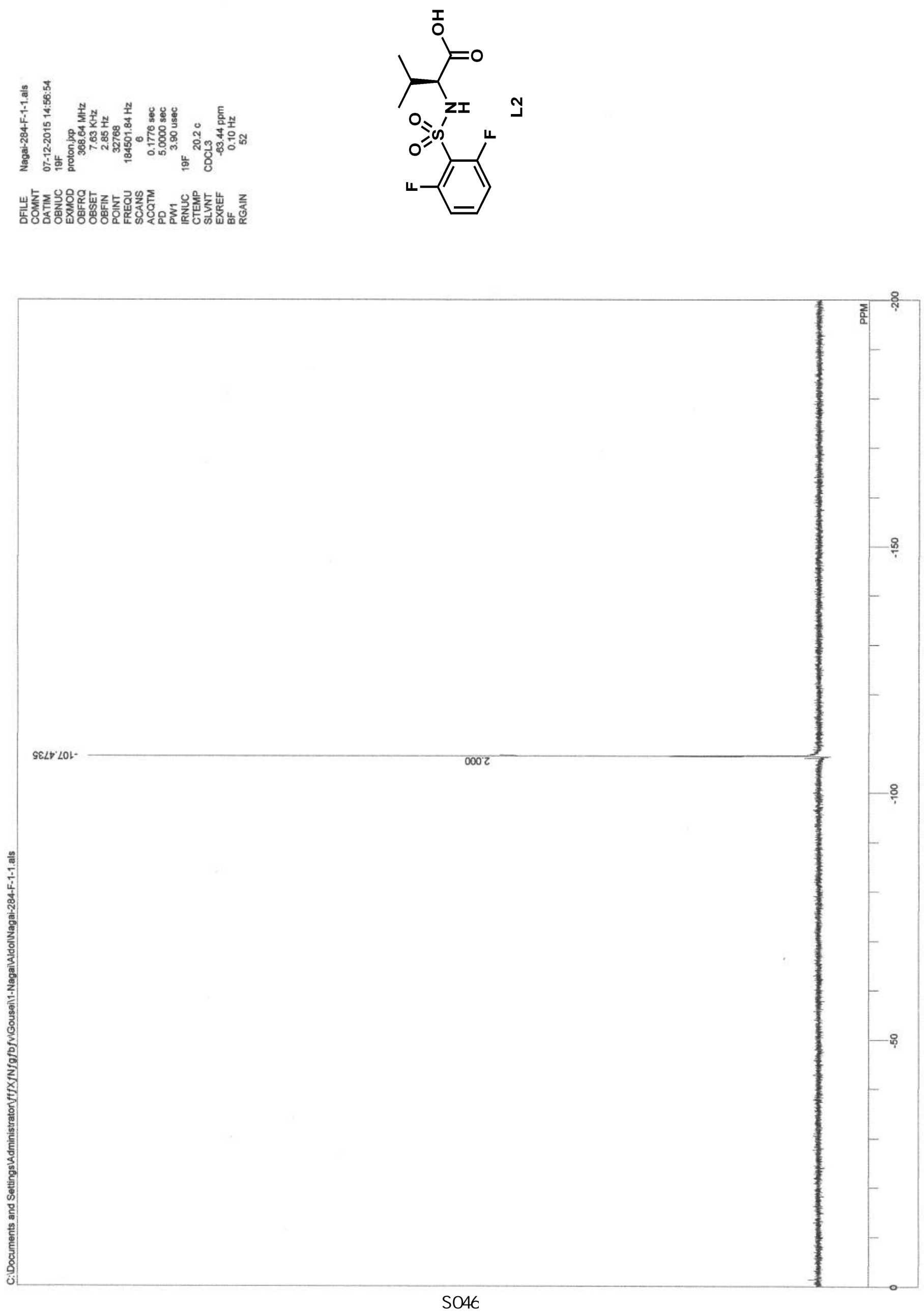

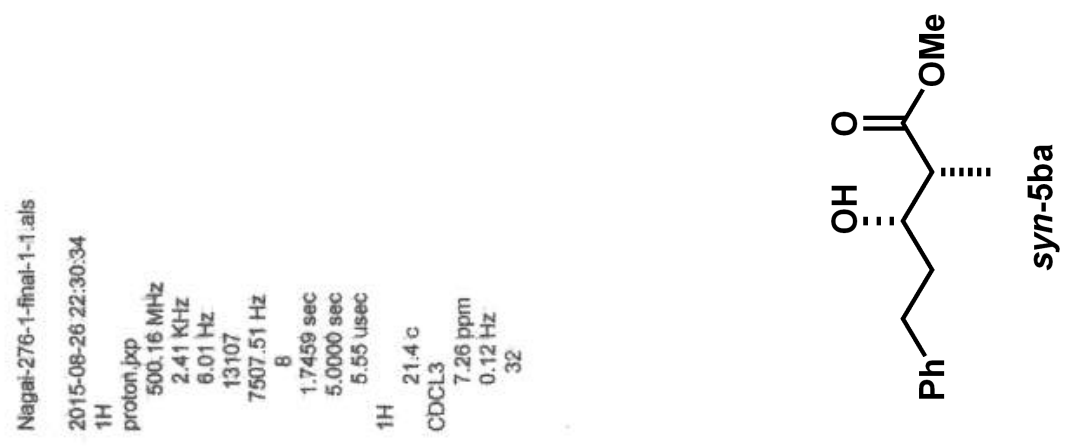

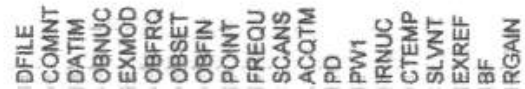

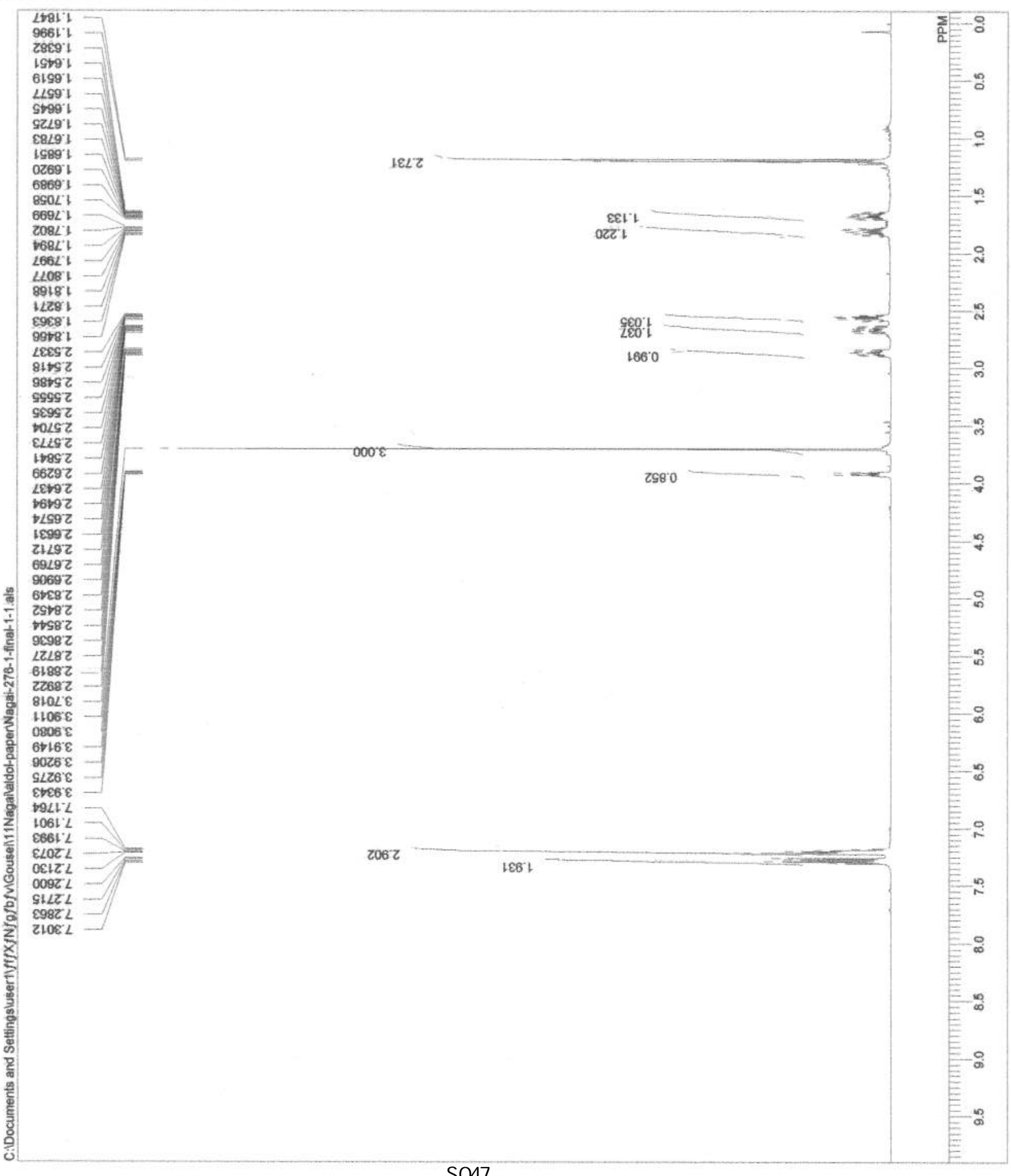




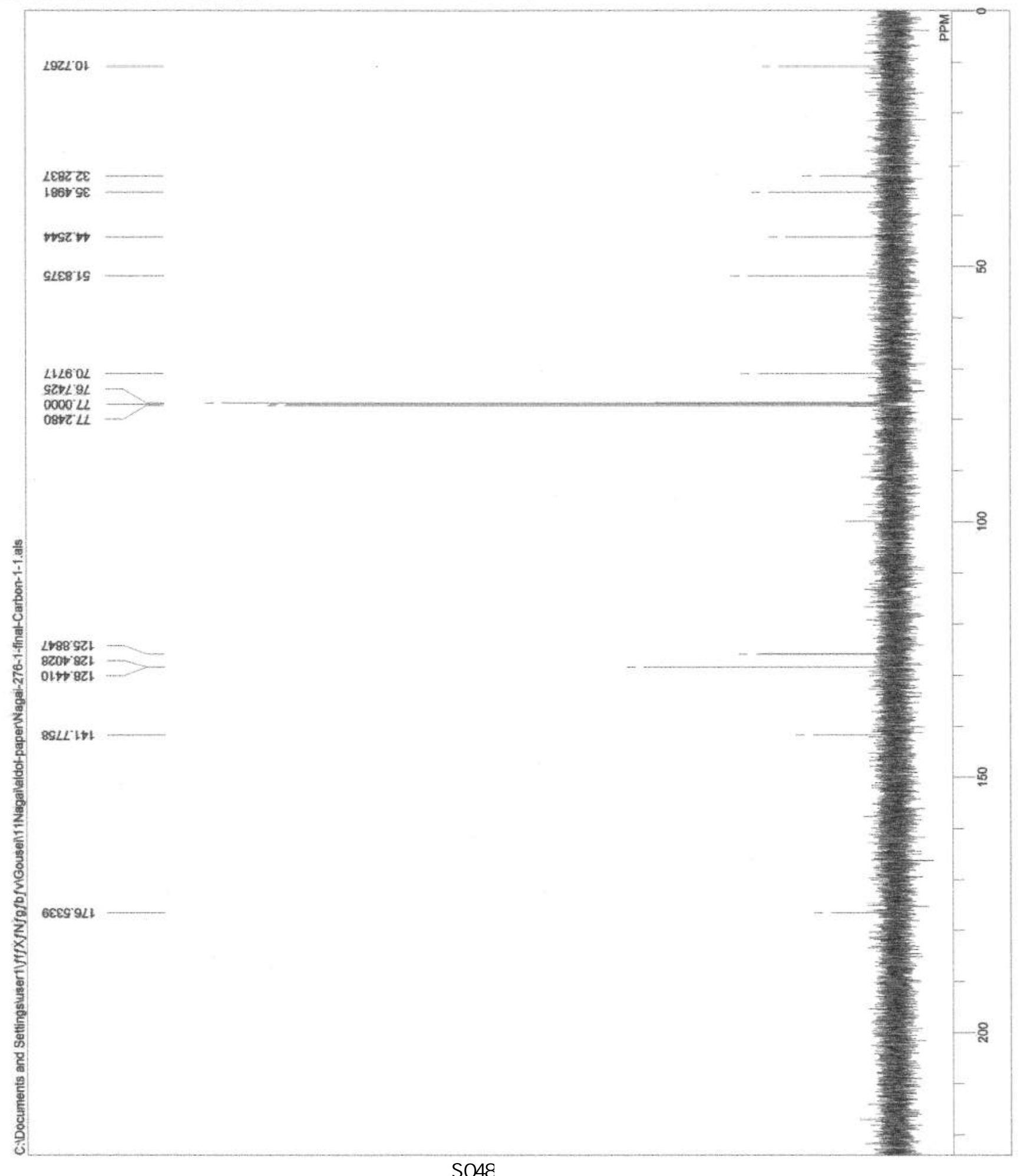



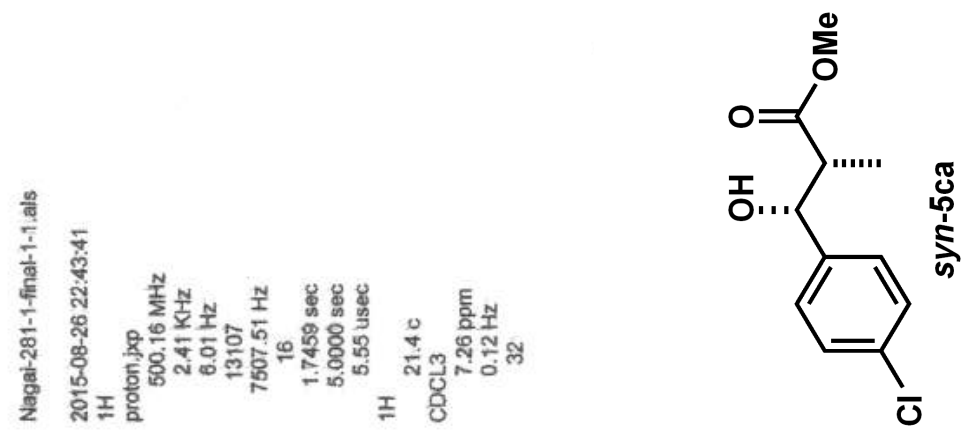

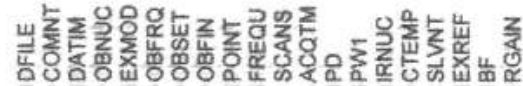

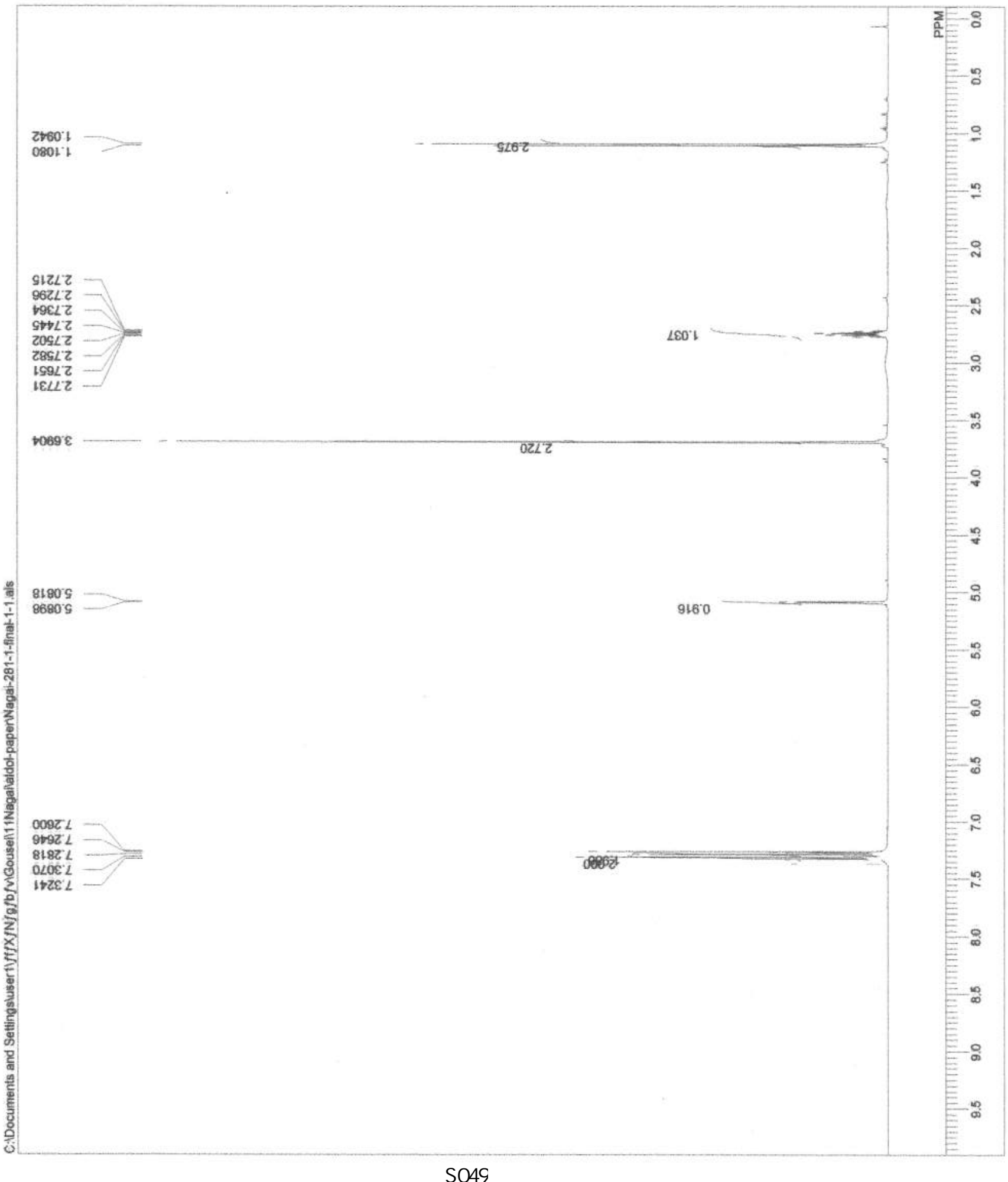



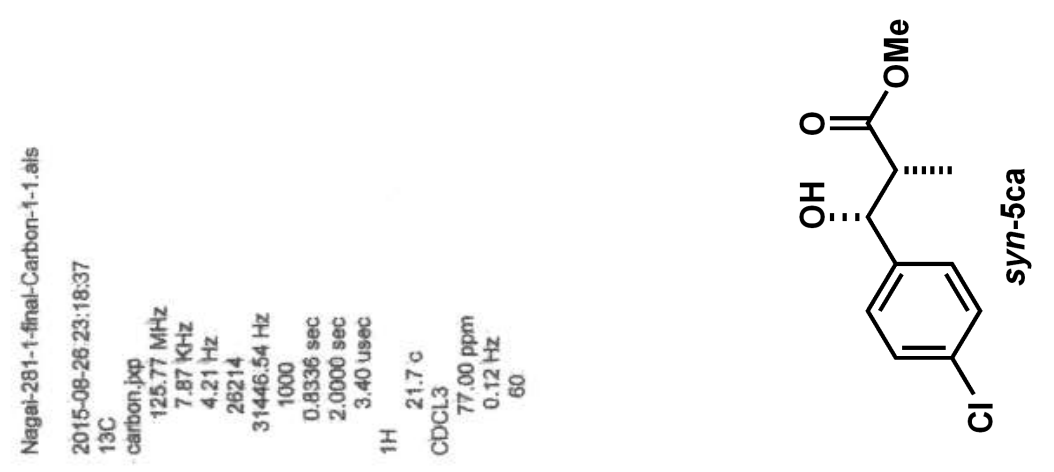

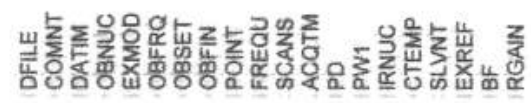

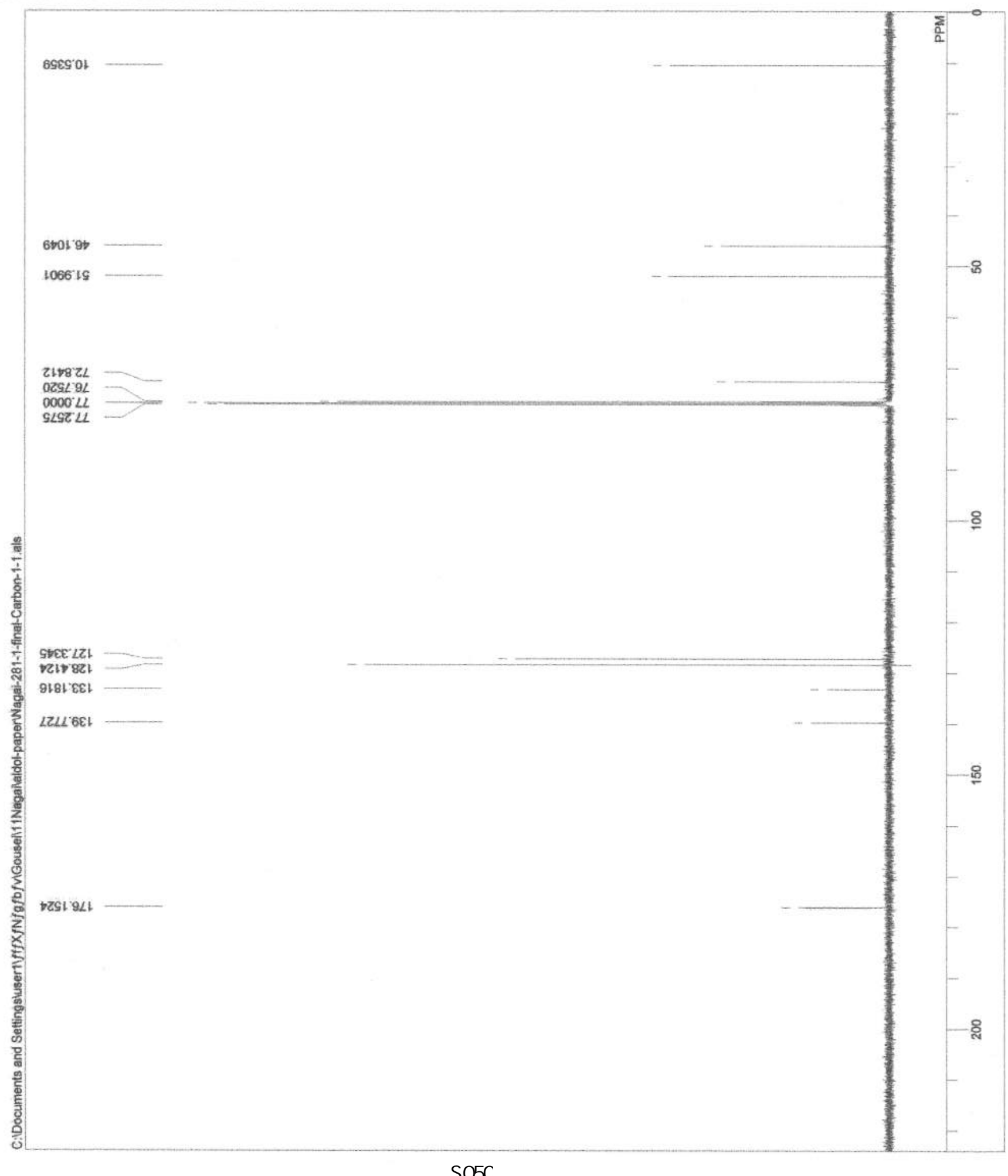



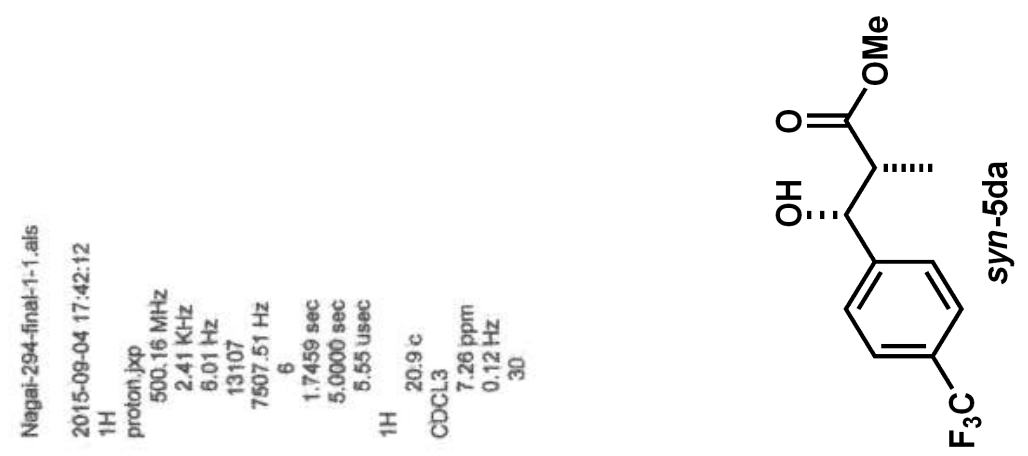

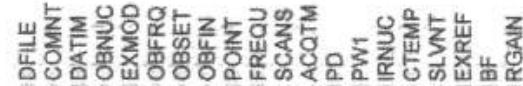

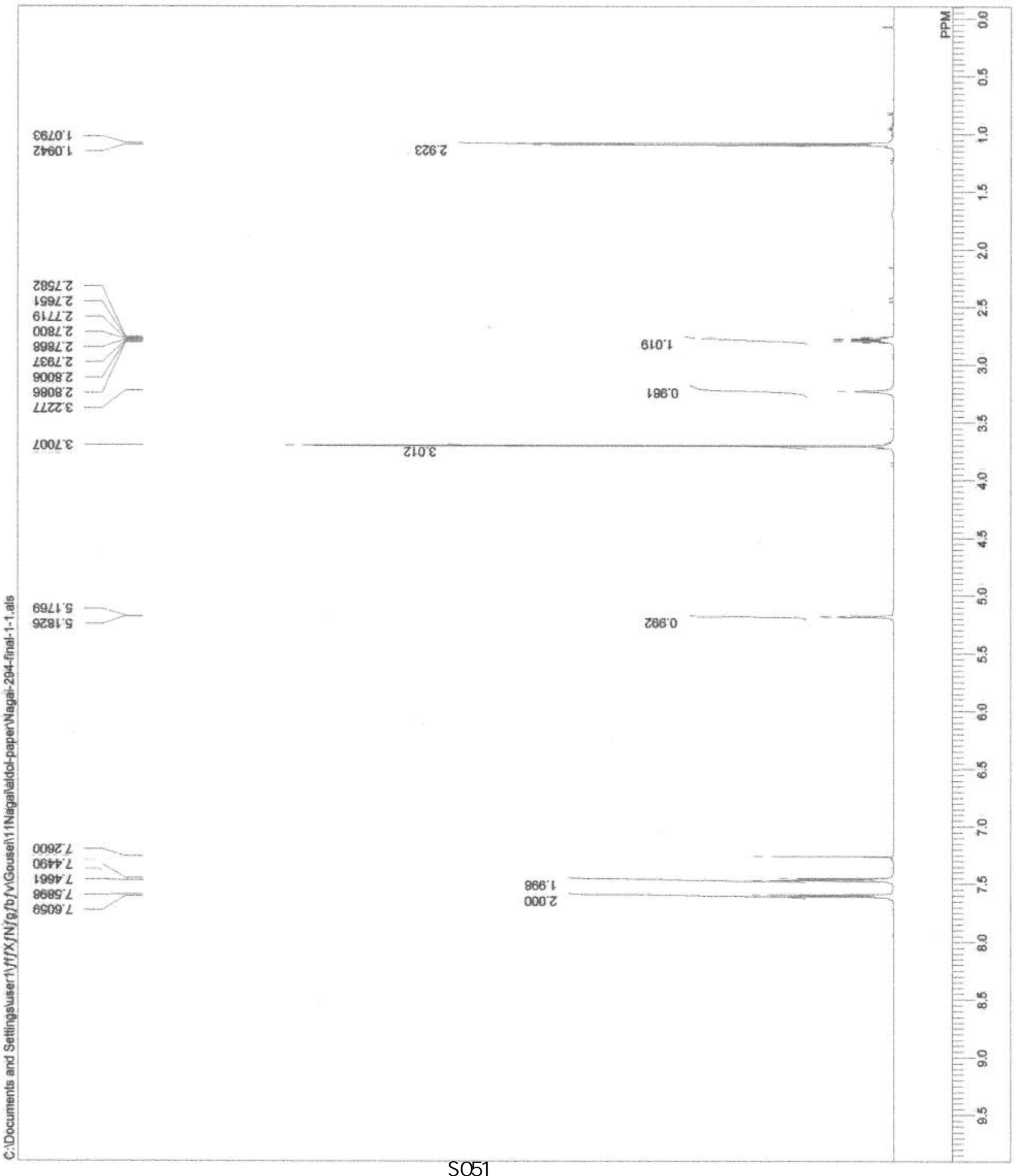



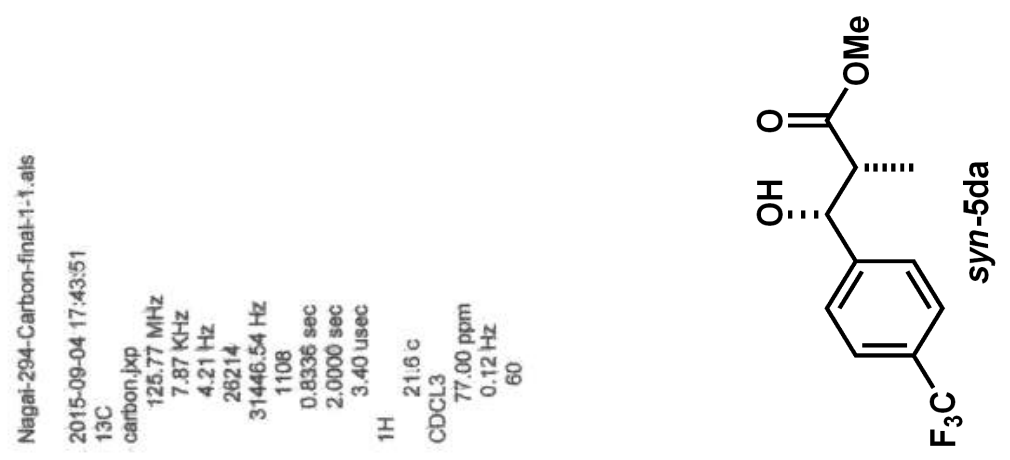

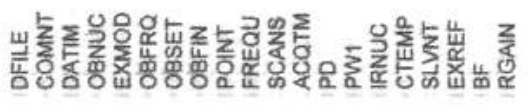

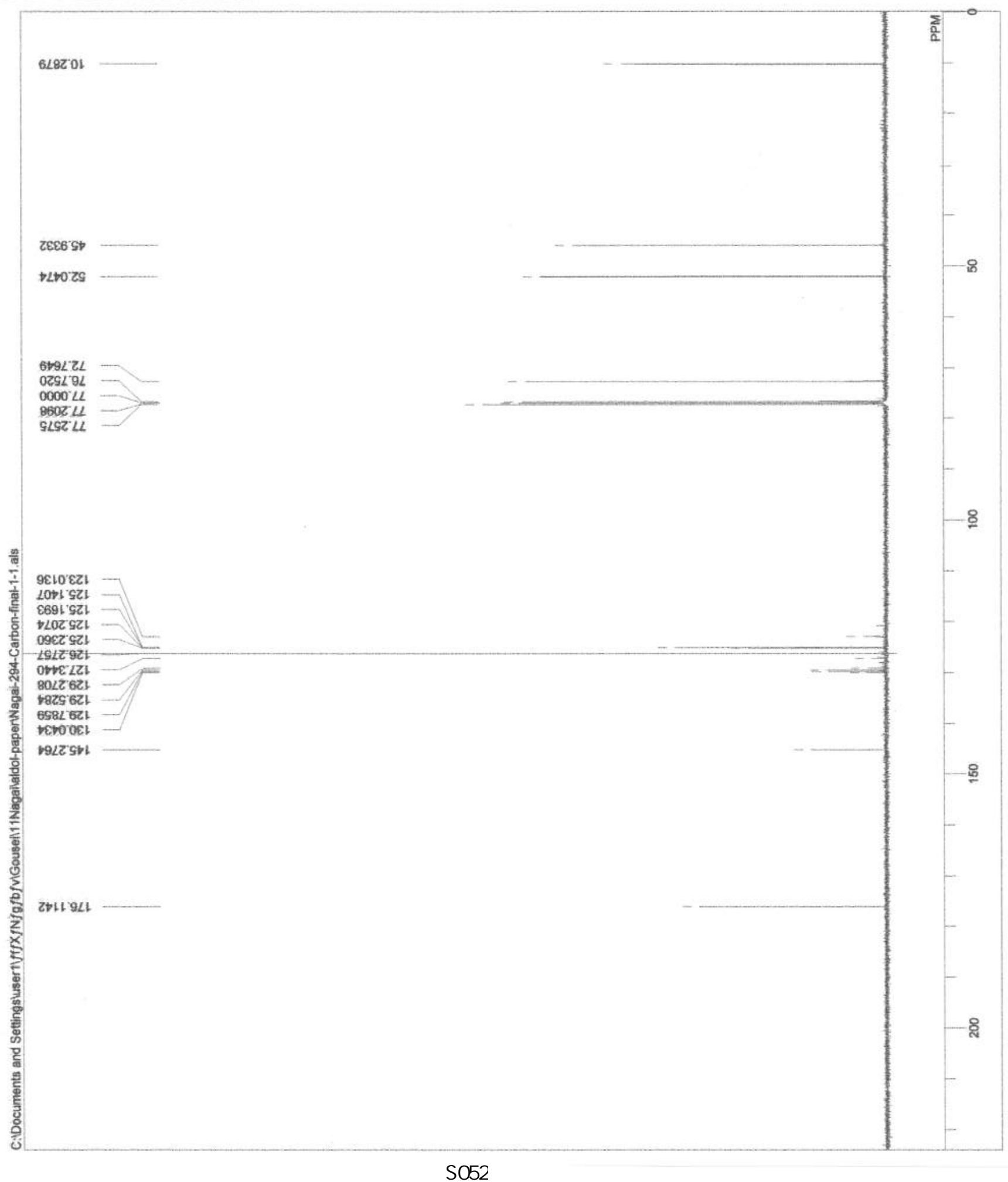




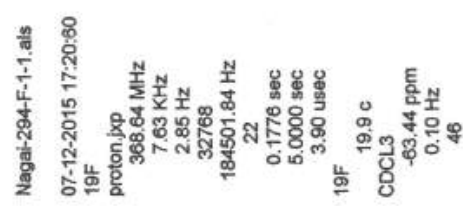

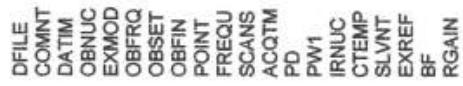

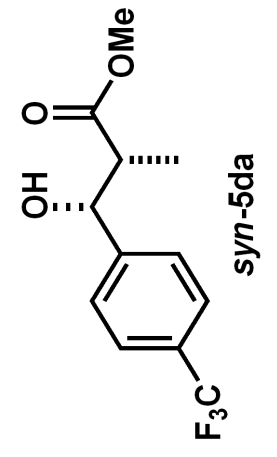

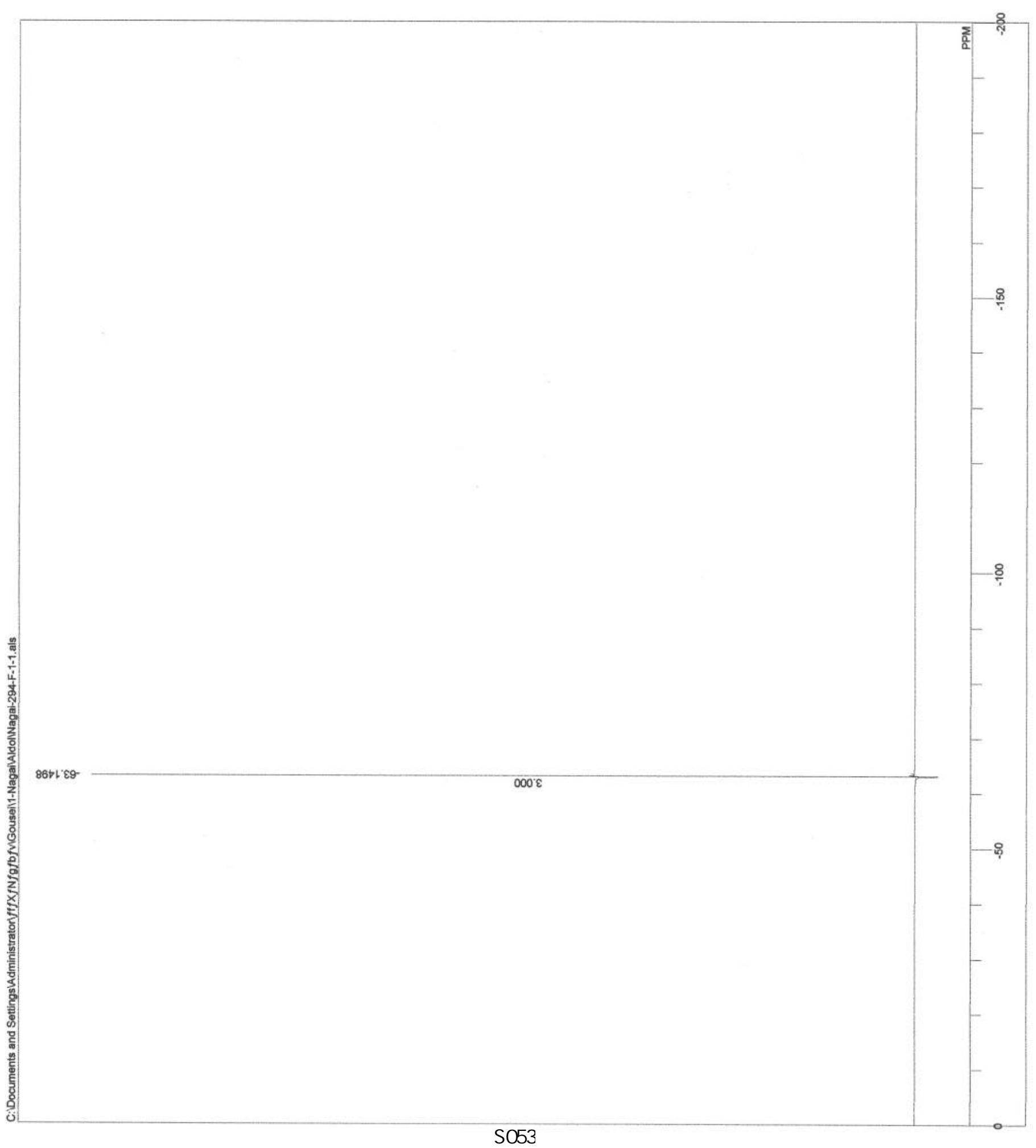



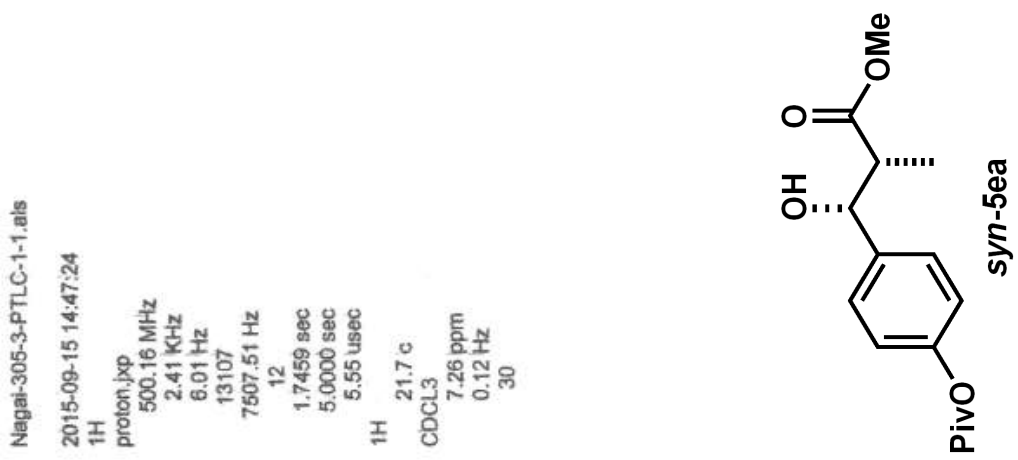

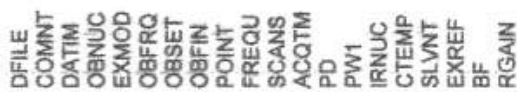

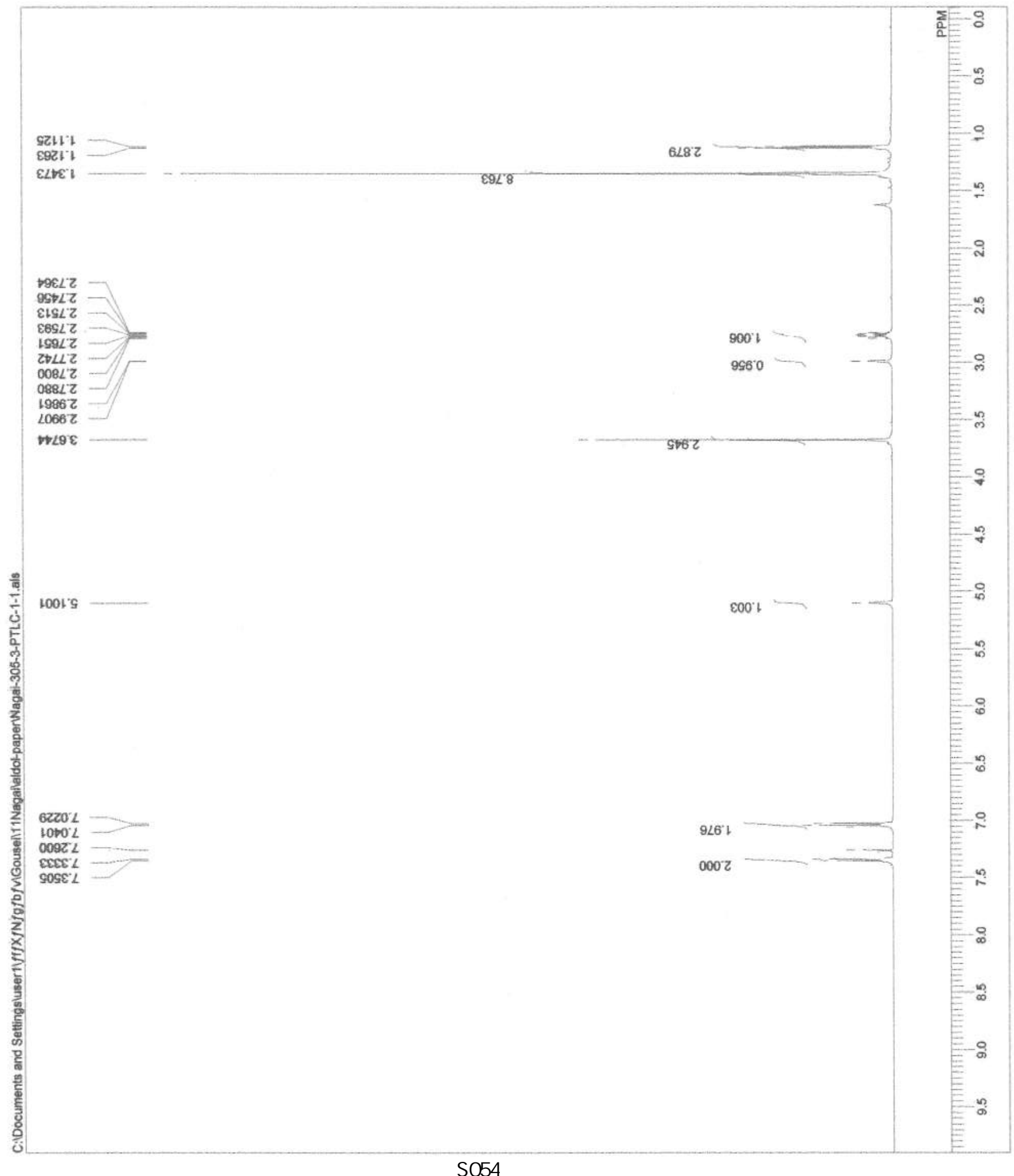



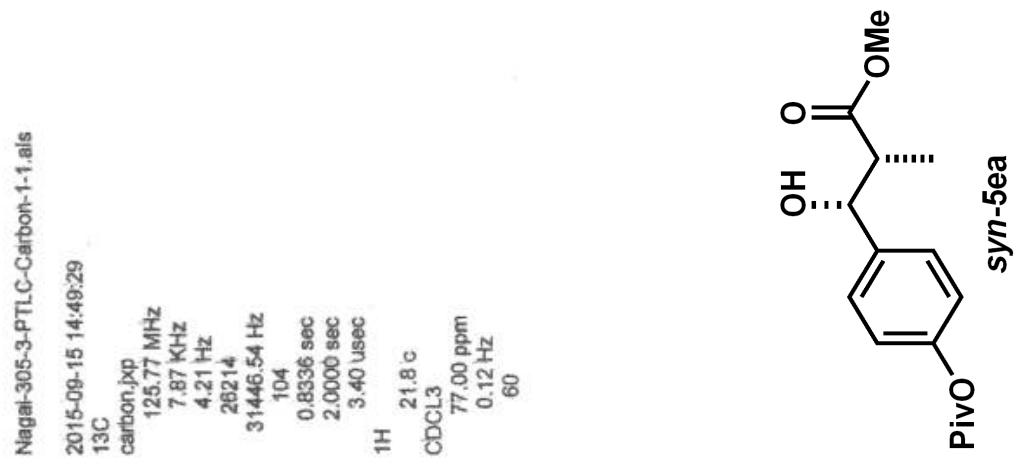

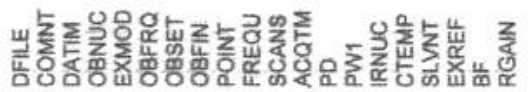

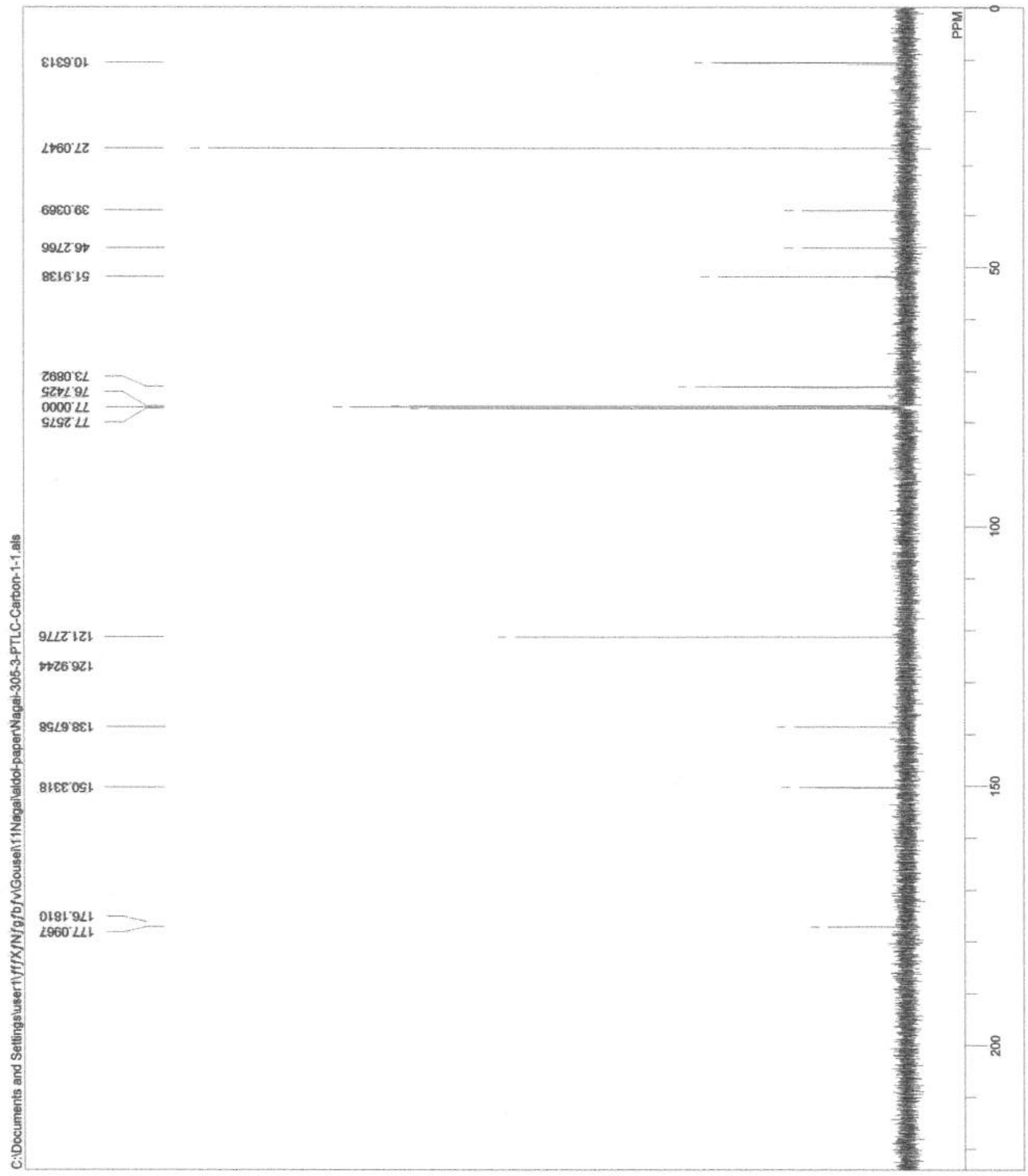



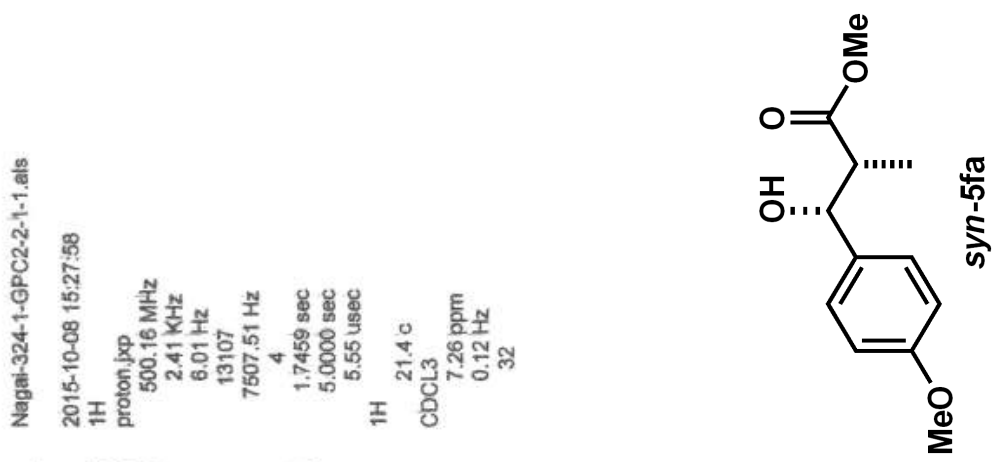

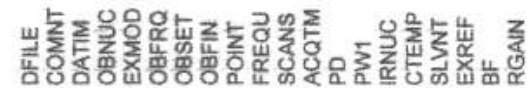

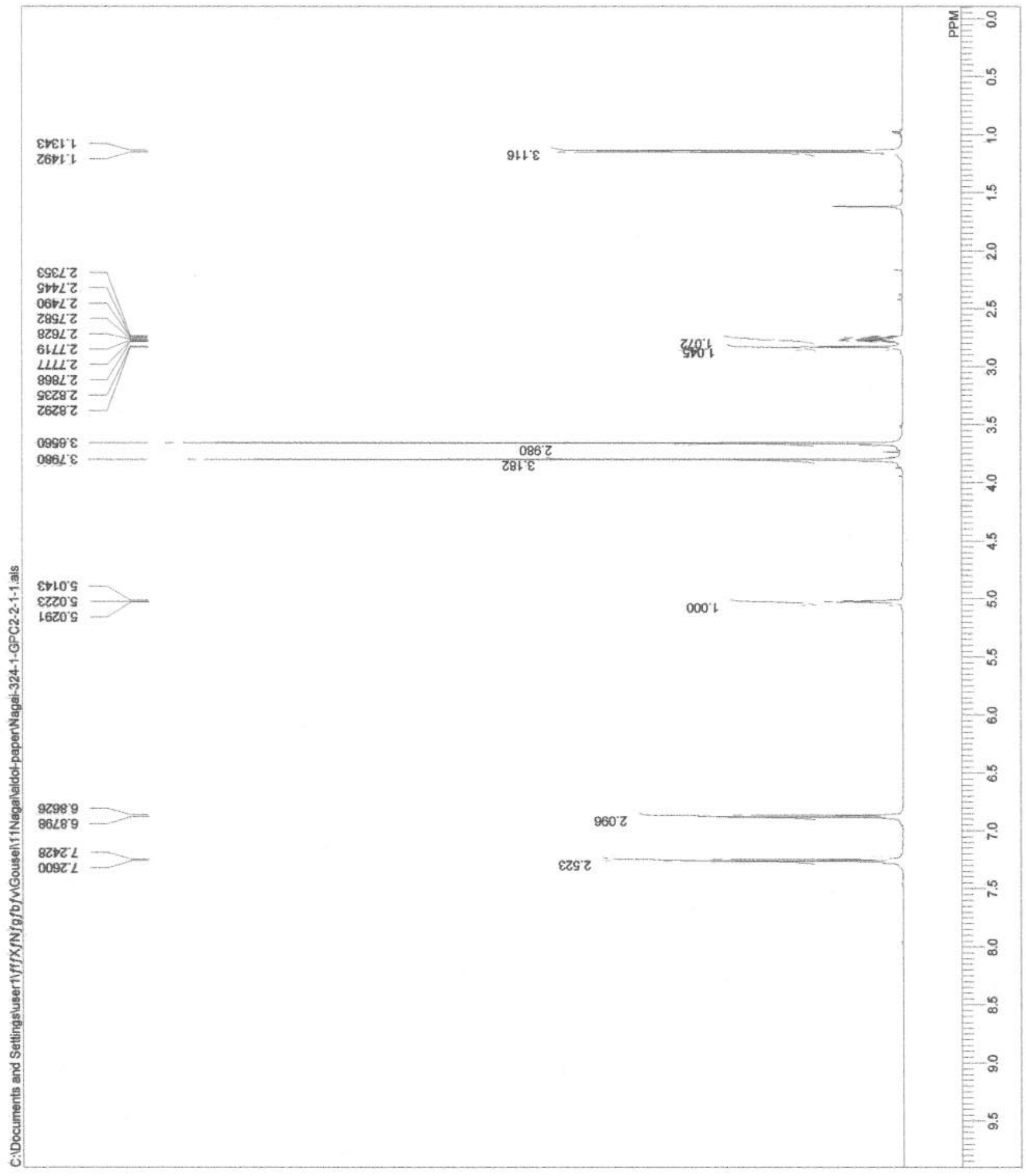



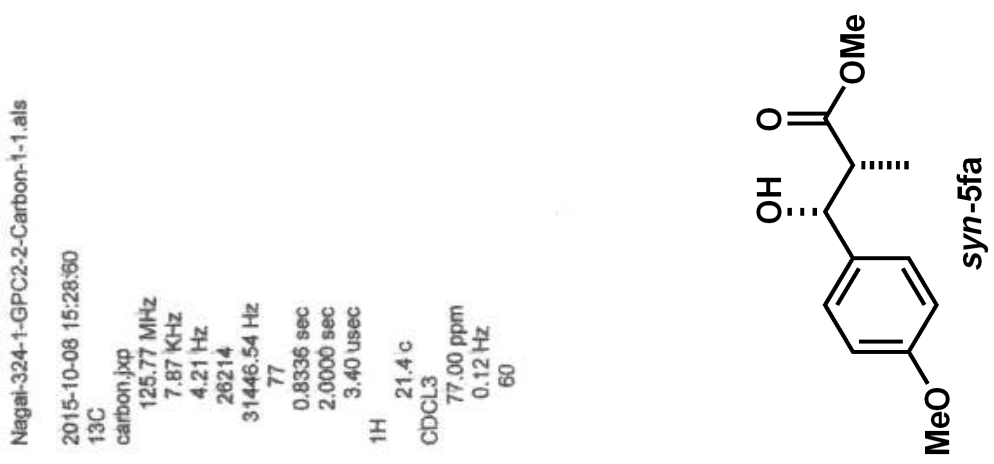

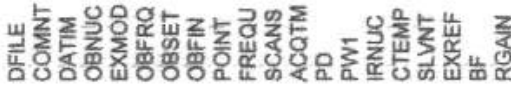

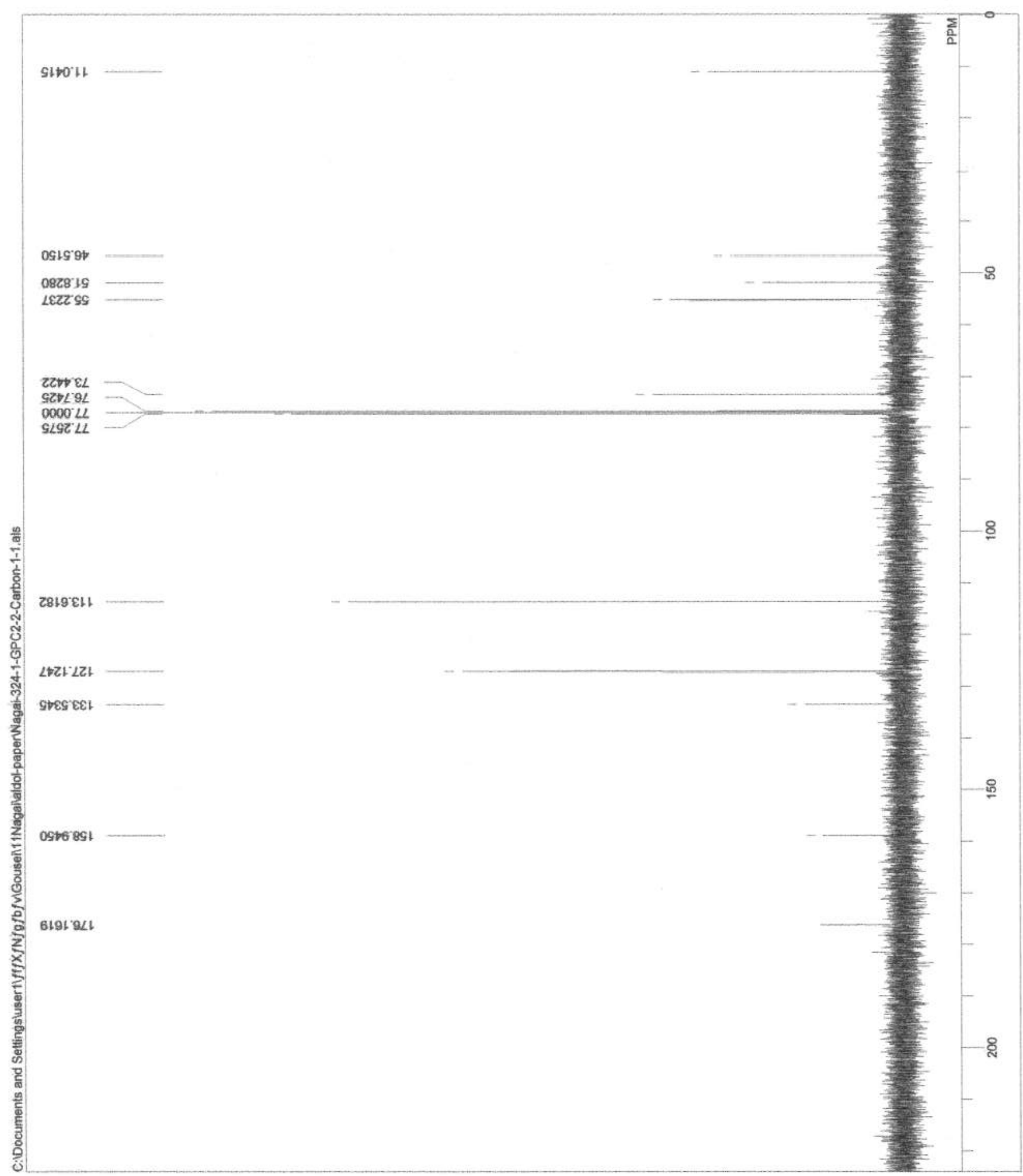



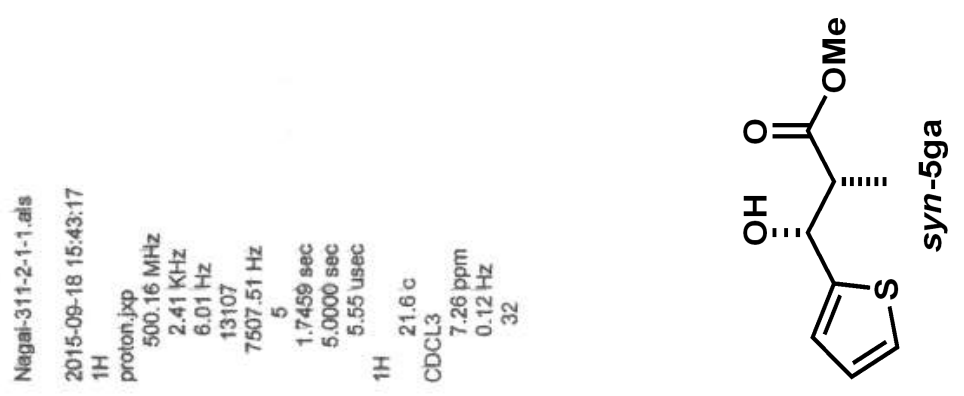

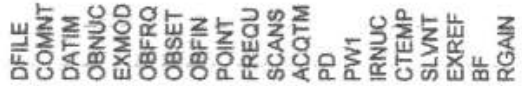

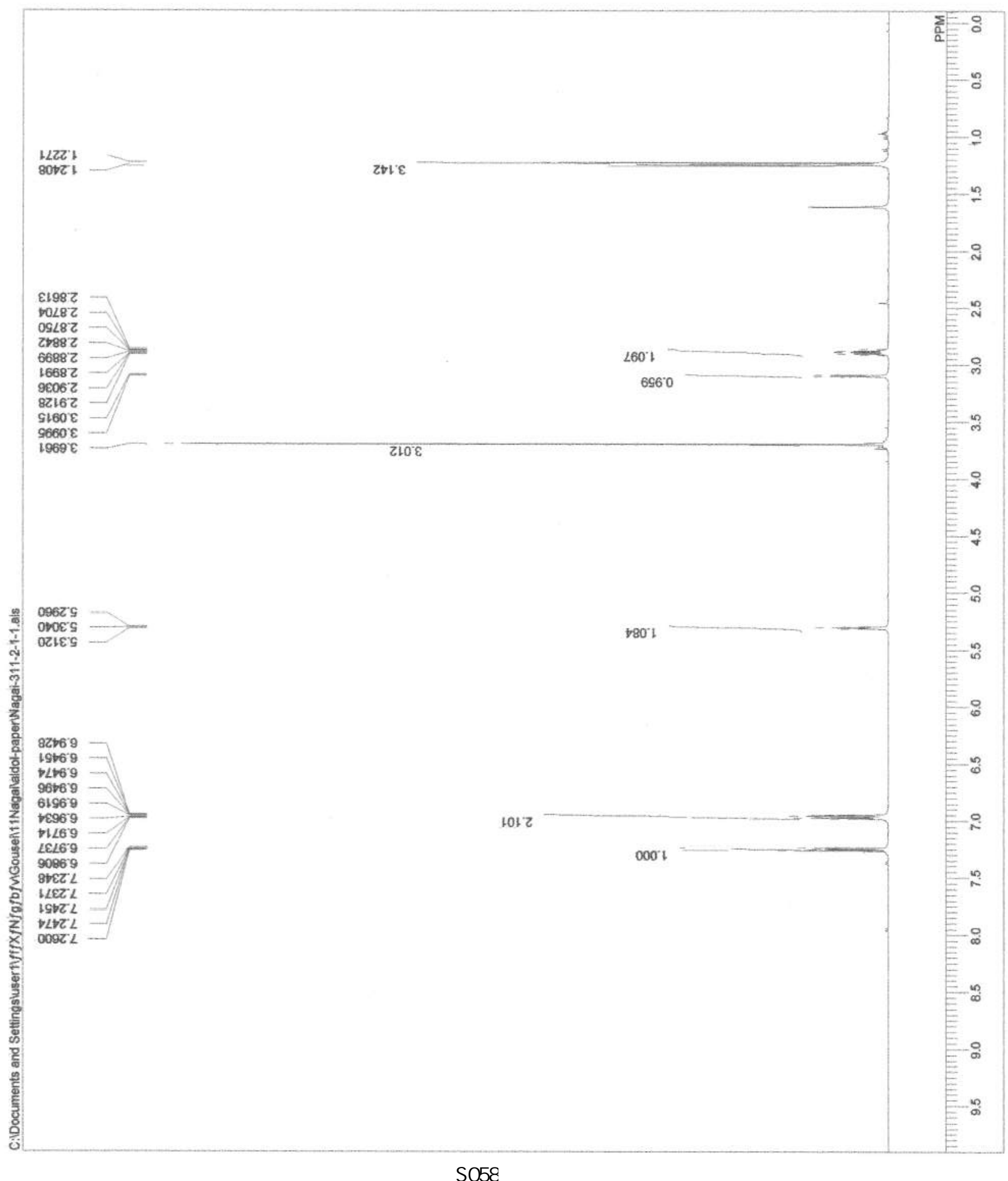



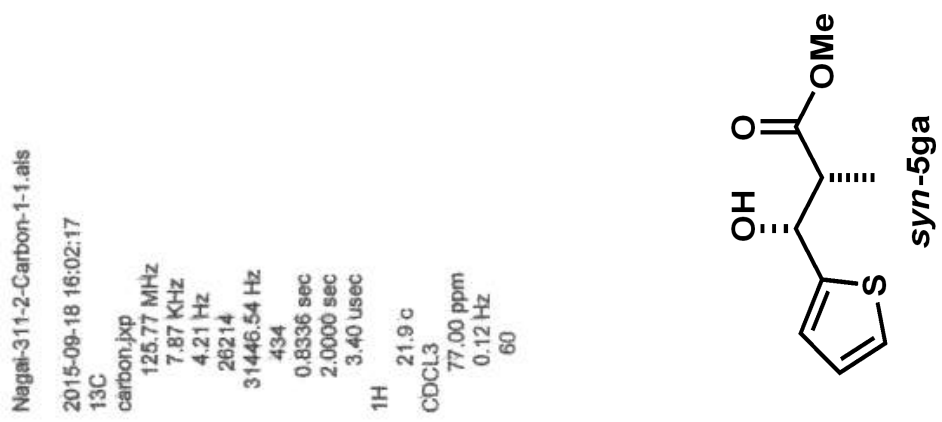

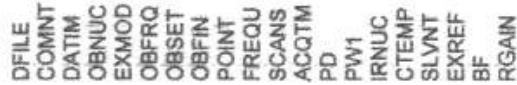

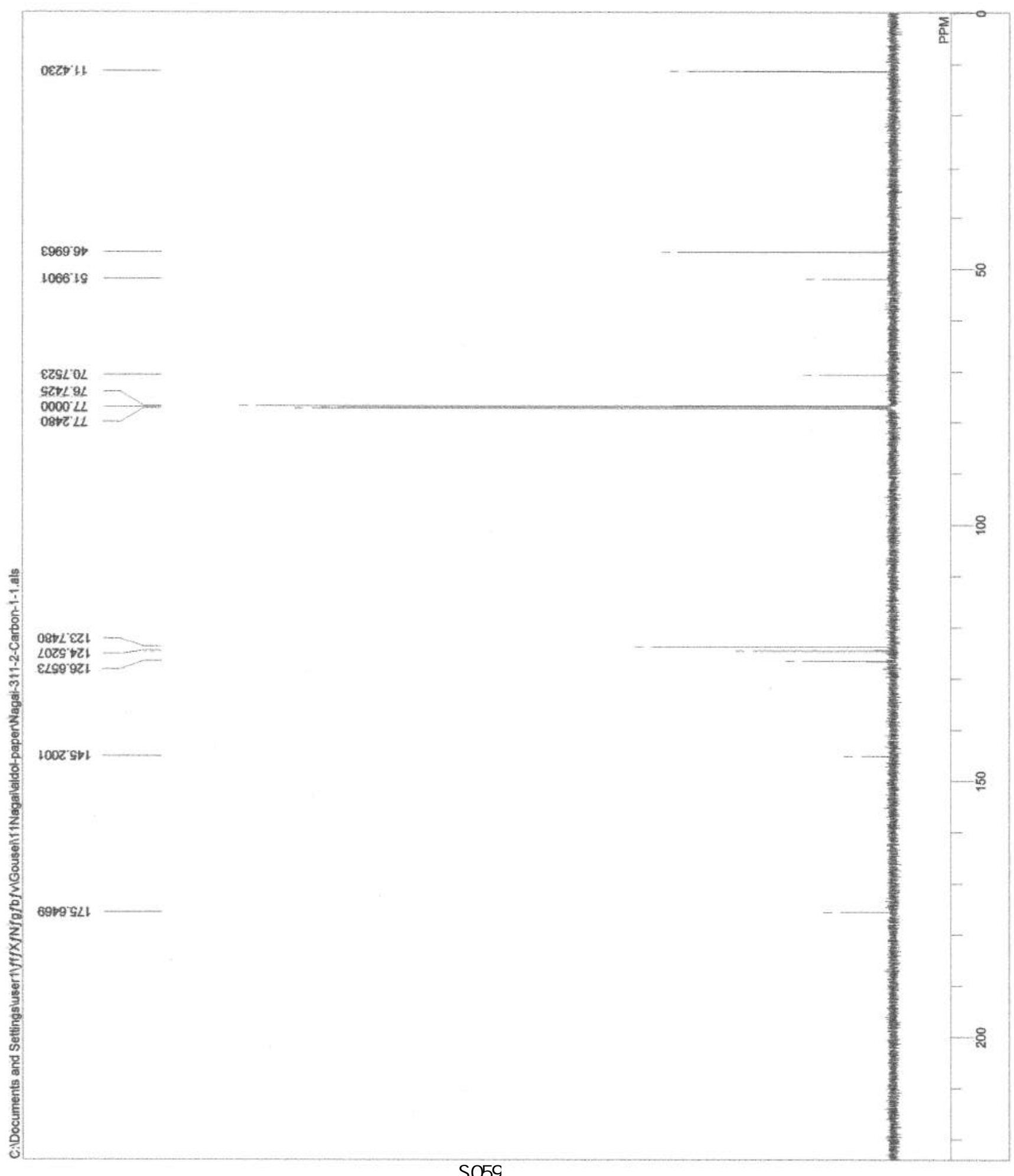



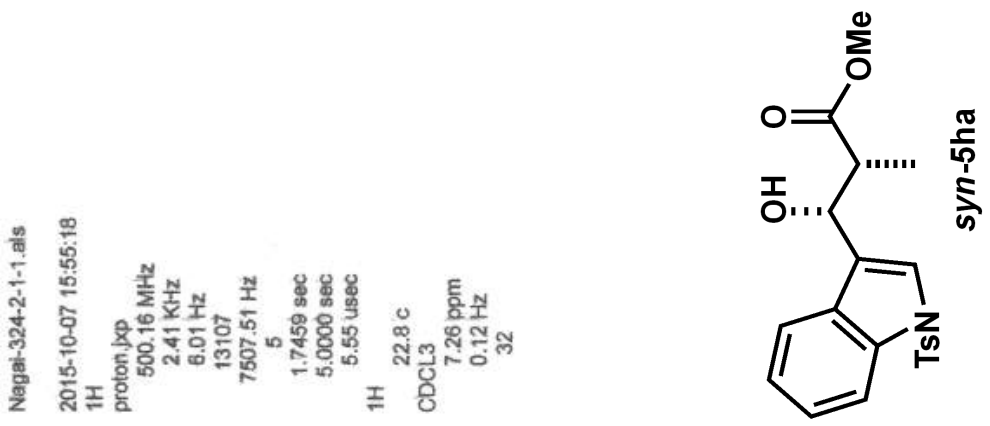

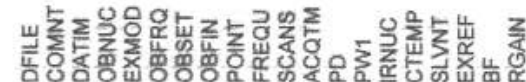

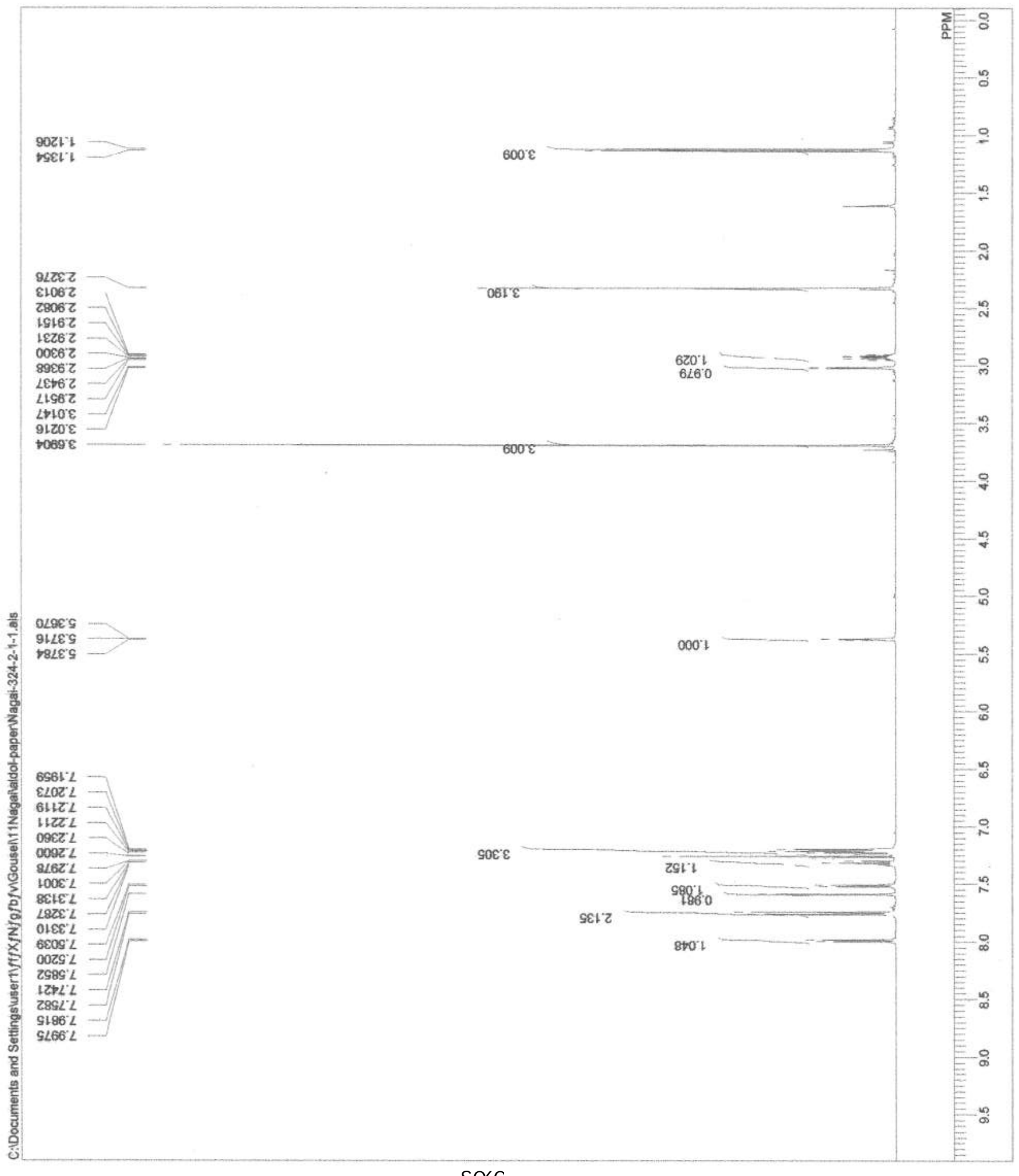



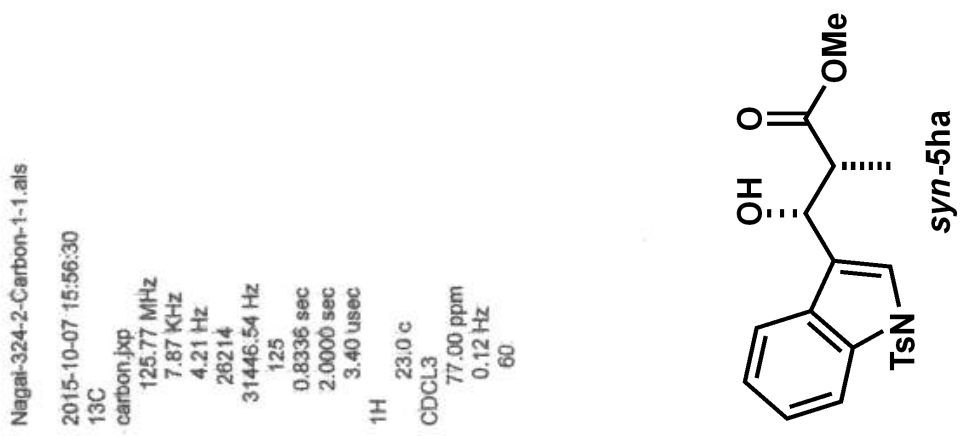

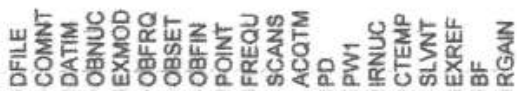

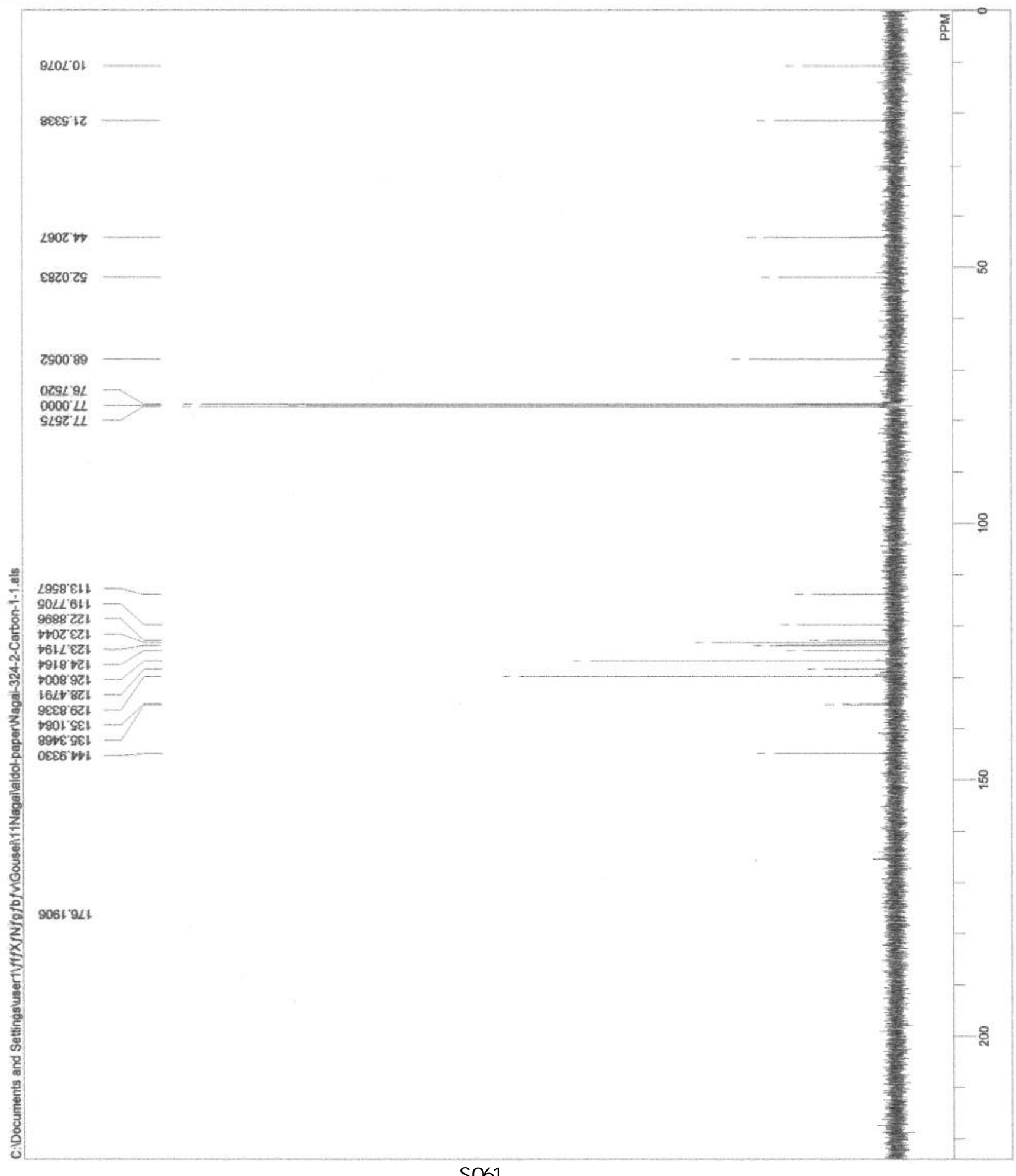



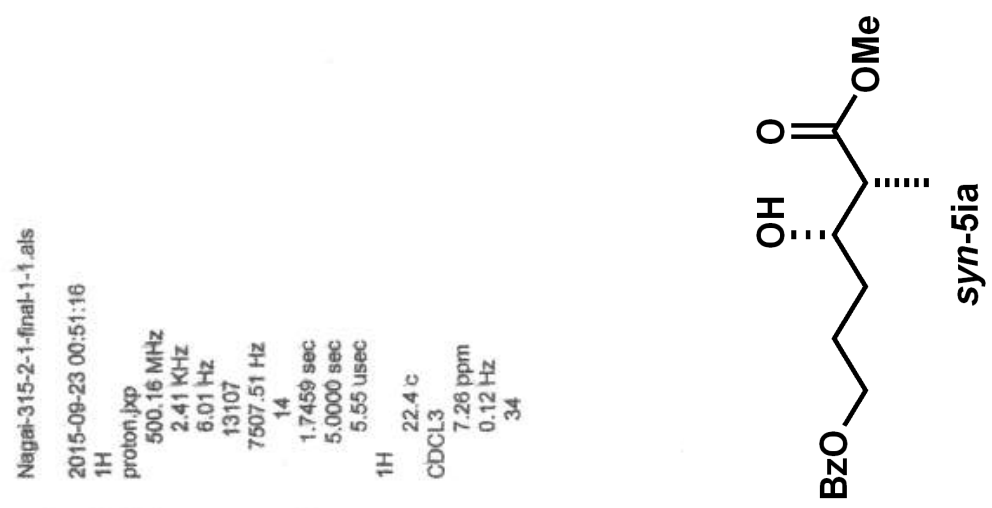

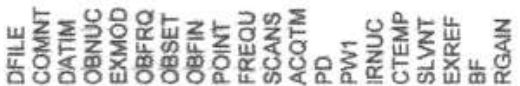

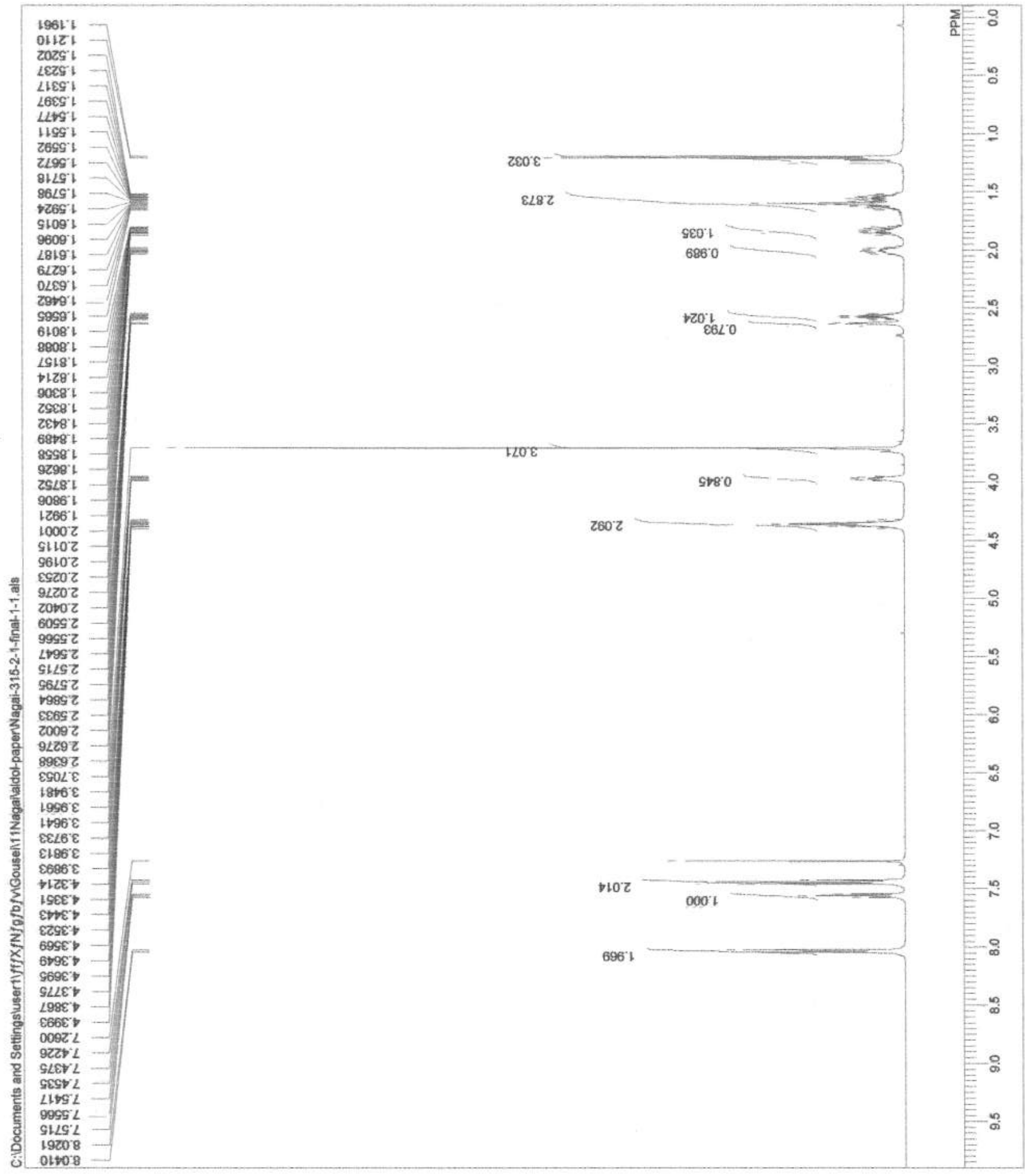



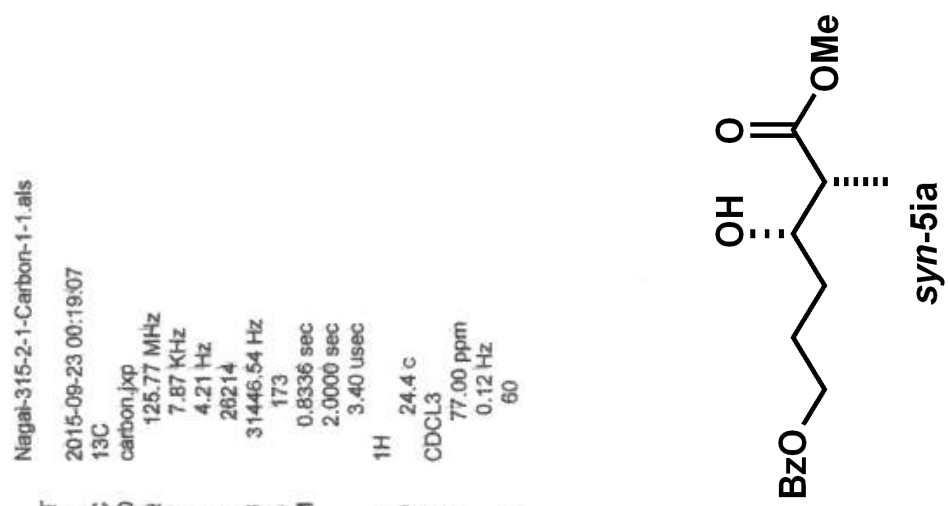

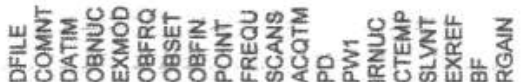

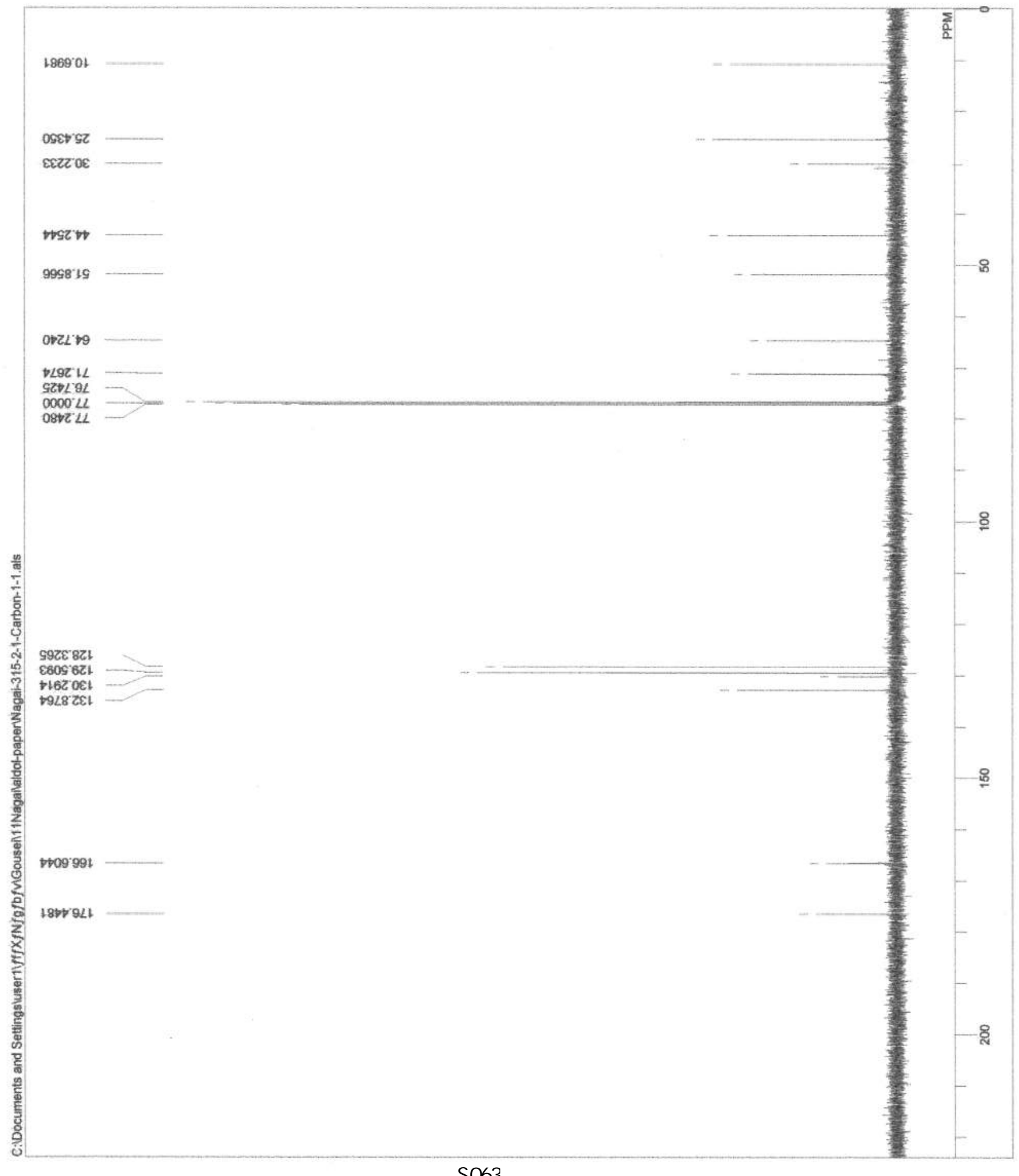




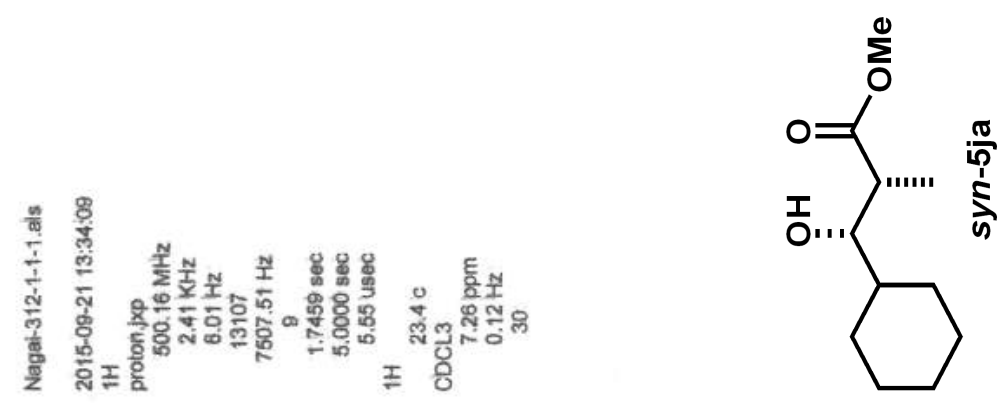

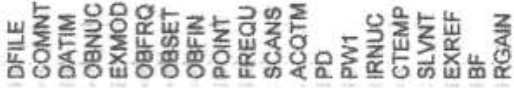

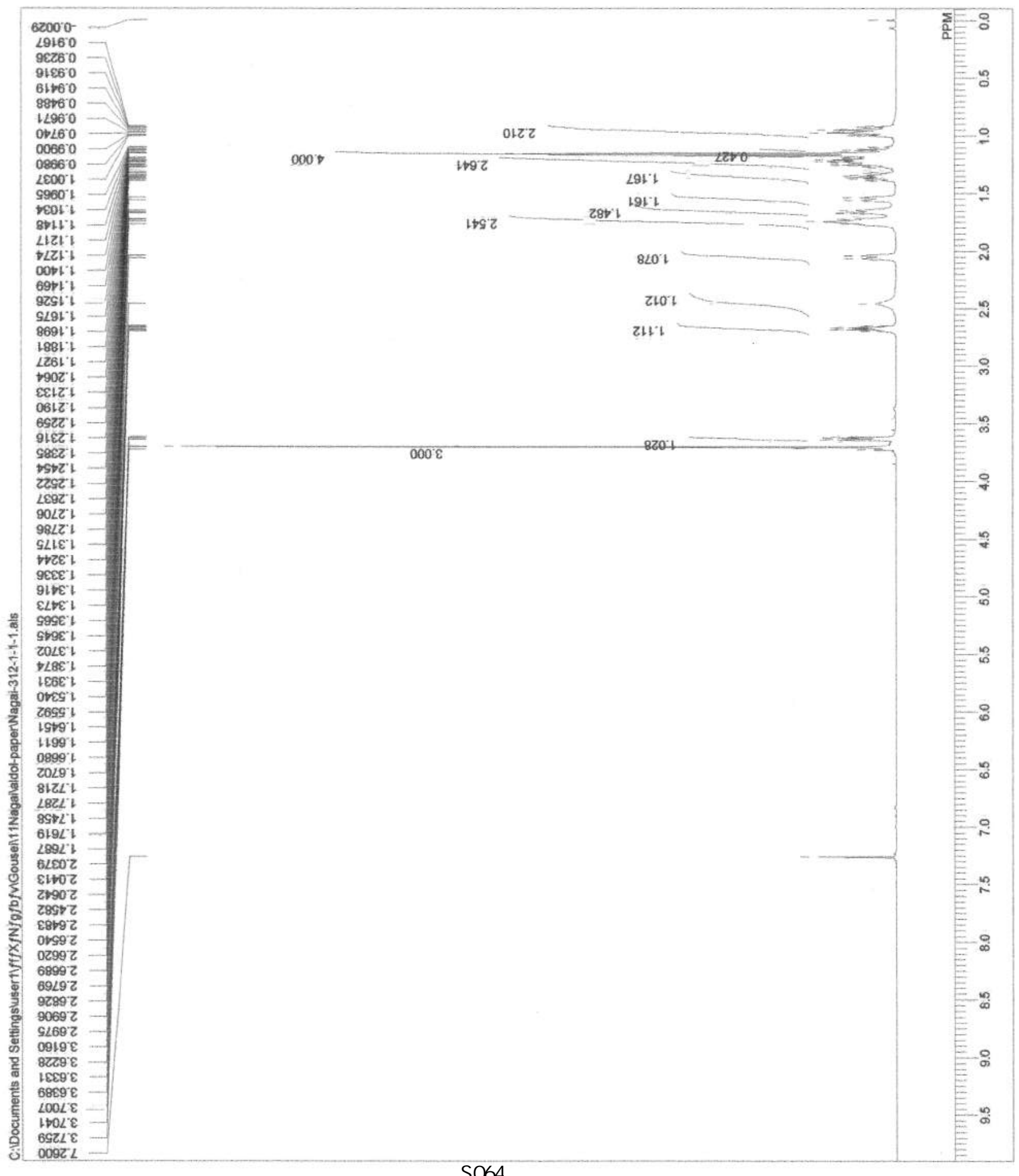



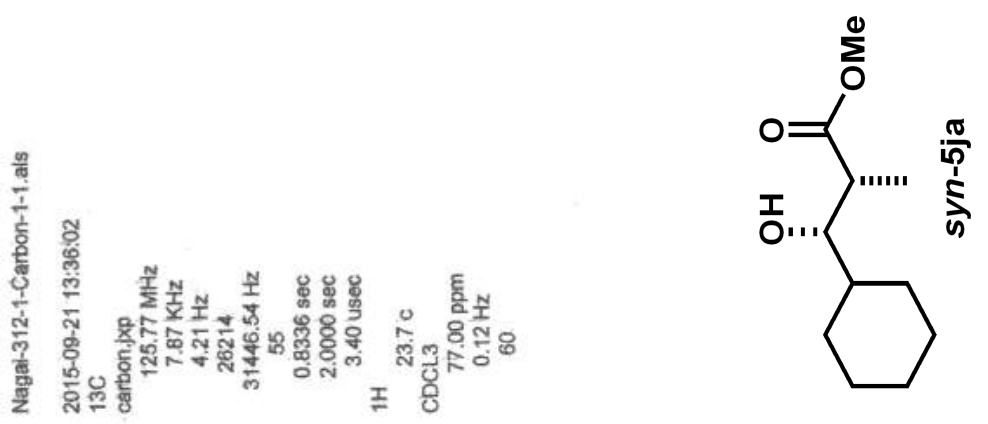

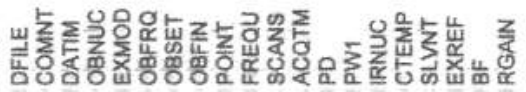

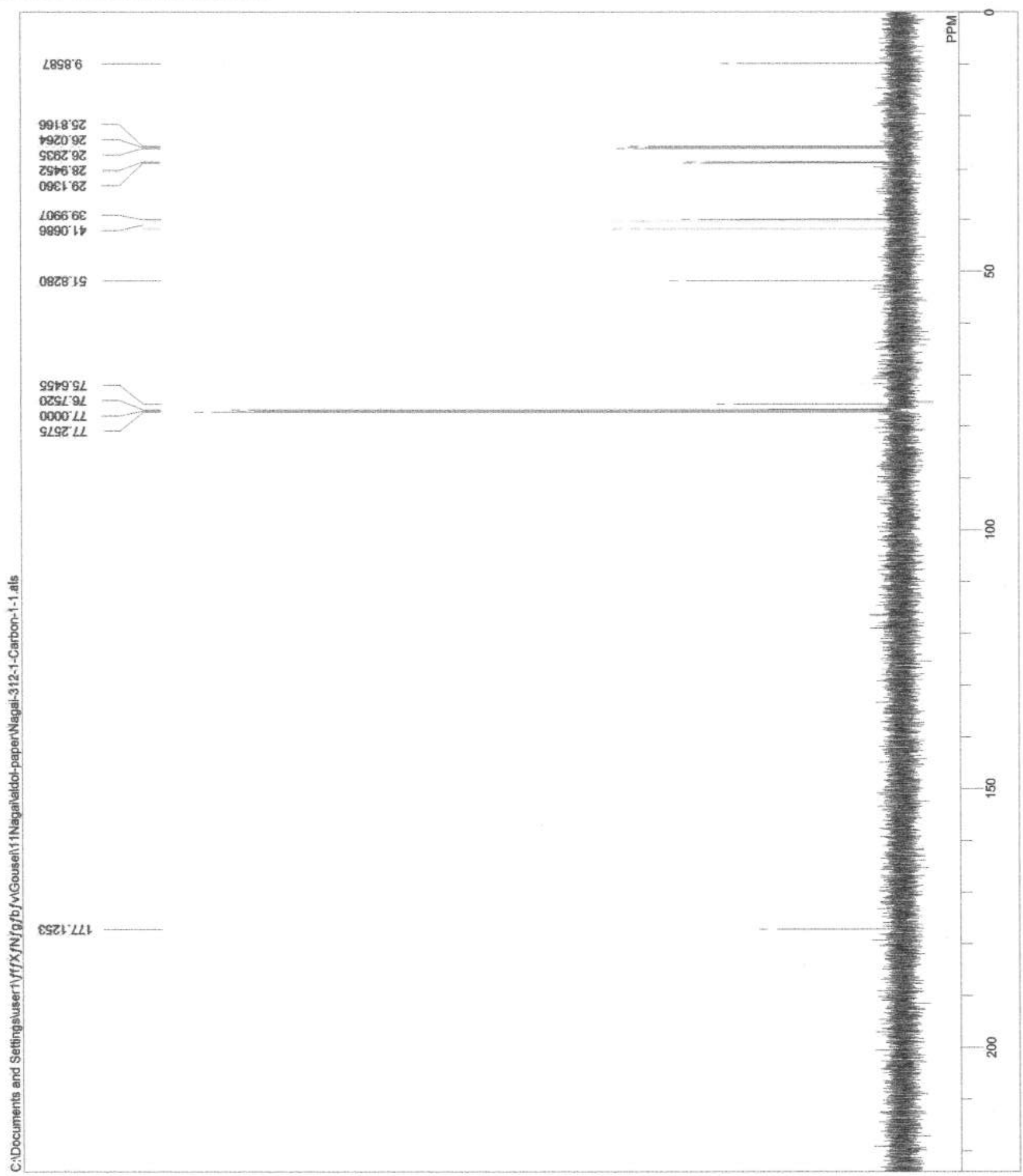




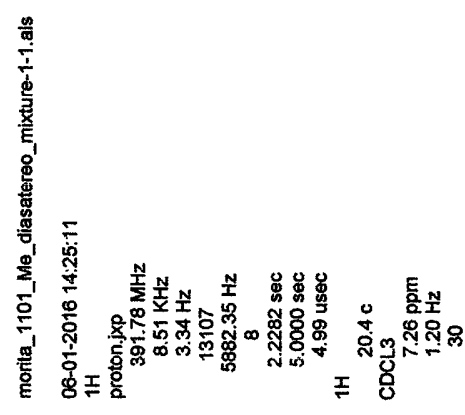

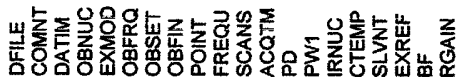
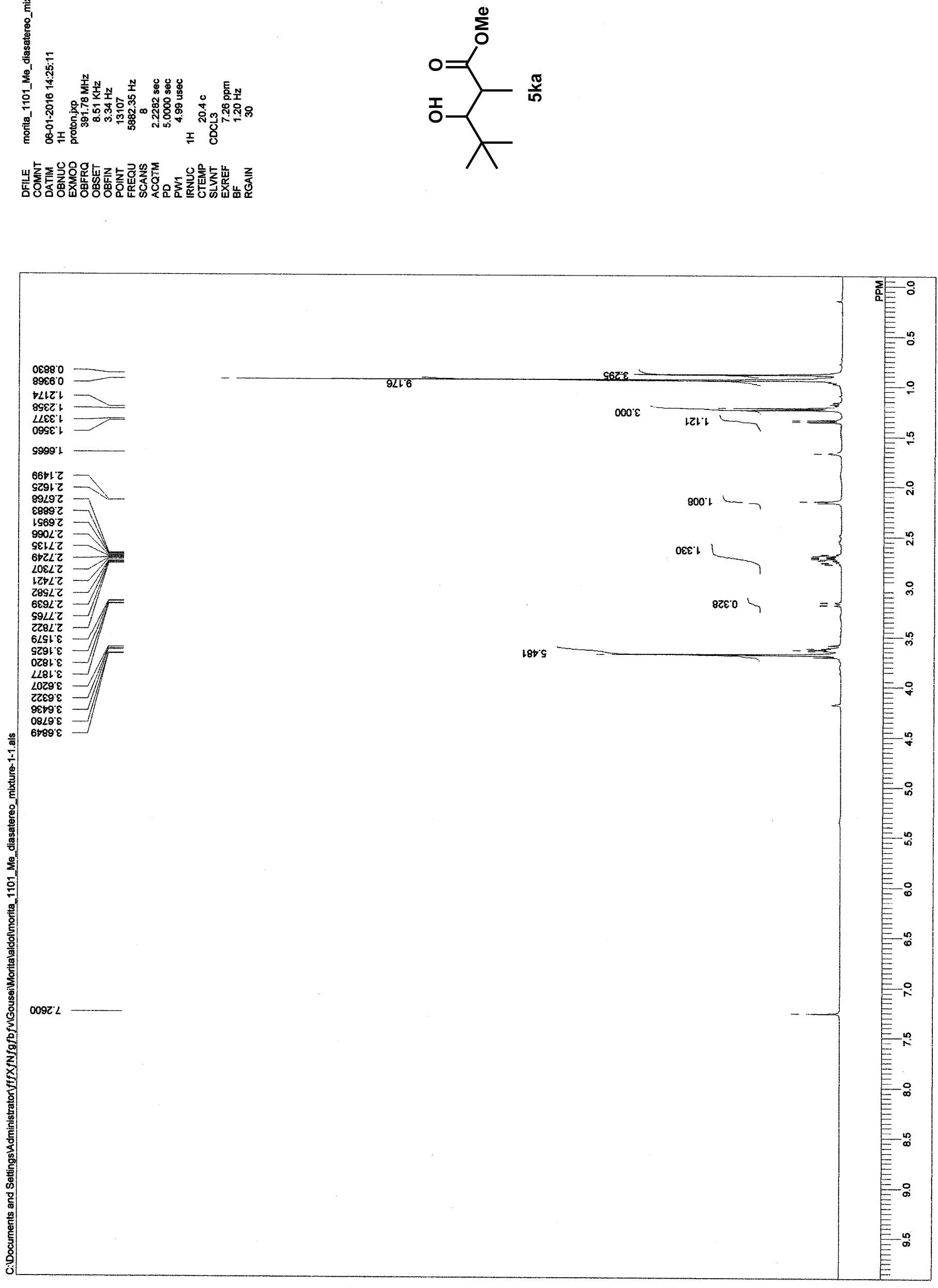


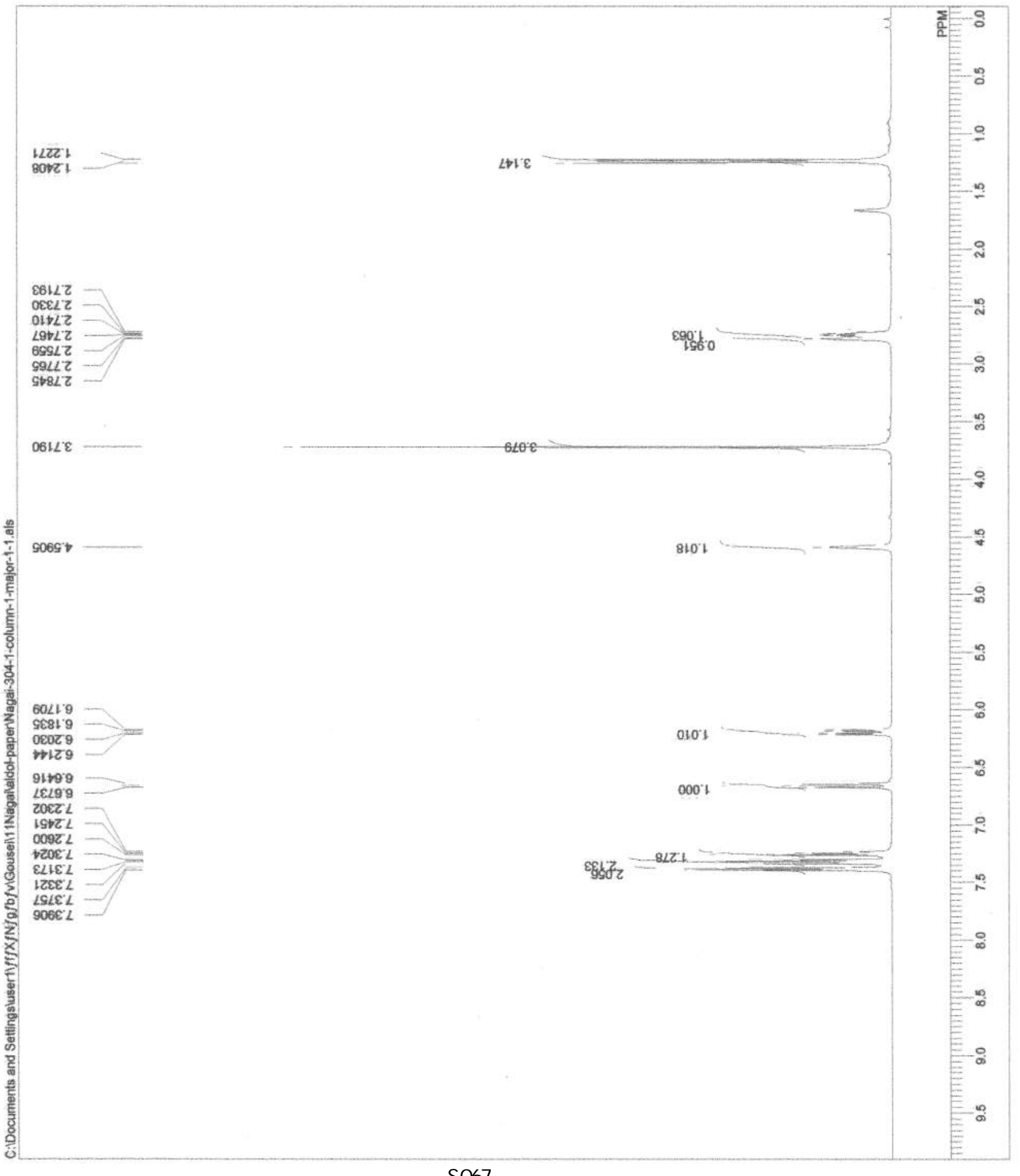


oвz'।

$\$ \nabla 28 \%$

L568\S

$8 \varepsilon 662 L$

$920+L: 9 L$

$00002 L$

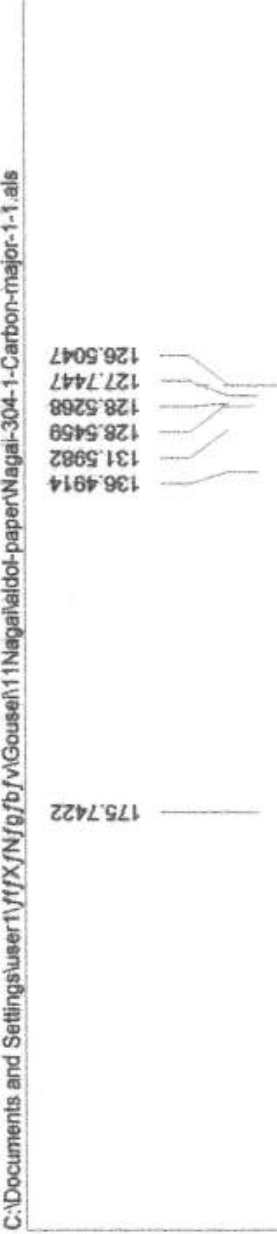



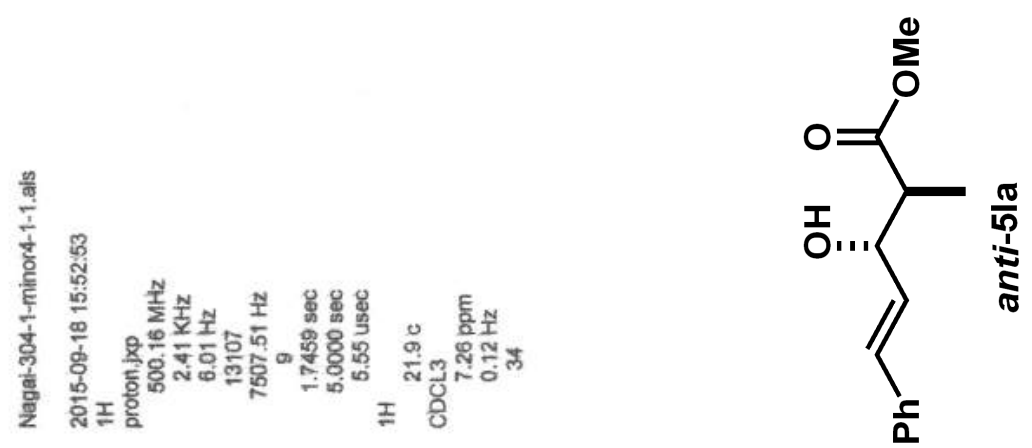

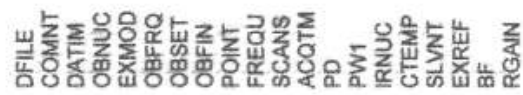

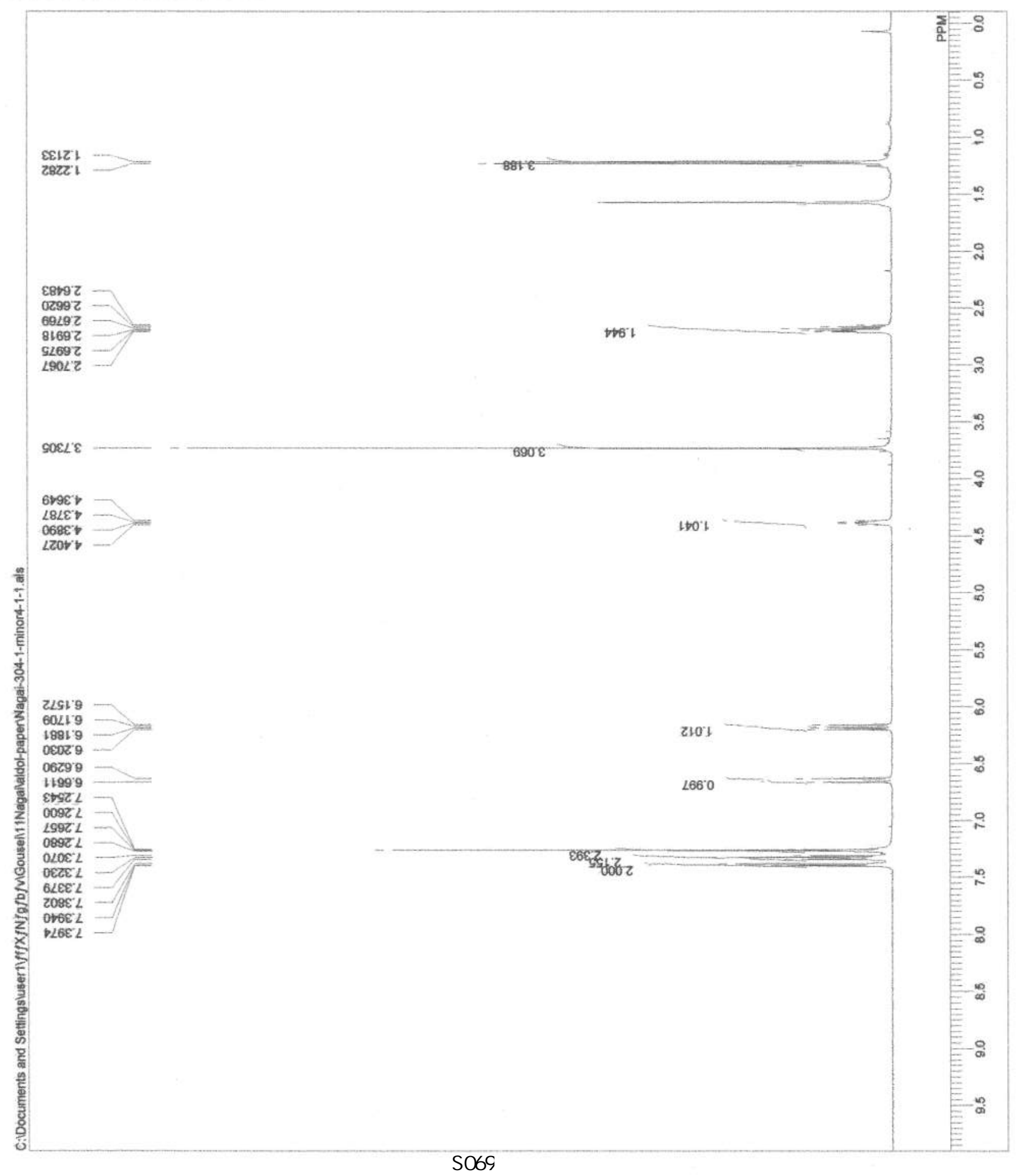



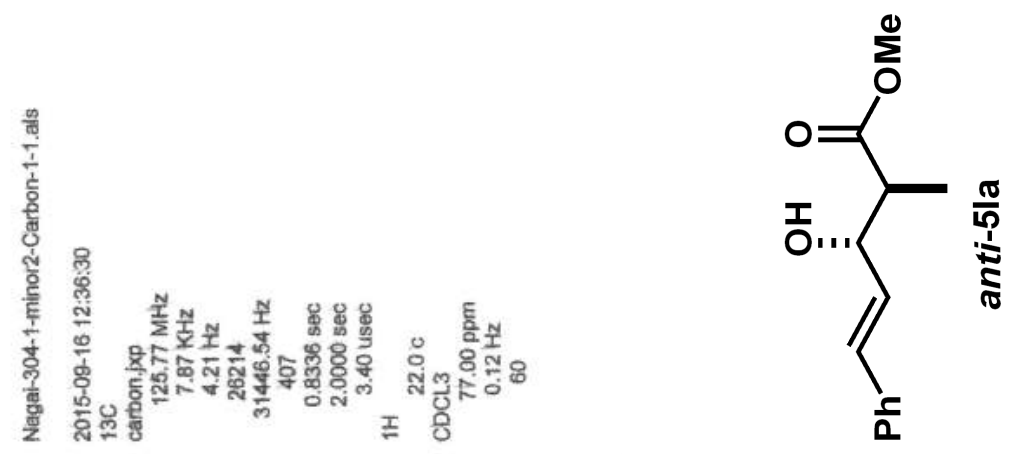

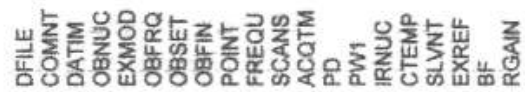

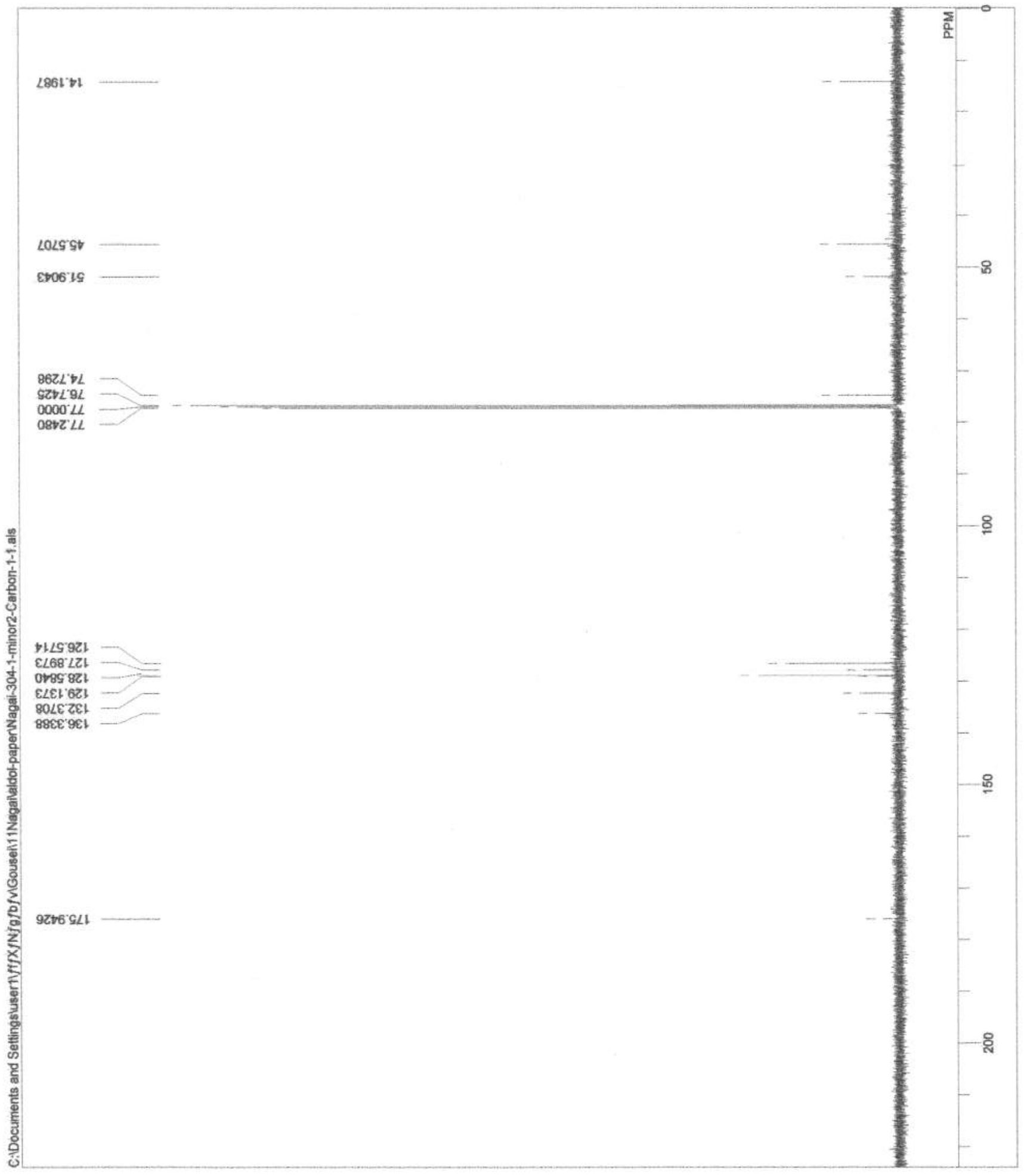




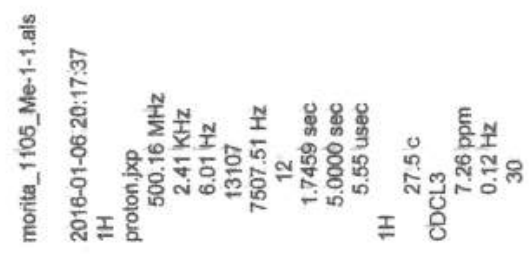

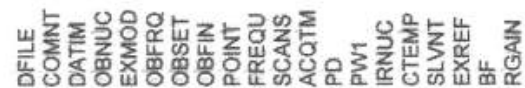
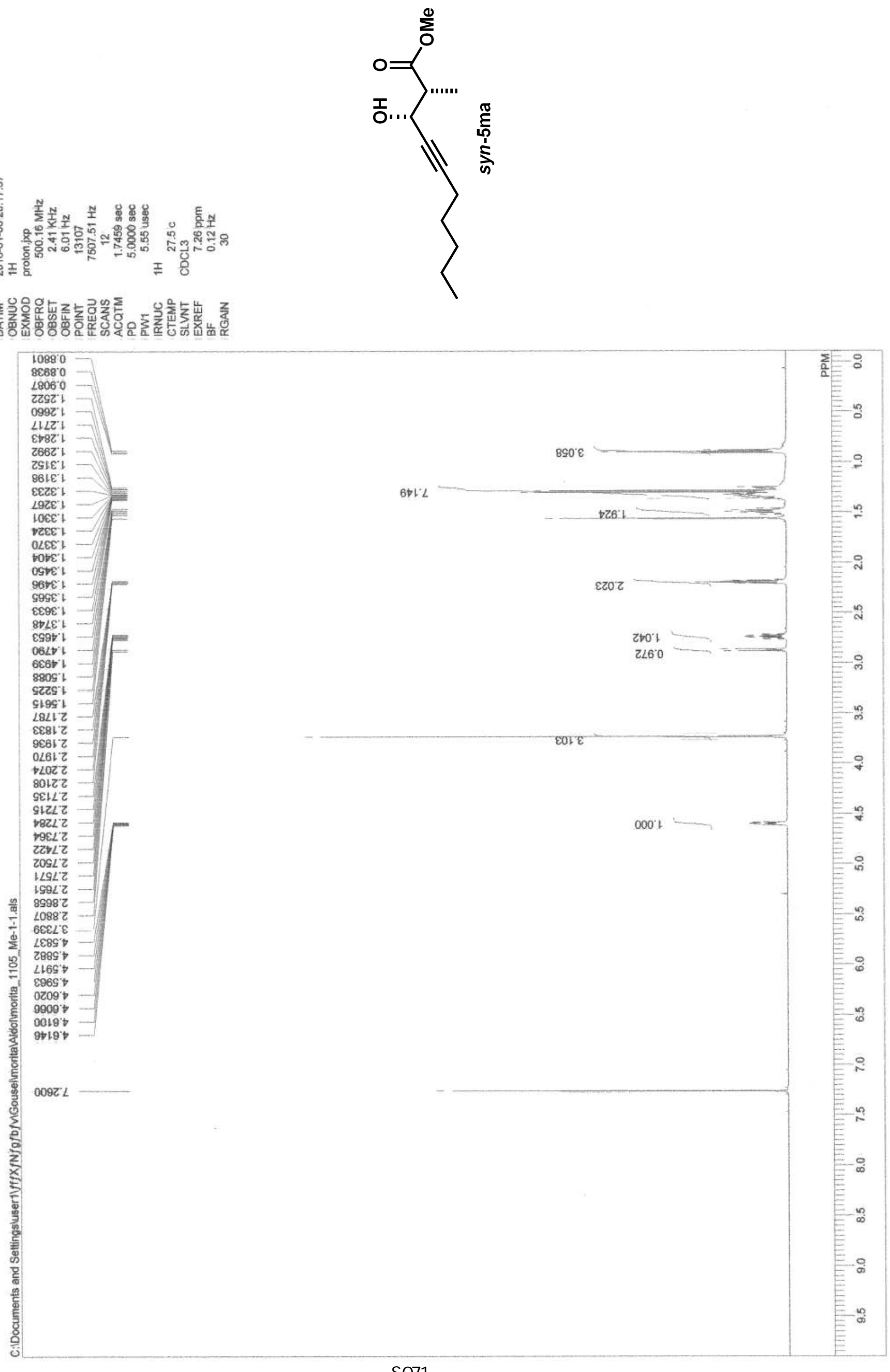

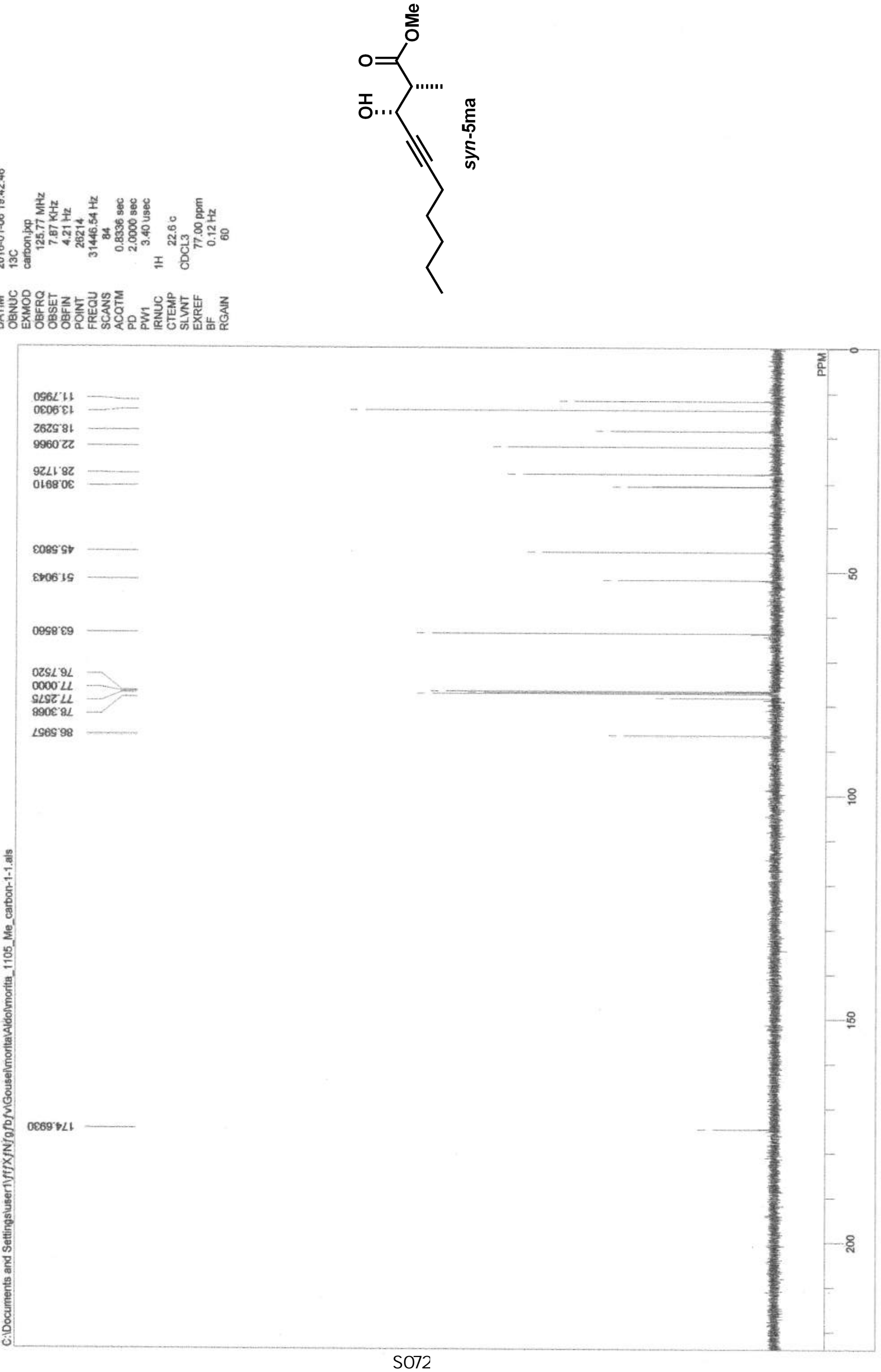

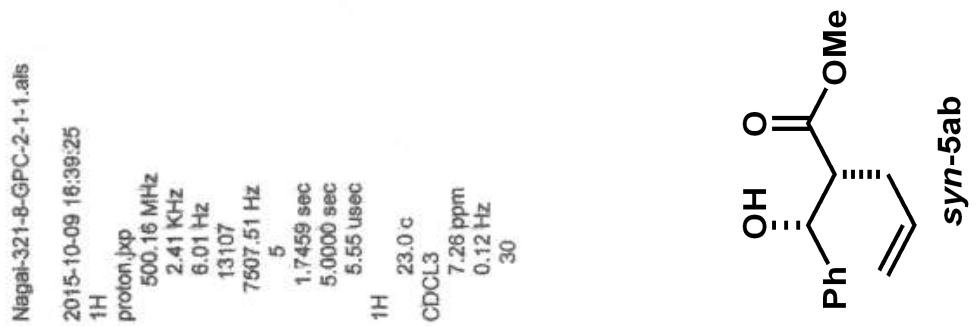

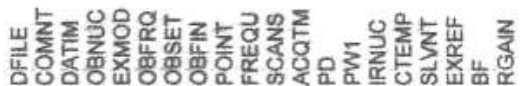

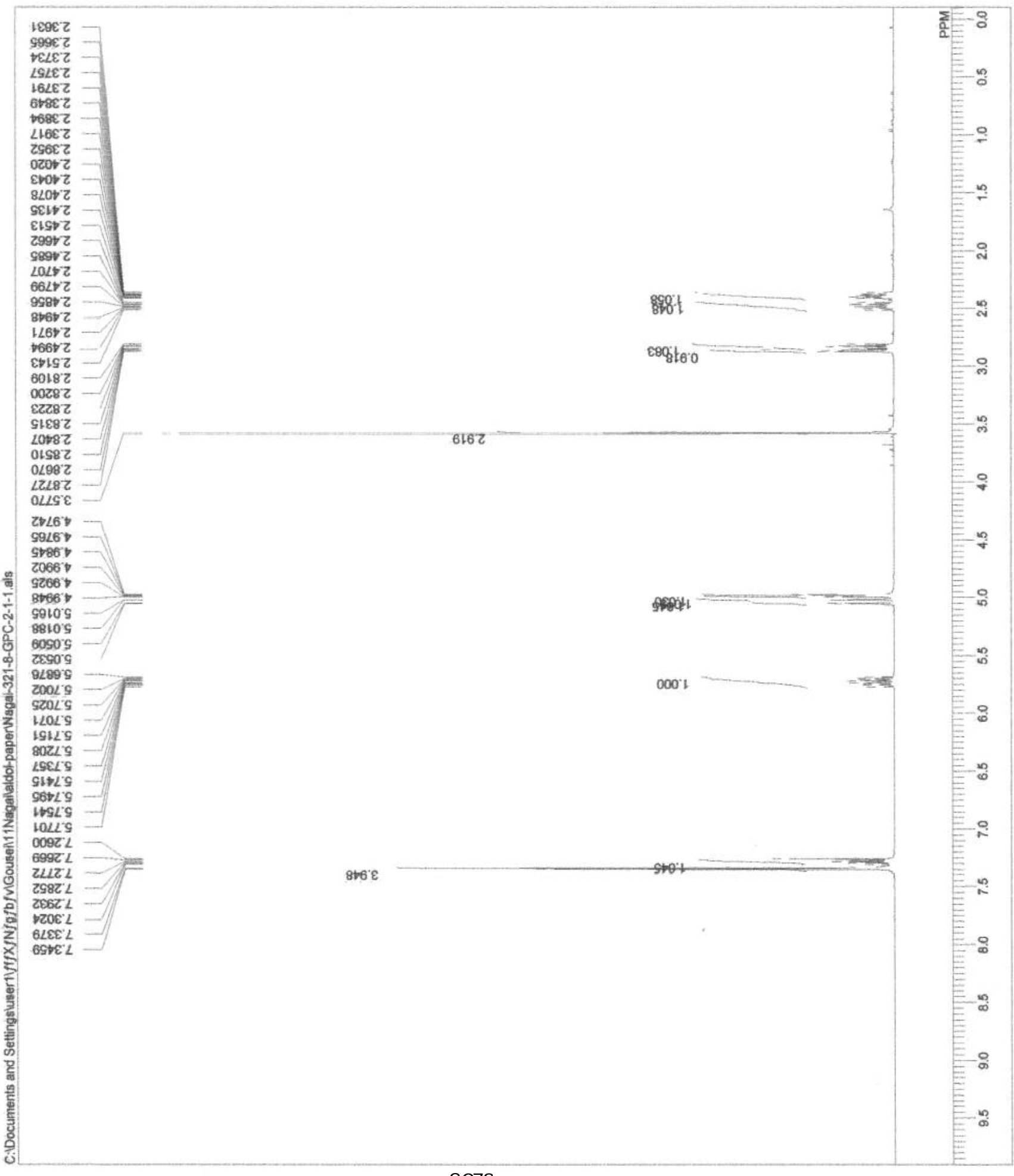



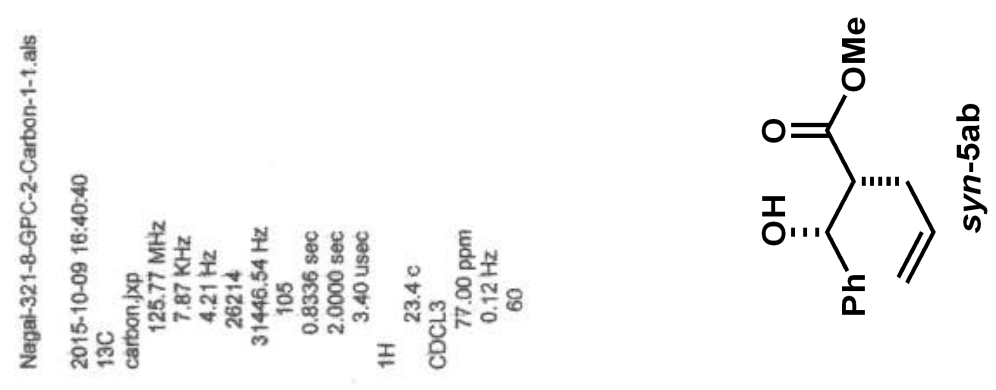

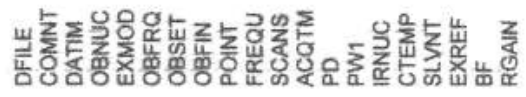

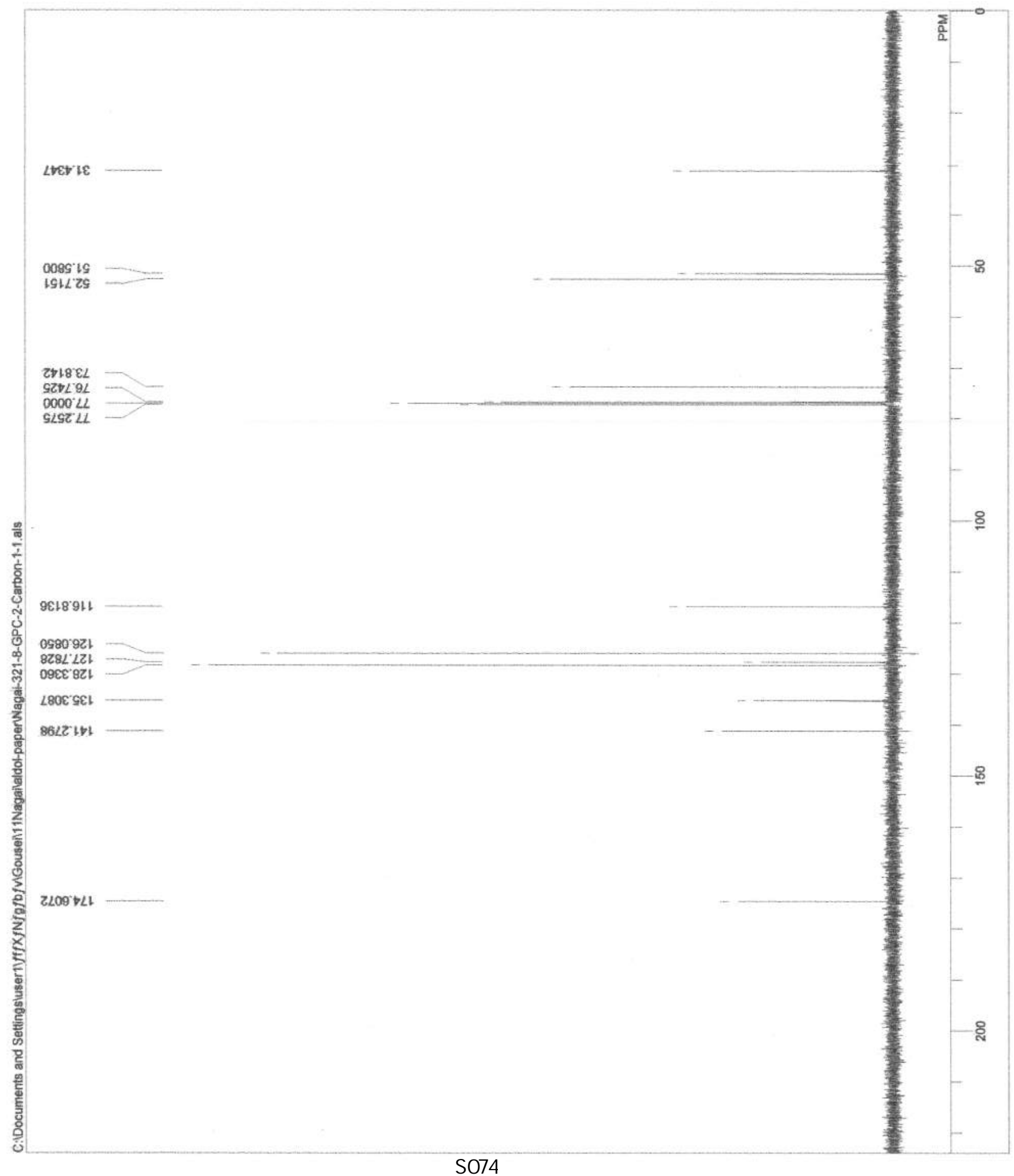



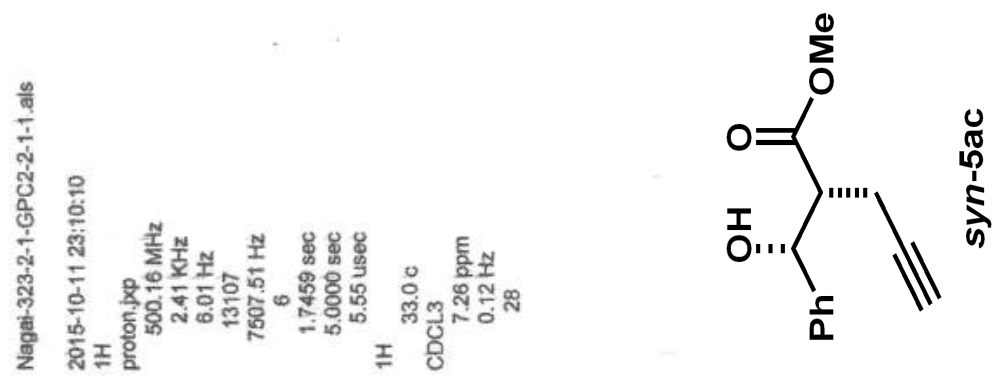

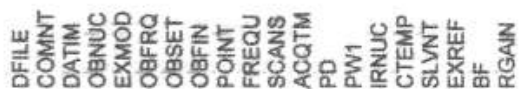

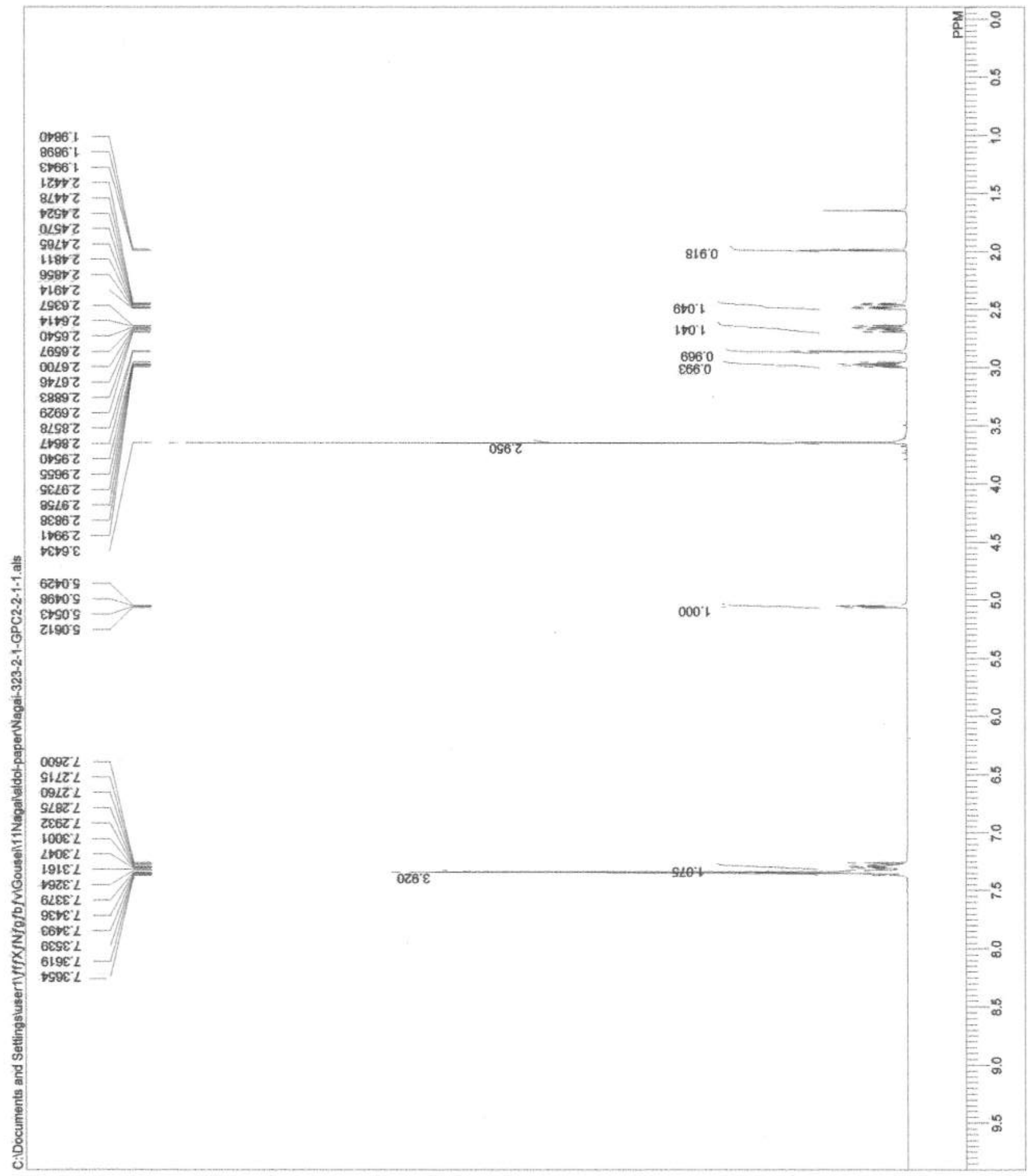



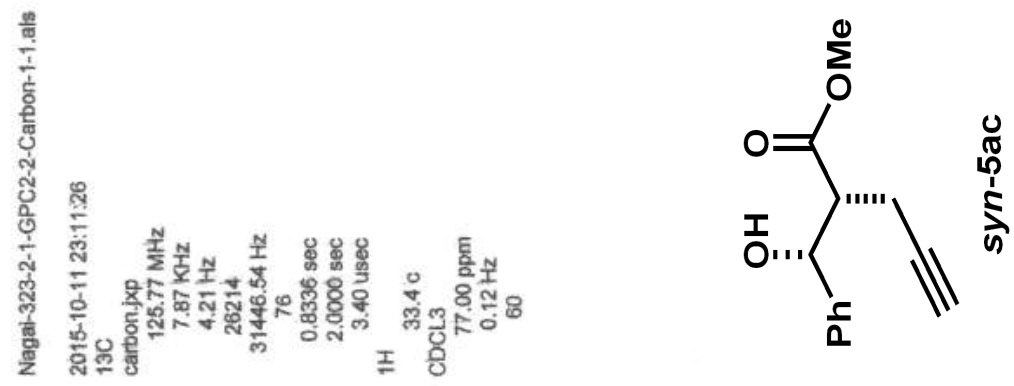

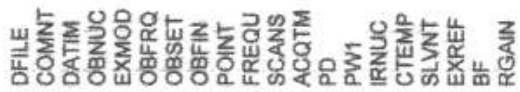

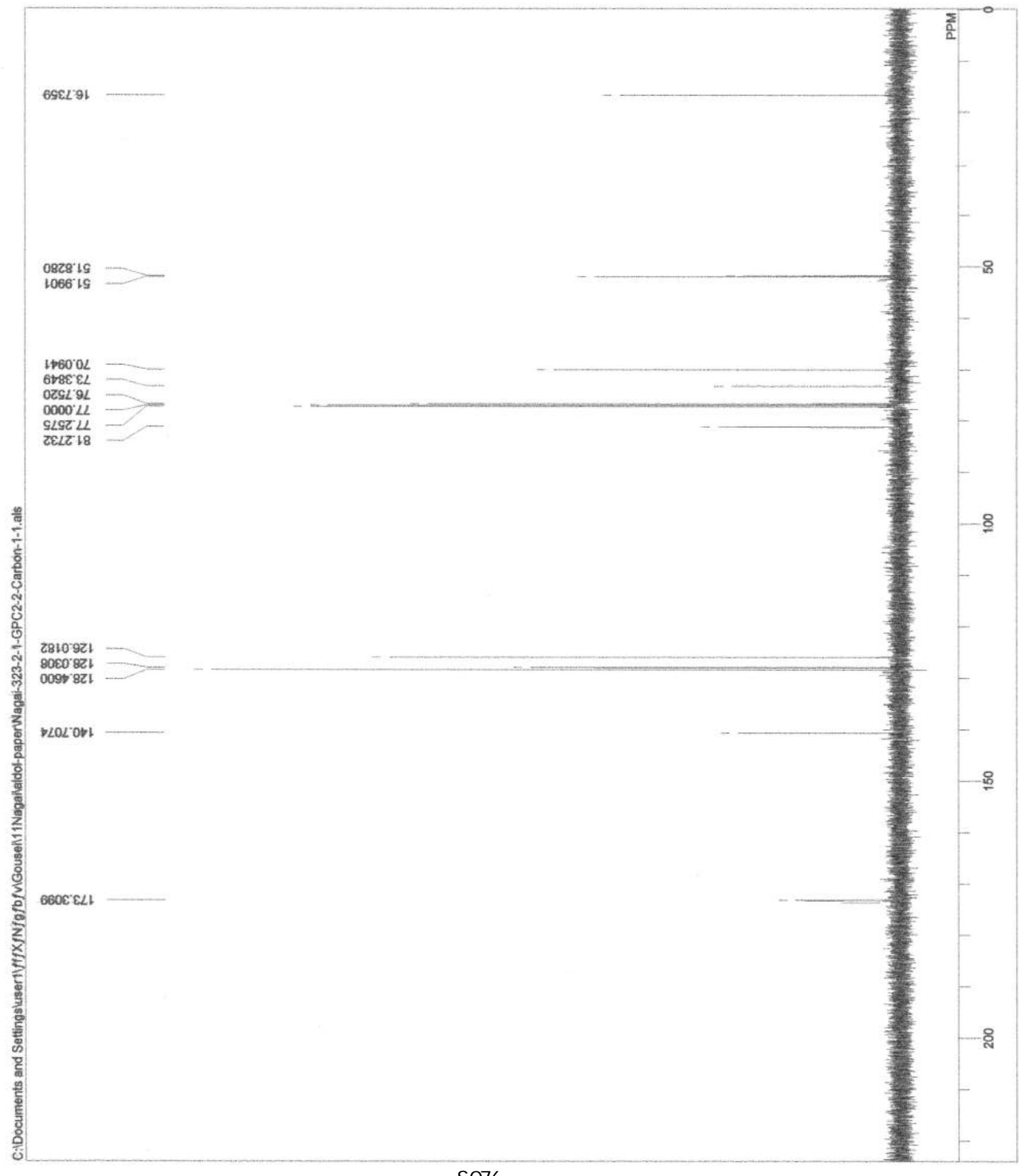



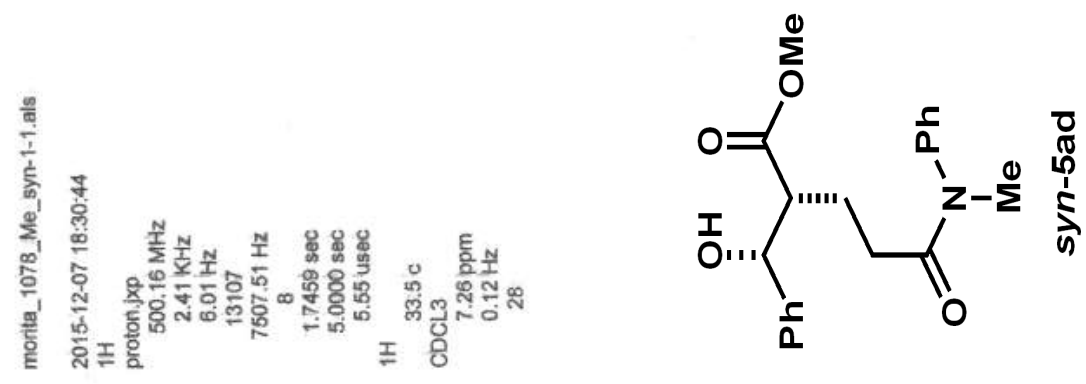

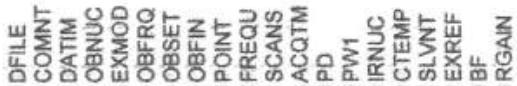

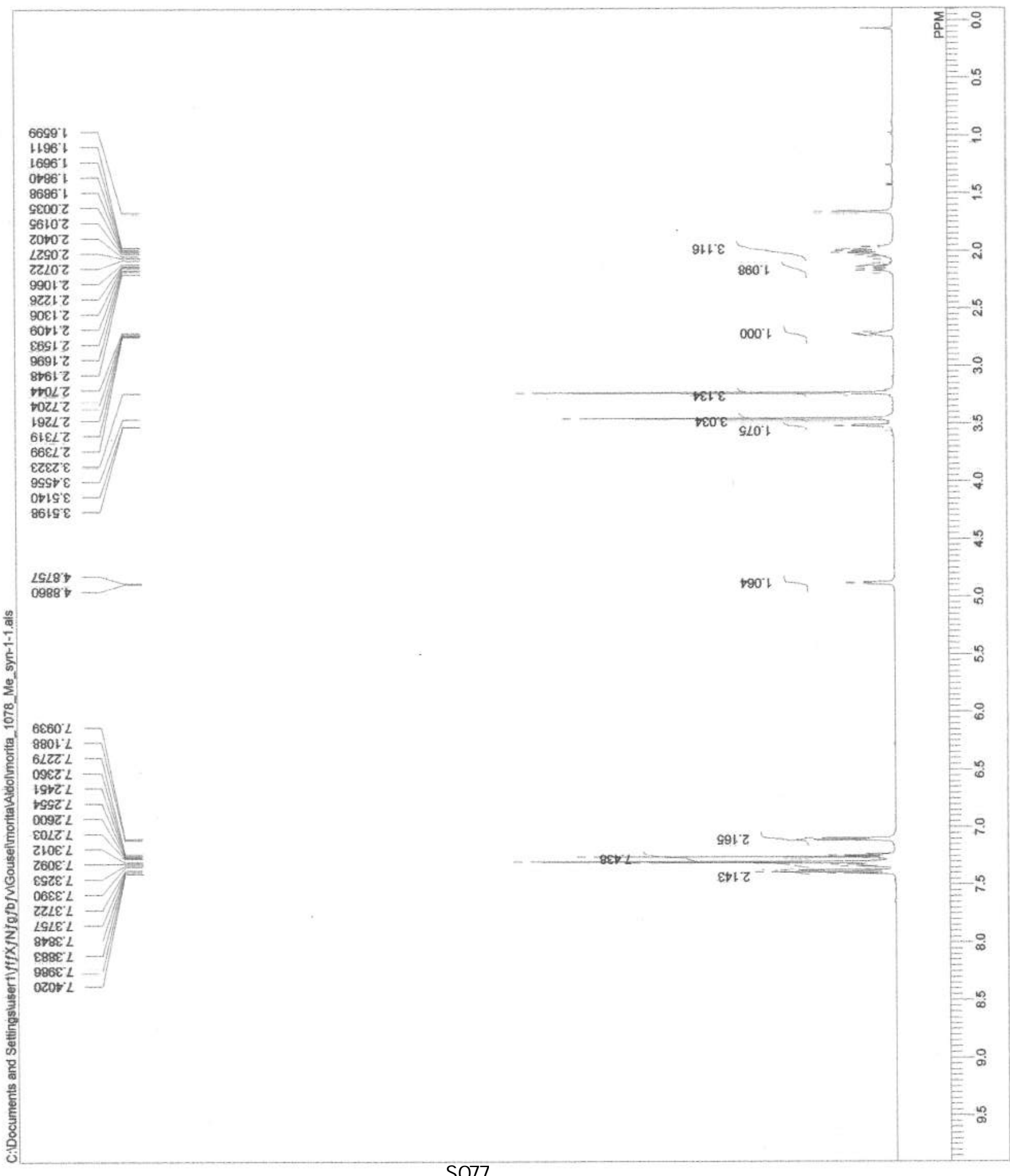



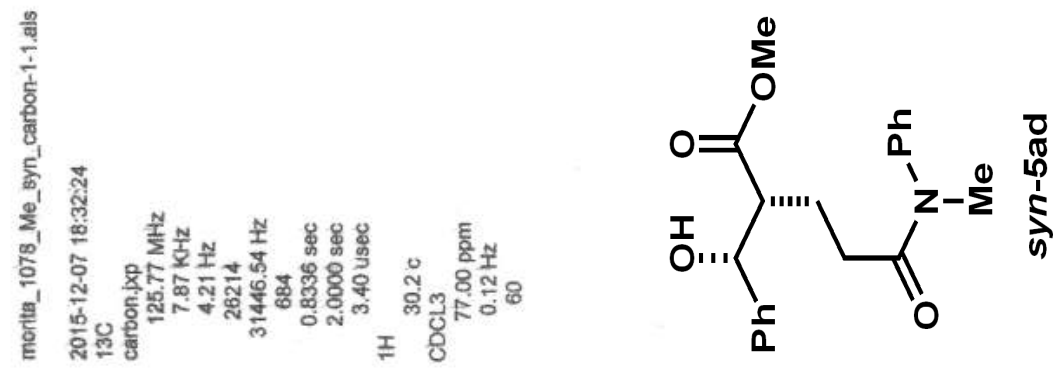

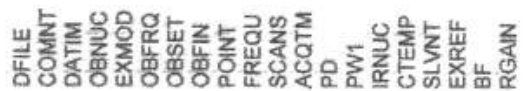

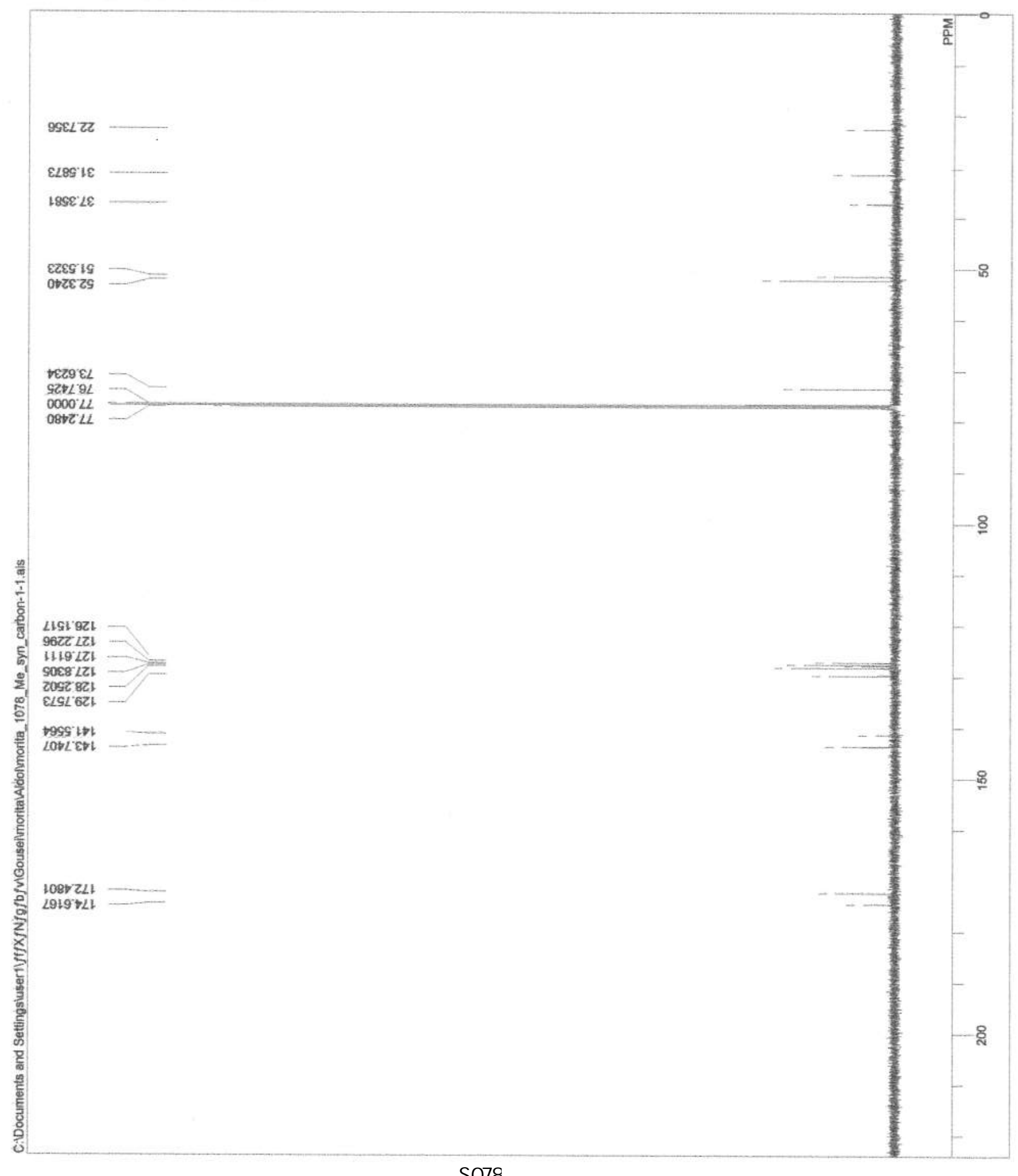



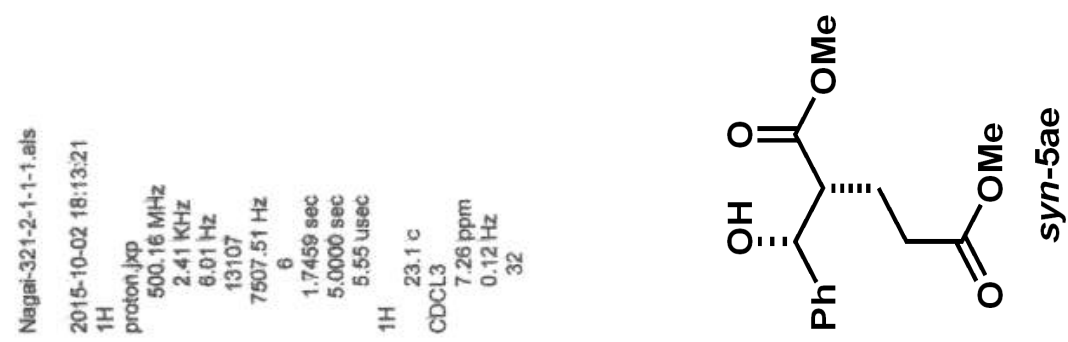

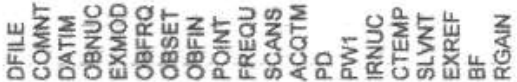

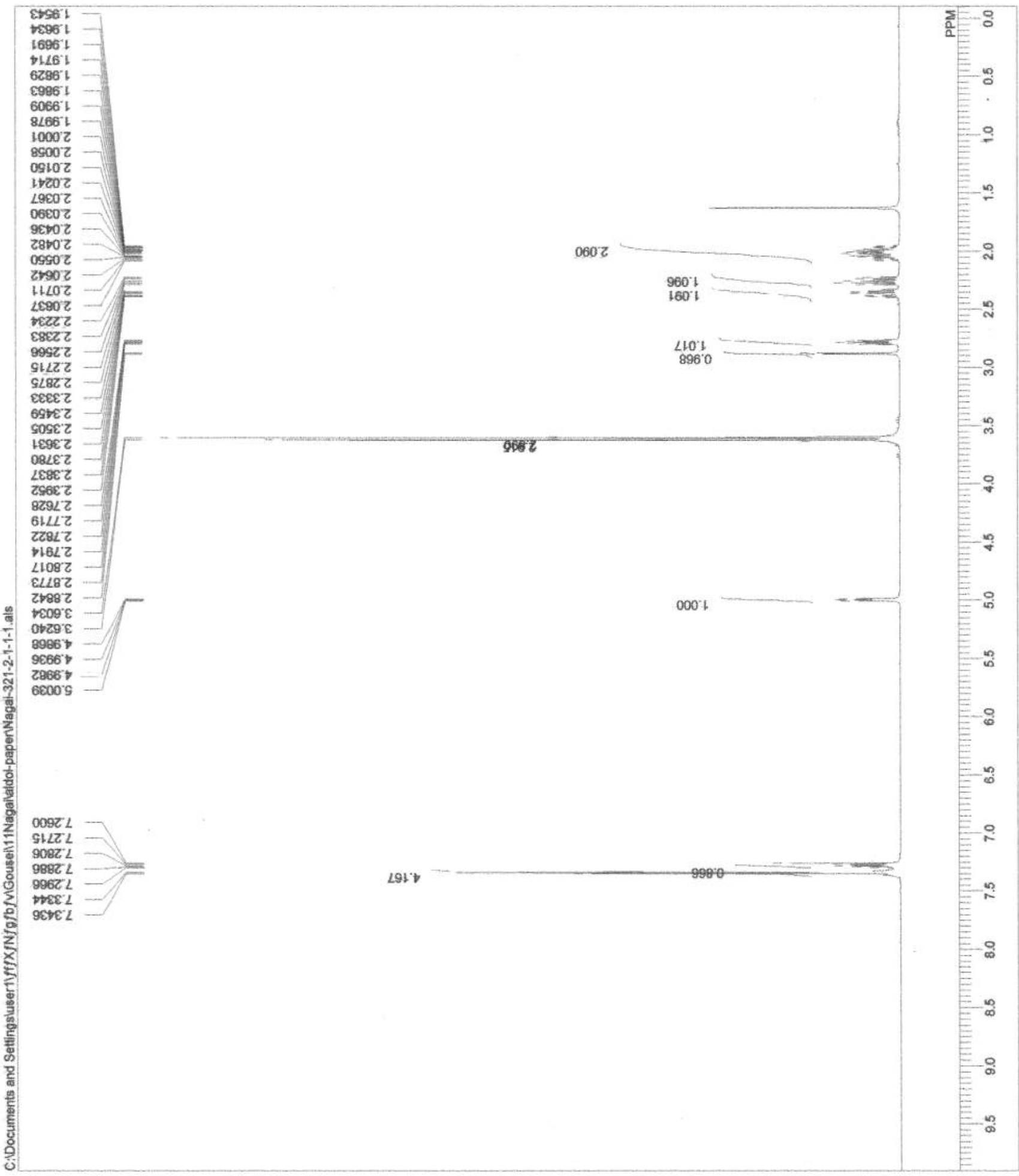



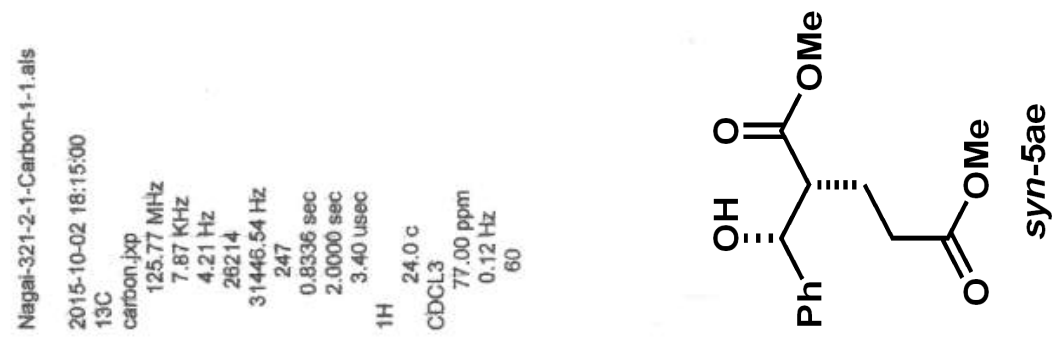

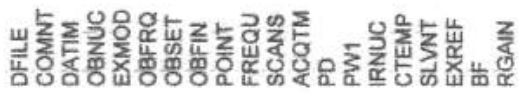

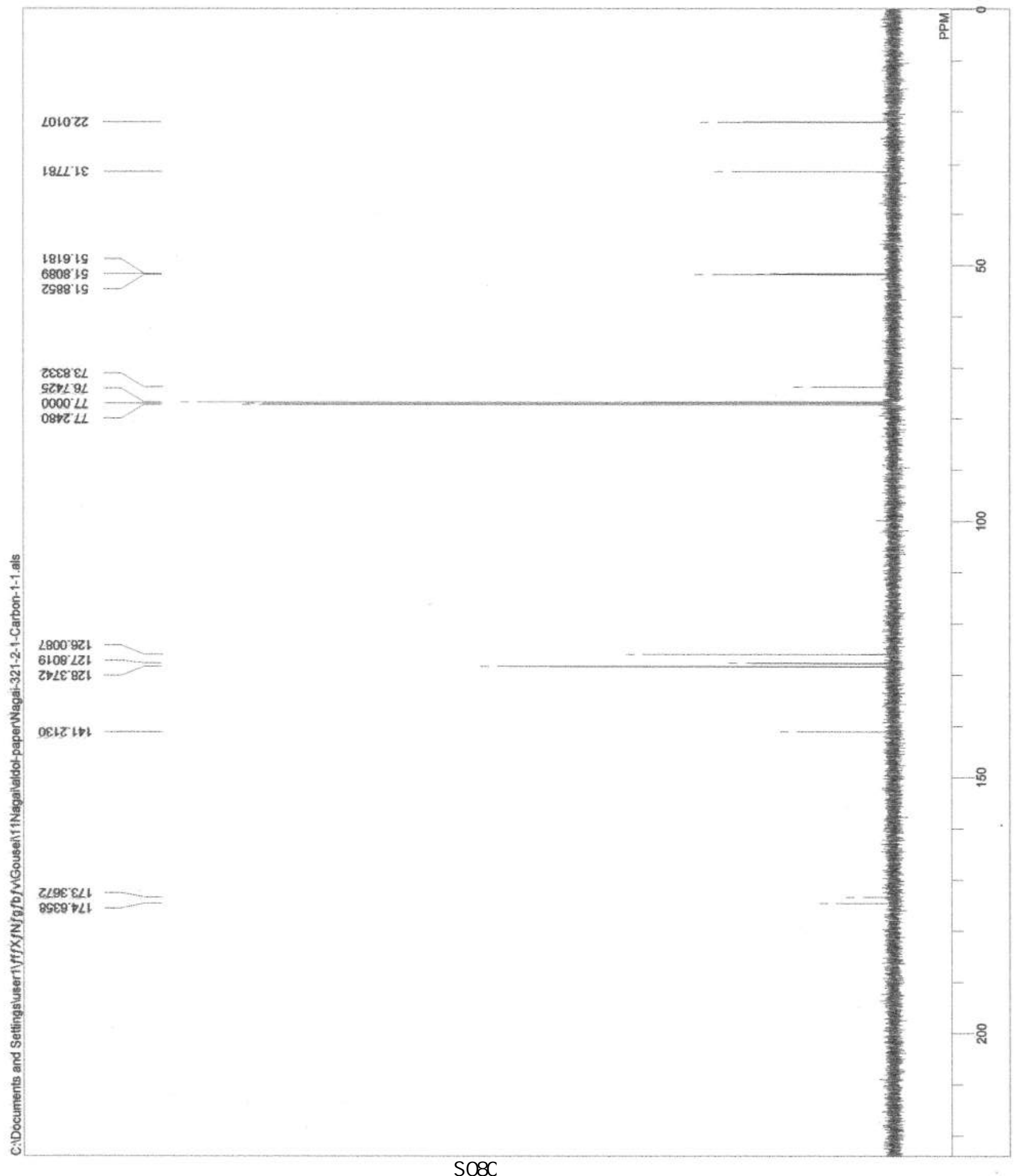



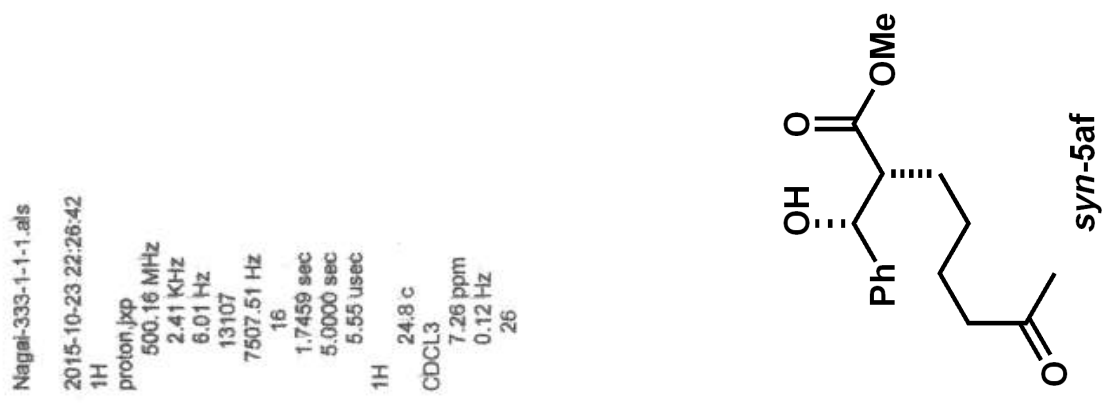

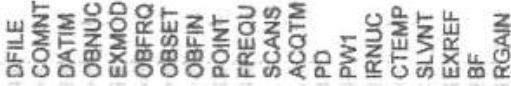

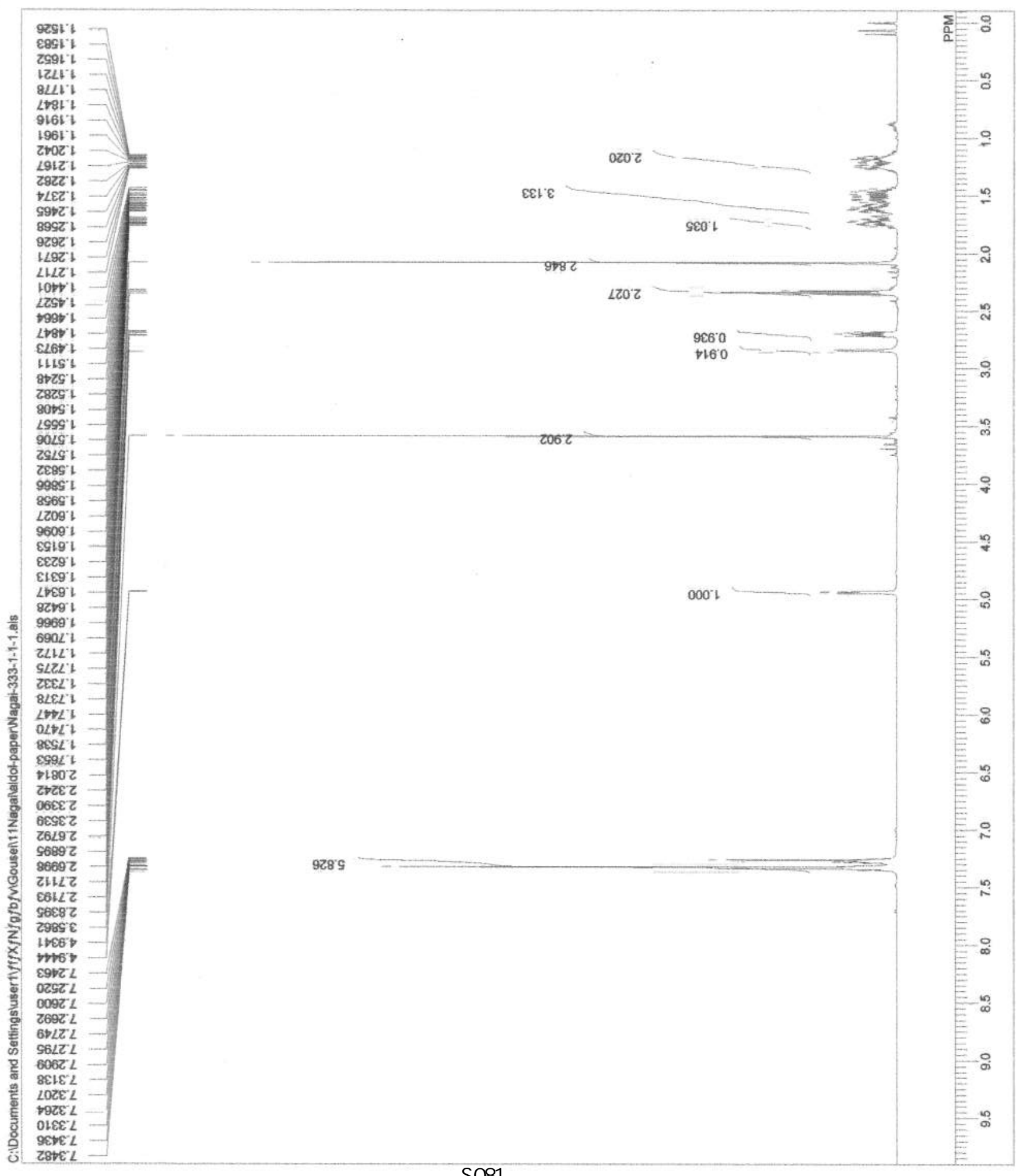



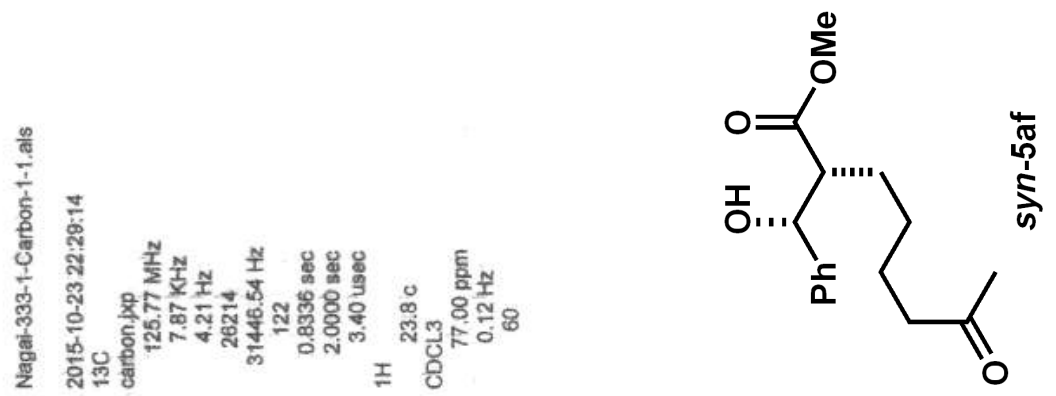

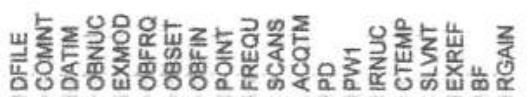

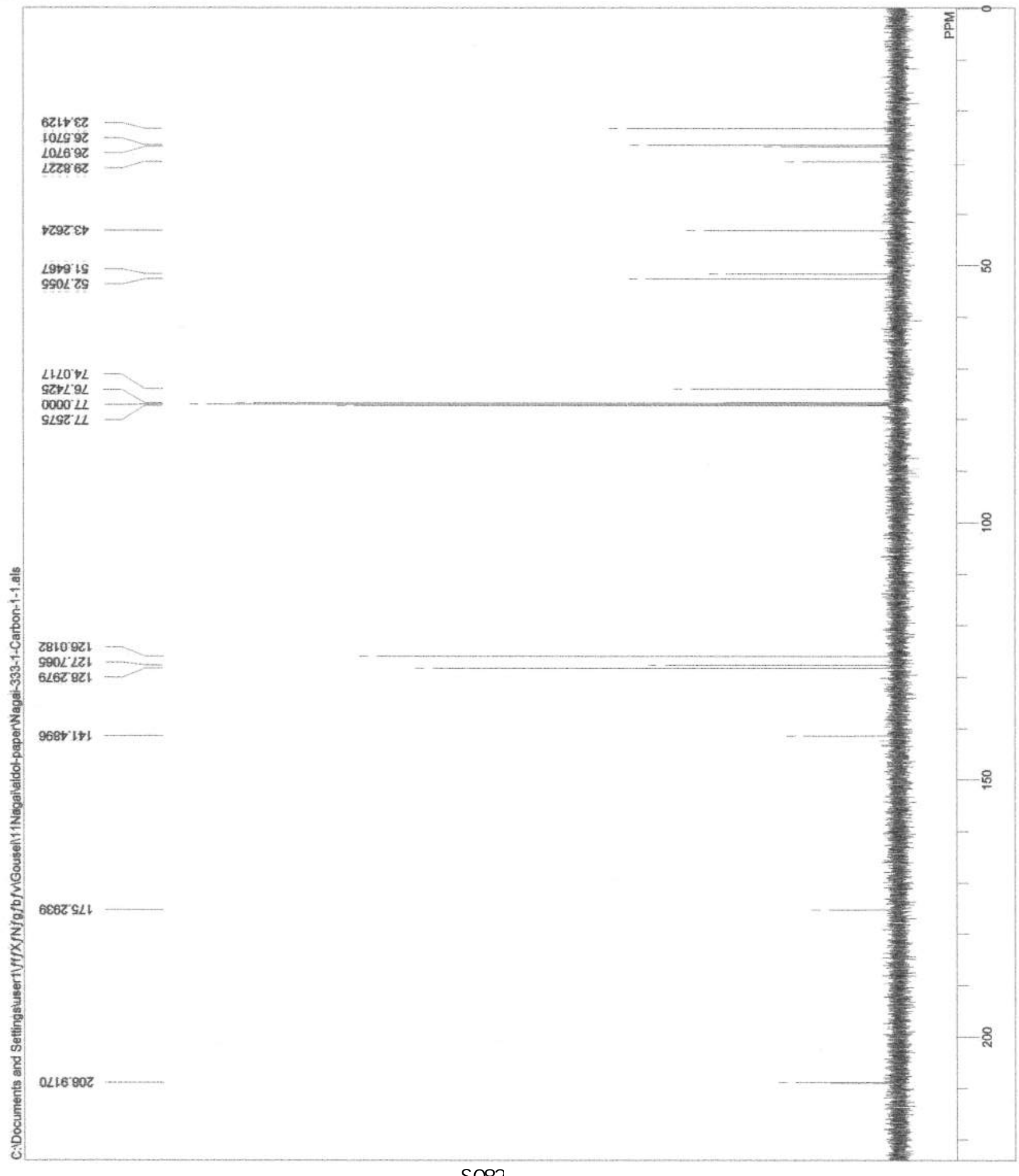



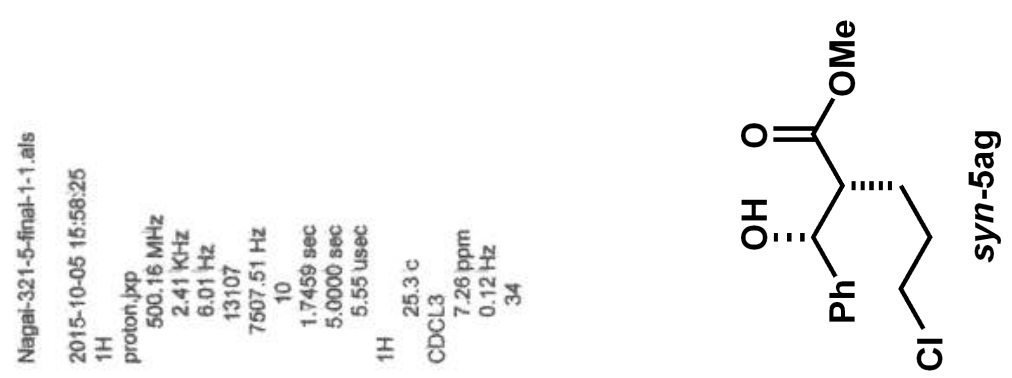

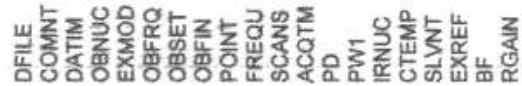

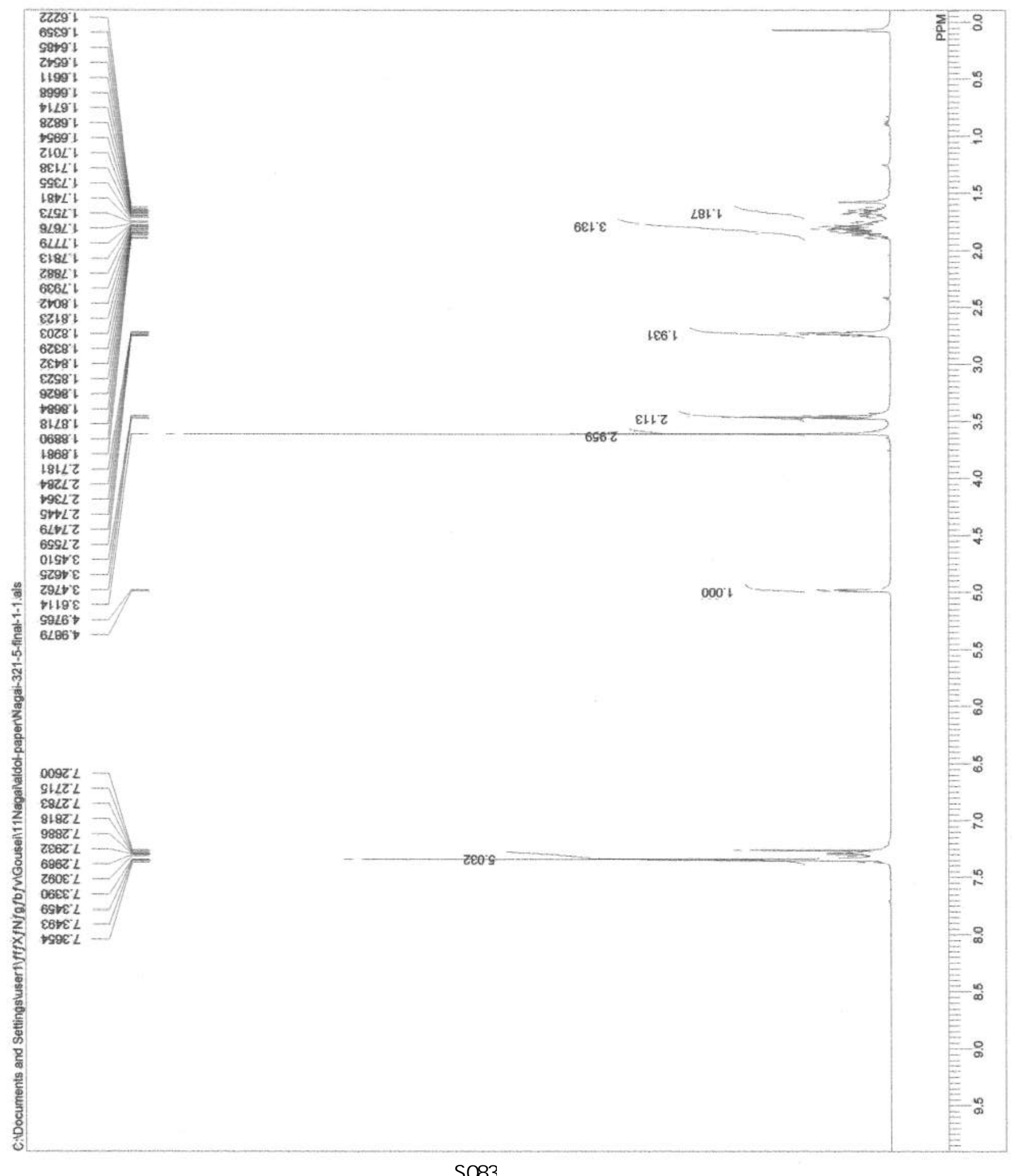



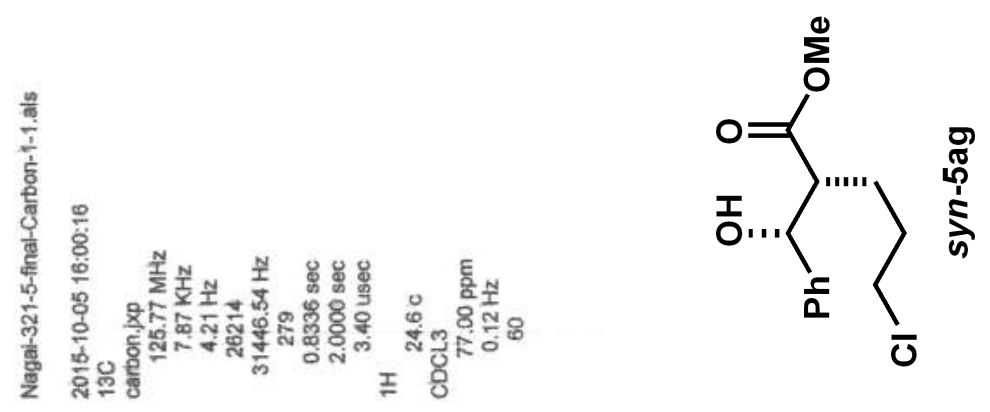

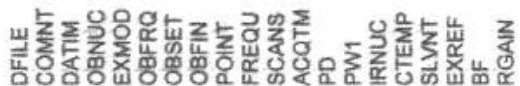

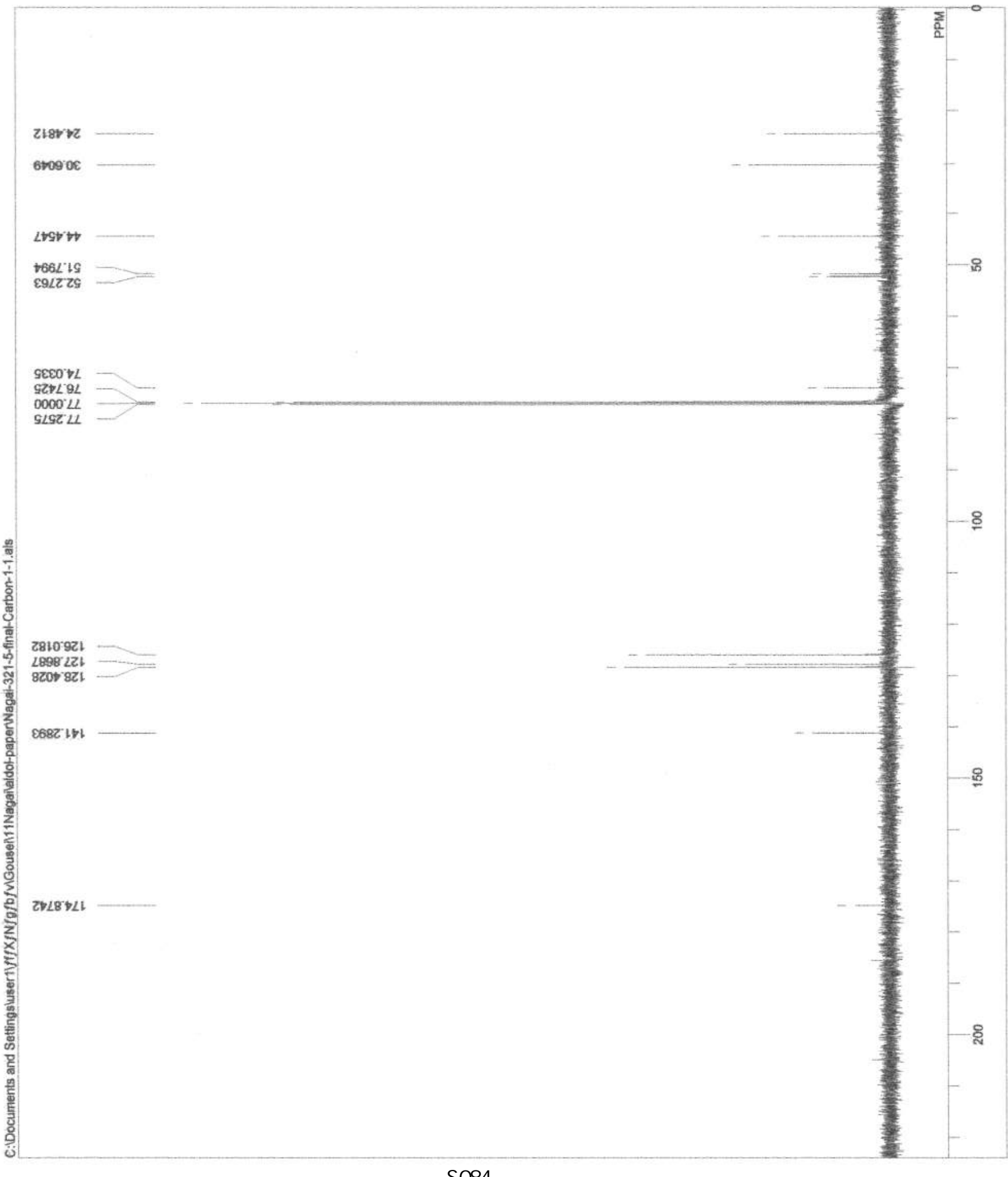



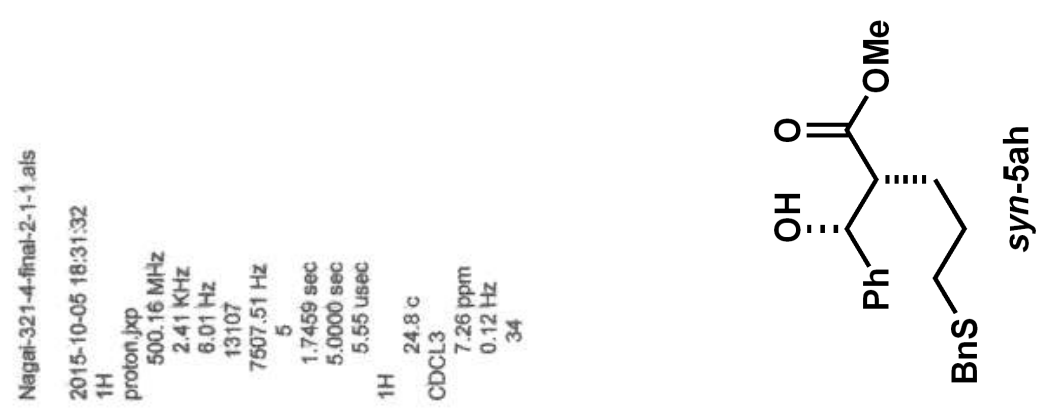

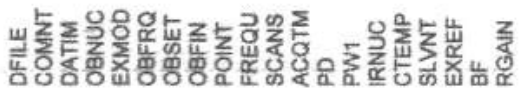

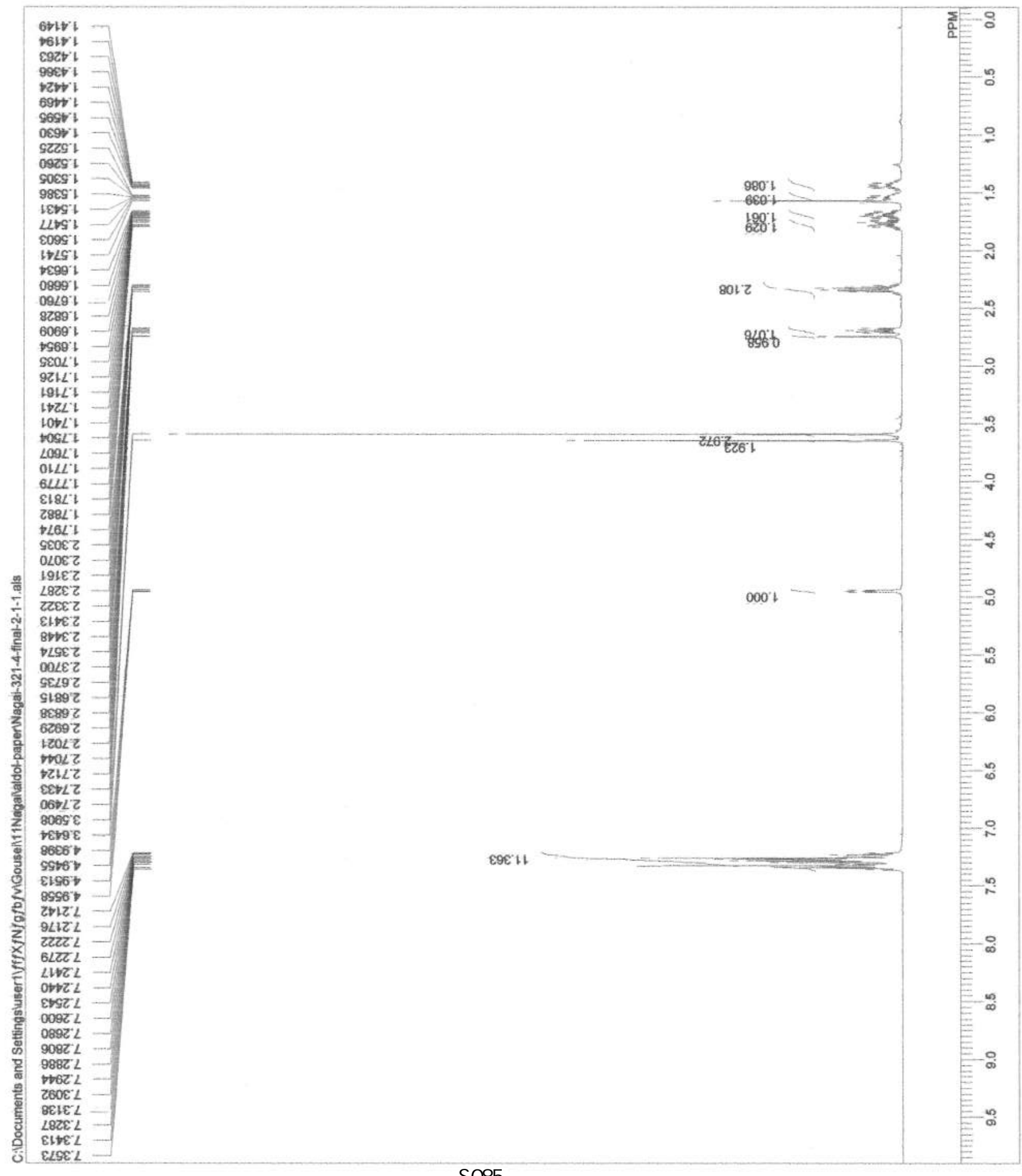



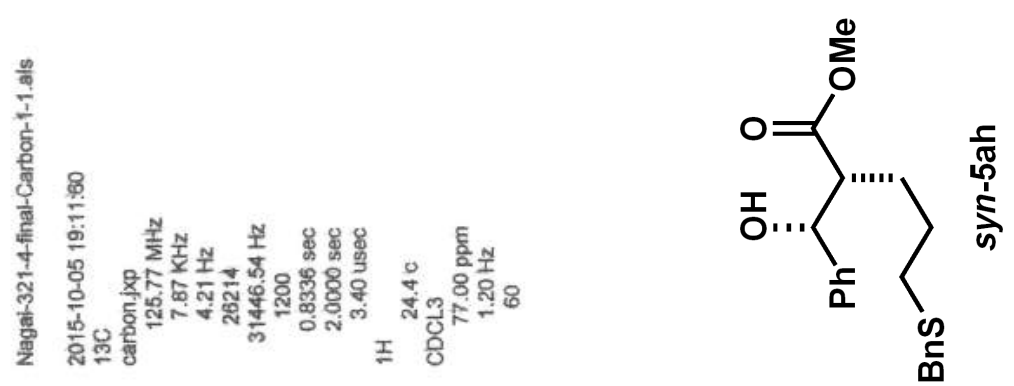

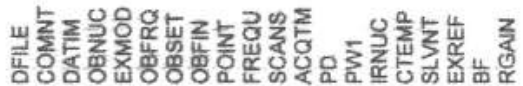

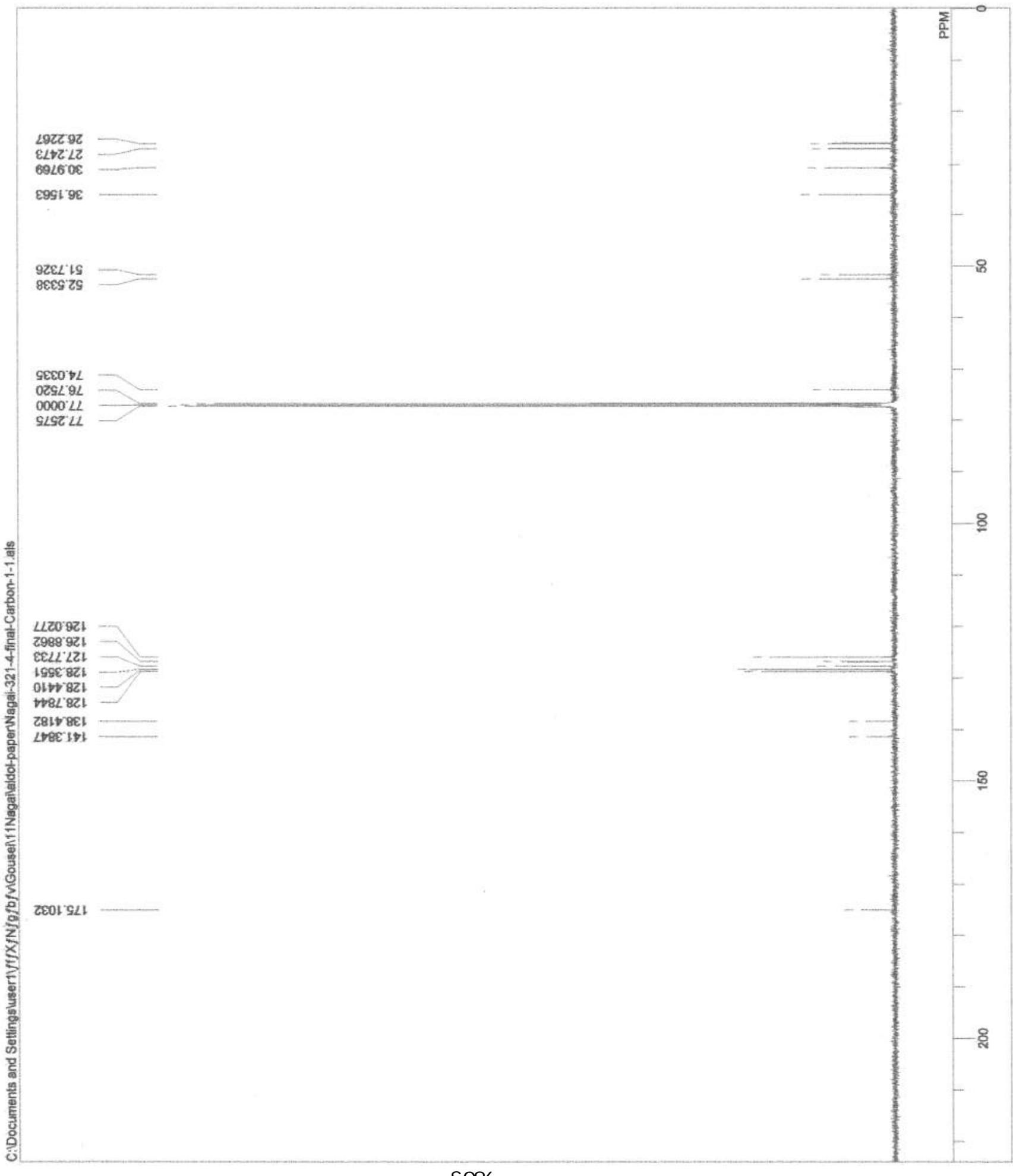




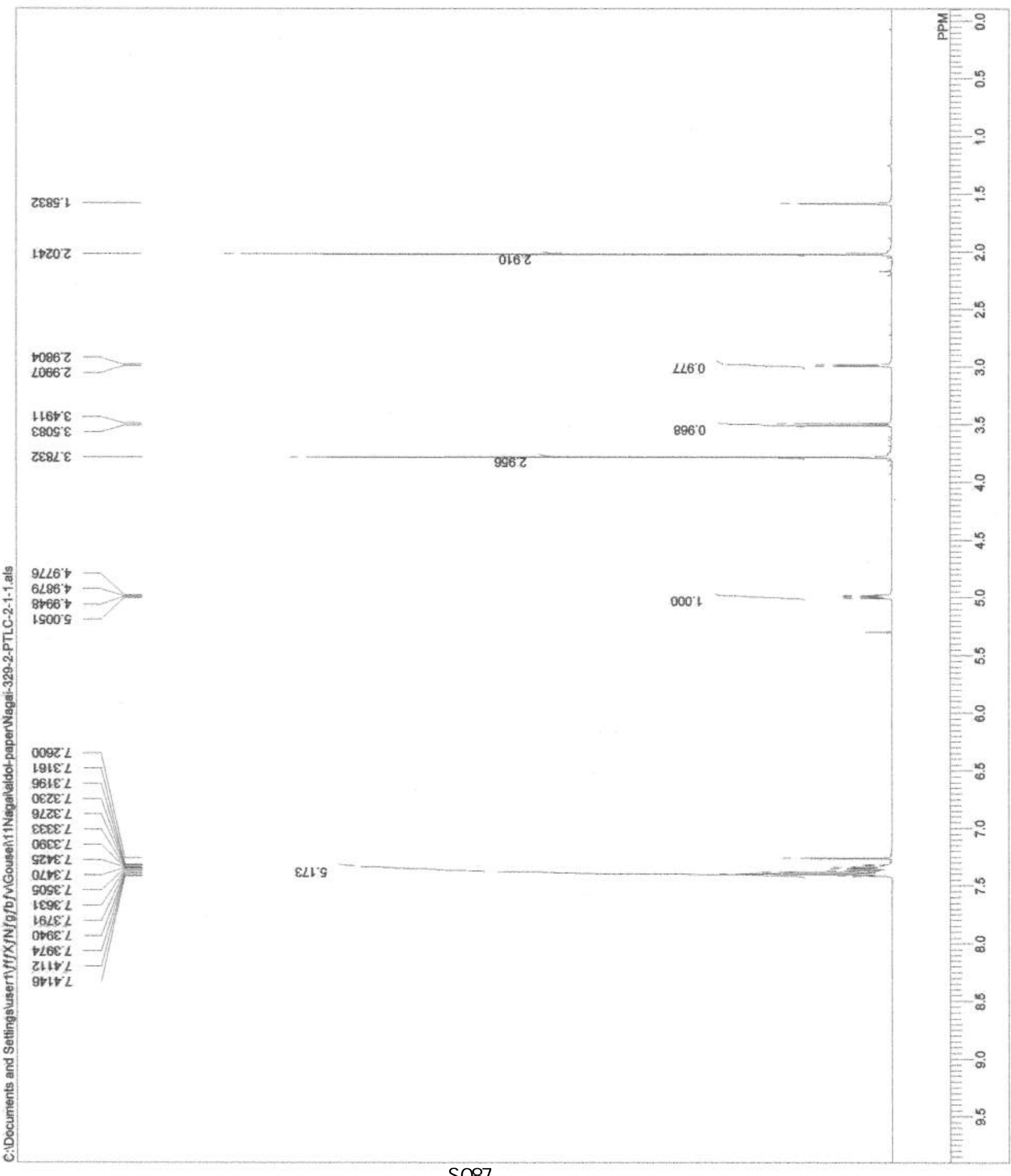




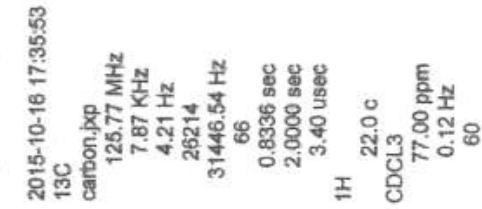

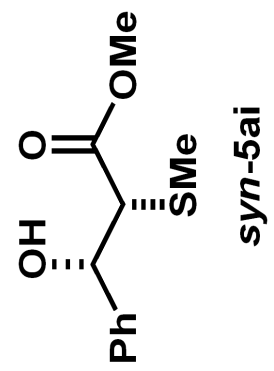

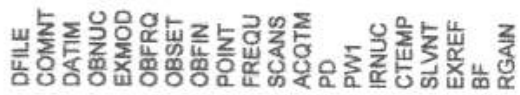

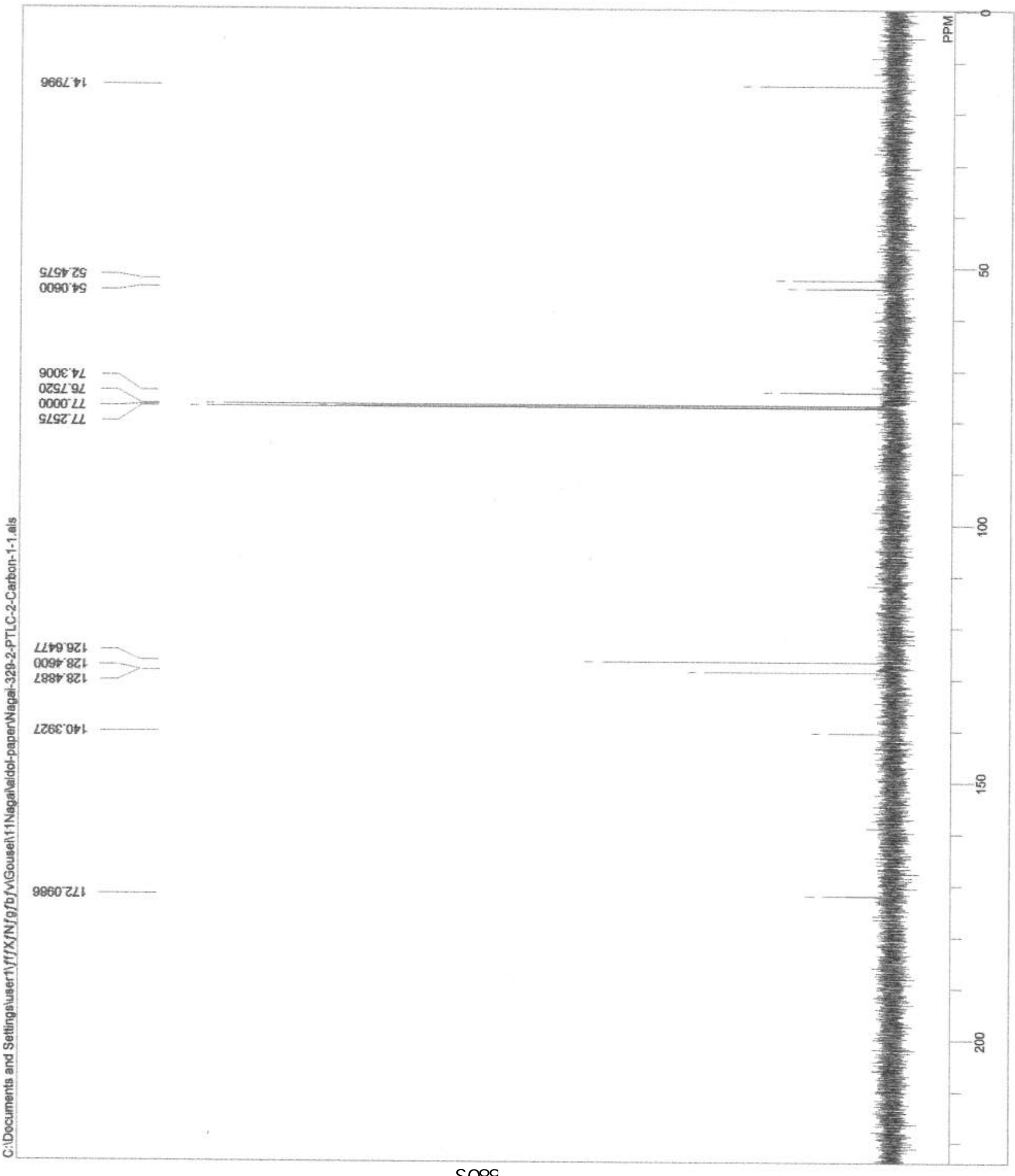



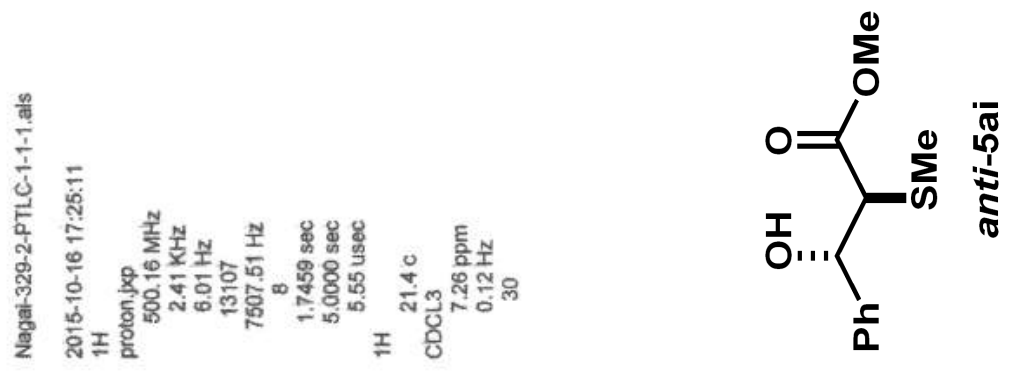

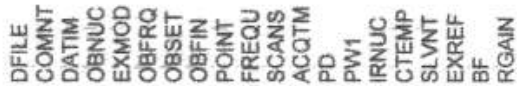

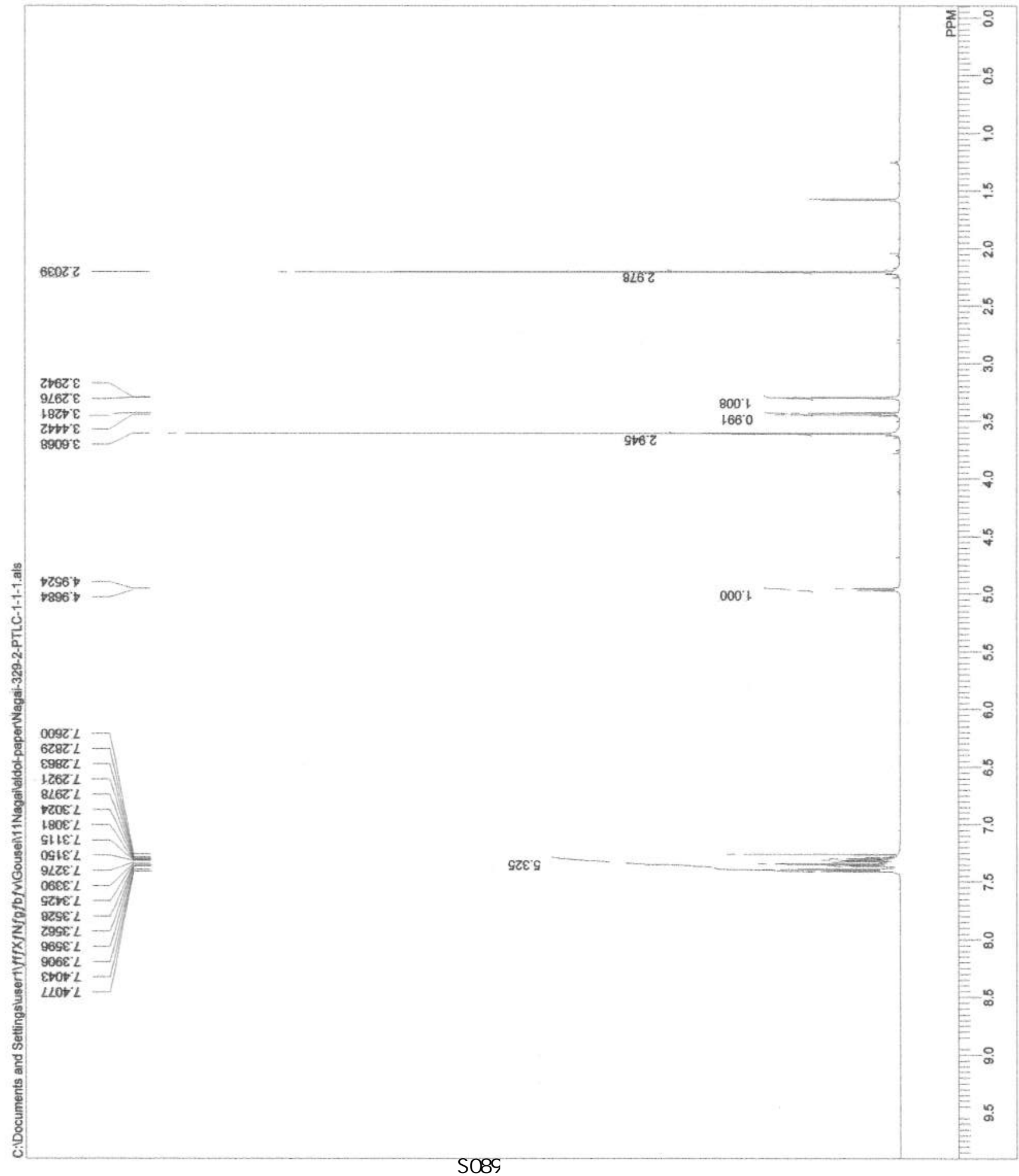



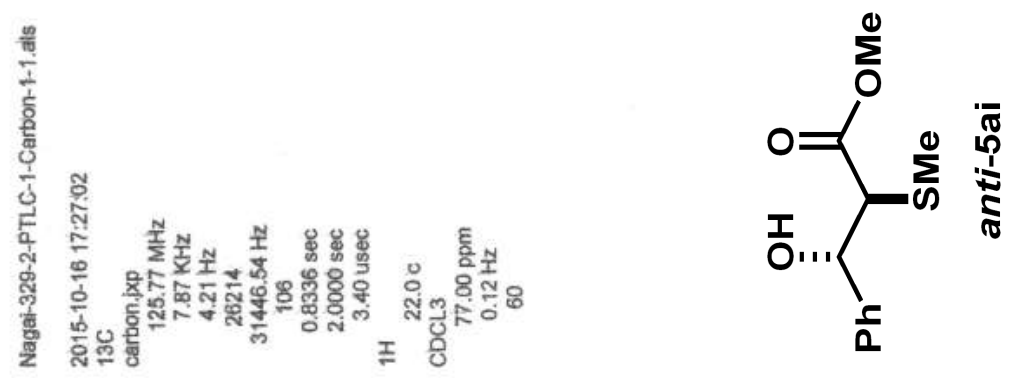

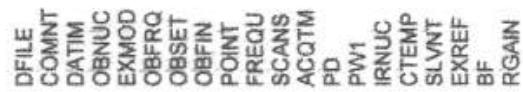

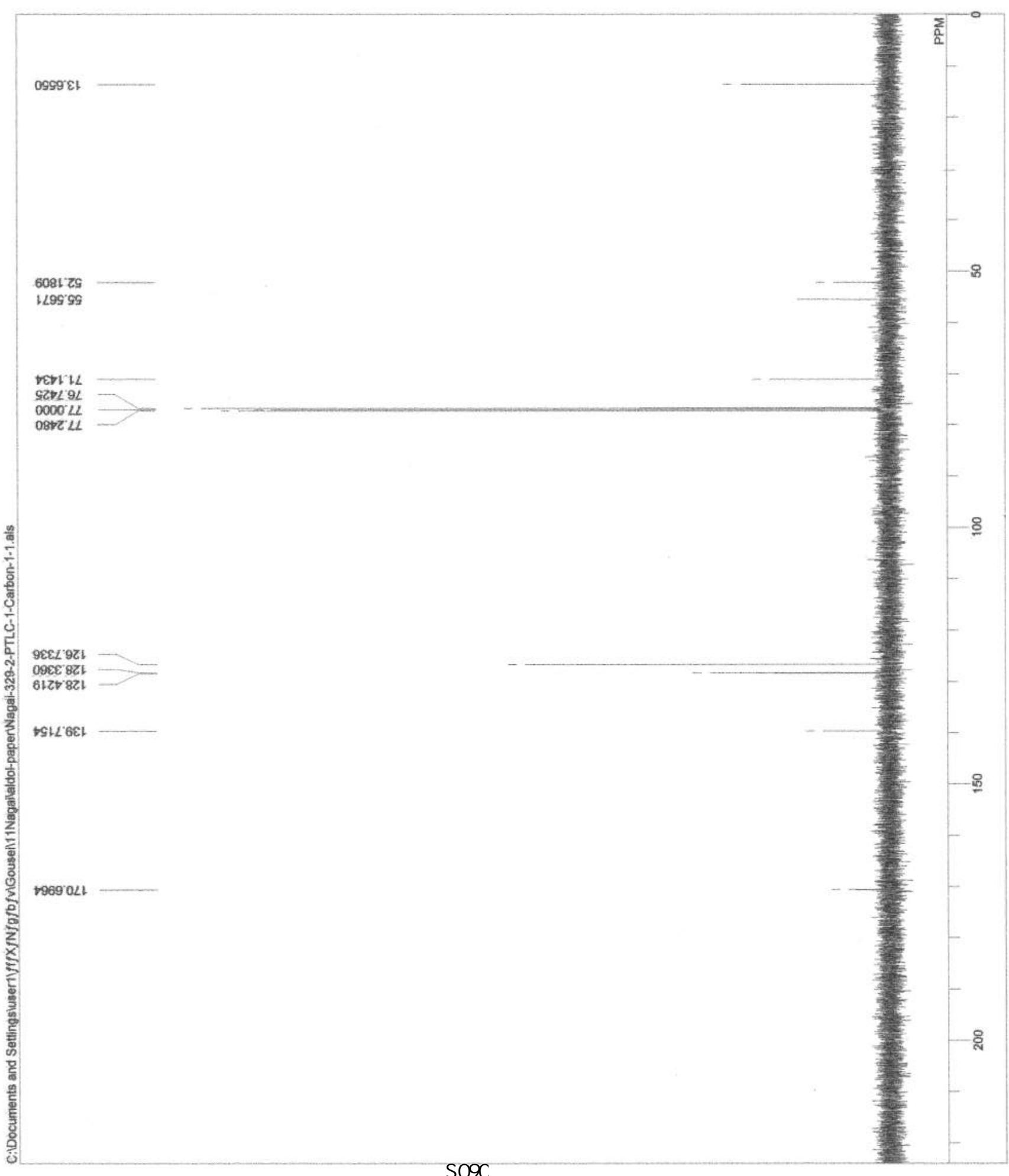



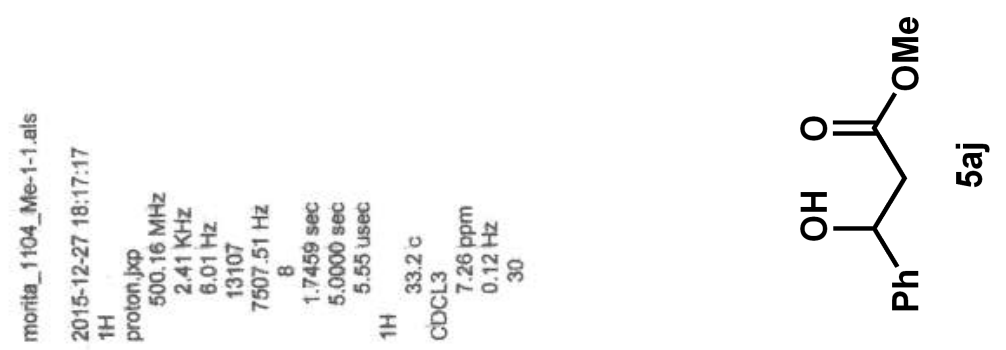

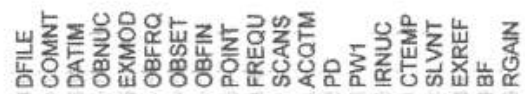

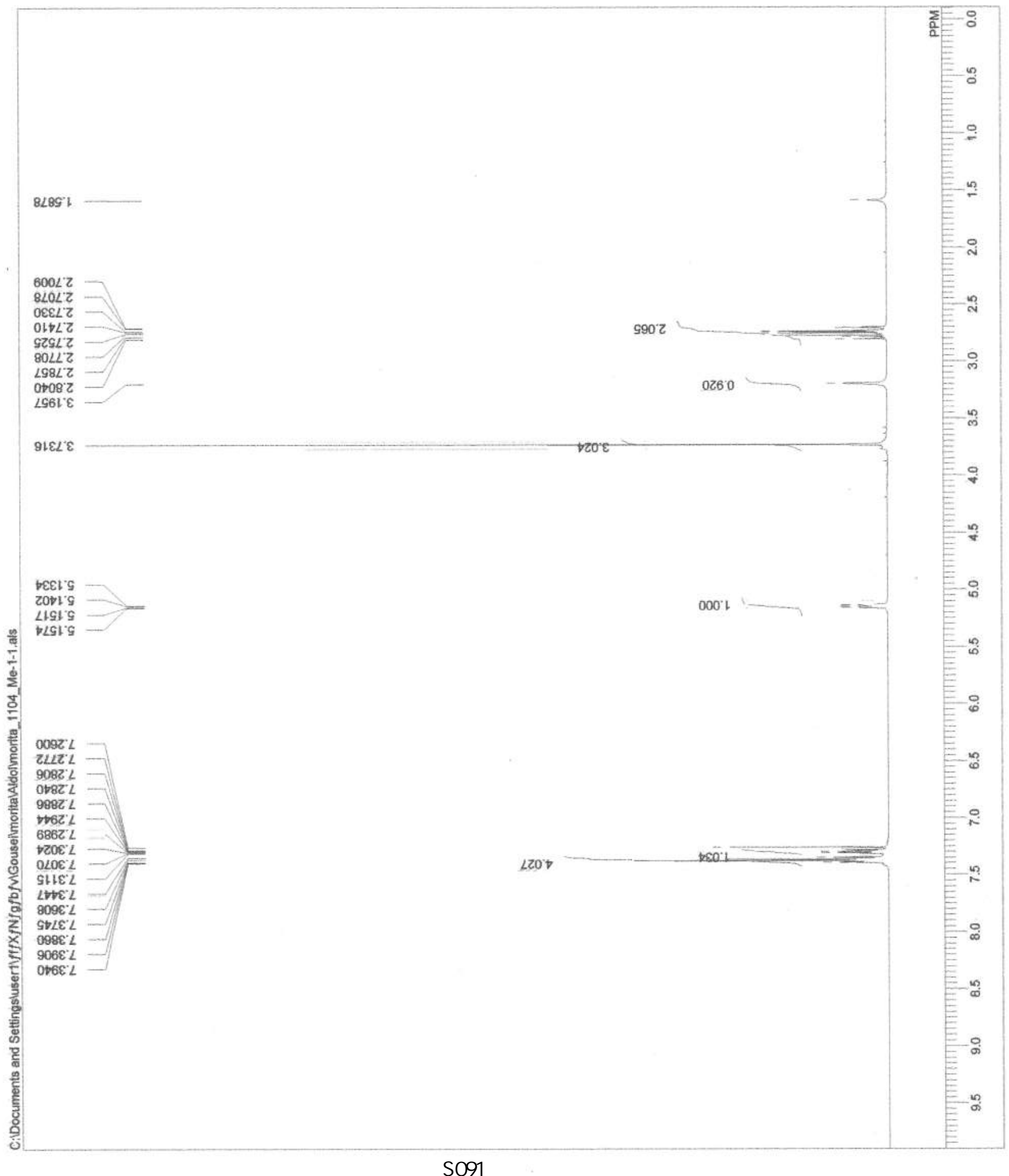




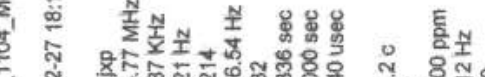

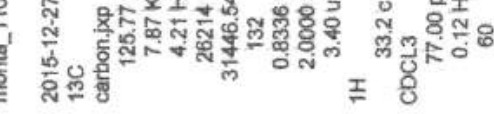

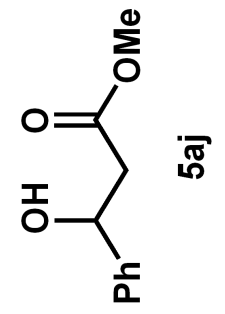

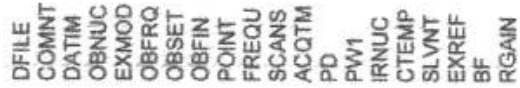

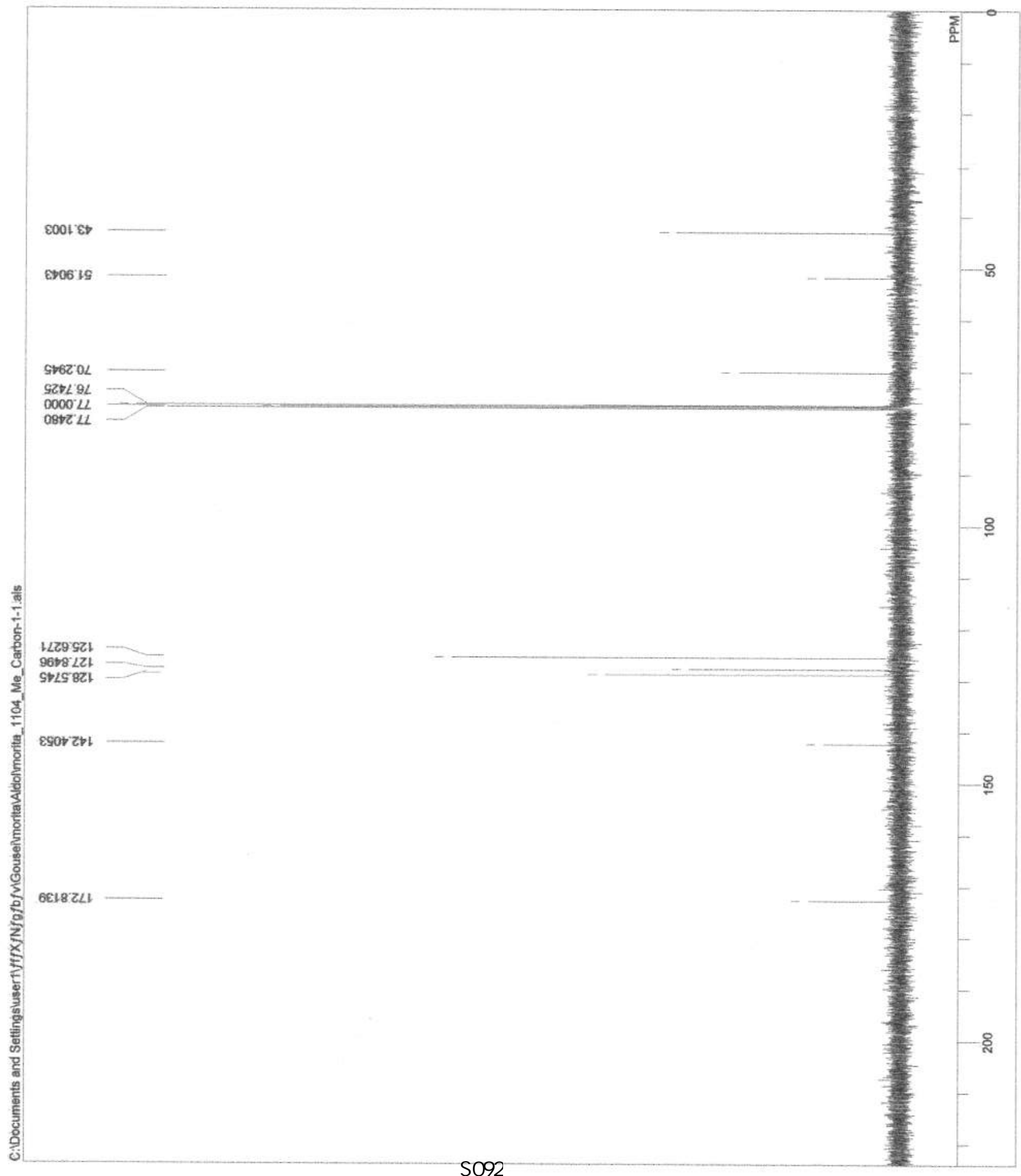




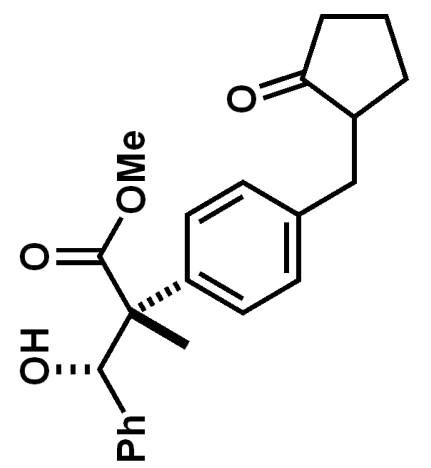

㐫

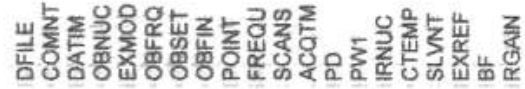

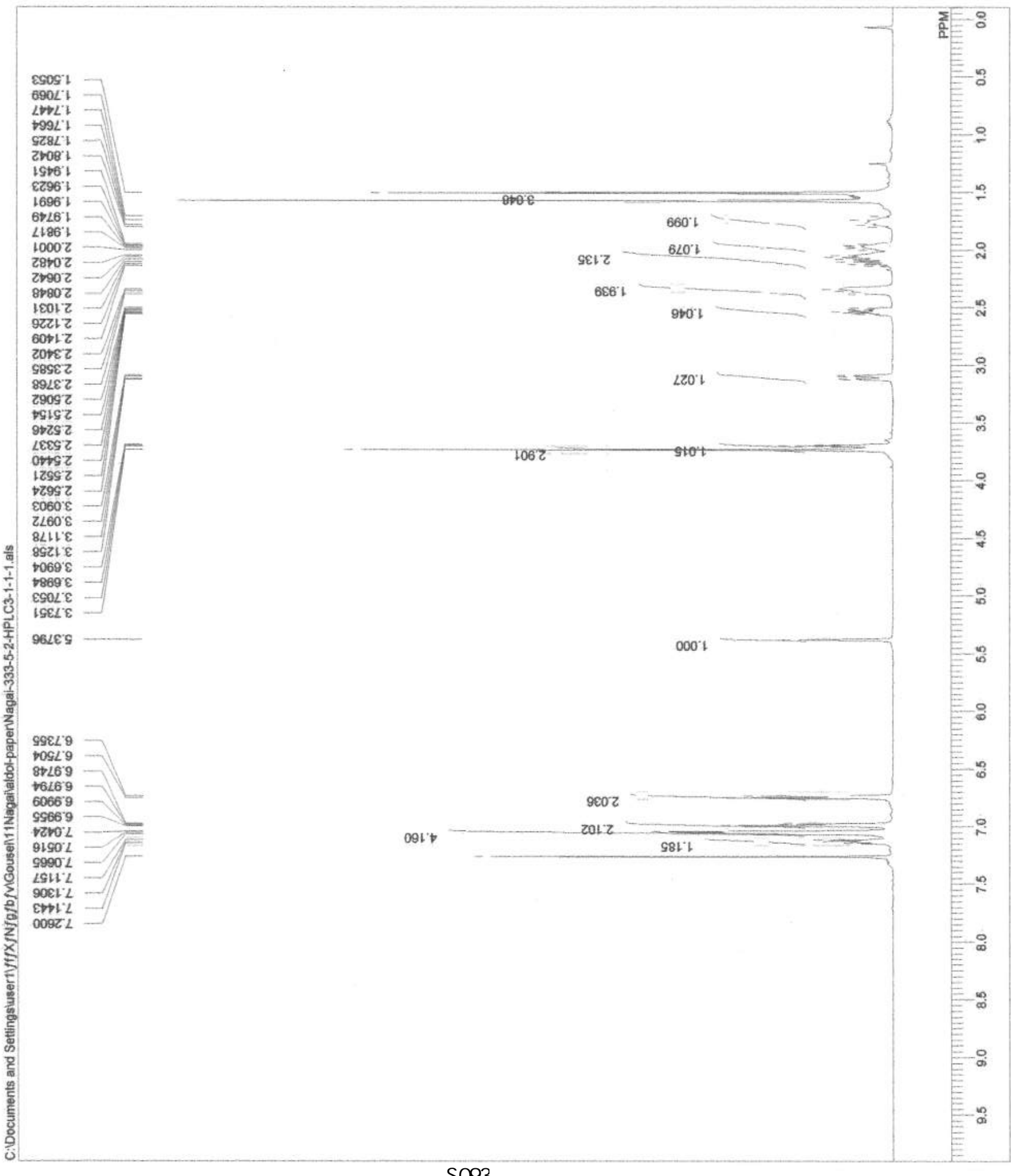




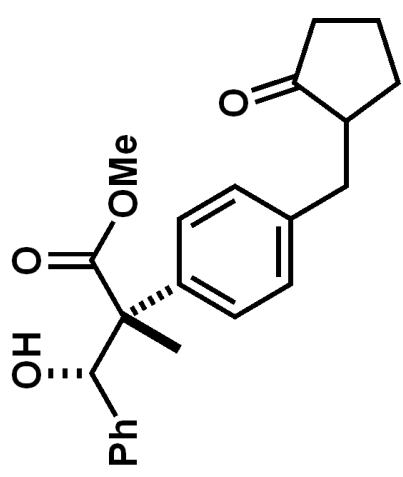

$\frac{y}{\sigma}$
$\frac{1}{5}$
$\frac{5}{5}$

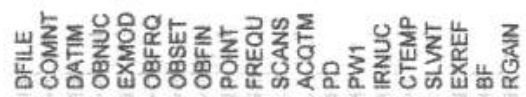

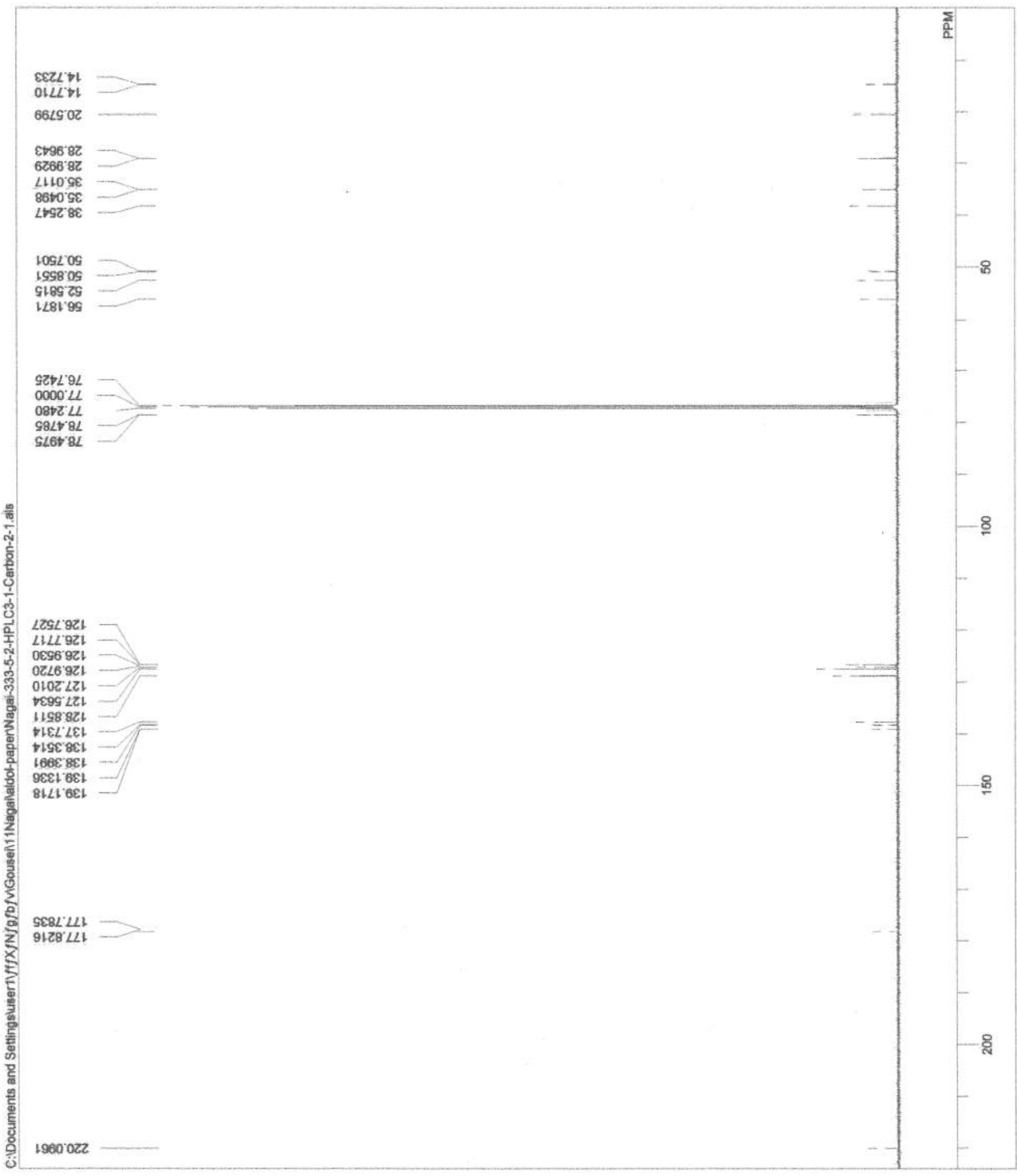



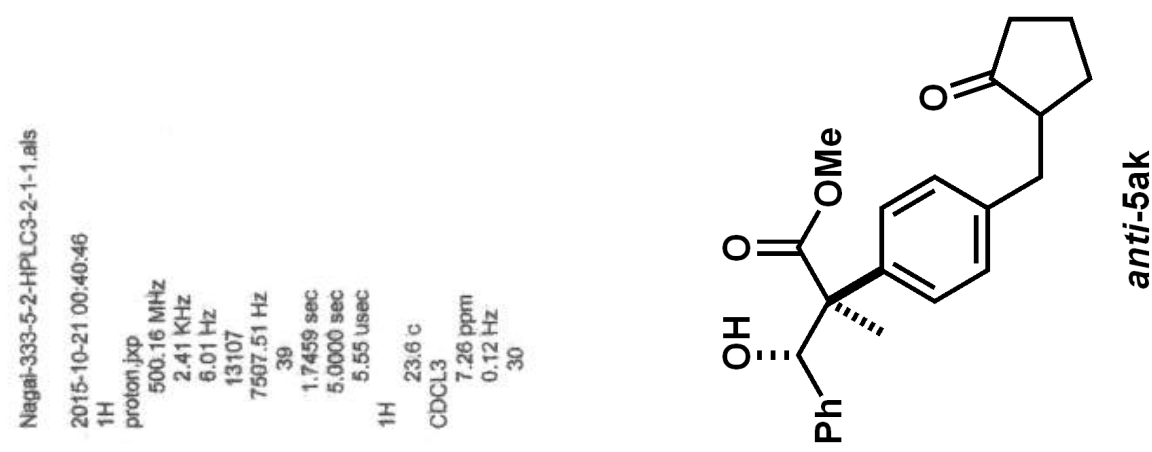

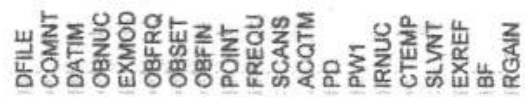

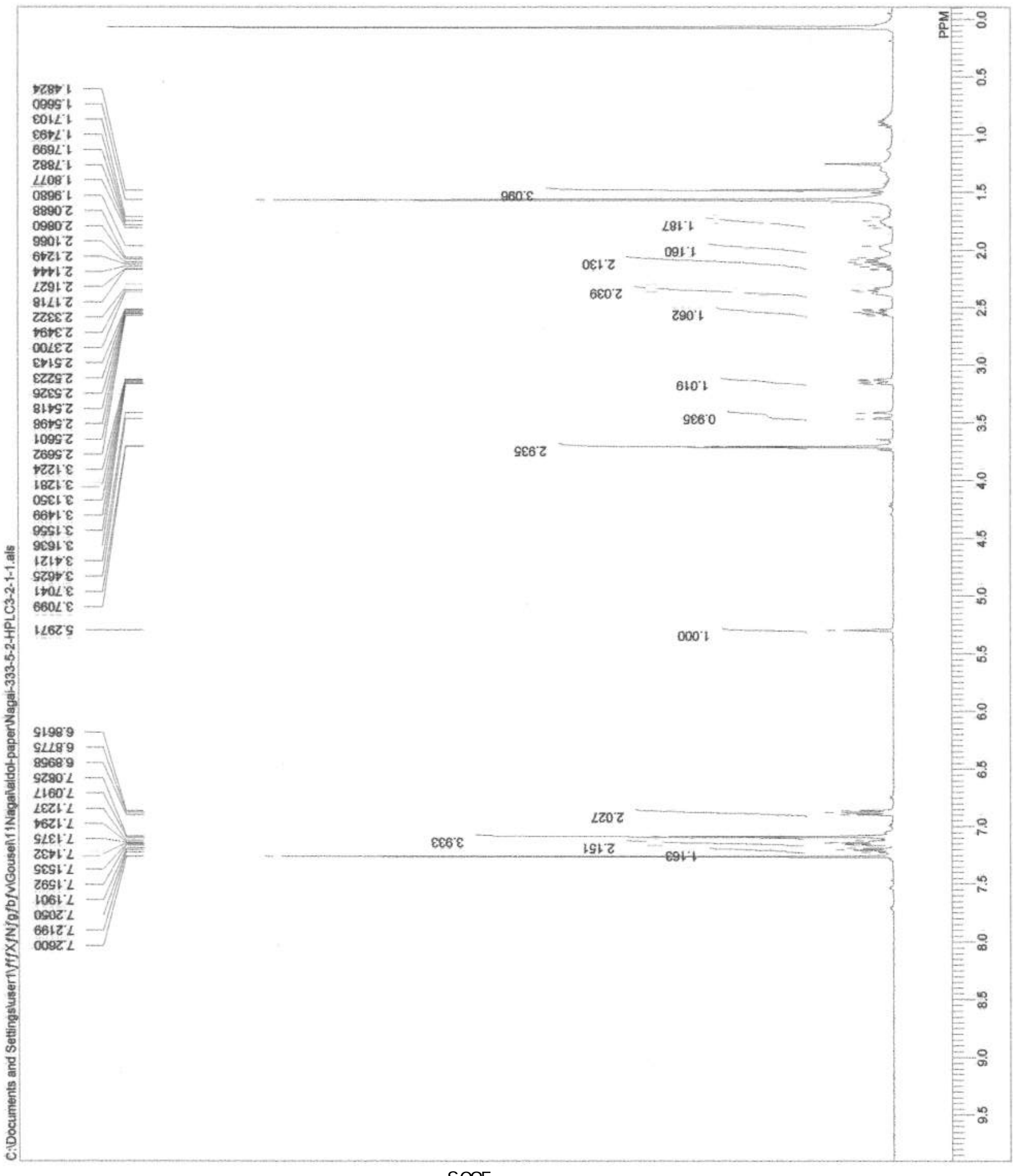



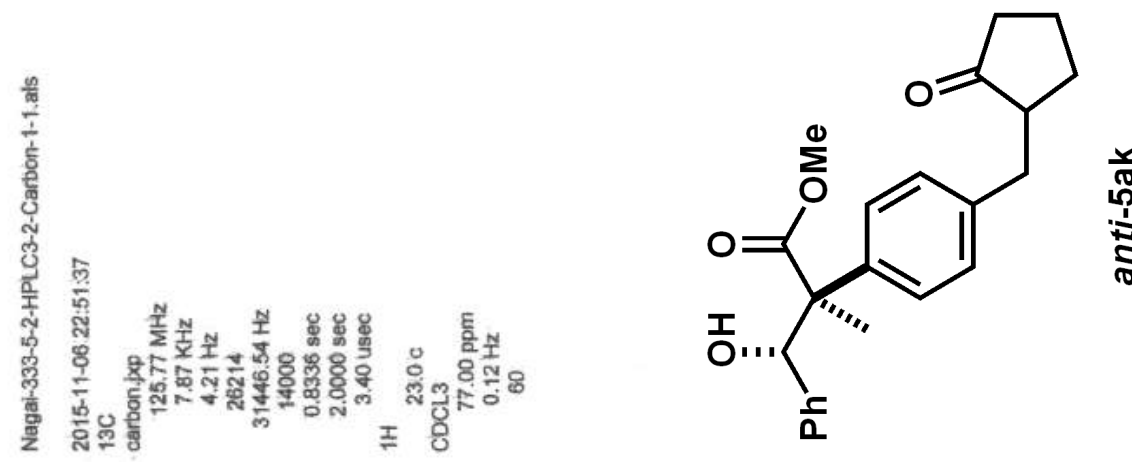

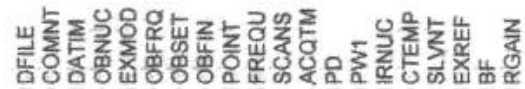

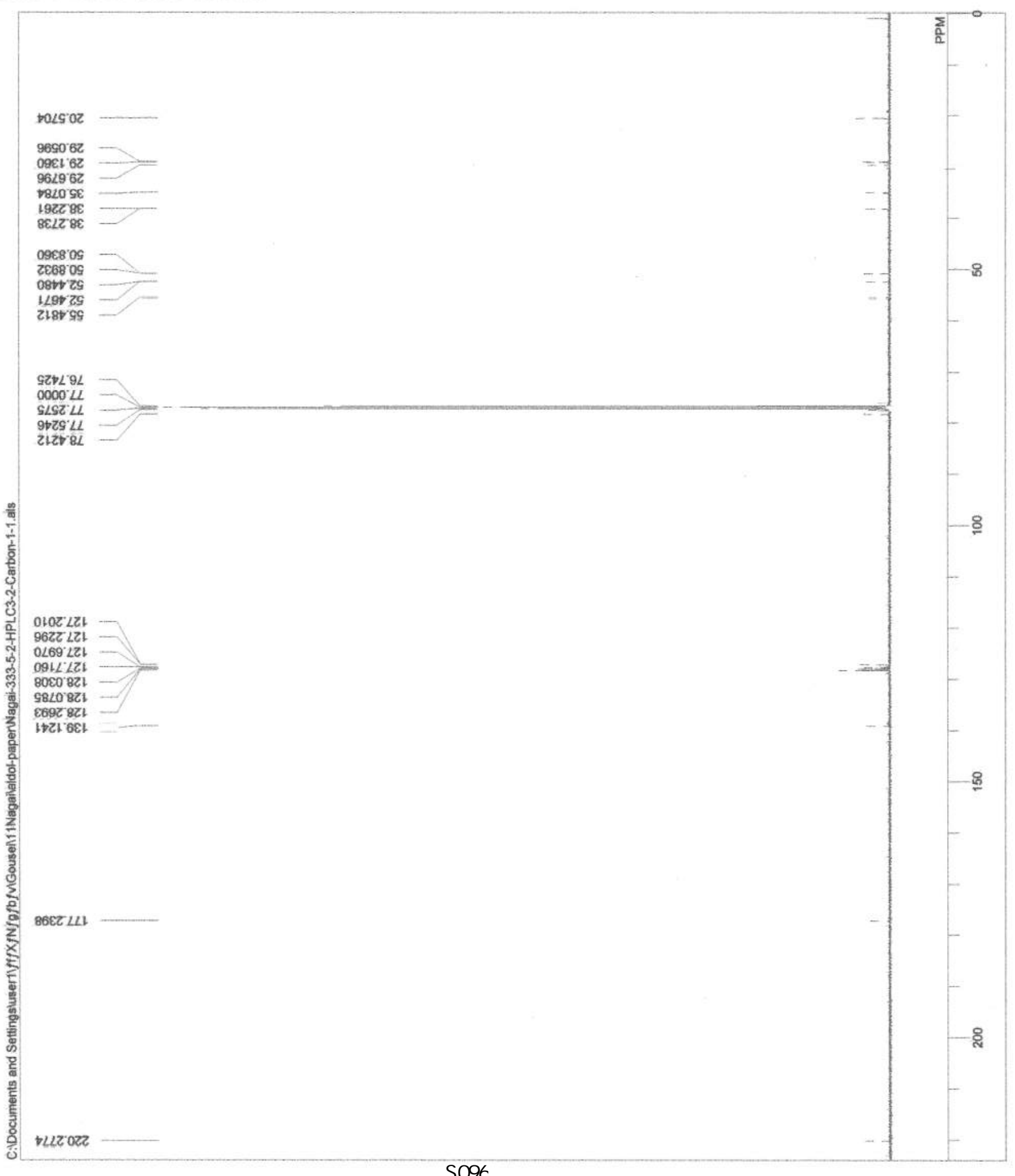




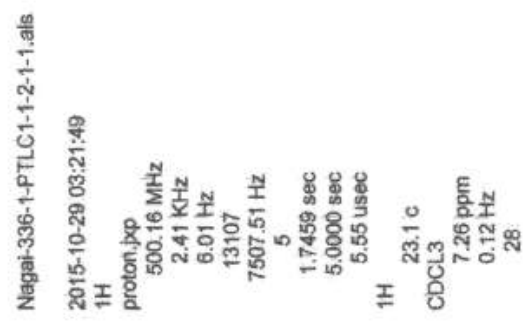

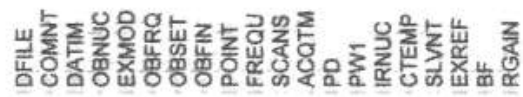
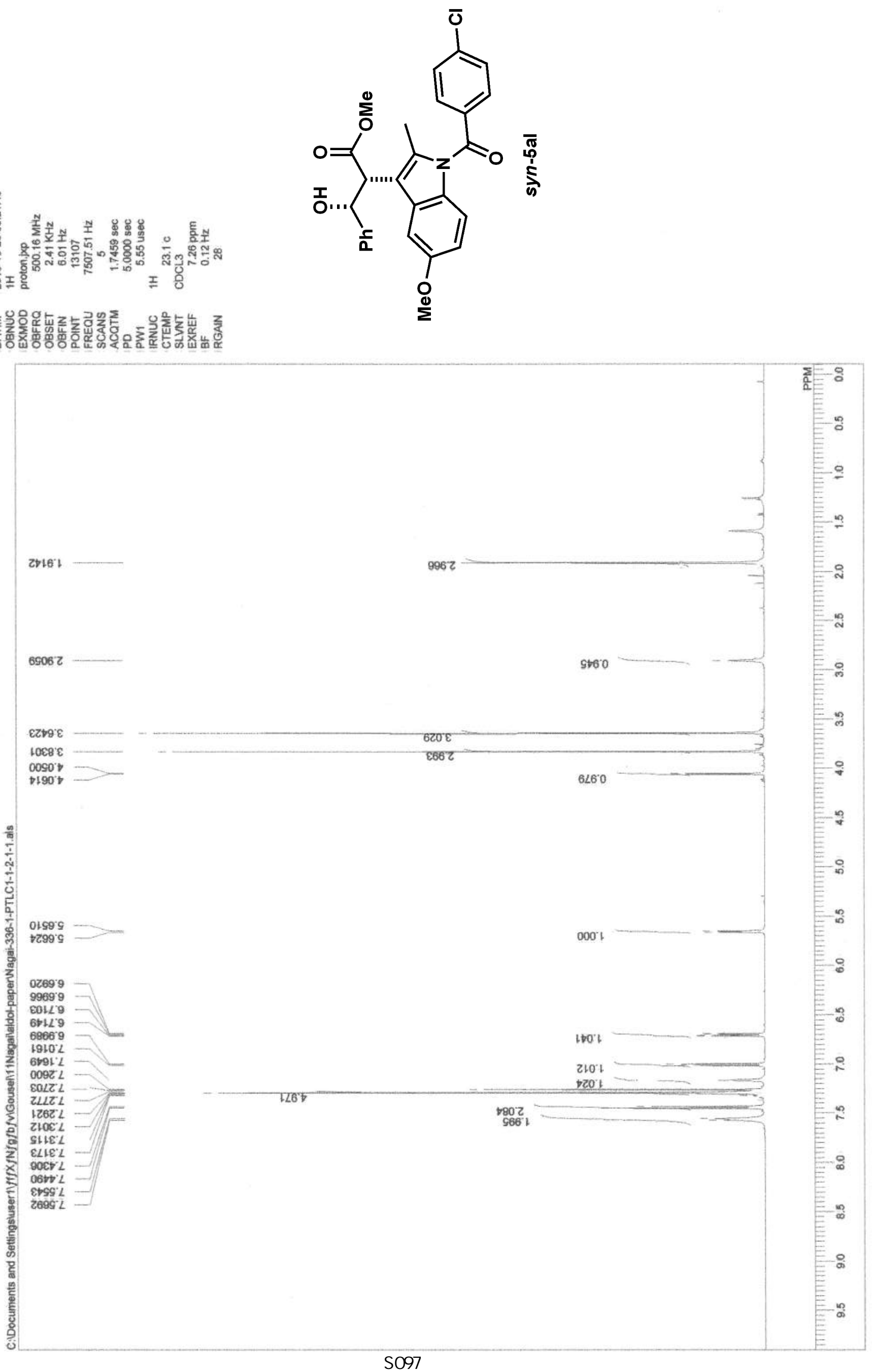
<smiles></smiles>

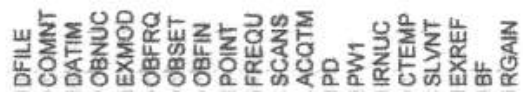

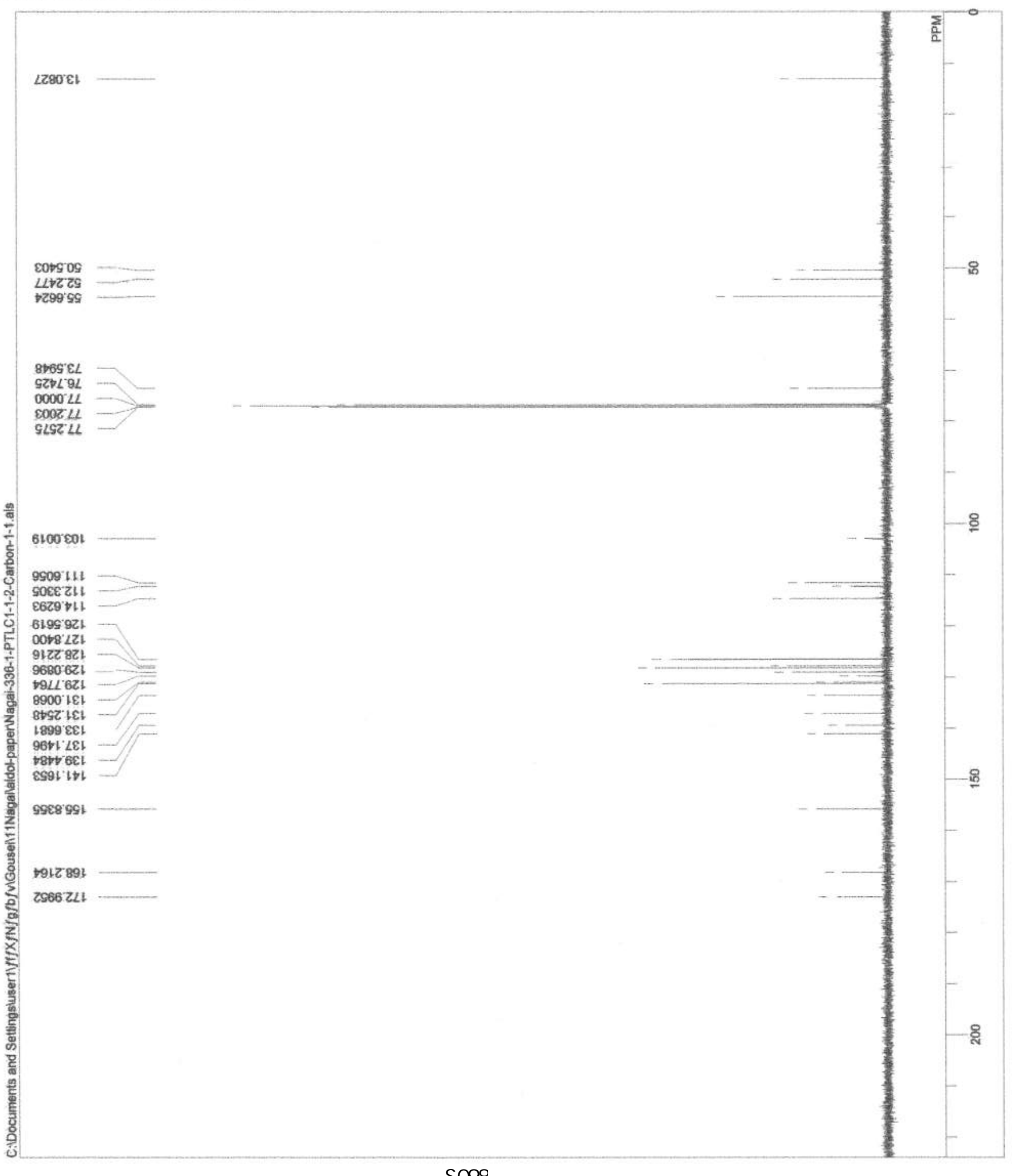


<smiles></smiles>

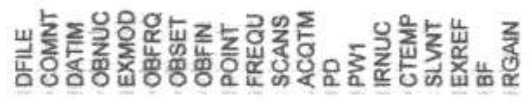

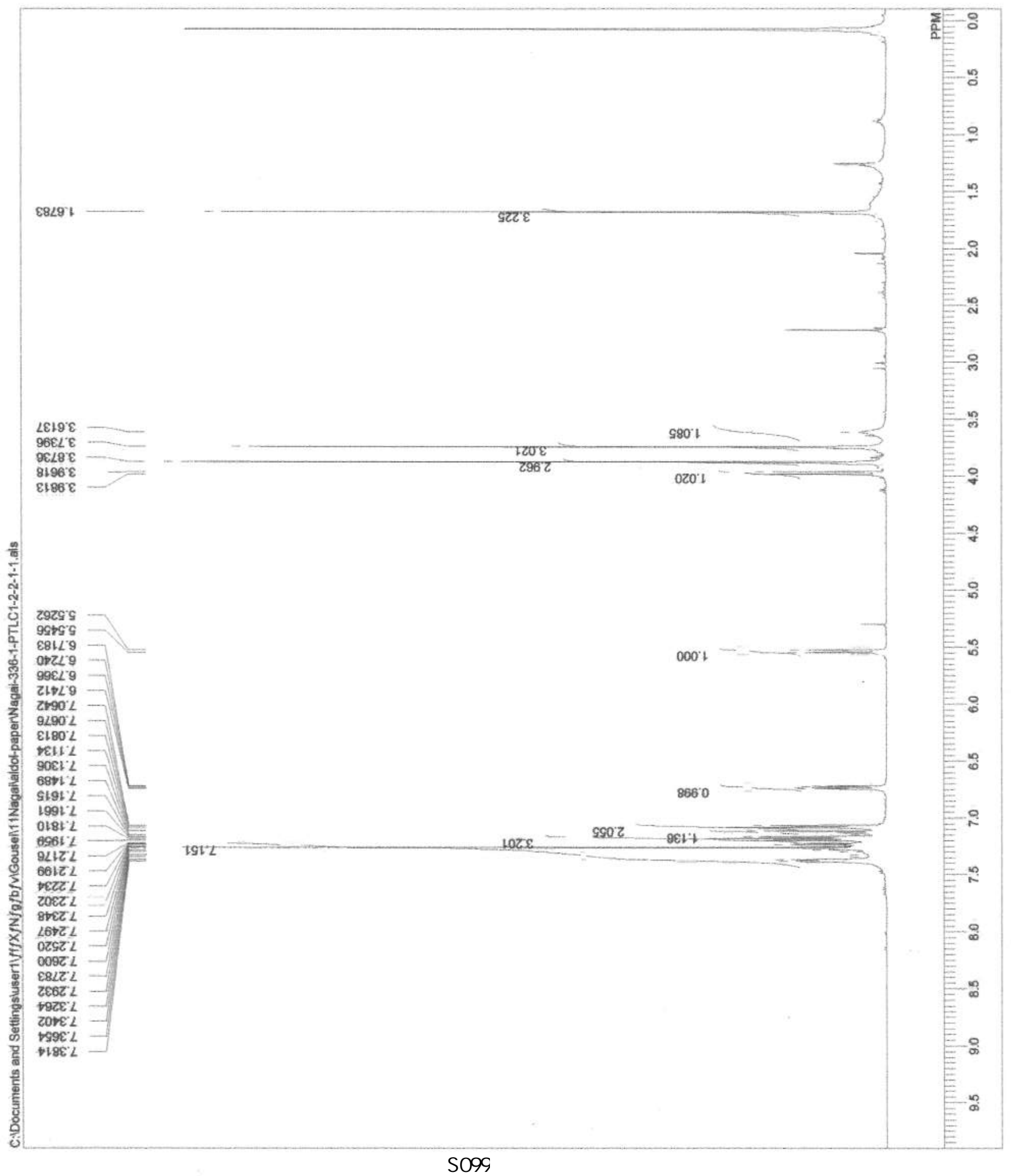




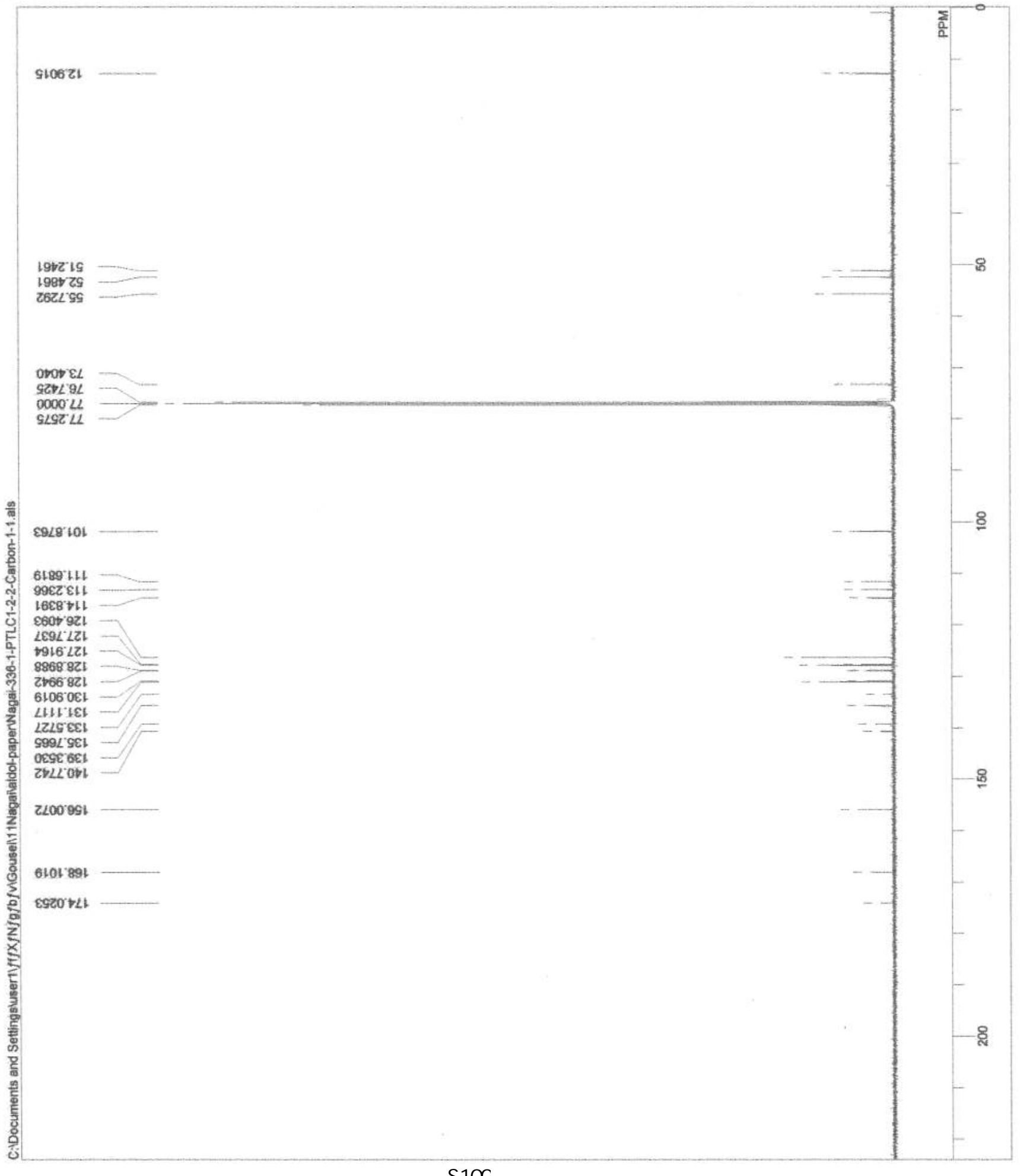




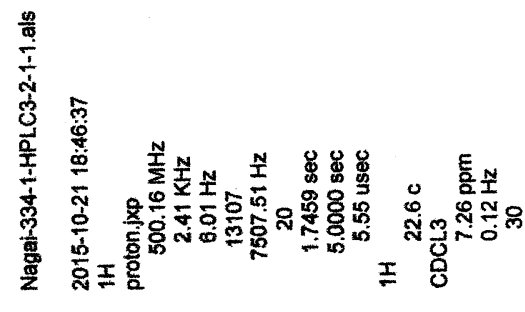

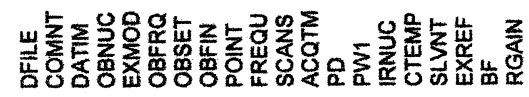
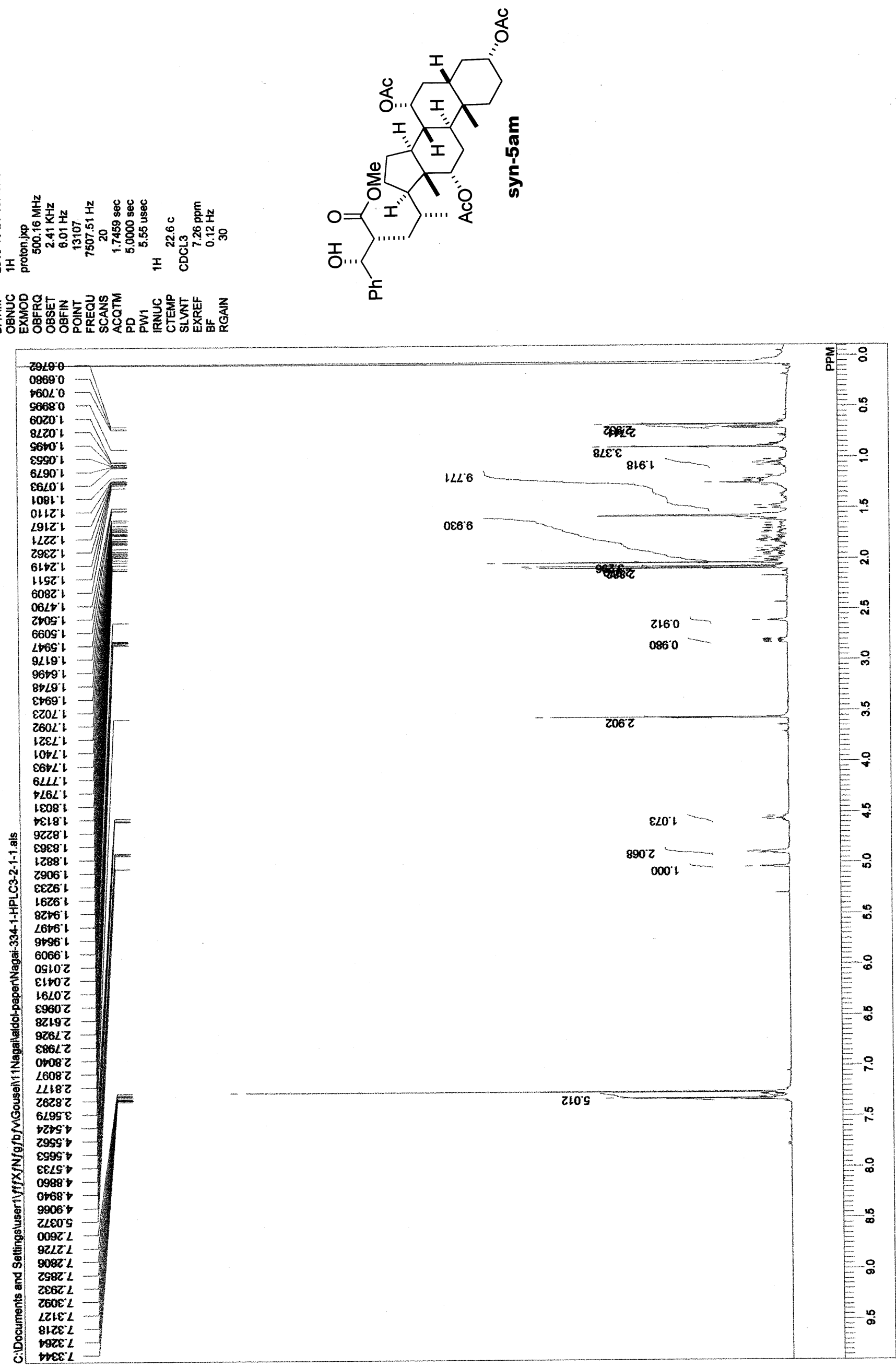


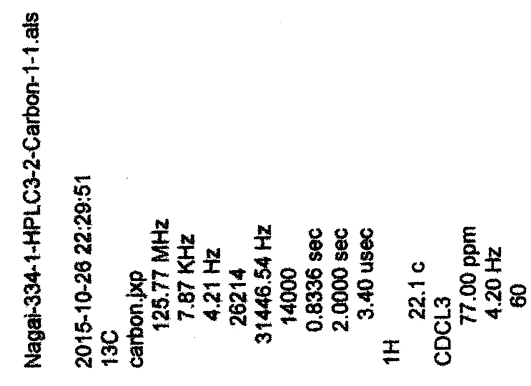

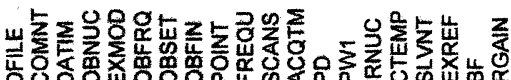

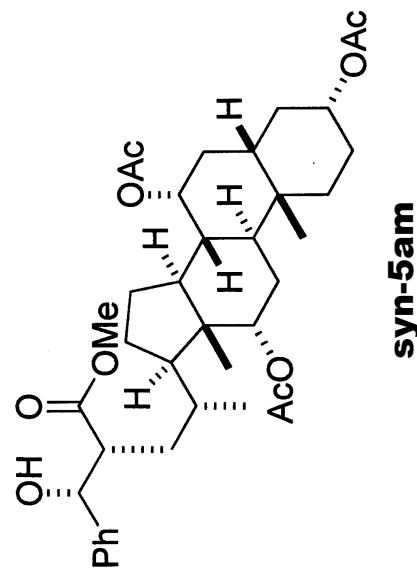

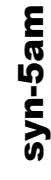

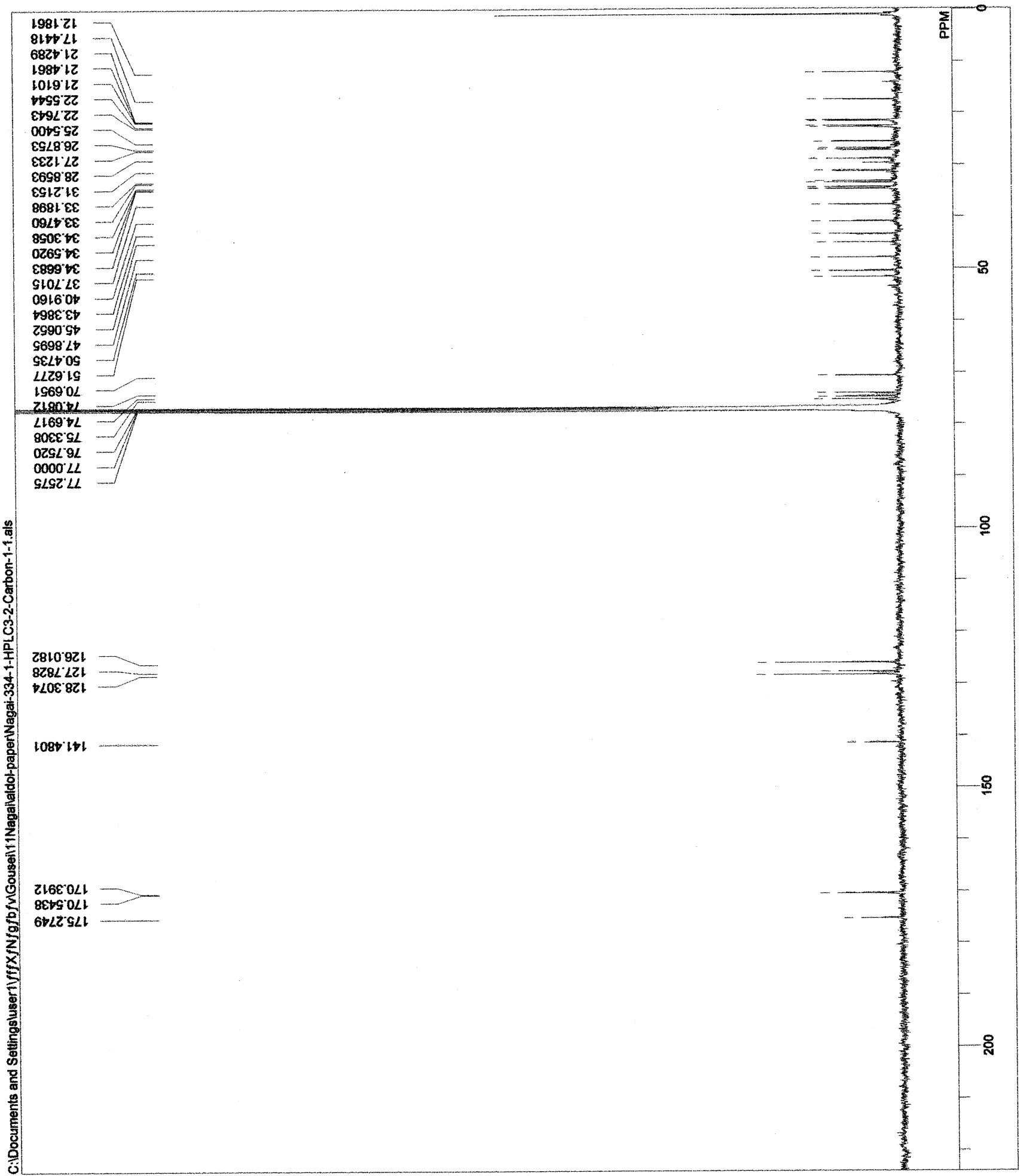



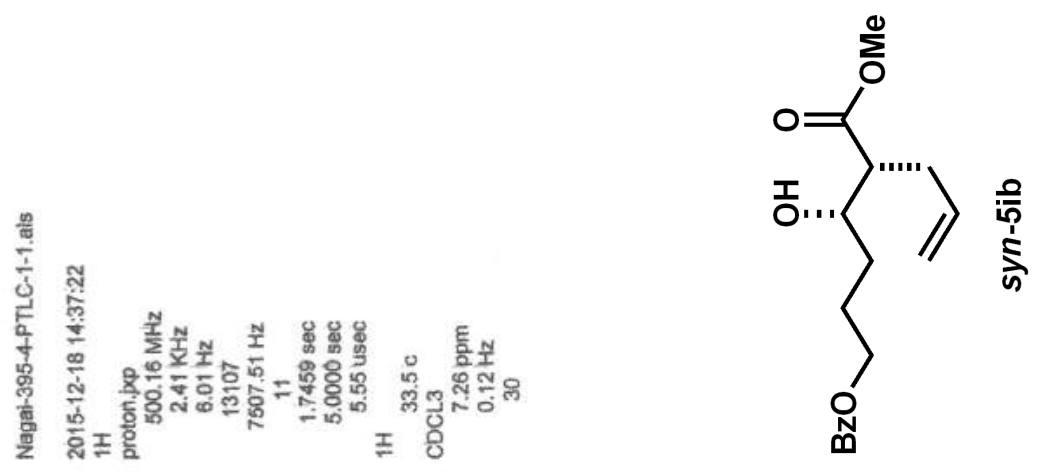

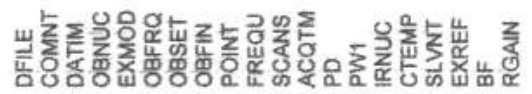

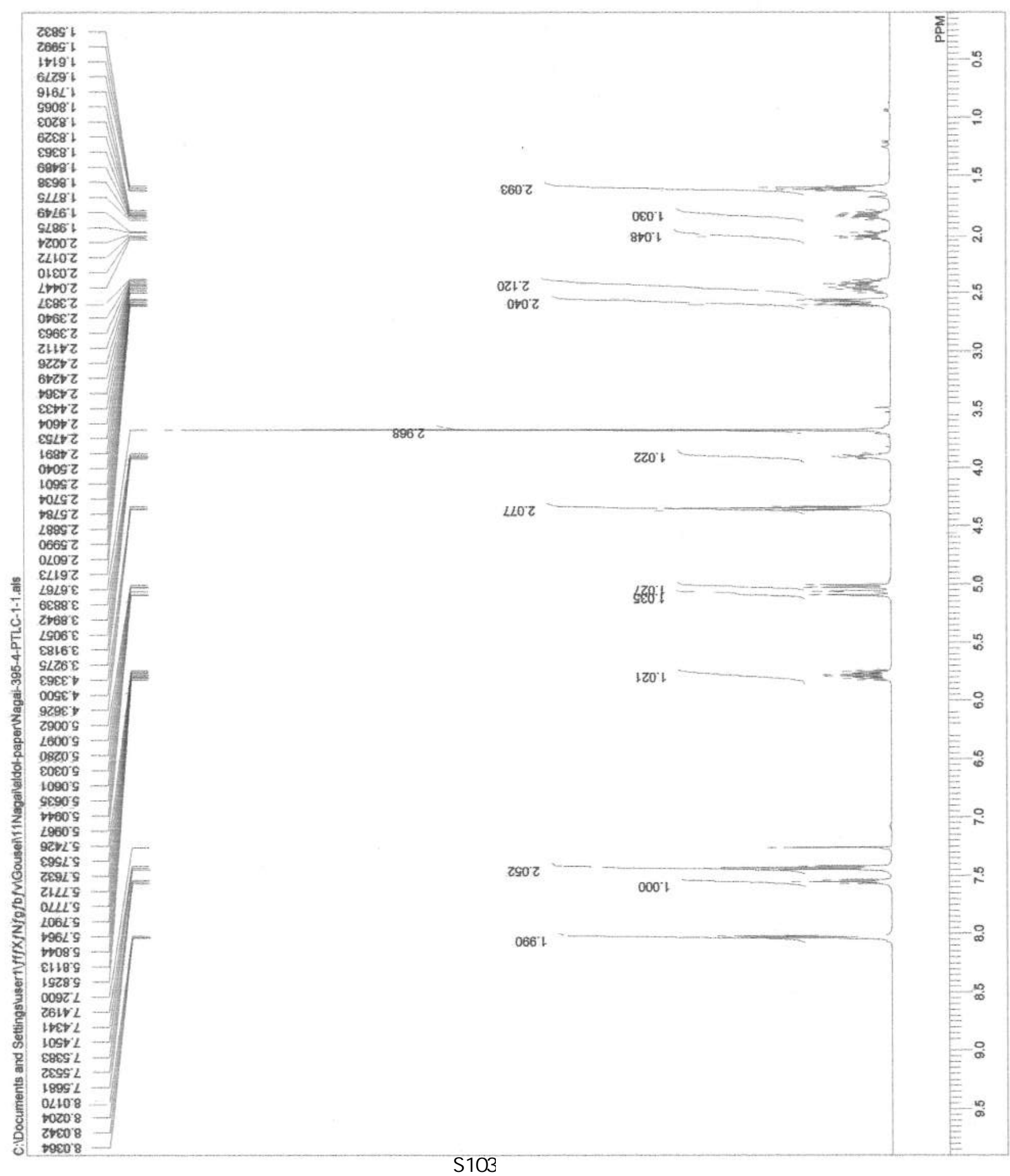



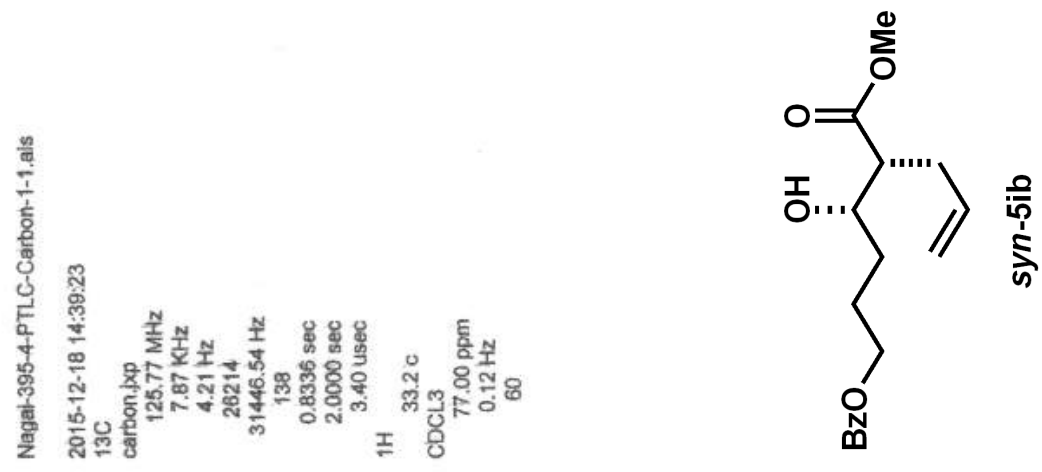

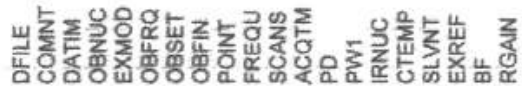

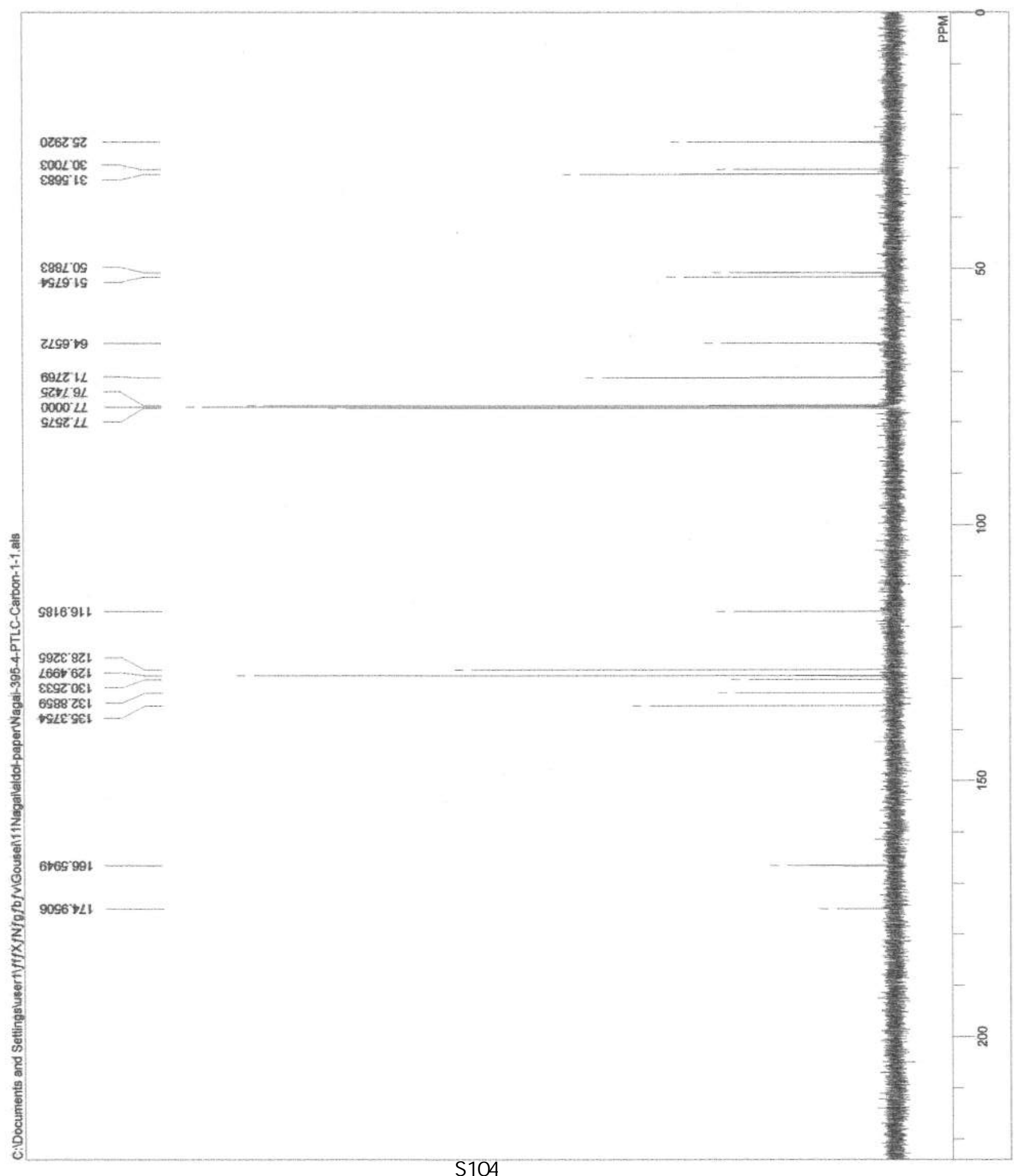



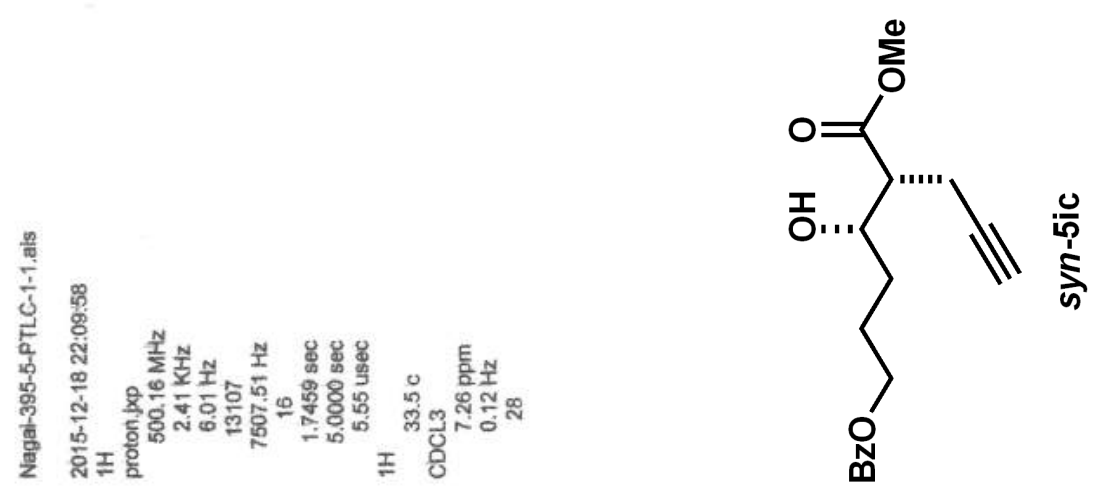

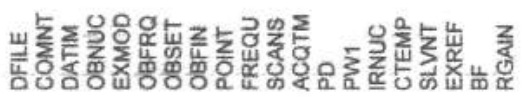

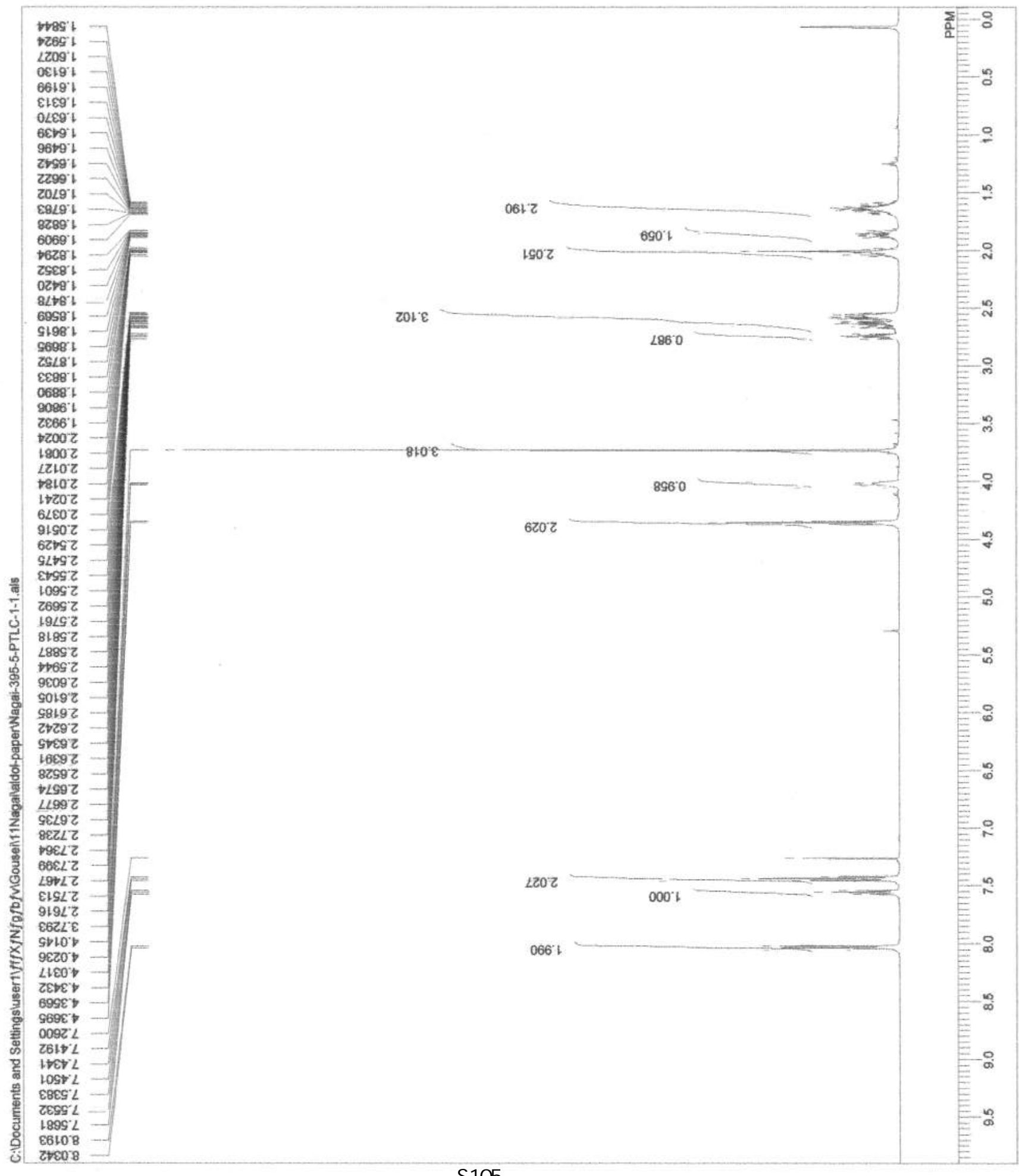



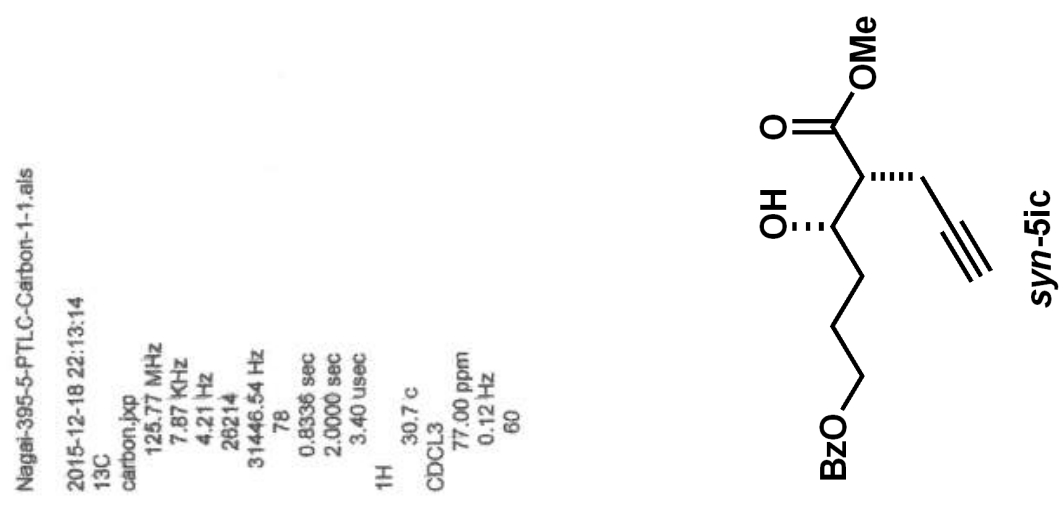

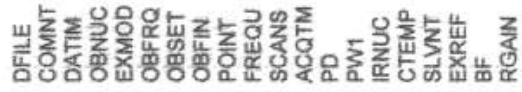

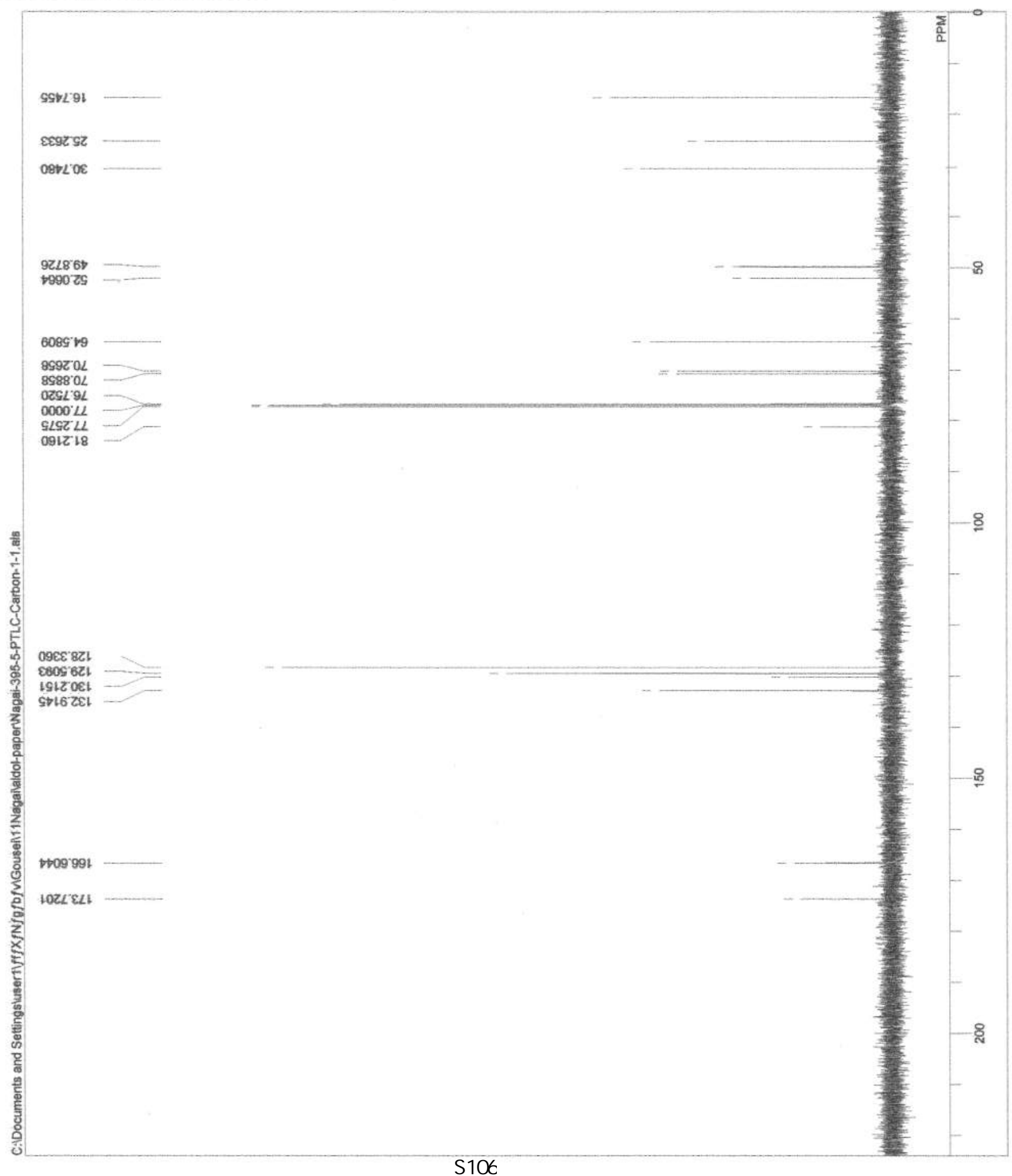




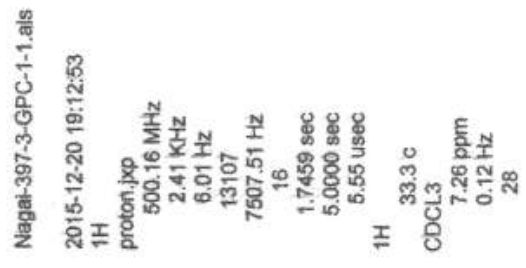

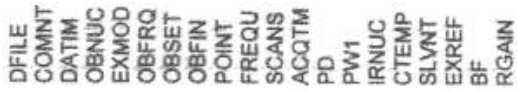
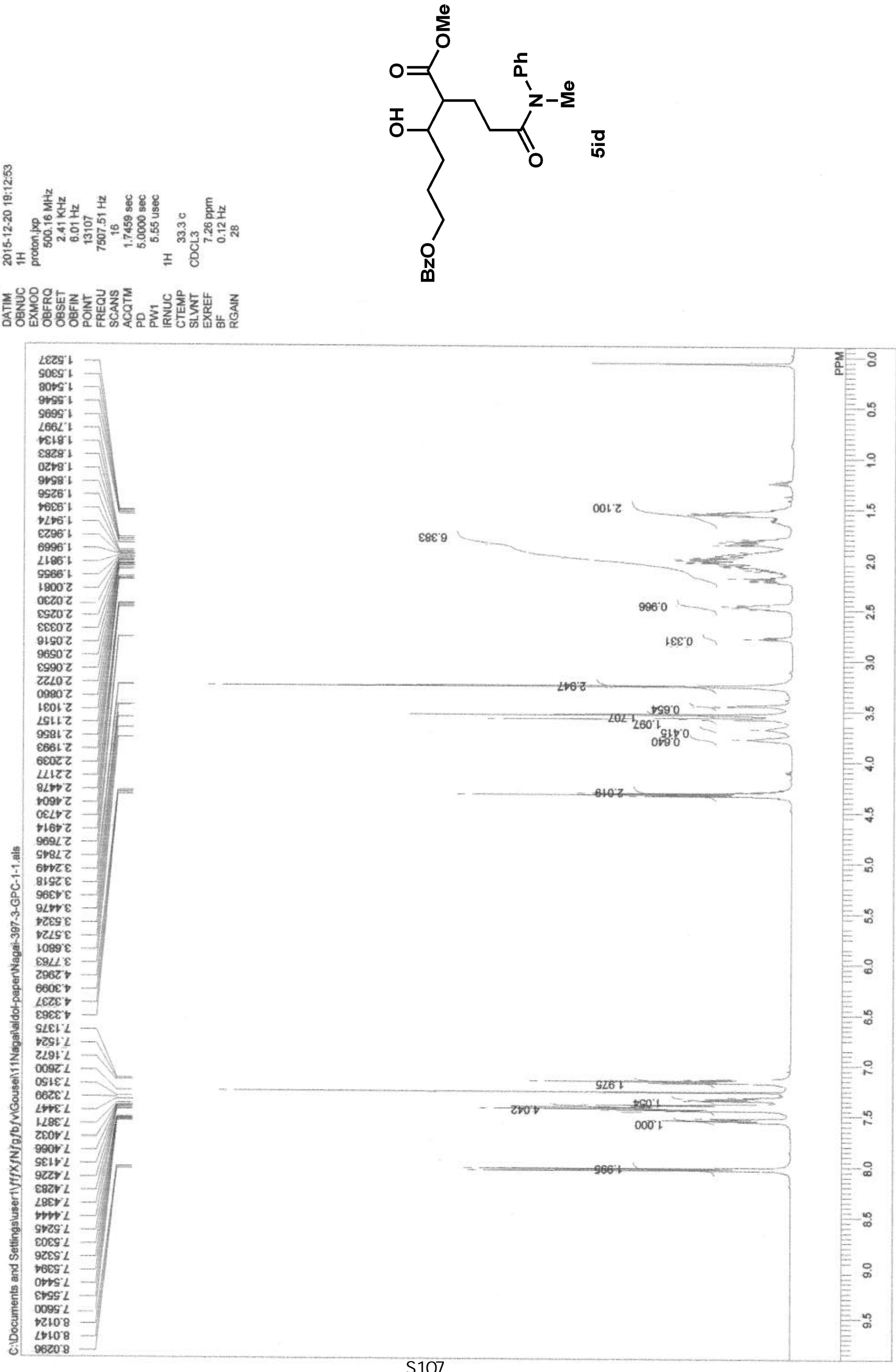

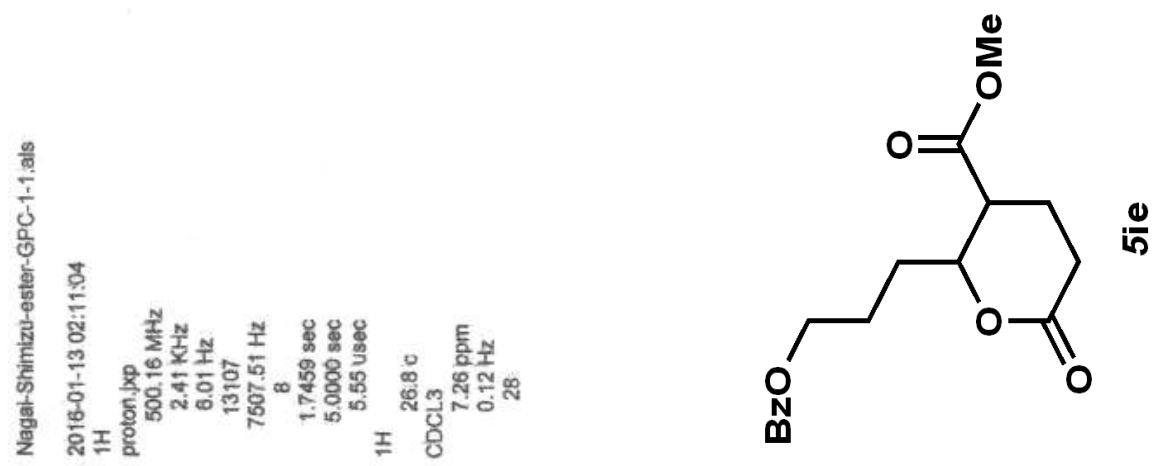

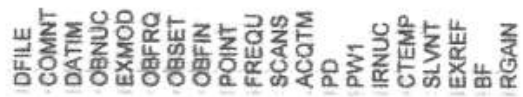

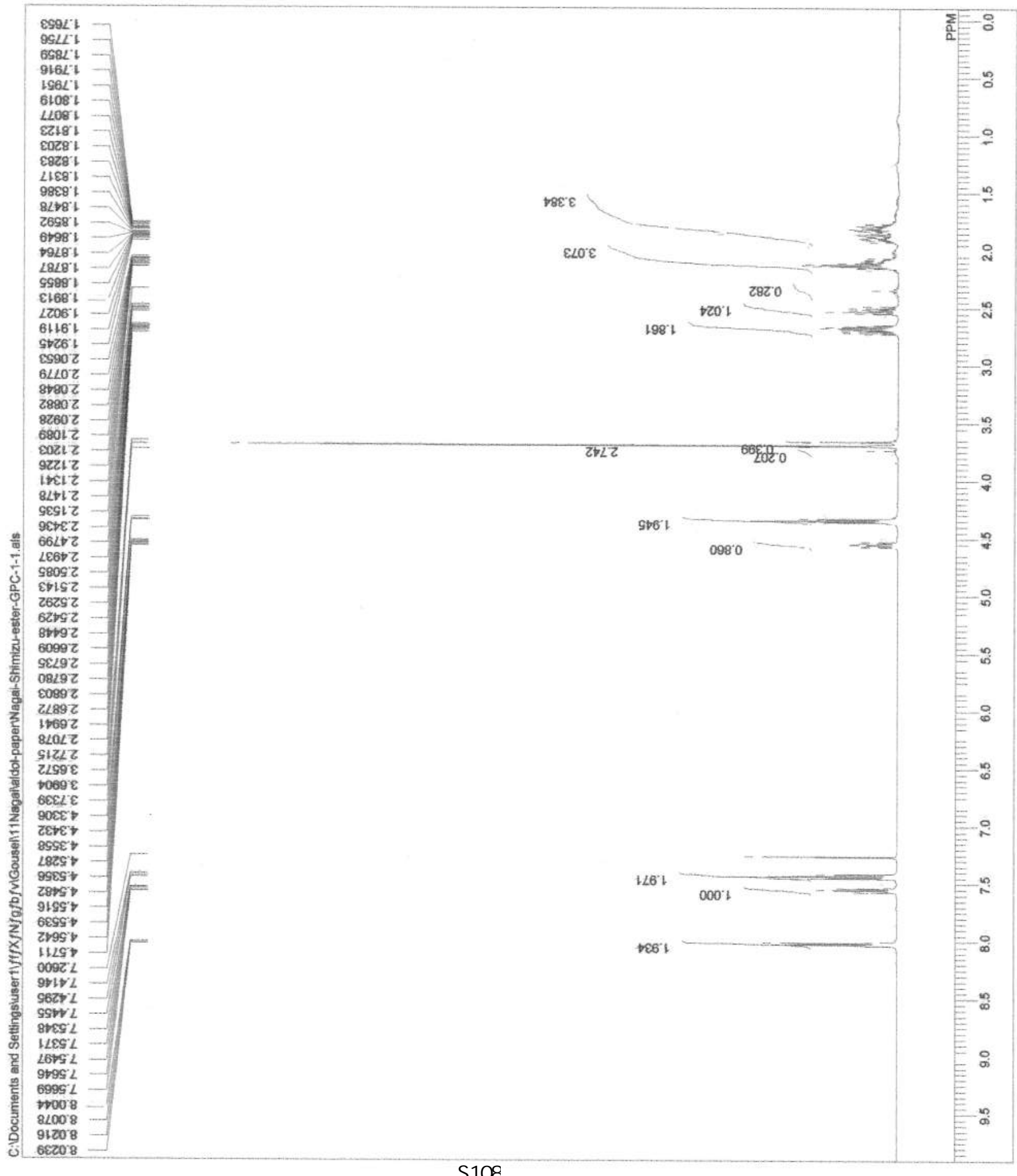



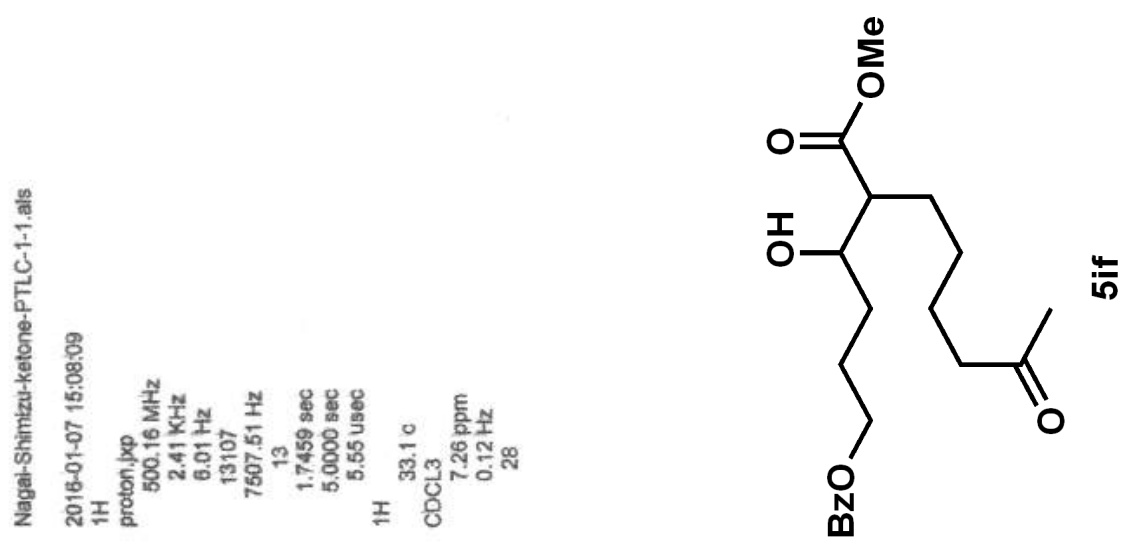

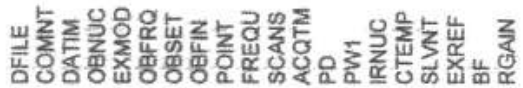

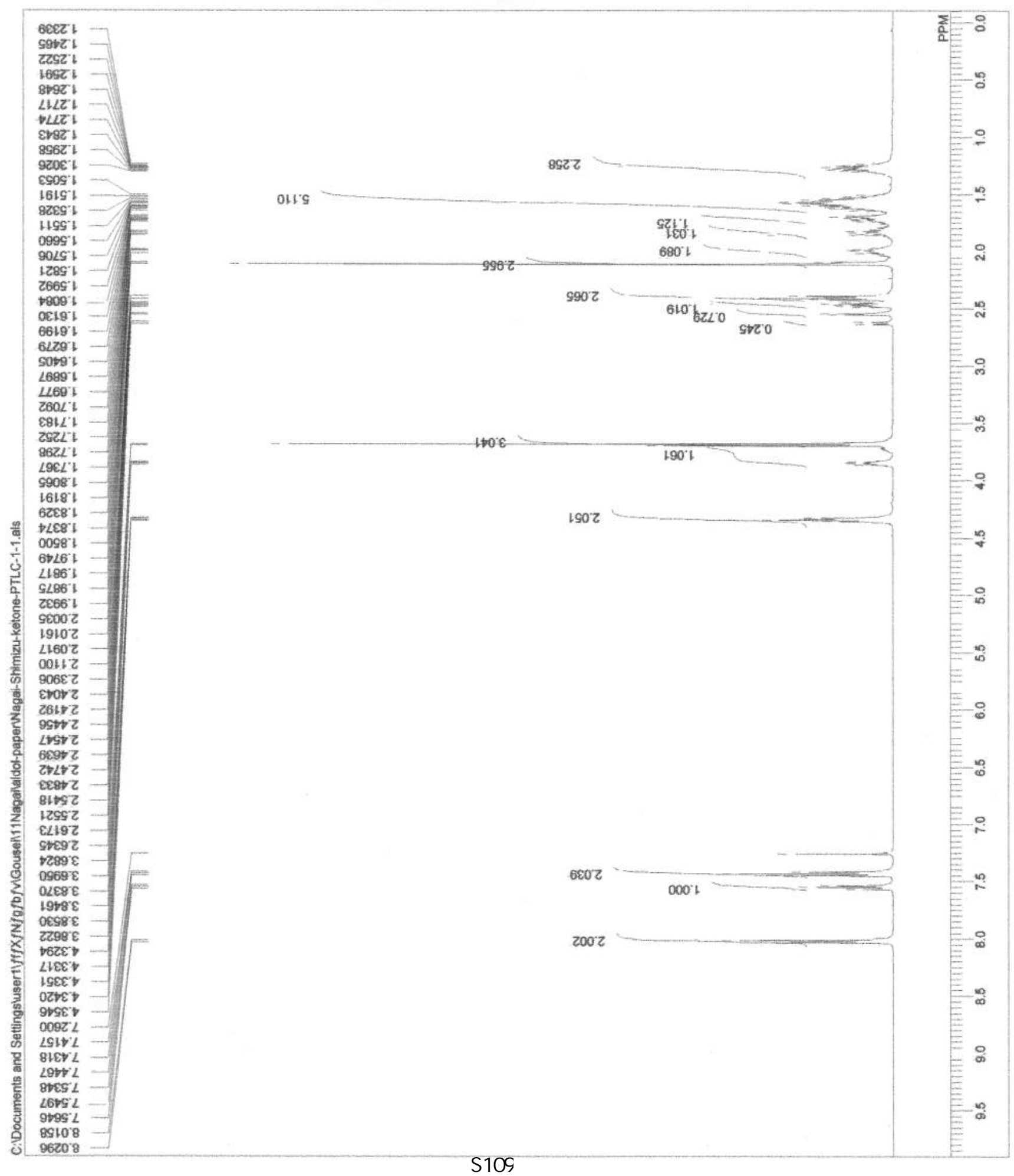



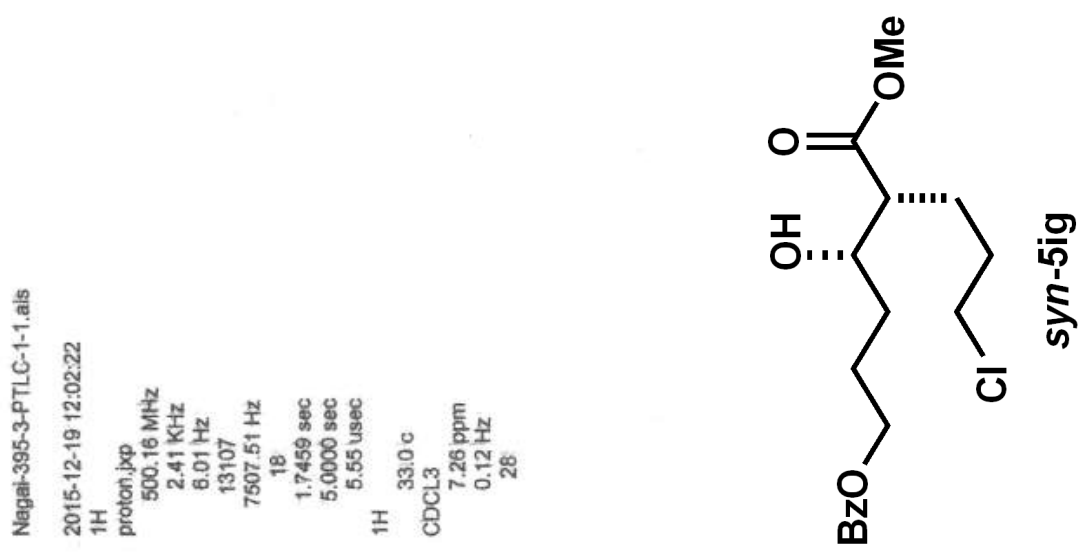

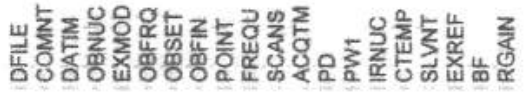

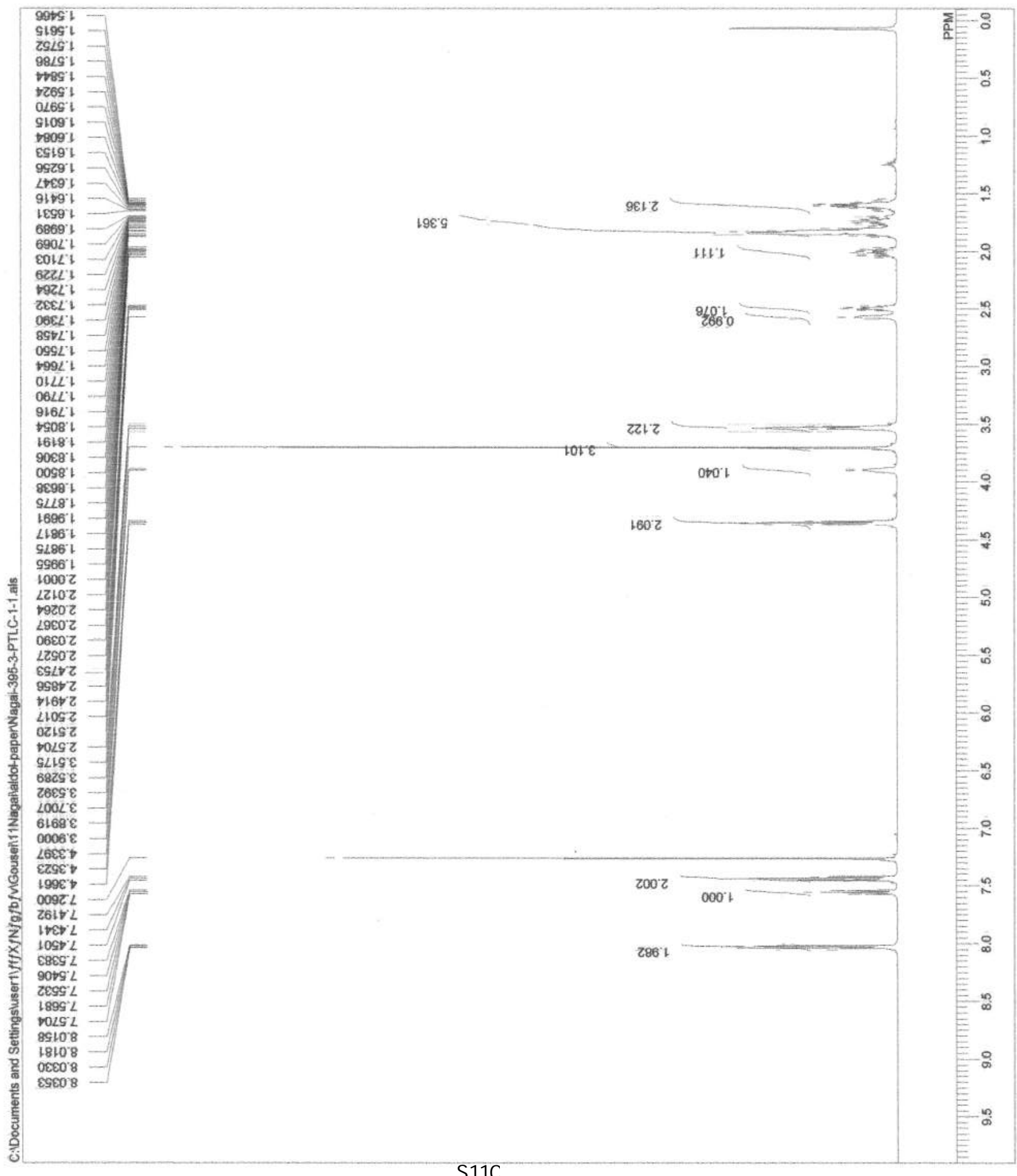




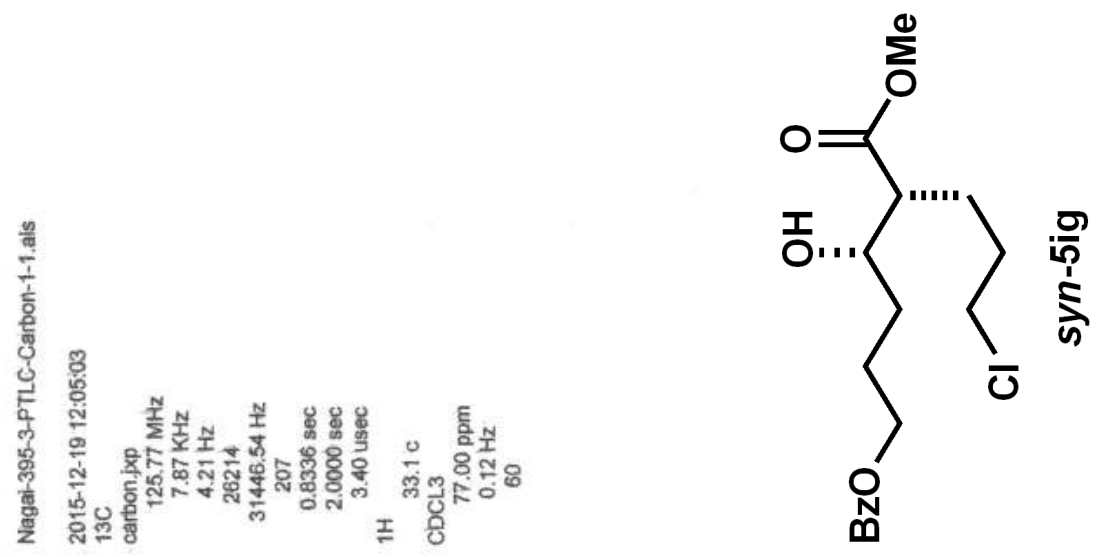

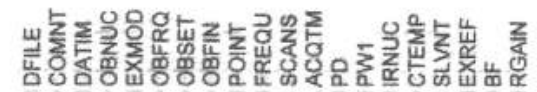

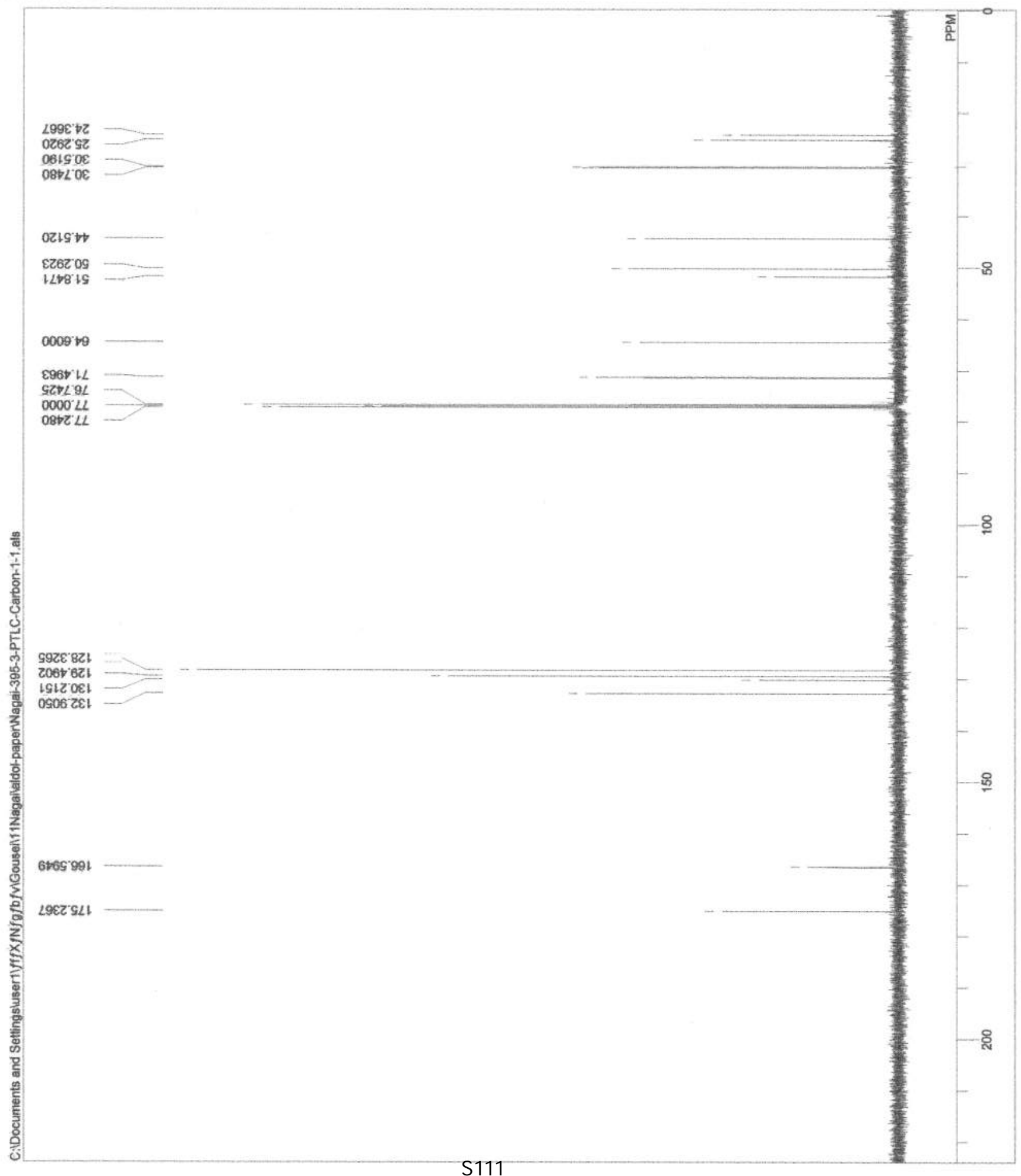



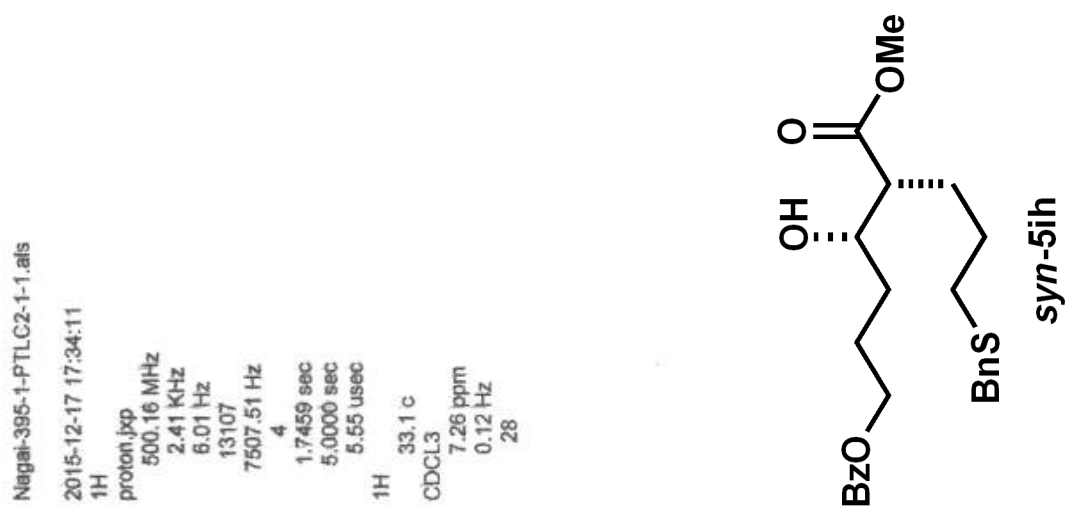

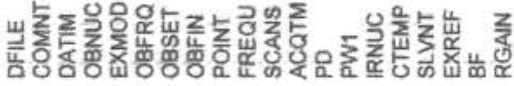

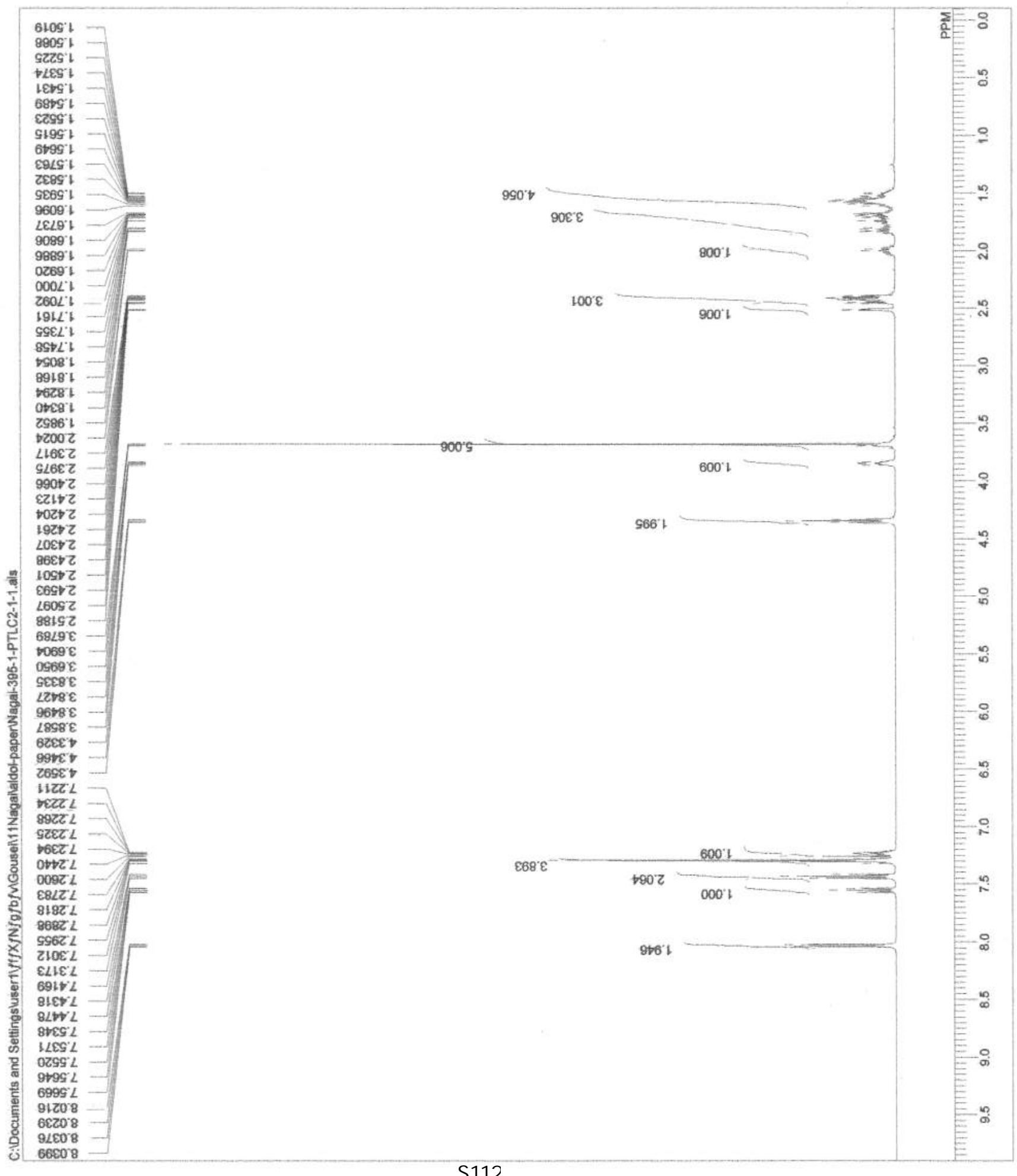



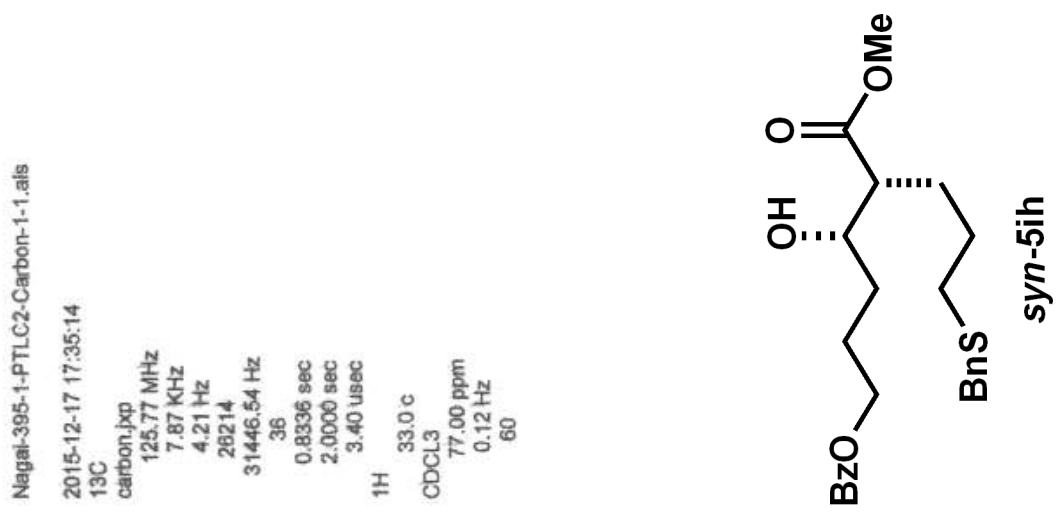

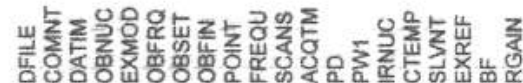

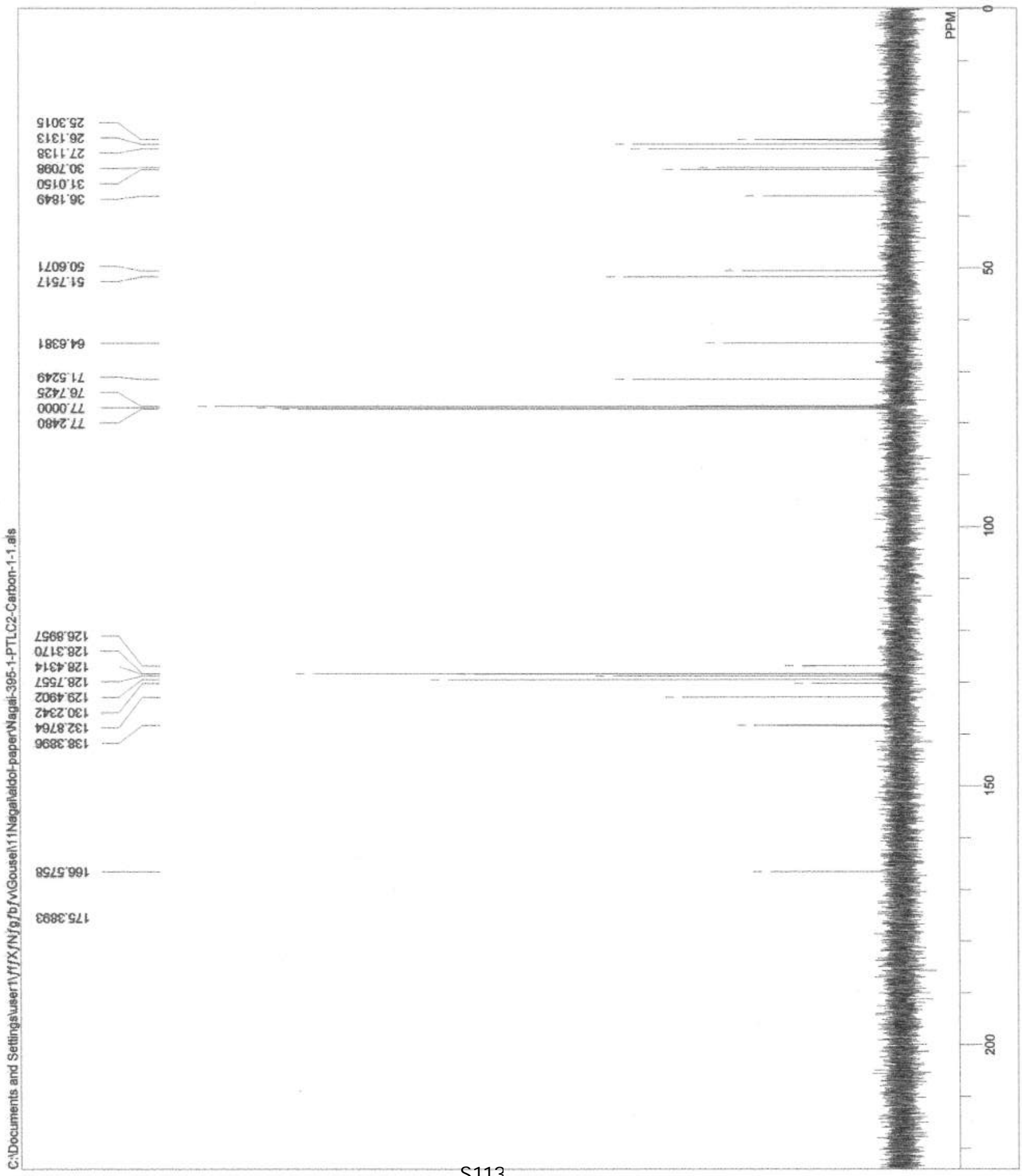



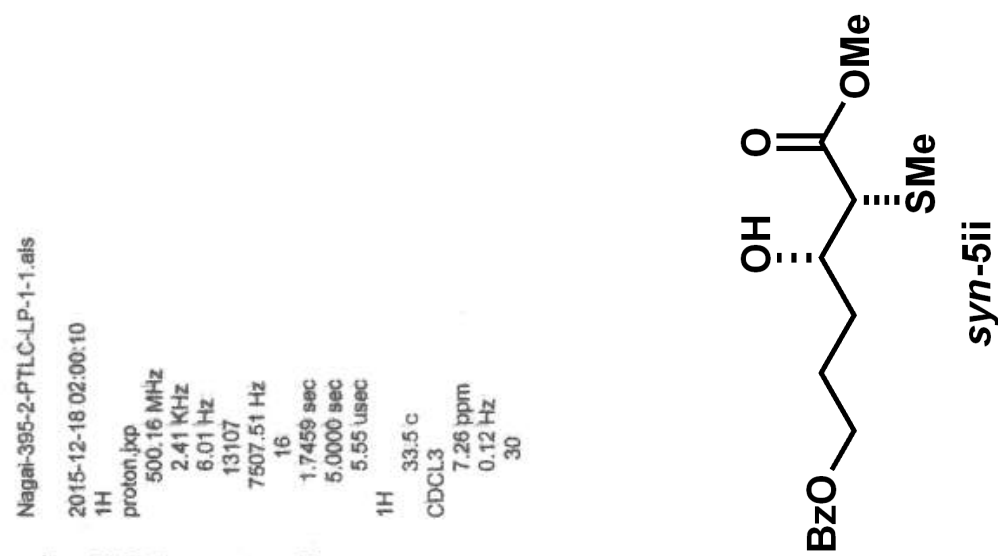

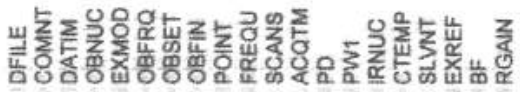

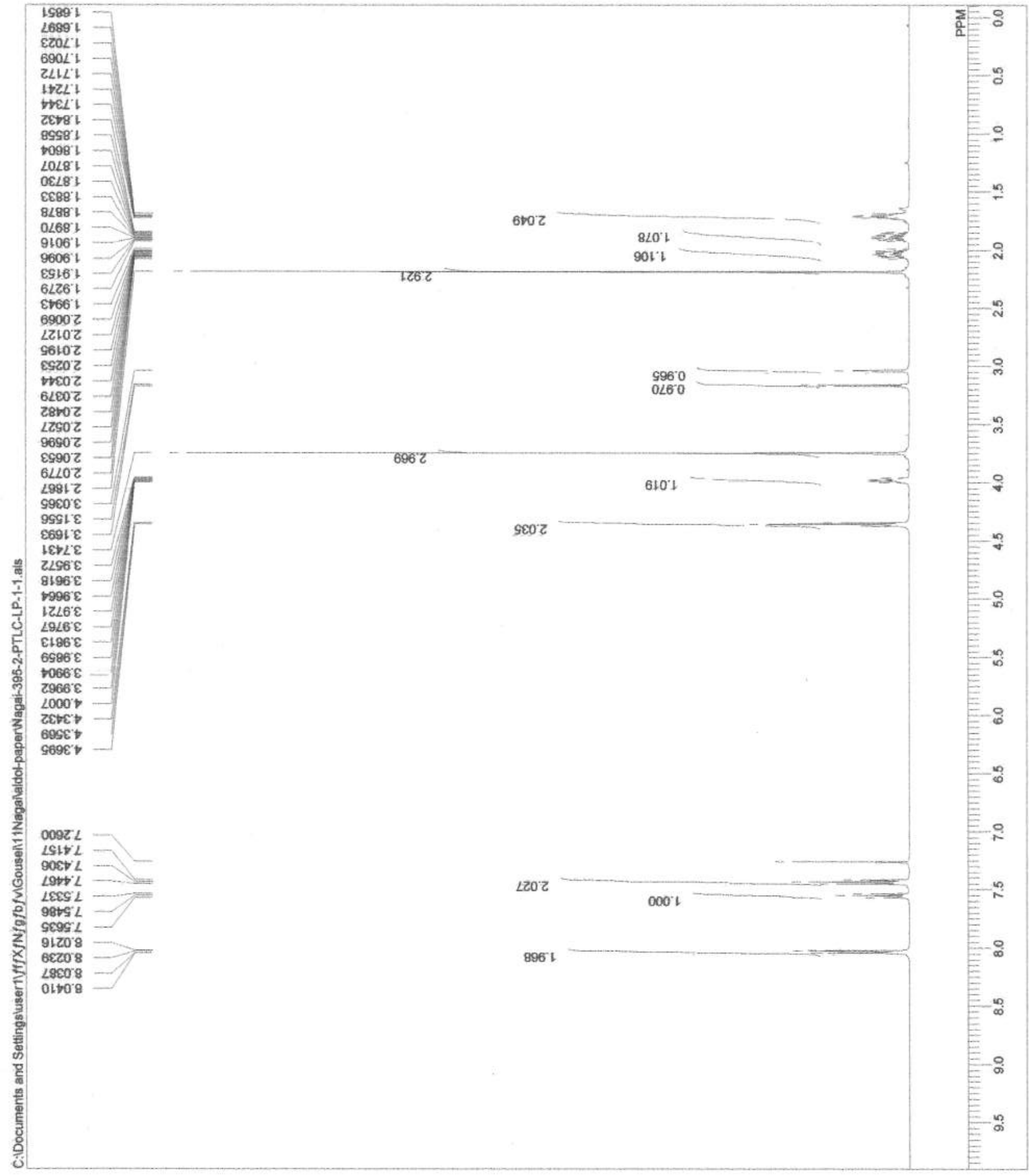



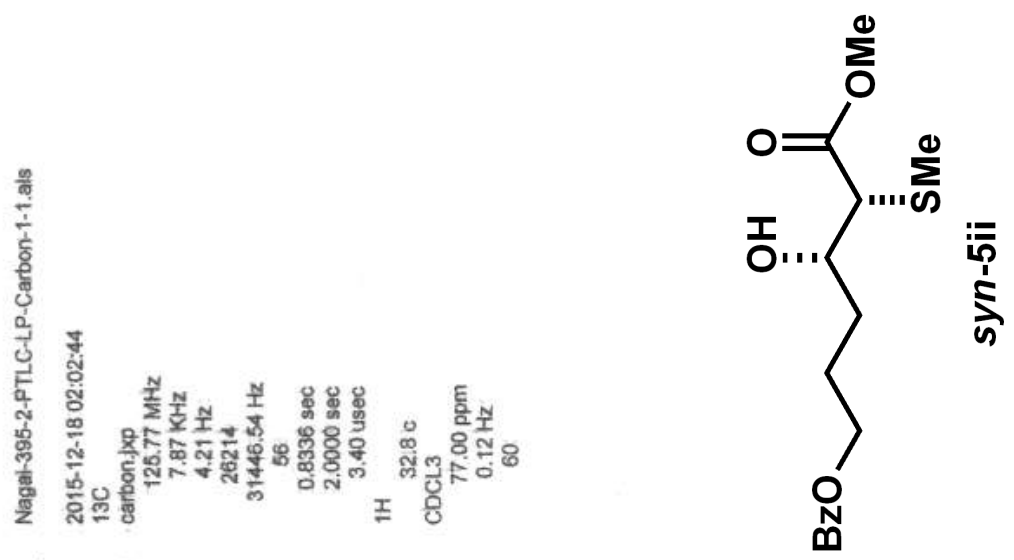

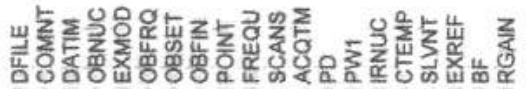

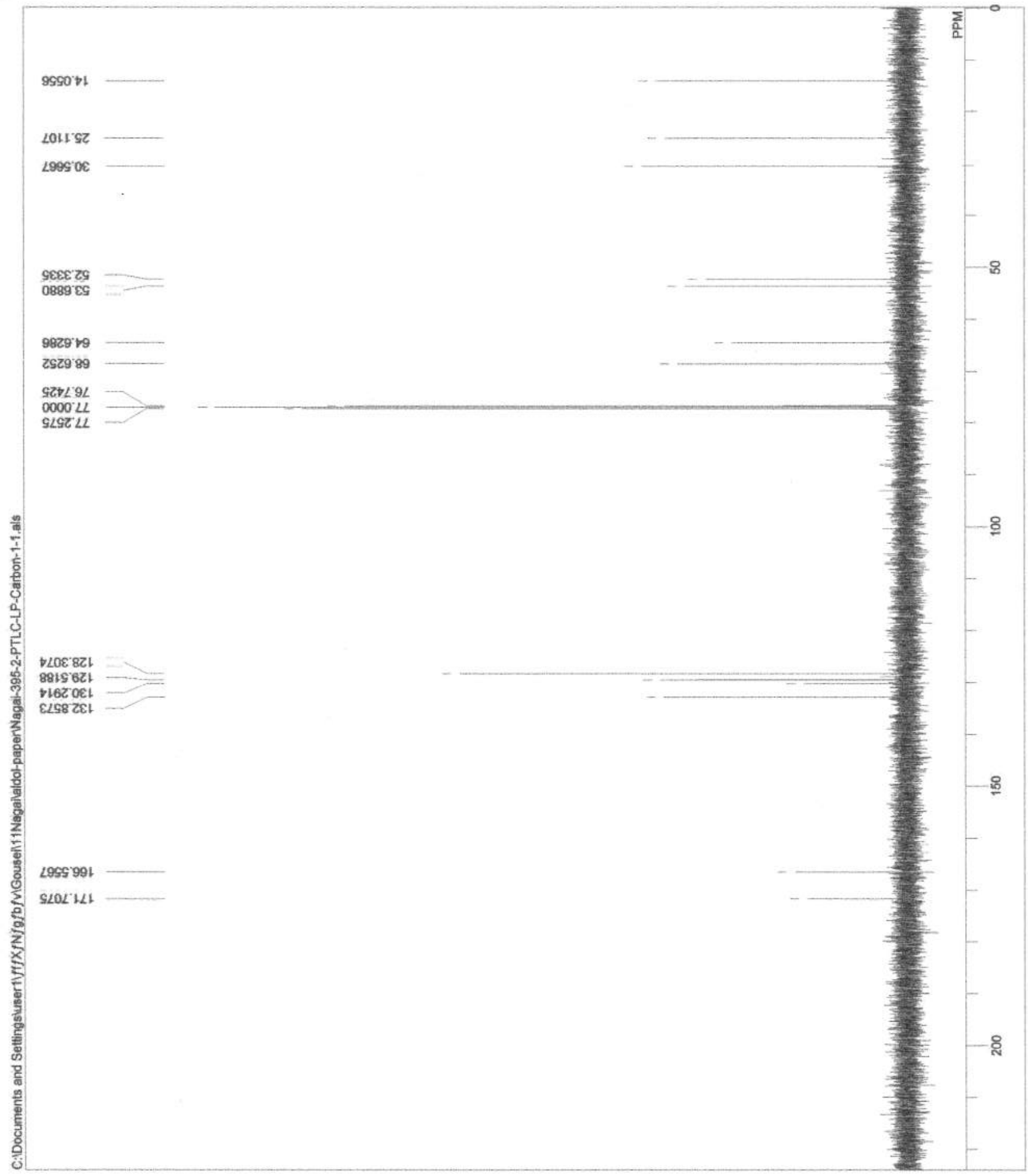



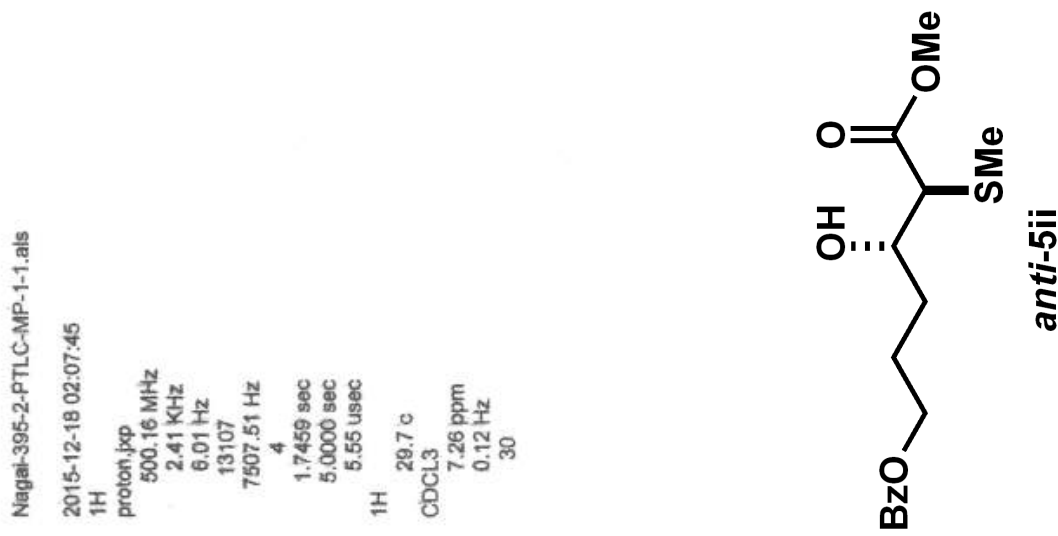

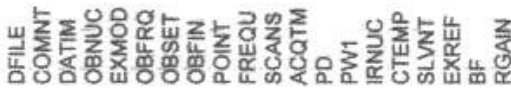

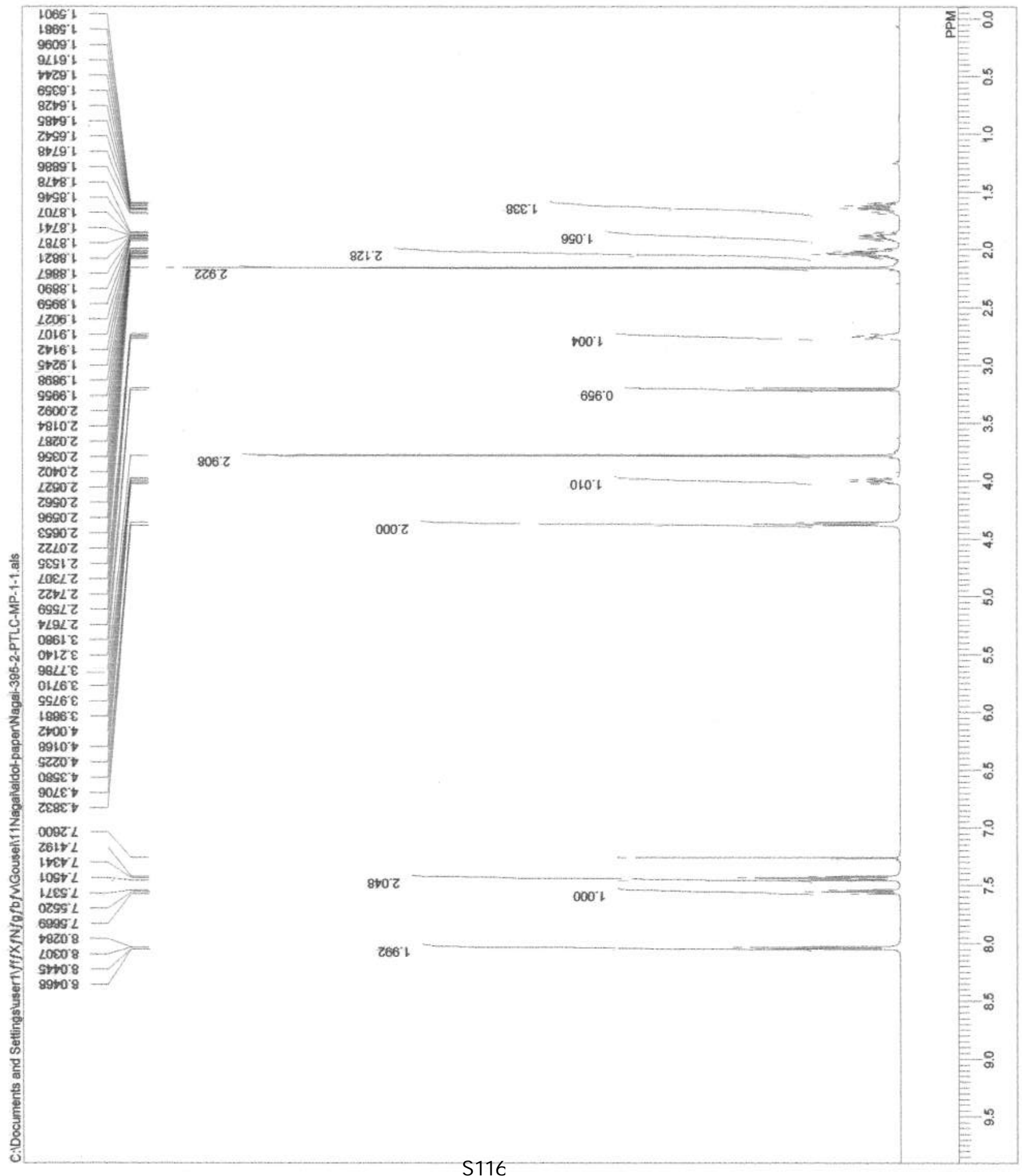



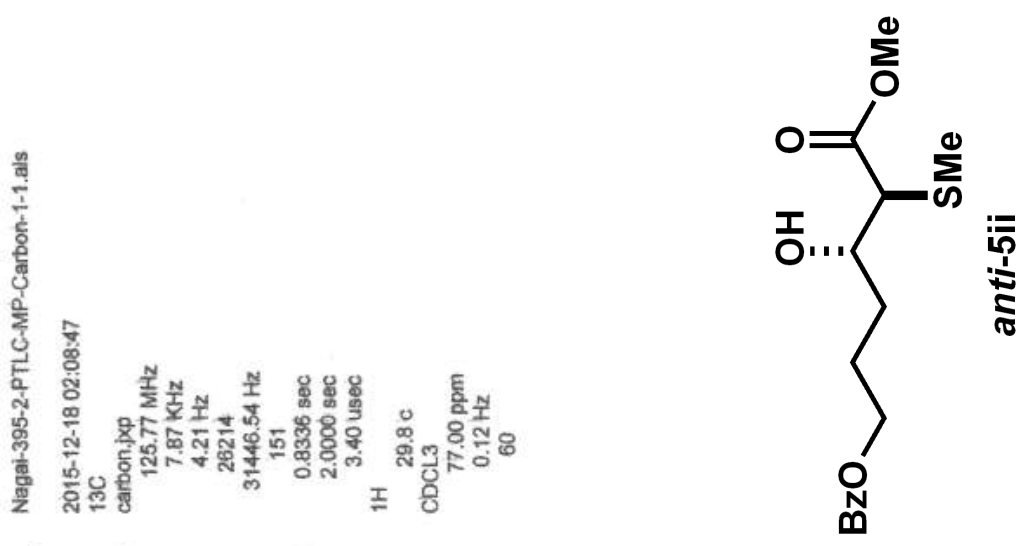

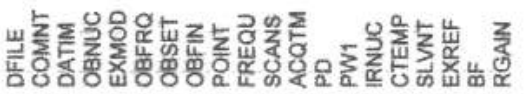

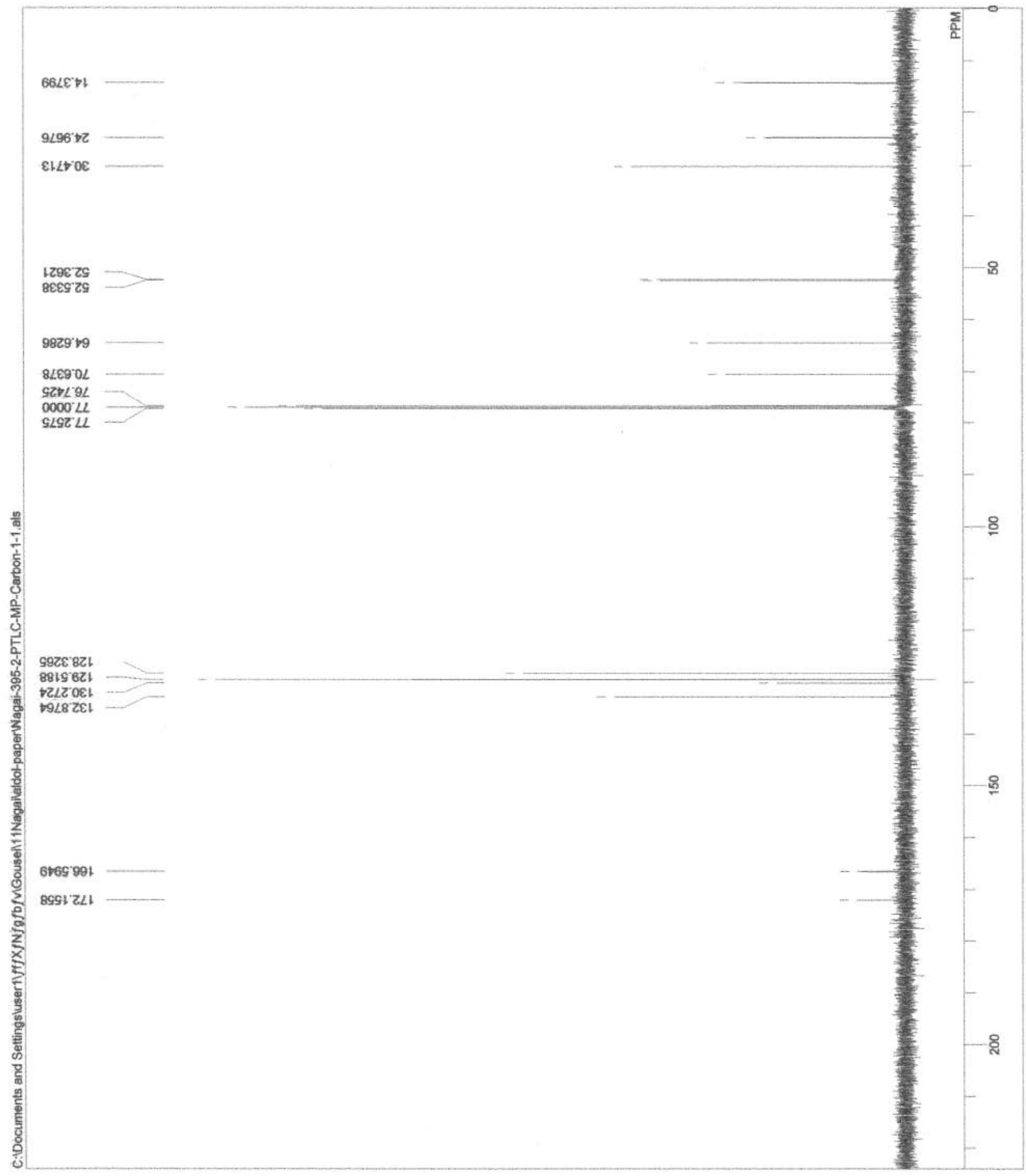




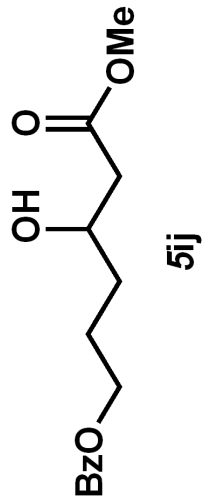

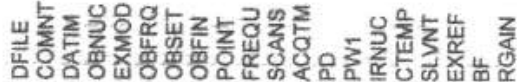

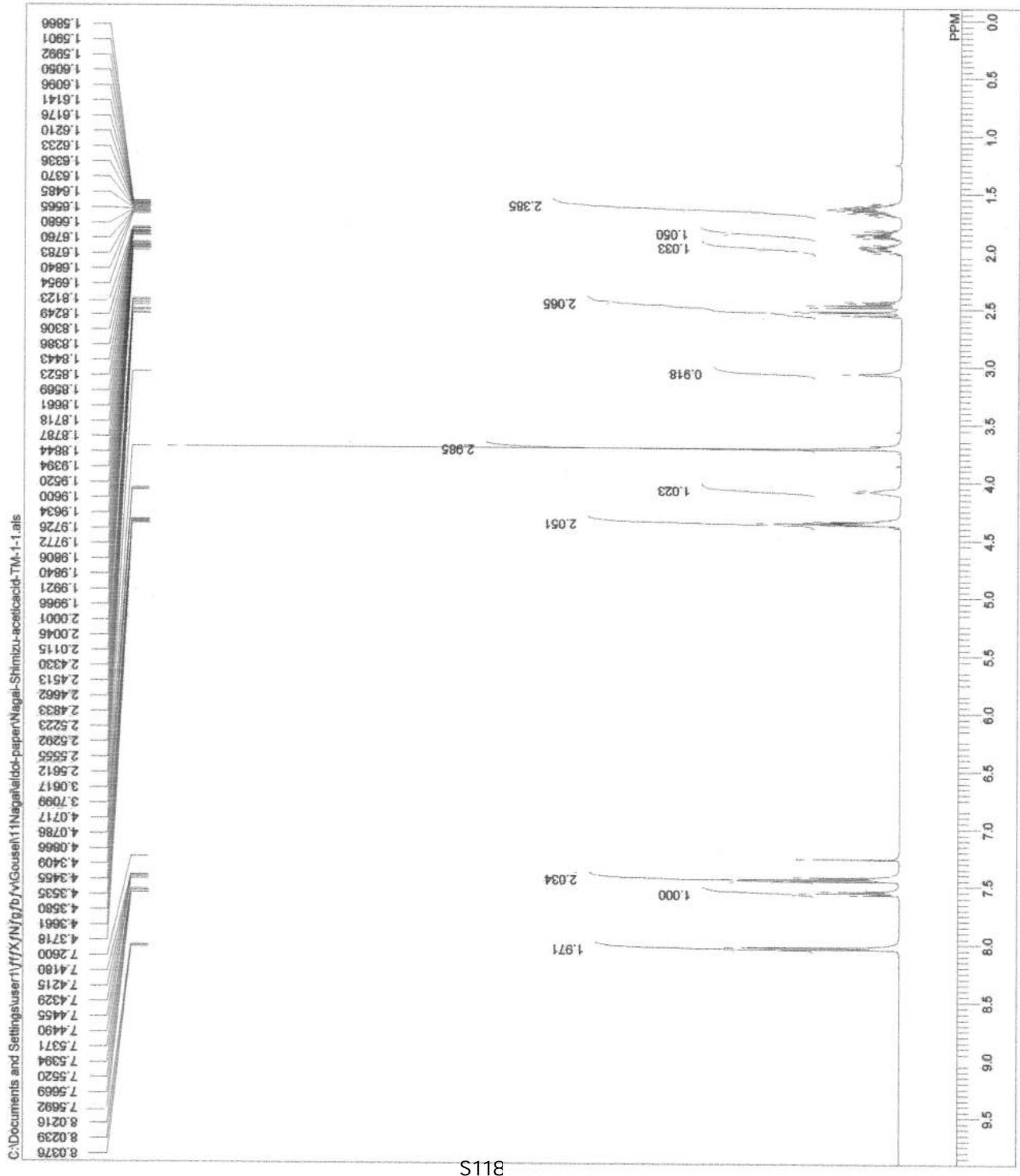



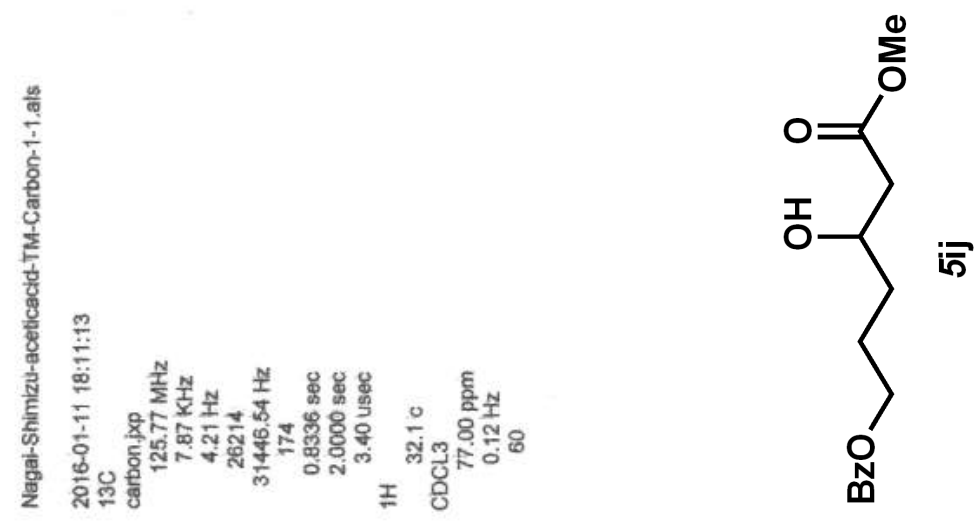

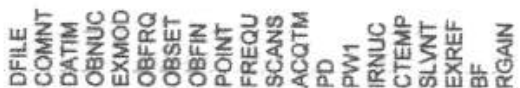

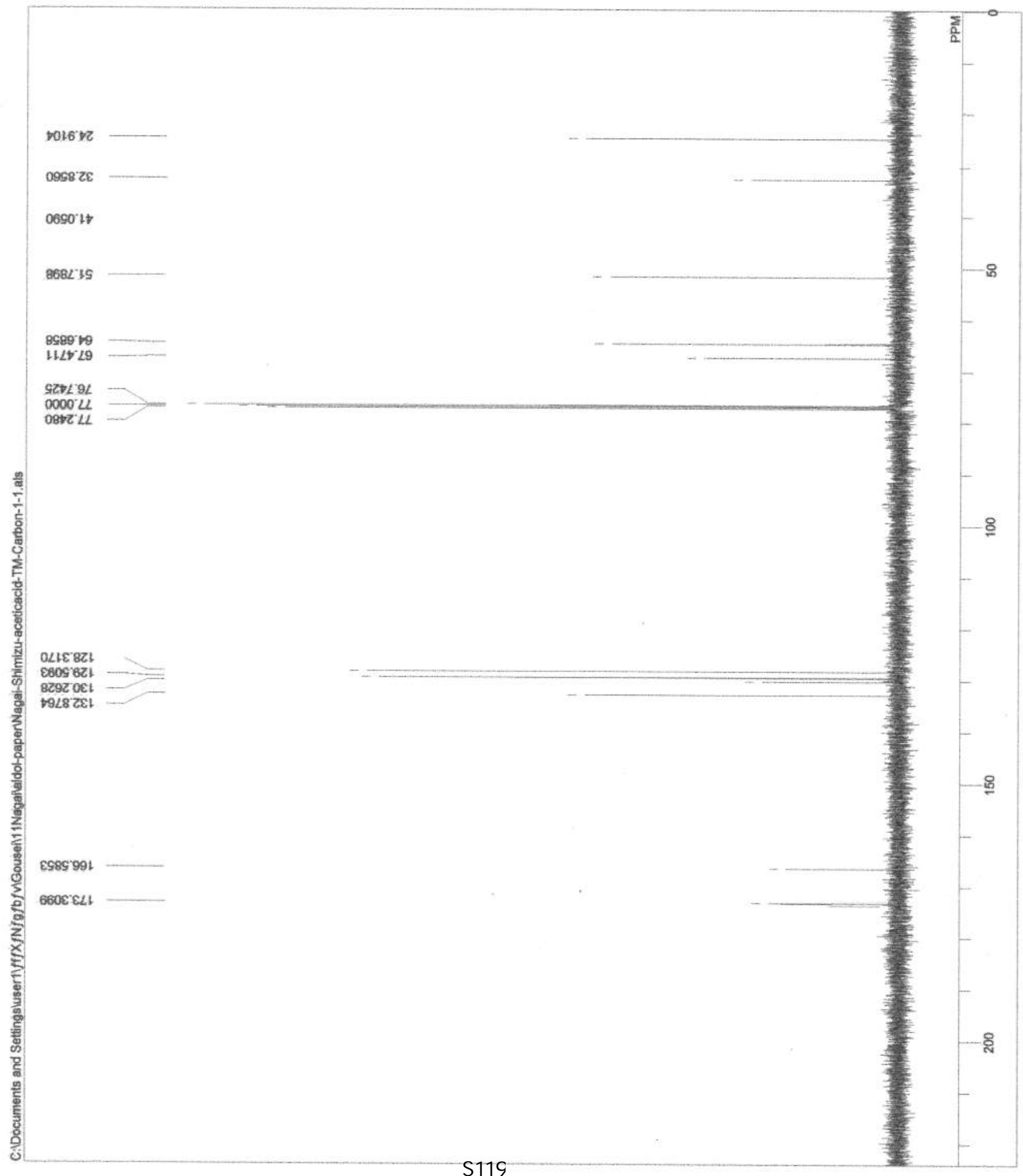




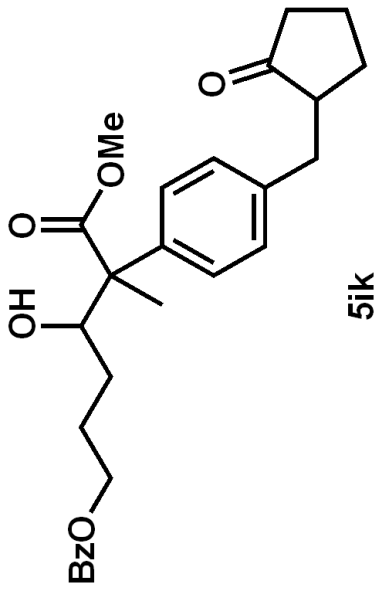

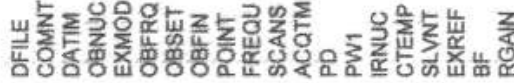

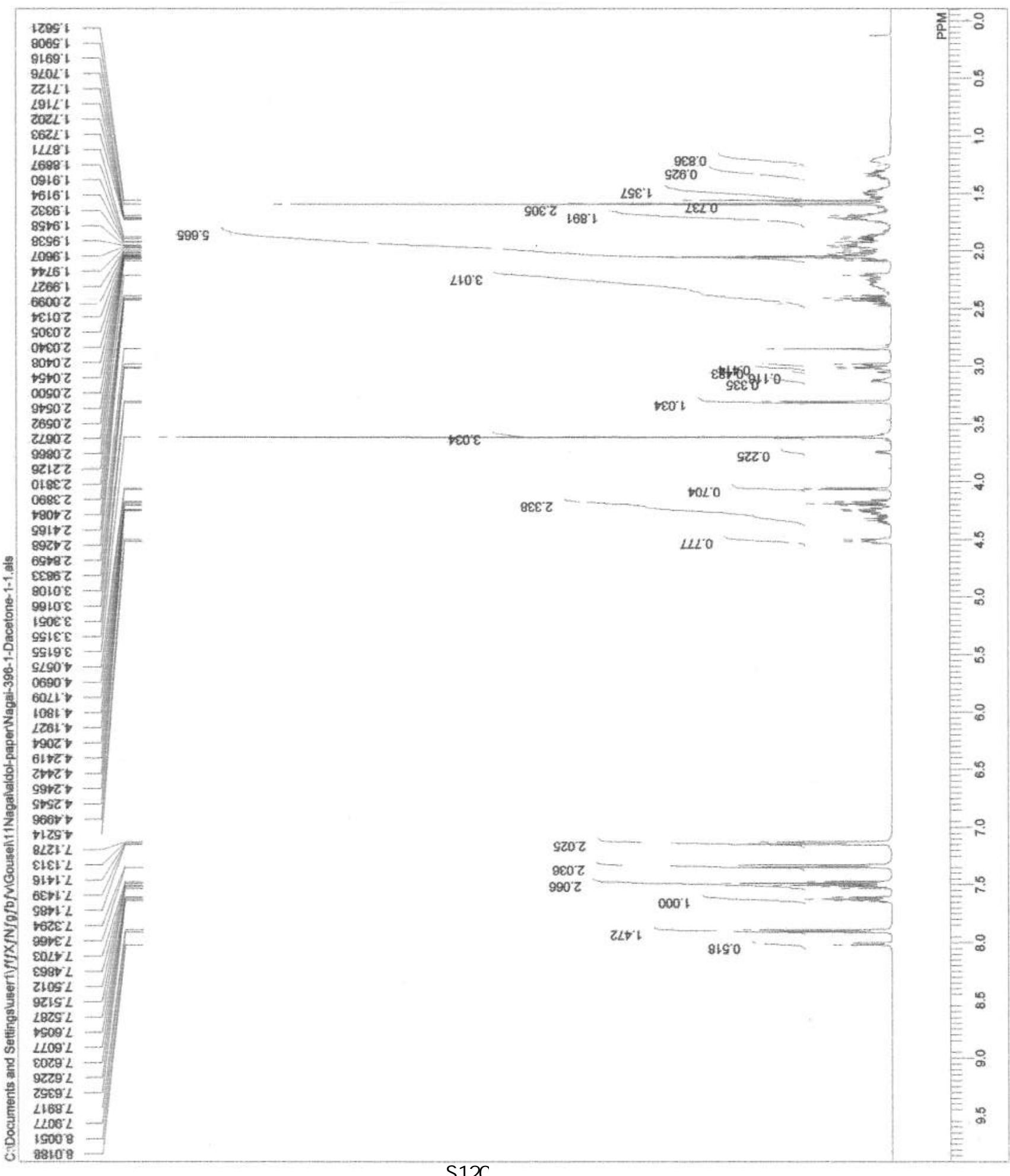




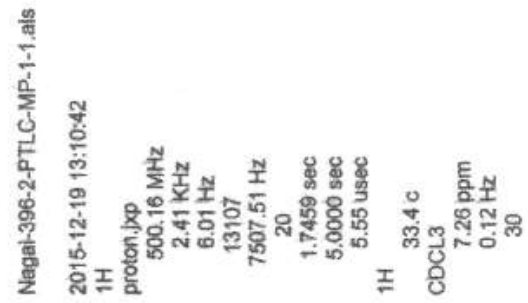

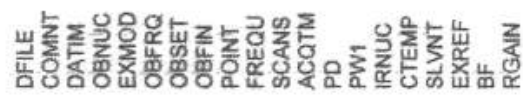
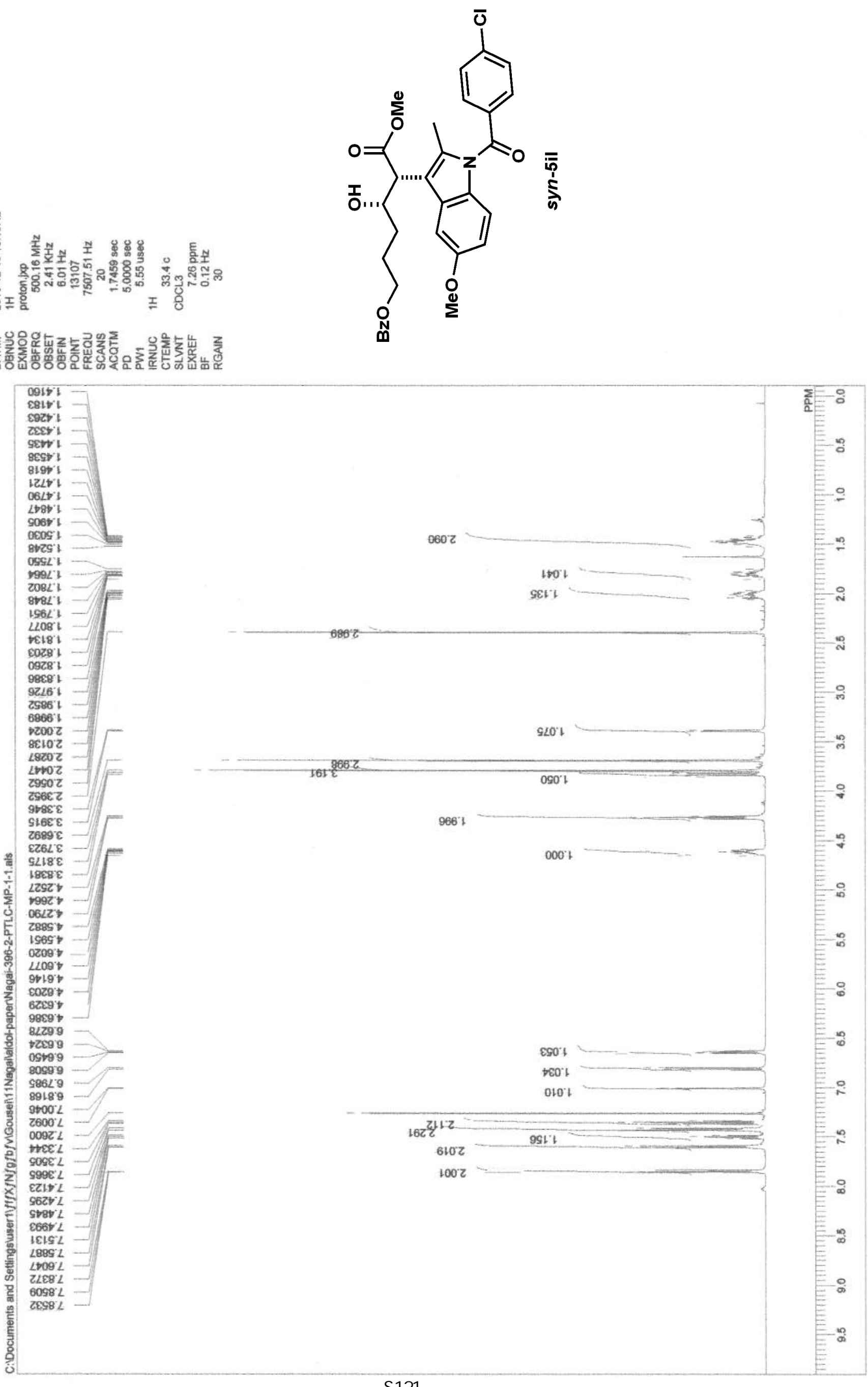


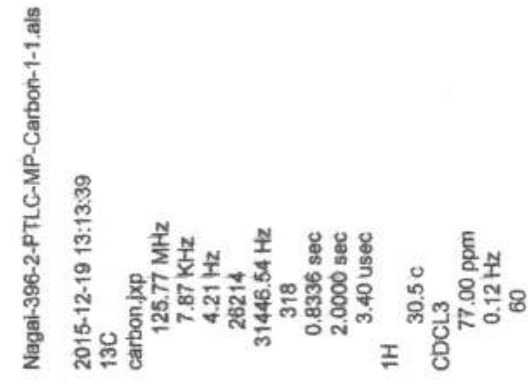

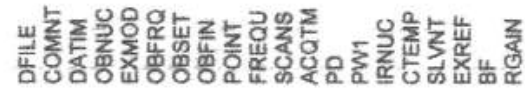
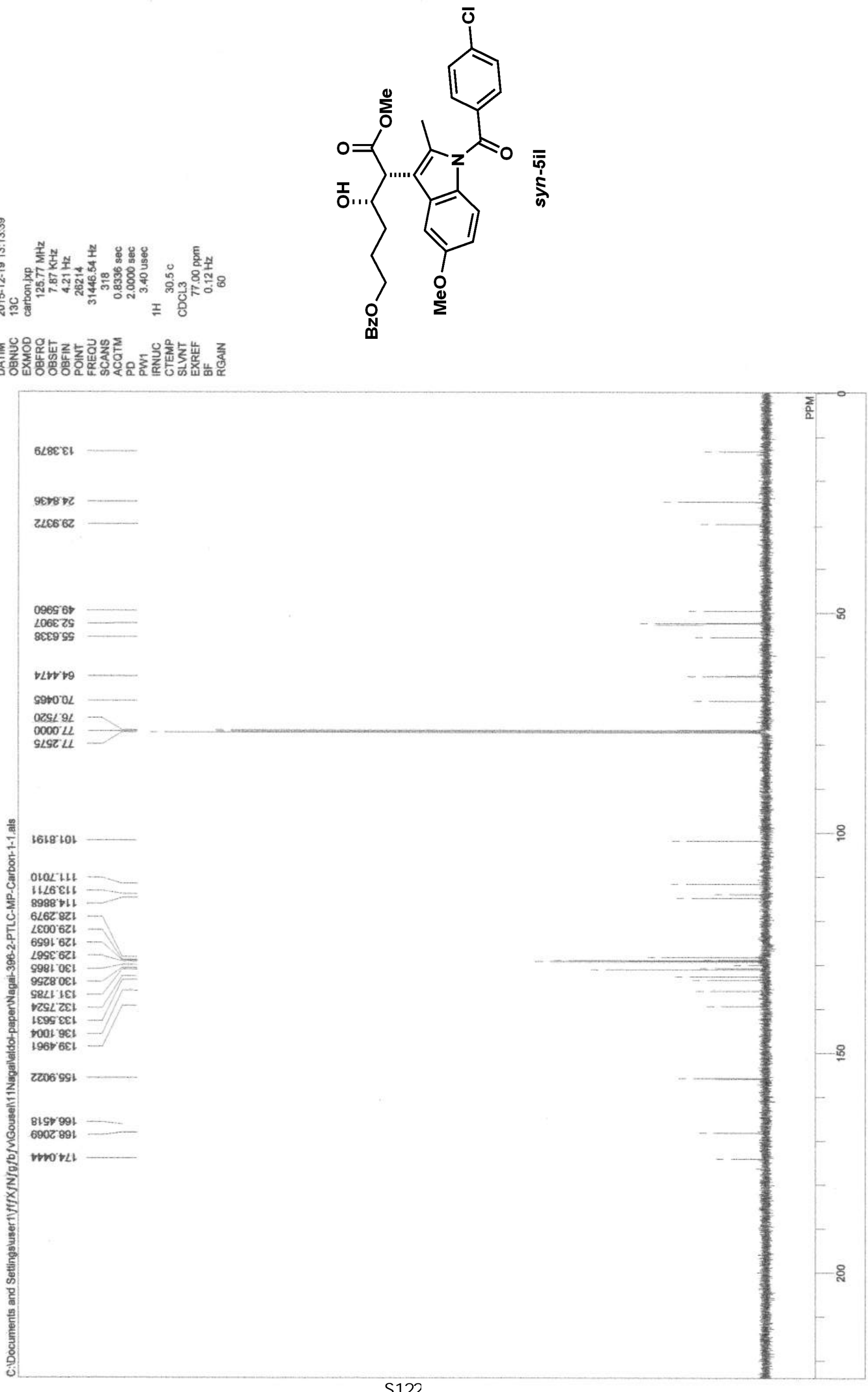


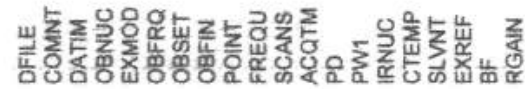
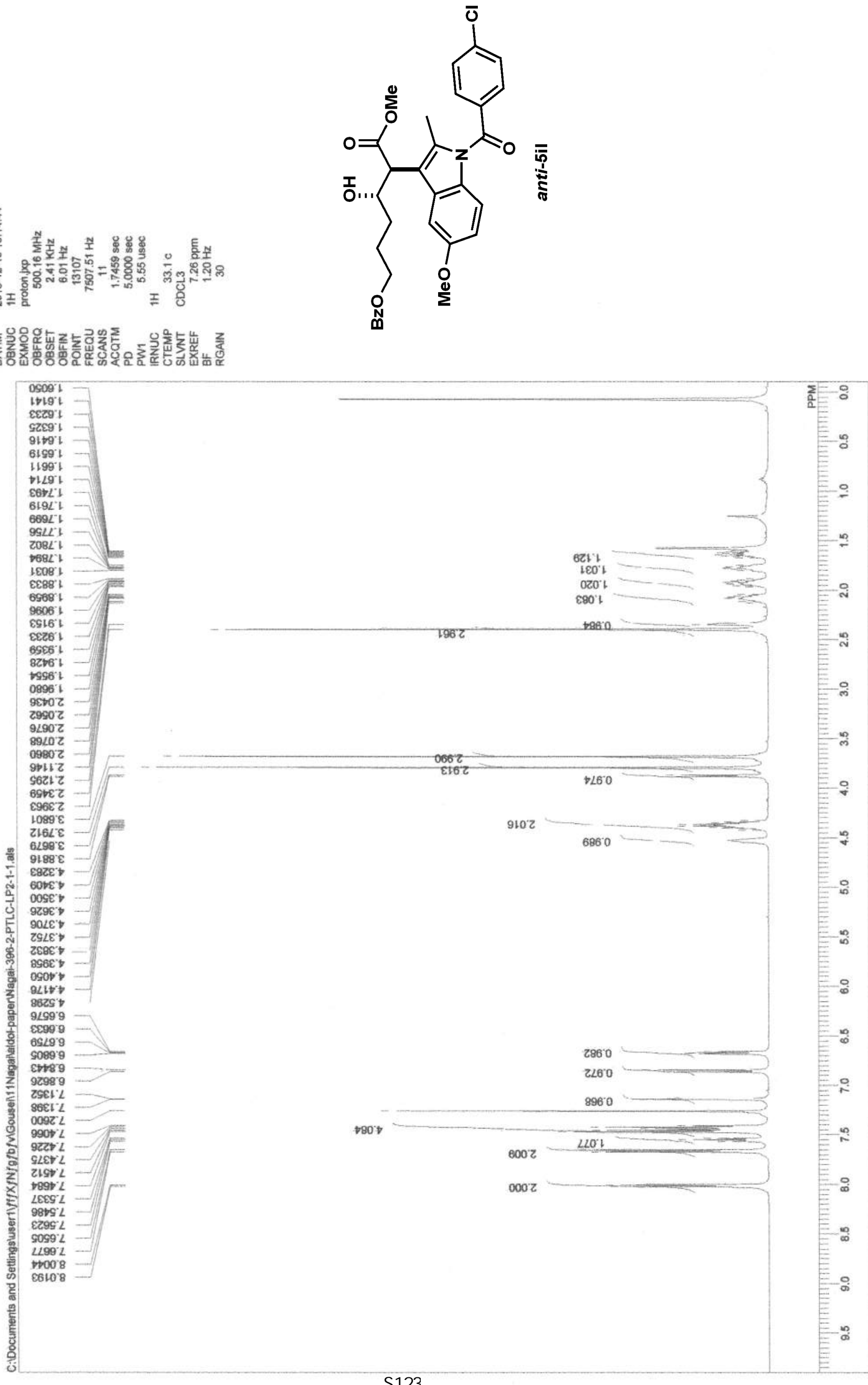


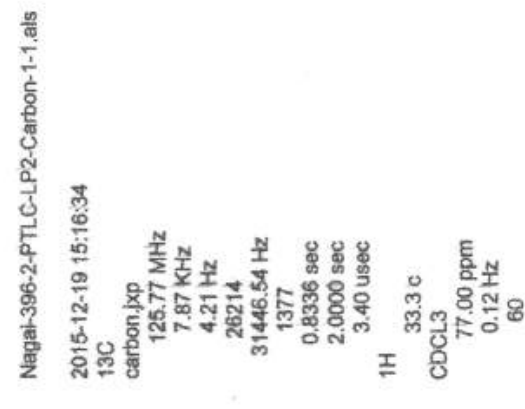

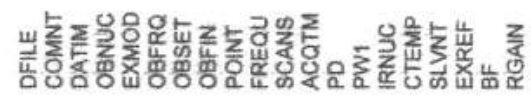
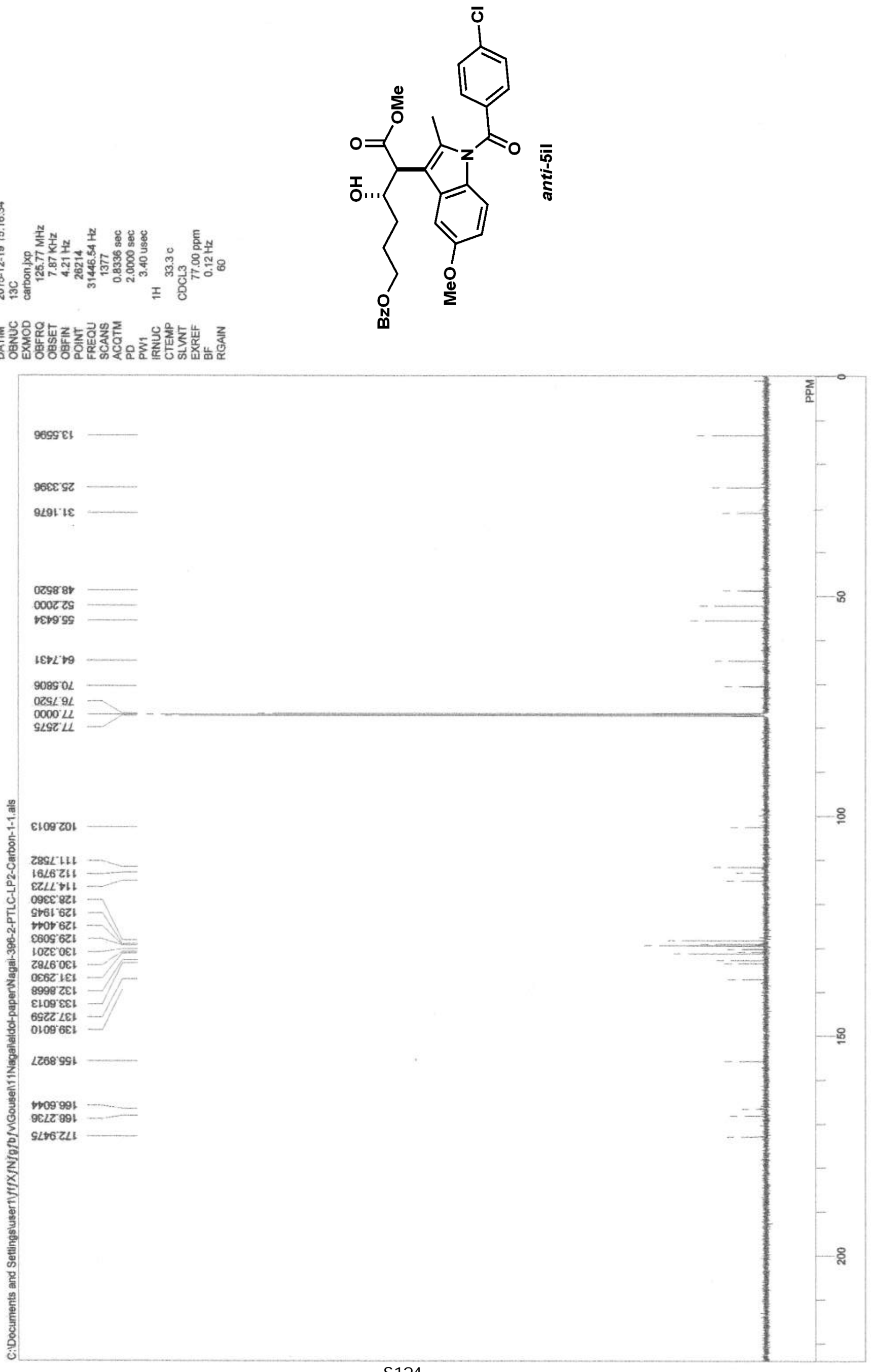


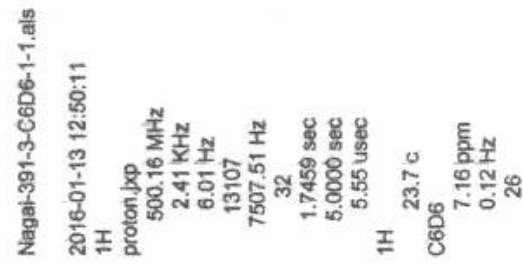

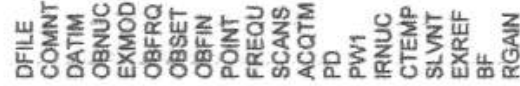
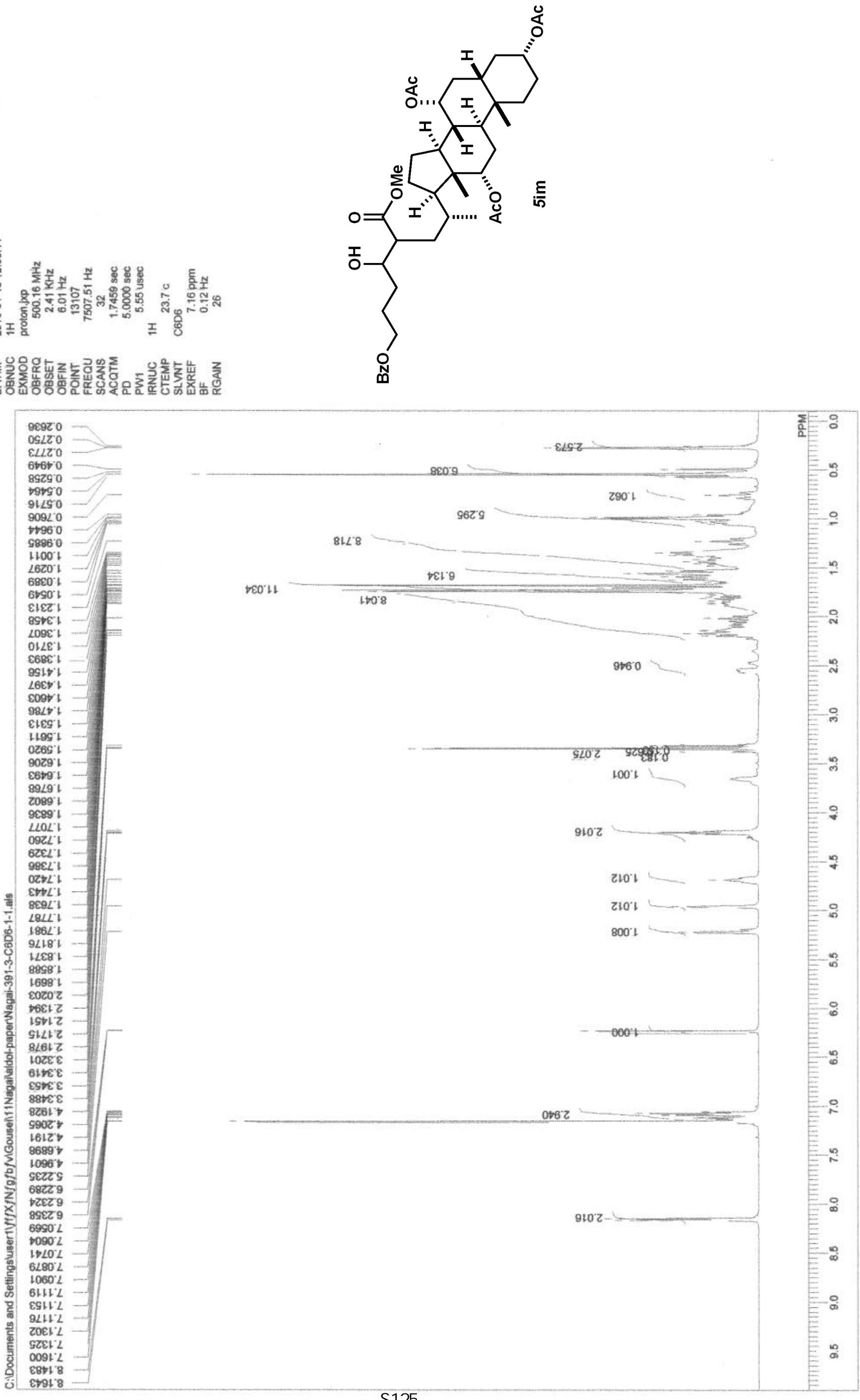


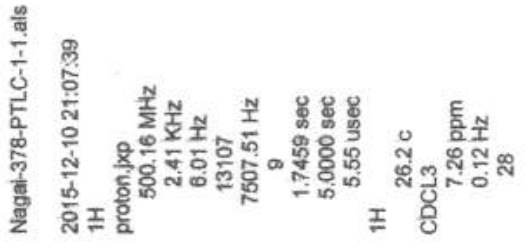

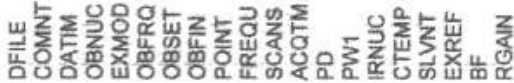
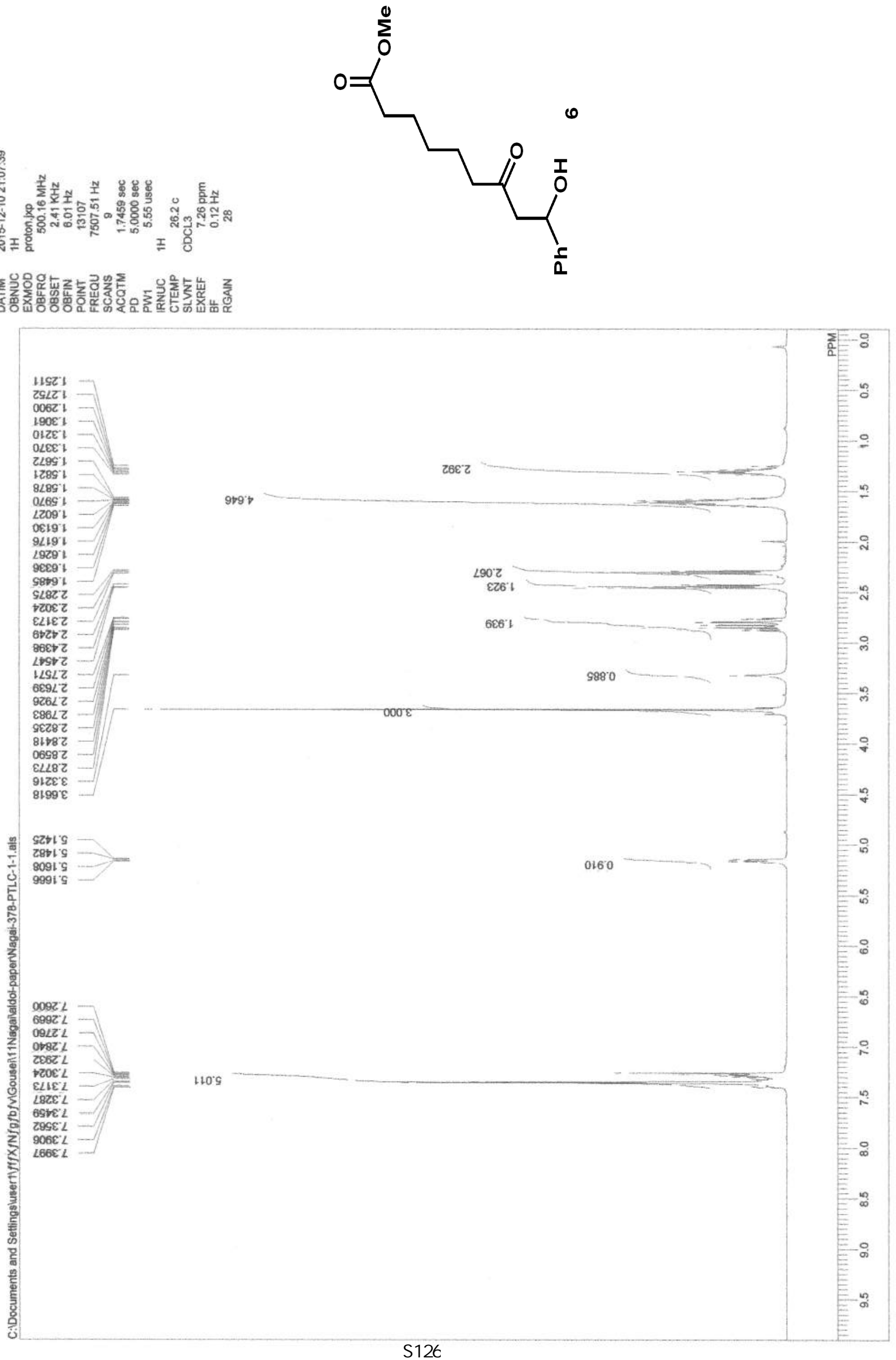

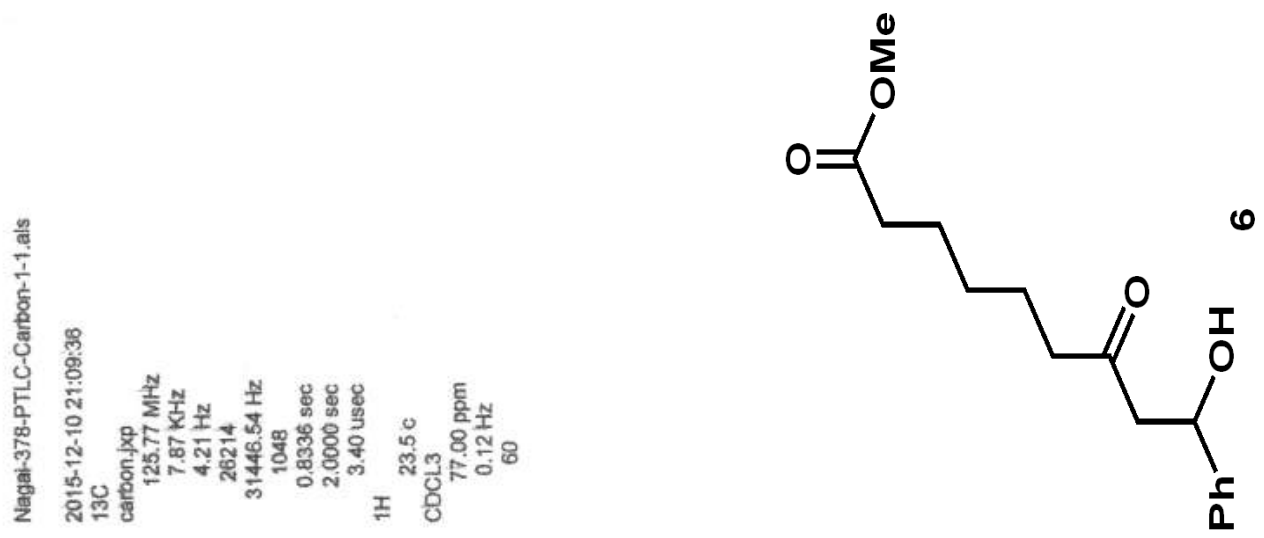

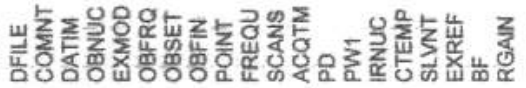

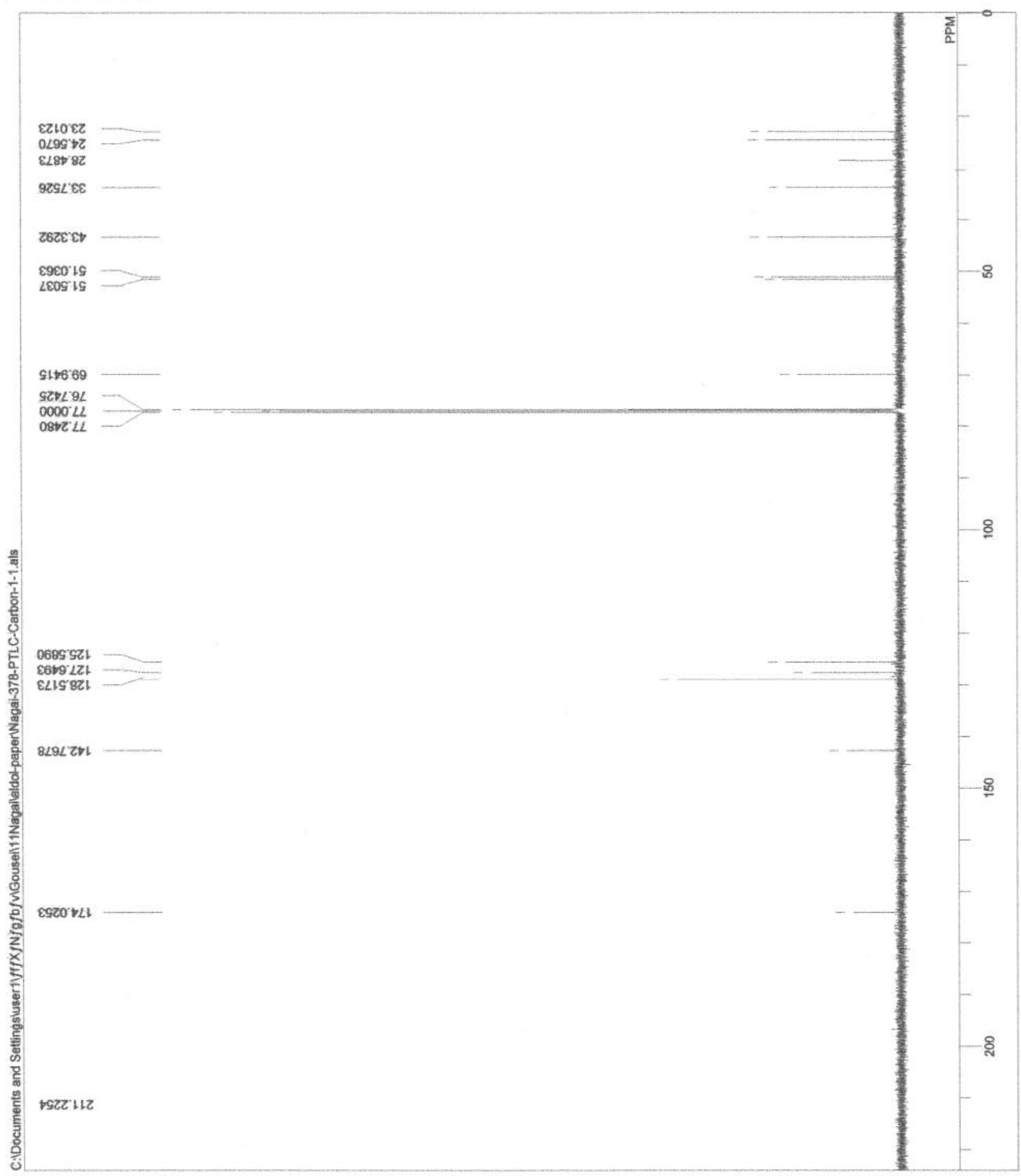

